FÁBIO HIDEKI ASHIKAWA

MITIGAÇÃO DE GOLFADAS EM SISTEMAS OFFSHORE UTILIZANDO MODELO DINÂMICO SIMPLIFICADO

São Paulo 
FÁBIO HIDEKI ASHIKAWA

\title{
MITIGAÇÃO DE GOLFADAS EM SISTEMAS OFFSHORE UTILIZANDO MODELO DINÂMICO SIMPLIFICADO
}

\author{
Dissertação apresentada à Escola \\ Politécnica da Universidade de São Paulo \\ para obtenção do título de Mestre em \\ Ciências
}

São Paulo 
FÁBIO HIDEKI ASHIKAWA

\section{MITIGAÇÃO DE GOLFADAS EM SISTEMAS OFFSHORE UTILIZANDO MODELO DINÂMICO SIMPLIFICADO}

Dissertação apresentada à Escola Politécnica da Universidade de São Paulo para obtenção do título de Mestre em Ciências

Área de Concentração: Engenharia Química

Orientador: Prof. Dr. Song Won Park 
Este exemplar foi revisado e corrigido em relação à versão original, sob responsabilidade única do autor e com a anuência de seu orientador.

São Paulo de de

Assinatura do autor:

Assinatura do orientador:

Catalogação-na-publicação

ASHIKAWA, FABIO HIDEKI

MITIGAÇÃO DE GOLFADAS EM SISTEMAS OFFSHORE UTILIZANDO MODELO DINÂMICO SIMPLIFICADO / F. H. ASHIKAWA -- versão corr. -- São Paulo, 2017. $127 \mathrm{p}$.

Dissertação (Mestrado) - Escola Politécnica da Universidade de São Paulo. Departamento de Engenharia Química.

1.Riser slug advanced control 2.oil and gas 3.offshore production 4.choke valve 5.slugging flow models I.Universidade de São Paulo. Escola Politécnica. Departamento de Engenharia Química II.t. 


\section{AGRADECIMENTOS}

Ao meu orientador, Prof. Dr. Song Won Park, pelo apoio, grande paciência e ajuda durante a elaboração desta dissertação.

Aos membros da banca de qualificação, pelas sugestões de melhoria e correções.

Aos meus pais, pela minha formação e por sempre me apoiarem.

A Petrobras, empresa na qual faço parte, pelo apoio e compreensão durante a realização deste trabalho.

A Deus, por me dar força para superar todas as dificuldades desta jornada.

Ao Departamento de Engenharia Química, pela oportunidade de realização do mestrado. 
"A mente que se abre a uma nova ideia jamais voltará ao seu tamanho original."

Albert Einstein 


\section{RESUMO}

ASHIKAWA, F.H. Mitigação de Golfadas em Sistemas Offshore utilizando Modelo Dinâmico Simplificado. São Paulo, 2017. Dissertação (Mestrado em Ciências - Engenharia Química) - Escola Politécnica da Universidade de São Paulo, 2017.

Atualmente, há inúmeras instalações offshore operando em regimes instáveis de escoamento. Tal regime é resultado de diâmetros superdimensionados das linhas de produção ou baixa vazão dos poços. Devese notar que, muitas vezes, o regime intermitente se instala no final da vida produtiva dos poços, devido ao declínio natural dos reservatórios, o que causa uma redução da produção do campo de petróleo. Tal instabilidade traz dificuldades na produção offshore, pois pode causar danos nos equipamentos da plataforma ou perdas de produção. Neste âmbito, é necessário estudar meios de atenuar ou suprimir este tipo de regime de escoamento. $O$ objetivo deste trabalho é desenvolver métodos de forma a garantir a estabilidade do sistema através da modulação adequada da válvula choke, reduzindo ou mesmo eliminando o comportamento oscilatório da produção, mantendo a válvula choke na maior abertura possível, trazendo desta forma, ganhos econômicos através do aumento da produtividade do poço. Para a análise do sistema, utilizou-se um modelo dinâmico simplificado encontrado na literatura. Este modelo foi escolhido por reproduzir adequadamente o escoamento em golfadas severas, causado pela presença de trecho horizontal descendente na linha de produção. O sistema foi também modelado no OLGA ${ }^{\circledR}$, sendo este, um software comercial com uso bastante disseminado na indústria de petróleo e com grande aplicação em simulações que exigem uma análise do comportamento transiente do problema. A avaliação da eficácia dos métodos de supressão das golfadas foi realizada através de duas abordagens distintas. Inicialmente, utilizou-se um controlador PI adaptativo com capacidade de operação em diferentes regiões de aberturas da válvula choke. Por fim, utilizou-se um Regulador Linear Quadrático associado a um observador de estados com filtro de Kalman Unscented com o objetivo de verificar como o sistema se comportaria em caso de necessidade de um observador para compensar uma possível falha do sensor submarino de pressão. Em ambos os casos, notou-se redução nas oscilações devido a golfadas do poço. As soluções foram implementadas através da integração entre o modelo do $\mathrm{OLGA}^{\circledR} \mathrm{e}$ os algoritmos desenvolvidos no MATLAB utilizando servidor OPC.

Palavras-Chave: Controle avançado de golfada severa, óleo e gás, produção offshore, válvula choke, modelos de escoamento por golfadas. 


\begin{abstract}
ASHIKAWA, F.H. Slugs mitigation in offshore systems by simplified dynamic model. São Paulo, 2017. MSc Thesis (Master in Chemical Engineering at Polytechnic School of the University of Sao Paulo), Brazil, 2017.

Nowadays, there are numerous offshore installations operating in unstable flow regimes. Such unstable flow is the result of oversized diameters of production lines or low flow wells. It should be noticed that often the intermittent system occurs at the end of the productive life of wells due to the natural decline of the reservoir, which causes a reduction in oil field production. Such instability brings difficulties in offshore production, it can cause damage to the platform equipment or production losses. In this context, it is necessary to consider means to reduce or remove this type of flow regime. The objective of this work is to develop methods to ensure system stability by adequate modulation of choke valve, reducing or even eliminating the oscillatory behavior of the production, with the biggest choke valve opening possible, bringing economic gains by increasing well production The system analysis was carried out using a simplified dynamic model found in literature. This model was chosen by appropriately reproduction of severe slug flow, caused by the presence of downward horizontal section in the production line. The problem was also modeled on OLGA ${ }^{\circledR}$, which is a commercial software with widespread use in the oil industry and large application in simulations that require a transient behavior analysis. The evaluation of the slugs suppression methods was carried out through two different approaches. First, an adaptive PI controller with capability to operate in different regions of choke valve openings was used. Finally, a Quadratic Linear Regulator associated to an Unscented Kalman Filter was used to verify how the system would behave in the event of a possible failure of subsea pressure sensor. In both cases, there was a reduction in oscillations due to slugs. The solutions were implemented through communication between the $O L G A^{\circledR}$ model and the control algorithms developed in MATLAB using OPC server.
\end{abstract}

Keywords: Riser slug advanced control, oil and gas, offshore production, choke valve, slugging flow models 


\section{GLOSSÁRIO E SIGLAS}

Gas lift

Flowline

Pipeline

Riser

Slug

Subsea

Topside

Válvula Choke

Wellhead

ABB

EKF

LQR

PIG

SP

SPKF

UKF
Injeção de gás com o objetivo de reduzir as perdas de carga no escoamento e aumentar a produtividade do poço

Trecho horizontal das linhas submarinas dos poços

Sinônimo de Flowline

Trecho vertical das linhas submarinas dos poços

Golfada

Localização abaixo do nível do mar

Localização acima do nível do mar (oposto de subsea)

Válvula de controle de vazão

Cabeça do poço

Asea Brown Boveri S.A.

Extended Kalman Filter

Linear-quadratic regulator

"Pipeline Inspection Gauge"

Sigma Point

Sigma Point Kalman Filter

Unscented Kalman Filter 


\section{SIMBOLOS}

A Área da seção transversal do tubo

\begin{tabular}{|c|c|}
\hline$A_{G}, A_{L}$ & Áreas da seção transversal ocupadas pelo gás e pelo líquido \\
\hline$A_{p}, A_{r}$ & Áreas do pipeline e do riser \\
\hline$A_{g}, A_{l}$ & Área livre para o escoamento de gás e de líquido na base do riser \\
\hline$C_{v 1}$ & Constante da válvula choke de produção \\
\hline$d_{1}, d_{2}$ & Distúrbios de outros poços na malha de drenagem \\
\hline$D_{p}, D_{r}, D_{w}$ & Diâmetros do pipeline, do riser e do poço \\
\hline$D_{i}$ & Diâmetro interno da seção transversal circular \\
\hline$e$ & Erro de saída \\
\hline$E$ & Energia interna por unidade de massa \\
\hline$f()$ & Função não linear do sistema do processo \\
\hline$F$ & Matriz Jacobiana das derivadas parciais de $f($ ) em relação a $x$ \\
\hline gor & Razão mássica gás óleo \\
\hline$G_{g}$ & Fonte de massa da fase gás \\
\hline$G_{L}$ & Fonte de massa da fase líquida \\
\hline$G_{D}$ & Fonte de massa da fase dispersa de gotículas de líquido. \\
\hline$G_{f}$ & Fonte interna \\
\hline$h$ & Elevação \\
\hline$h_{L}$ & Altura ocupada pelo líquido \\
\hline$h()$ & Função não linear do sistema de medição \\
\hline $\bar{h}$ & Nível de líquido no ponto mais baixo do pipeline \\
\hline$h_{d}$ & Abertura ao fluxo na base do riser \\
\hline$H$ & Matriz Jacobiana das derivadas parciais de $\mathrm{h}($ ) em relação a $x$ \\
\hline$H_{S}$ & Entalpia da fonte de massa \\
\hline$J$ & Função custo do controlador LQR \\
\hline
\end{tabular}




\begin{tabular}{|c|c|}
\hline$k$ & Fator de escala \\
\hline$K$ & Ganho de adaptação \\
\hline$K_{g}$ & Coeficiente para a vazão de gás na base do riser \\
\hline$K_{l}$ & Coeficiente para a vazão de líquido na base do riser \\
\hline$K_{h}$ & Fator de correção do nível de líquido no pipeline \\
\hline$K_{e}$ & Matriz de ganho do observador \\
\hline$K_{l q r}$ & Ganho do controlador LQR \\
\hline$L_{p}, L_{r}, L_{w}$ & Comprimentos do pipeline, do riser e do poço \\
\hline$m_{g, r}$ & Massa de gás no riser \\
\hline$m_{g, p}, m_{l, p}$ & Massas de gás no pipeline e de líquido no pipeline \\
\hline$m_{g, r}, m_{l, p}$ & Massa de gás no riser e de líquido no riser \\
\hline$n$ & Vetor de variáveis aleatórias \\
\hline$p$ & Pressão \\
\hline $\mathrm{R}$ & Solução definida simétrica positiva da equação de Riccatti. \\
\hline$P$ & Matriz de covariância dos estados \\
\hline$P^{x y}$ & Matriz de correção cruzada entre $x$ e $y$ \\
\hline$P_{S}$ & Pressão de separação \\
\hline$P_{r t}$ & Pressão no topo do riser \\
\hline$P_{\text {res }}$ & Pressão do reservatório \\
\hline$P_{b h}, P_{w h}$ & Pressão no fundo e na cabeça do poço \\
\hline$P_{\text {in,nom }}$ & Pressão de entrada nominal em regime permanente do pipeline \\
\hline$P I$ & Índice de produtividade \\
\hline Q & Matriz de peso do controlador LQR para os estados $x$ \\
\hline$Q$ & Matriz de covariância do ruído de processo \\
\hline $\mathrm{R}$ & Matriz de peso do controlador LQR para a entrada $u$ \\
\hline$R$ & Matriz de covariância do ruído de medição \\
\hline$R_{S}$ & Fração mássica de gás em condições de equilíbrio \\
\hline
\end{tabular}




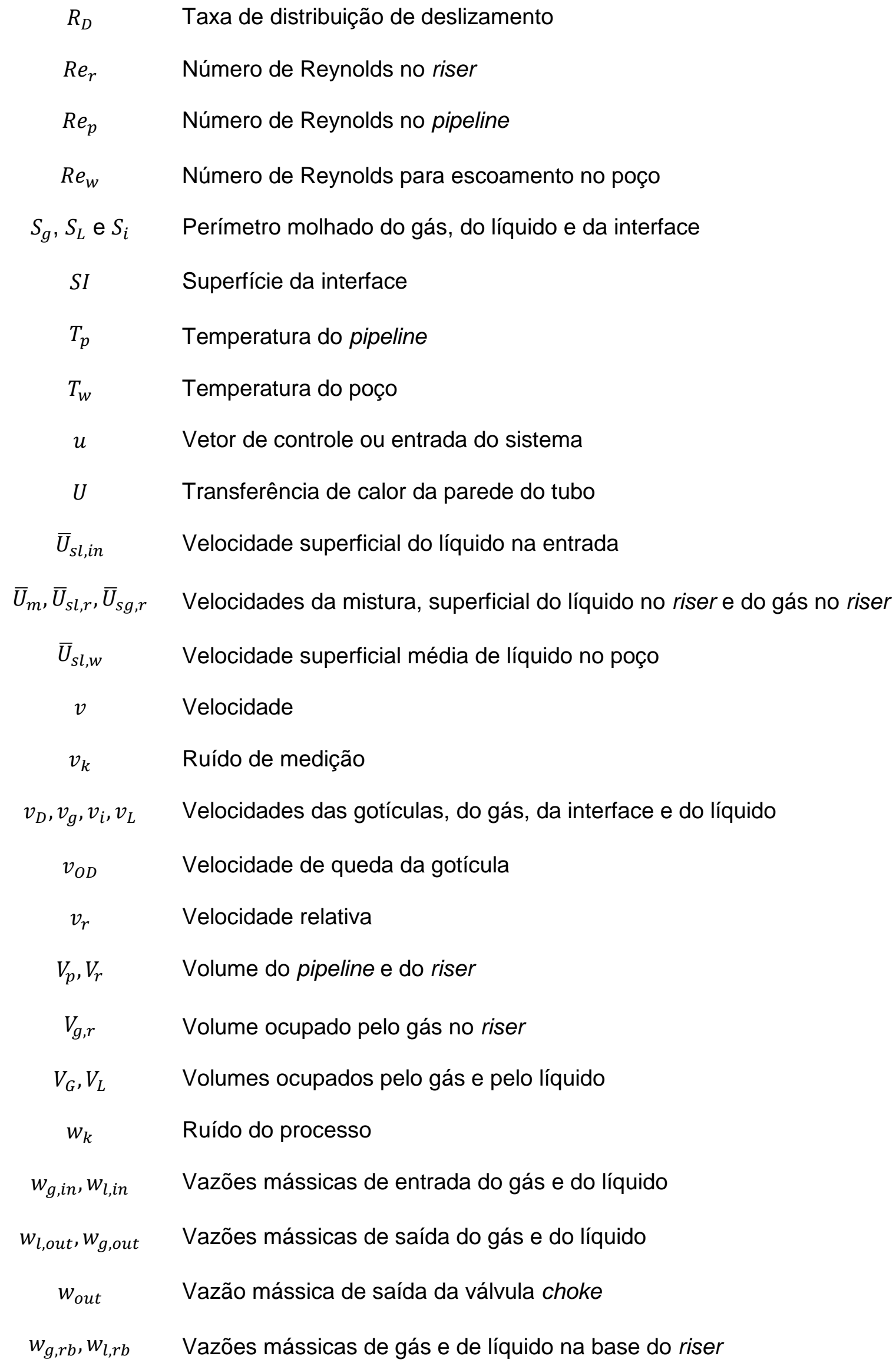




\begin{tabular}{|c|c|}
\hline$w_{r}$ & Vazão mássica do reservatório \\
\hline$w_{\text {nom }}$ & Vazão mássica nominal do reservatório \\
\hline$w_{g, w h}, w_{l, w h}$ & Vazão mássica de gás e líquido na cabeça do poço \\
\hline$W$ & Matriz Jacobiana das derivadas parciais de $\mathrm{f}(\mathrm{)})$ em relação a $w$ \\
\hline$x$ & Vetor de estados do sistema \\
\hline$\tilde{x}$ & Estado estimado \\
\hline$y$ & Vetor de resposta ou saída do sistema \\
\hline$z$ & Abertura da válvula choke \\
\hline$z^{*}$ & Abertura crítica da válvula choke \\
\hline$z 1$ & Abertura normalizada da válvula choke \\
\hline$\alpha_{l, w t}$ & Fração volumétrica no topo do poço \\
\hline$\alpha_{g, w t}^{m}$ & Fração mássica do gás no topo do poço \\
\hline$\alpha_{g}, \alpha_{L}, \alpha_{D}$ & Frações volumétrica do gás, do líquido e das gotículas de líquido \\
\hline$\alpha_{l, r t}$ & Fração volumétrica de líquido no topo do riser \\
\hline$\alpha_{l, r b}$ & Fração volumétrica de líquido na base do riser \\
\hline$\alpha_{l, r t}^{m}$ & Fração mássica de líquido no topo do riser \\
\hline$\alpha_{l}$ & Fração volumétrica de líquido \\
\hline$\alpha_{l}^{m}$ & Fração mássica de líquido na seção do pipeline \\
\hline $\bar{\alpha}_{l, p}^{m}$ & Fração mássica média de líquido na seção do pipeline \\
\hline $\bar{\alpha}_{l, p}$ & Fração volumétrica média de líquido na seção do pipeline \\
\hline $\bar{\alpha}_{l, w}$ & Fração volumétrica média de líquido no poço \\
\hline $\bar{\alpha}_{l, r}$ & Fração volumétrica média de líquido no riser \\
\hline$\beta$ & Inclinação do tubo em relação a um plano horizontal \\
\hline$\Delta P_{f p}$ & Perdas por fricção no pipeline \\
\hline$\Delta P_{f r}$ & Perdas por fricção no riser \\
\hline$\Delta P_{f w}$ & Perda de carga no poço devido ao atrito \\
\hline
\end{tabular}




\begin{tabular}{|c|c|}
\hline$\theta$ & Parâmetros da planta \\
\hline$\theta$ & Inclinação do pipeline \\
\hline$\lambda_{p}$ & Fator de fricção no pipeline \\
\hline$\lambda_{r}$ & Fator de fricção no riser \\
\hline$\lambda_{w}$ & Fator de atrito no poço \\
\hline$\mu$ & Viscosidade do líquido \\
\hline$\rho$ & Massa específica \\
\hline$\rho_{r t}$ & Densidade da mistura no topo do riser \\
\hline$\rho_{l}$ & Densidade do líquido \\
\hline$\rho_{g, \mathrm{r}}$ & Densidade do gás no riser \\
\hline$\rho_{g}$ & Densidades do gás \\
\hline$\rho_{g, p}$ & Densidade do gás no pipeline \\
\hline $\bar{\rho}_{g, p}$ & Densidade média do gás no pipeline \\
\hline$\rho_{g, r}$ & Densidade do gás no riser \\
\hline $\bar{\rho}_{m, r}$ & Densidade média da mistura no riser \\
\hline $\bar{\rho}_{m, w}$ & Densidade média da mistura bifásica \\
\hline$\rho_{g, w}$ & Densidade da fase gás no poço \\
\hline$\rho_{w t}$ & Densidade da mistura no poço \\
\hline$\sigma$ & Conjunto de pontos sigma \\
\hline$\tau_{G}, \tau_{L}, \tau_{I}$ & Atrito do gás na parede, do líquido na parede e na interface \\
\hline$\Psi$ & Vetor de parâmetros do controlador adaptativo \\
\hline$\psi_{g}$ & Taxa de transferência de massa entre as fases \\
\hline$\psi_{e}$ & Taxa de arrastamento \\
\hline$\psi_{d}$ & Taxa de deposição \\
\hline
\end{tabular}




\section{LISTA DE FIGURAS}

Figura 2-1 - Esquemático de um poço de petróleo offshore. 22

Figura 2-2 - Padrões de fluxo multifásico em um duto horizontal para diferentes velocidades de escoamento.

Figura 2-3 - Padrões de fluxo multifásico em um duto vertical para diferentes velocidades de escoamento. 24

Figura 2-4 - Esquemático de um sistema sujeito ao fenômeno de golfada severa. 26

Figura 2-5 - Ciclo de formação de golfadas severas. 27

Figura 2-6 - Diagrama de bifurcação. 28

Figura 3-1 - Diagrama de blocos genérico de um controle adaptativo. 30

Figura 3-2 - Sistema de controle adaptativo por escalonamento de ganhos . 31

Figura 3-3 - Controle adaptativo por modelo de referência .... 32

Figura 3-4 - Controle adaptativo auto-sintonizado. 34

Figura 3-5 - Observador de estado de ordem plena..... 38

Figura 3-6 - Sistema de controle realimentado por estado observado. 40

Figura 3-7 - Diagrama de blocos do sistema com controlador-observador. 42

Figura 3-8 - Sistema de controle realimentado por estado observado de ordem mínima .... 42

Figura 3-9 - Estrutura geral do filtro de Kalman.... 47

Figura 3-10 - Princípio do filtro de Kalman Unscented 50

Figura 3-11 - Seção transversal do escoamento. 55

Figura 3-12 - Perfil lateral de escoamento estratificado. 55

Figura 3-13 - Representação simplificada do escoamento (Sem bloqueio de líquido)..... 63

Figura 3-14 - Representação simplificada do bloqueio de líquido na base do riser, levando a golfada severa. 63

Figura 3-15 - Esquemático de um sistema poço-pipeline-riser. 71

Figura 3-16 - Modelo do OLGA® para o sistema pipeline-riser 75

Figura 3-17 - Curva de fração mássica de gás $x$ temperatura do fluido. 76

Figura 3-18 - Modelo do OLGA® para o sistema poço-pipeline-riser .... 77

Figura 4-1 - Comportamento das pressões na entrada do pipeline (PIPE-1) e no topo do riser (PIPE-5) para uma abertura crescente da válvula choke 78

Figura 4-2 - Oscilação das pressões de entrada do pipeline e de topo do riser para diferentes aberturas da válvula choke

Figura 4-3 - Diagrama de bifurcação - pressão na entrada do pipeline x abertura da válvula choke. 
Figura 4-4 - Diagrama de bifurcação - pressão no topo do riser $\mathrm{x}$ abertura da válvula choke

Figura 4-5 - Oscilação das pressões do fundo do poço e no topo do riser para diferentes aberturas da válvula choke

Figura 4-6 - Diagrama de bifurcação - pressão no fundo do poço x abertura da válvula choke

Figura 4-7 - Diagrama de bifurcação - pressão no topo do riser $x$ abertura da válvula choke

Figura 4-8 - Pressões P1 e P2, vazão volumétrica Q e vazão mássica W para a válvula choke com abertura de $5 \%$

Figura 4-9 - Pressões P1 e P2, vazão volumétrica $Q$ e vazão mássica W para a válvula choke com abertura de $10 \%$.

Figura 4-10 - Pressões P1 e P2, vazão volumétrica $Q$ e vazão mássica $W$ para a válvula choke com abertura de $20 \%$

Figura 4-11 - Resposta ao degrau e diagrama de Bode para a função de transferência de z para $\mathrm{P} 1$. 90

Figura 4-12 - Resposta ao degrau de P1 para aberturas da válvula choke variando de 10\% a $50 \%$ utilizando um controlador PI de ganho estático

Figura 4-13 - Resposta ao degrau de P1 para aberturas da válvula choke variando de $10 \%$ a $50 \%$ utilizando um controlador PI adaptativo 92

Figura 4-14 - Variação do ganho proporcional e do tempo integral do controlador PI adaptativo para diferentes aberturas da válvula choke

Figura 4-15 - Comportamento da pressão de entrada do pipeline P1 e abertura da válvula choke com a utilização do controlador adaptativo PI para diferentes setpoints de pressão. .94

Figura 4-16 - Pressões P1 e P2 com controlador LQR + Filtro de Kalman Unscented para diferentes aberturas da válvula choke. 96

Figura 4-17 - Simulação do OLGA® para as pressões P1 e P2 com o controlador LQR + Filtro de Kalman Unscented 97

Figura A-1 - Tela de configurações básicas do servidor OPC no OLGA ${ }^{\circledR}$......................... 106

Figura A-2 - Tela de configuração das variáveis a serem expostas no servidor OPC........ 107

Figura A-3 - Barra de tarefas para início da simulação em "batch" .................................. 107

Figura A-4 - Tela de status da simulação ................................................................... 108

Figura A-5 - Tela de configuração do bloco do Simulink "OPC Configuration”................... 108

Figura A-6 - Blocos do Simulink para leitura e escrita de variáveis do servidor OPC ........ 109 
Figura B-1 - Diagrama Simulink do controlador PI adaptativo utilizando o modelo linearizado do MATLAB 110

Figura B-2 - Diagrama Simulink do controlador PI adaptativo utilizando o modelo do OLGA® 111

Figura C-1 - Diagrama Simulink do regulador linear quadrático (LQR) com Filtro de Kalman Unscented (UKF) utilizando o modelo linearizado do MATLAB 112

Figura C-2 - Diagrama Simulink do regulador linear quadrático (LQR) com Filtro de Kalman Unscented (UKF) utilizando o modelo do OLGA® 113 


\section{LISTA DE TABELAS}

Tabela 1 - Substituições para a equação do observador de ordem reduzida ..................... 44

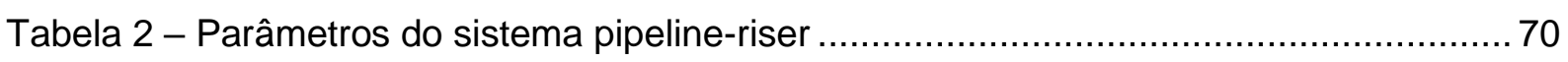

Tabela 3 - Parâmetros adicionais para o sistema poço-pipeline-riser................................ 74

Tabela 4 - Dados da geometria do sistema pipeline-riser modelado no OLGA ${ }^{\circledR}$.................. 75

Tabela 5 - Dados da geometria do sistema poço-pipeline-riser modelado no OLGA ${ }^{\circledR} \ldots . . . . .77$ 


\section{SUMÁRIO}

1. INTRODUÇÃO

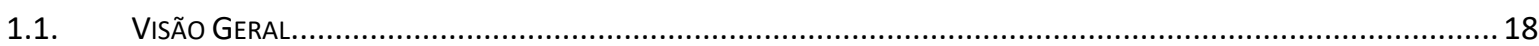

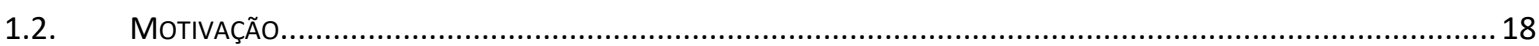

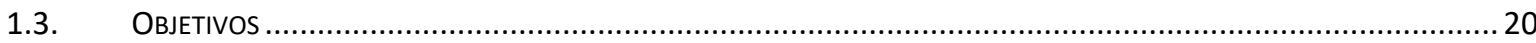

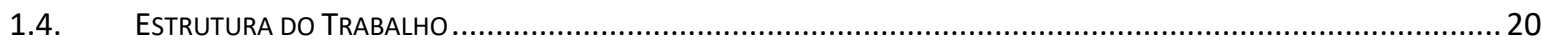

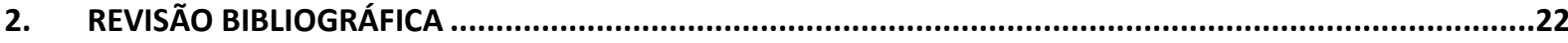

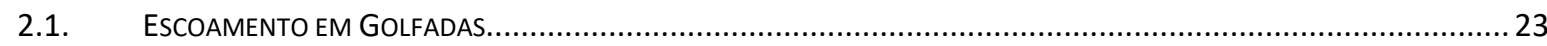

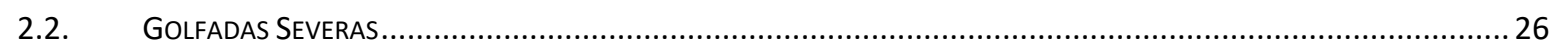

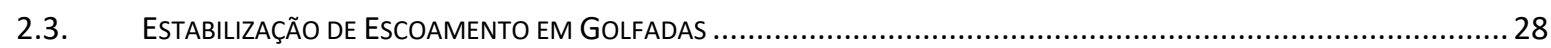

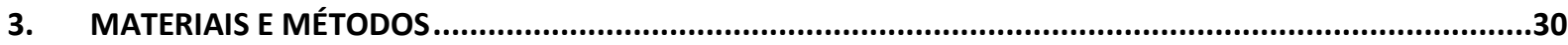

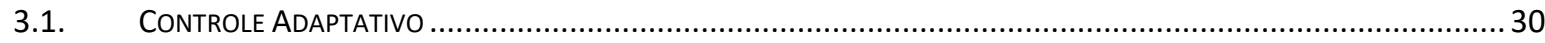

3.1.1. Controle Adaptativo com Escalonamento de Ganho ................................................................ 31

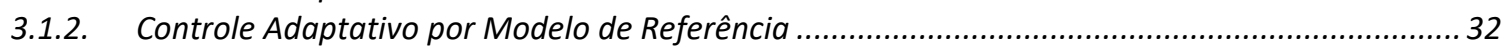

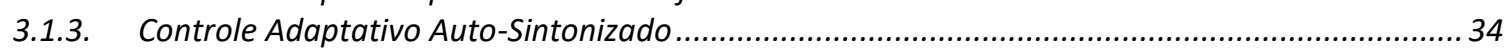

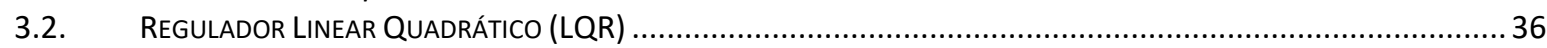

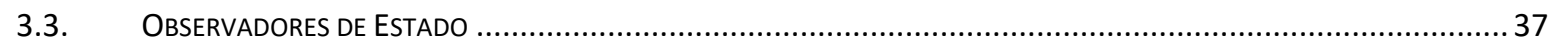

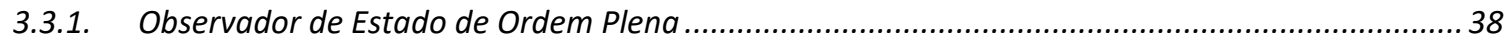

3.3.2. Observador de Ordem Reduzida …….......................................................................... 42

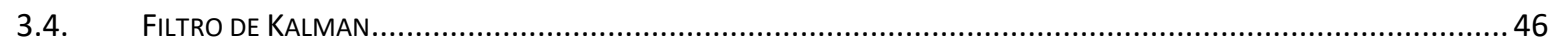

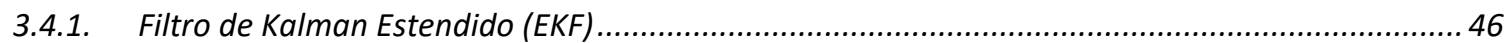

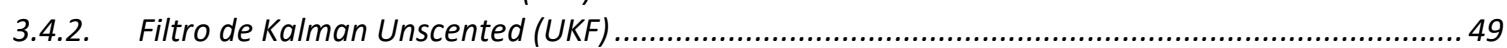

3.5. SIMULADOR OLGA

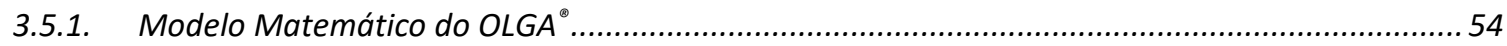

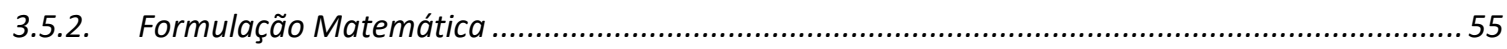

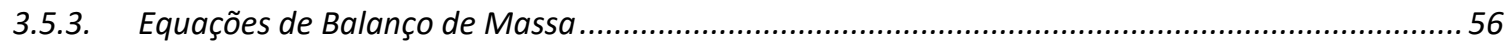

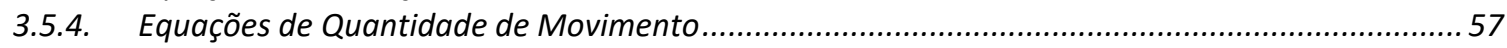

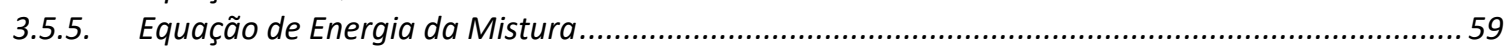

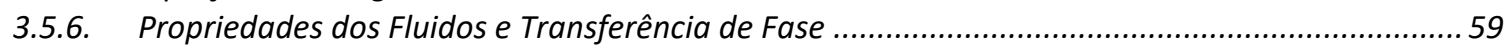

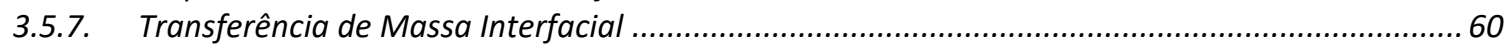

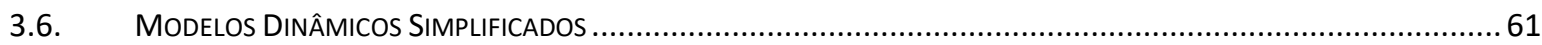

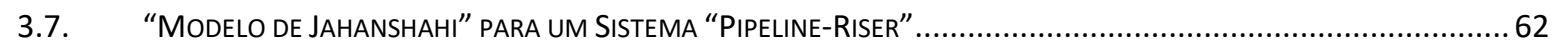

3.7.1. Equações de Balanço de Massa para um Sistema "Pipeline-Riser"..............................................62

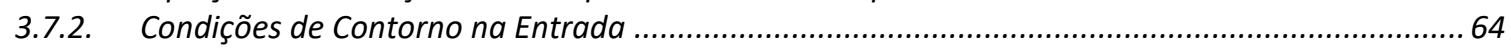

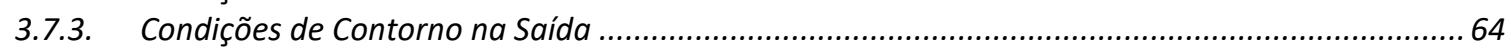

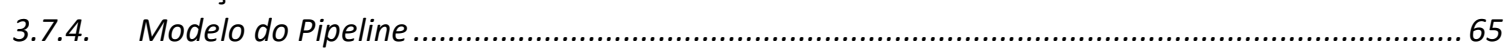

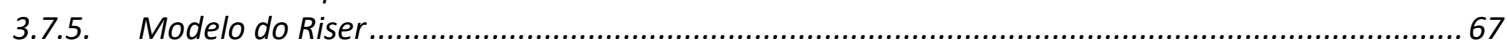

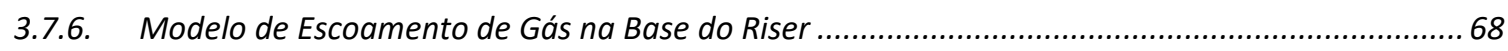

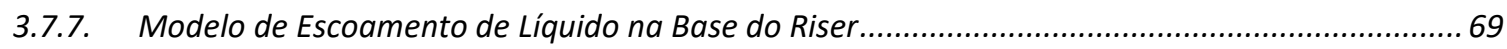

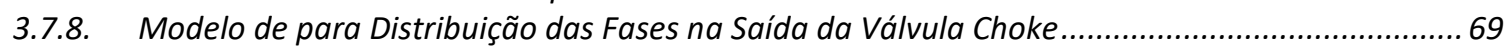

3.8. "MODELO DE JAHANSHAHI" PARA UM SISTEMA “POÇO-PIPELINE-RISER" .................................................... 71

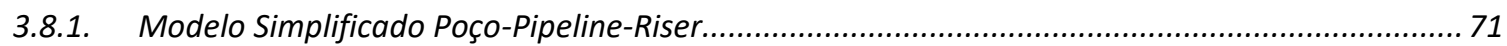

3.9. MODELAGEM DO SISTEMA NO SIMULADOR OLGA

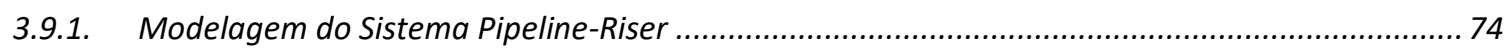

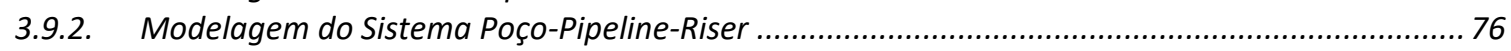

4. RESULTADOS E DISCUSSÃO ..................................................................................................................78

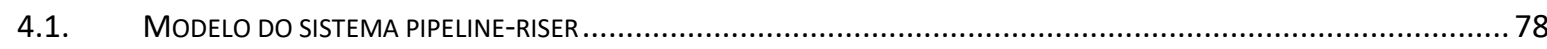

4.1.1. Pressões de Entrada e Saída e Diagrama de Bifurcação do Pipeline-Riser no OLGA ${ }^{\circ}$.......................... 78

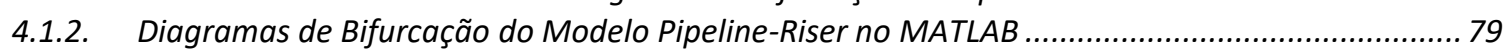

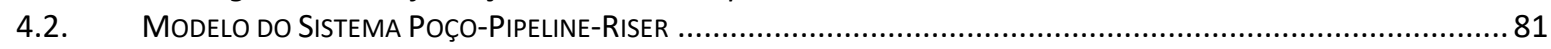


4.2.1. Pressões de Entrada e Saída e Diagrama de Bifurcação do Poço-Pipeline-Riser no OLGA ...........81

4.2.2. Diagramas de Bifurcação pelo Modelo Poço-Pipeline-Riser no MATLAB ....................................82

4.3. Algoritmos de Controle Para MitigaÇÃo de Golfadas .................................................................. 84

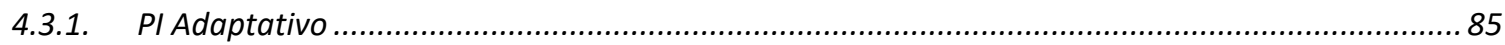

4.3.2. Regulador Linear Quadrático com Filtro de Kalman Unscented ..............................................94

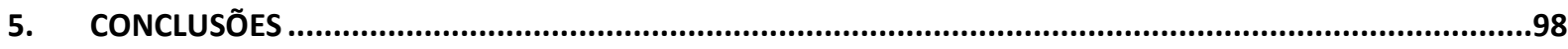

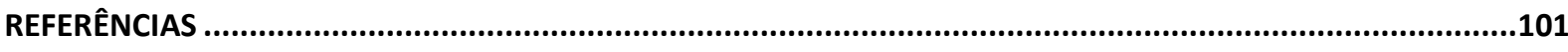

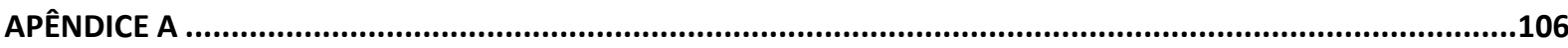

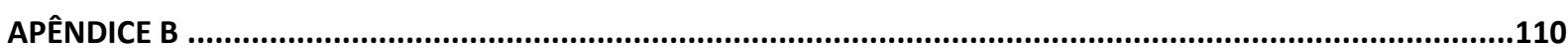

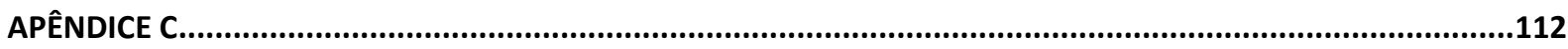

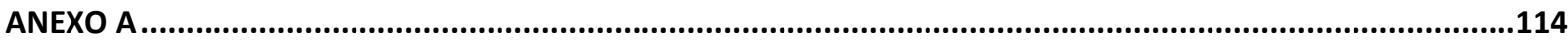

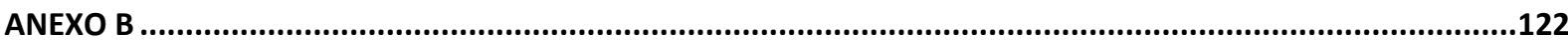




\section{INTRODUÇÃO}

\subsection{Visão Geral}

A exploração do petróleo se iniciou em jazidas de pequena profundidade que demandavam menores esforços e pouca tecnologia. Entretanto, à medida que estes campos superficiais se esgotaram, foi necessário um maior desenvolvimento tecnológico de forma a possibilitar a exploração de reservas mais profundas. No Brasil há um desafio adicional, pois, as maiores reservas de petróleo se encontram em alto mar (offshore). Com o avanço tecnológico, a fronteira exploratória se deslocou para lâminas d'água cada vez mais profundas. Nestas condições, a tendência é que o escoamento de petróleo esteja sujeito a temperaturas menores, o que possibilita a formação de bloqueios nas linhas pela formação de hidratos e/ou parafinas, além disso, há uma maior probabilidade de o escoamento ser intermitente (JAHANSHAHI, 2013).

Quando o regime de escoamento é em golfadas, ou seja, é composto por severas variações na produção de óleo e gás, tem-se diversos problemas na plataforma, como: redução de eficiência na separação dos fluidos, possíveis perdas de produção ocasionadas por paradas não programadas (shutdown) ou restrição de produção por capacidade limitada da planta de processo, causada pelos picos de vazão dos poços.

Devido a estes desafios, o estudo do regime de escoamentos multifásicos na produção offshore e a mitigação de instabilidades é cada vez mais importante para se evitar perdas de produção e aumentar a produtividade dos campos de petróleo.

\subsection{Motivação}

Conforme descrito anteriormente, as golfadas representam um problema que não pode ser ignorado, pois produz consequências bastante prejudiciais à produção de petróleo. As formas mais comuns de contornar este problema estão descritas a seguir: 
- Aumentar o volume do separador de produção, ainda na etapa de projeto, de modo que o mesmo consiga absorver as variações decorrentes da produção em golfadas. Esta solução possui como desvantagem uma maior necessidade de investimentos. Há ainda um outro fator limitante: uma plataforma de produção offshore possui, muitas vezes, espaço bastante limitado, portanto, a opção por um equipamento de maior dimensão pode ser tecnicamente inviável (BENDIA, 2013).

- Utilizar a injeção de gas lift (Figura 2-1), pois com uma vazão adequada de gás injetado, pode-se alterar o regime de escoamento, através da mudança na relação de velocidades das fases de gás e líquido escoado. Este método tem algumas desvantagens, como o aumento do custo de compressão para suprir a demanda adicional de gás (SCHMIDT; BRILL; BEGGS, 1980). Outra desvantagem é que nem sempre há capacidade suficiente de compressão de gás para suprir a vazão ótima de injeção em todos os poços (Problema de otimização) e a partir de um certo patamar de vazão, o aumento de perda de carga por fricção é predominante e causa perdas de produção.

- Restringir manualmente a válvula choke (Figura 2-1) do poço que apresentar comportamento oscilatório. Esta solução foi uma das primeiras a serem propostas para evitar o escoamento em golfadas (YOCUM, 1973). A restrição desta válvula pode reduzir ou, até mesmo, eliminar o comportamento oscilatório do poço. Porém, ao adotar esta solução, aumenta-se a contrapressão sobre o poço e, consequentemente, há uma perda de produção decorrente desta alternativa.

- Uma opção à restrição manual da válvula choke é a modulação automática da mesma. Há diversos estudos que comprovam que a modulação adequada da válvula choke pode eliminar as oscilações sem causar perdas de produção. A manipulação ativa desta válvula pode inclusive aumentar a produtividade dos poços, reduzindo a pressão média de escoamento (GODHAVN; FARD; FUCHS, 2004). Estes controladores normalmente utilizam como variável manipulada a abertura do choke topside e, como variável controlada, a pressão na base do riser. 
Na prática, os sistemas de controle anti-golfadas que modulam a válvula choke sofrem com limitações de robustez, ou seja, o sistema se torna instável após algum tempo devido a, por exemplo, mudanças na vazão dos poços e/ou na planta de processo. Com a perda de eficiência do controlador, os operadores tendem a desligar o mesmo e utilizar o fechamento manual da válvula choke. Portanto, é importante que sejam obtidas soluções robustas para este problema.

Um agravante do sistema sujeito a golfadas é que o mesmo se caracteriza por ser altamente não linear, sendo que os ganhos do controlador mudam drasticamente para diferentes condições de operação. Deve-se também lidar com o atraso de controle (time delay) decorrente de linhas de produção com grande comprimento, o que dificulta ainda mais a estabilização do sistema.

\subsection{Objetivos}

O objetivo deste trabalho é desenvolver métodos de forma a garantir a estabilidade do sistema através da modulação adequada da válvula choke, reduzindo ou mesmo eliminando o comportamento oscilatório da produção, mantendo a válvula choke na maior abertura possível, trazendo desta forma, ganhos econômicos através do aumento da produtividade do poço.

\subsection{Estrutura do Trabalho}

No capítulo 1, são introduzidos conceitos sobre o escoamento intermitente, em especial o composto pelo regime de golfadas. Foi descrita também a motivação para realização deste trabalho, através dos ganhos potenciais da modulação adequada da válvula choke na supressão das instabilidades.

No capítulo 2, são apresentados os objetivos do trabalho.

O capítulo 3 apresenta a revisão bibliográfica do tema de forma a identificar os estudos mais relevantes da área e o estado da arte no que diz respeito aos métodos de mitigação de golfadas em plataformas de petróleo. Aqui consta também uma breve 
revisão bibliográfica englobando os conceitos de Controle Adaptativo, de Observadores de Estado e Filtros de Kalman formando a base teórica que será utilizada na implementação dos controladores.

No capítulo 4, é apresentado um modelo dinâmico simplificado encontrado na literatura que representa adequadamente o problema de golfadas severas através de sistemas poço-pipeline e poço-pipeline-riser.

No capítulo 5, é exposto o desenvolvimento da metodologia de supressão de golfadas utilizando algoritmos de controle, tomando como base o modelo dinâmico simplificado apresentado no capítulo 4.

No capítulo 6, são apresentadas as conclusões obtidas neste trabalho. Engenharia Química, 2016, Fortaleza, CE. XXI CIBEQ. São Paulo: ABEQ, 2016. 


\section{REVISÃO BIBLIOGRÁFICA}

Os regimes de escoamento com golfadas severas ocorrem em sistemas formados por flowlines/risers que transportam uma mistura multifásica (óleo e gás) do leito marinho até a superfície (TAITEL, 1986).

Este tipo de regime de escoamento é caracterizado por apresentar altas oscilações nas vazões e pressões. Este fluxo intermitente causa diversos problemas para os equipamentos da plataforma. Portanto, são necessários métodos efetivos para lidar com as mesmas e diversos estudos foram desenvolvidos para tentar prevenir estas ocorrências (HAVRE; DALSMO, 2002).

A solução mais simples é a restrição manual da válvula choke de topside, porém, esta manobra reduz a vazão de produção, especialmente em campos em que a pressão de reservatório é relativamente baixa. Portanto, é desejável uma solução que estabilize o fluxo e garanta a maior produção possível.

Na Figura 2-1, apresenta-se um esquemático genérico de um sistema de produção de petróleo offshore, com o intuito de facilitar o entendimento dos termos técnicos e equipamentos citados nos próximos capítulos:

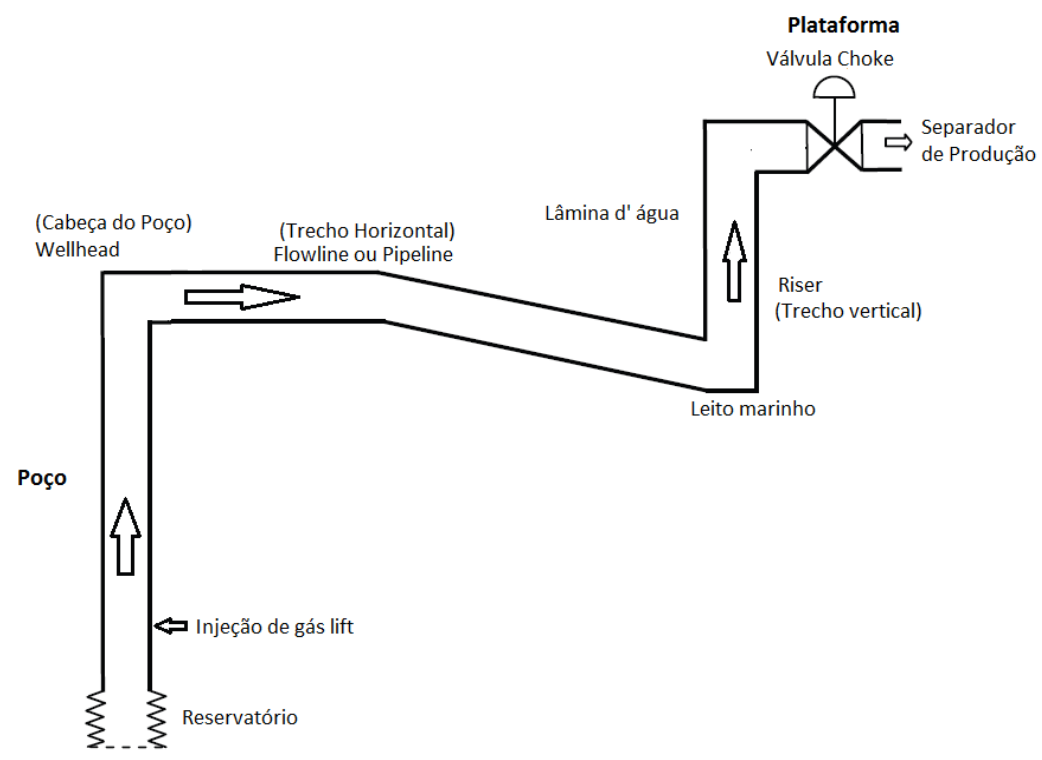

Figura 2-1 - Esquemático de um poço de petróleo offshore. 


\subsection{Escoamento em Golfadas}

O comportamento de um fluxo bifásico é muito mais complexo que um fluxo monofásico. O fluxo bifásico é constituído de um processo que envolve interações de diversas variáveis. As fases gás e líquido normalmente não fluem com a mesma velocidade no duto, devido as diferenças de densidade e viscosidade. Em um fluxo ascendente, a fase gasosa, que é menos densa e menos viscosa, tende a fluir em uma maior velocidade que a fase líquida. Por outro lado, em fluxos sujeitos a inclinações descendentes, o líquido tende a fluir em uma maior velocidade que o gás (JAHANSHAHI, 2013).

Embora existam soluções analíticas para fluxos monofásicos aplicados na indústria com resultados razoáveis, as predições para um fluxo multifásico, mesmo quando restritos a uma geometria simples são, em geral, de maior complexidade.

A empresa Total foi uma das primeiras a utilizar um sistema de controle anti-golfadas. Esta aplicação ocorreu em um duto de 16 polegadas de diâmetro localizado no Mar do Norte e sujeito a um escoamento multifásico (COURBOT, 1996). O objetivo era controlar a pressão na base do riser, manipulando a abertura da válvula choke. Em 2000, a ABB utilizou um controle similar para prevenir as golfadas entre as plataformas Hod e Valhall, também no Mar do Norte (HAVRE; STORNES; STRAY, 2000).

A Figura 2-2 mostra alguns dos padrões de fluxo multifásico em dutos horizontais para diferentes velocidades de escoamento e a Figura 2-3 mostra os padrões de escoamento para dutos verticais (BILTOFT et al., 2013), (SIVERTSEN; E. STORKAAS; SKOGESTAD, 2010). 


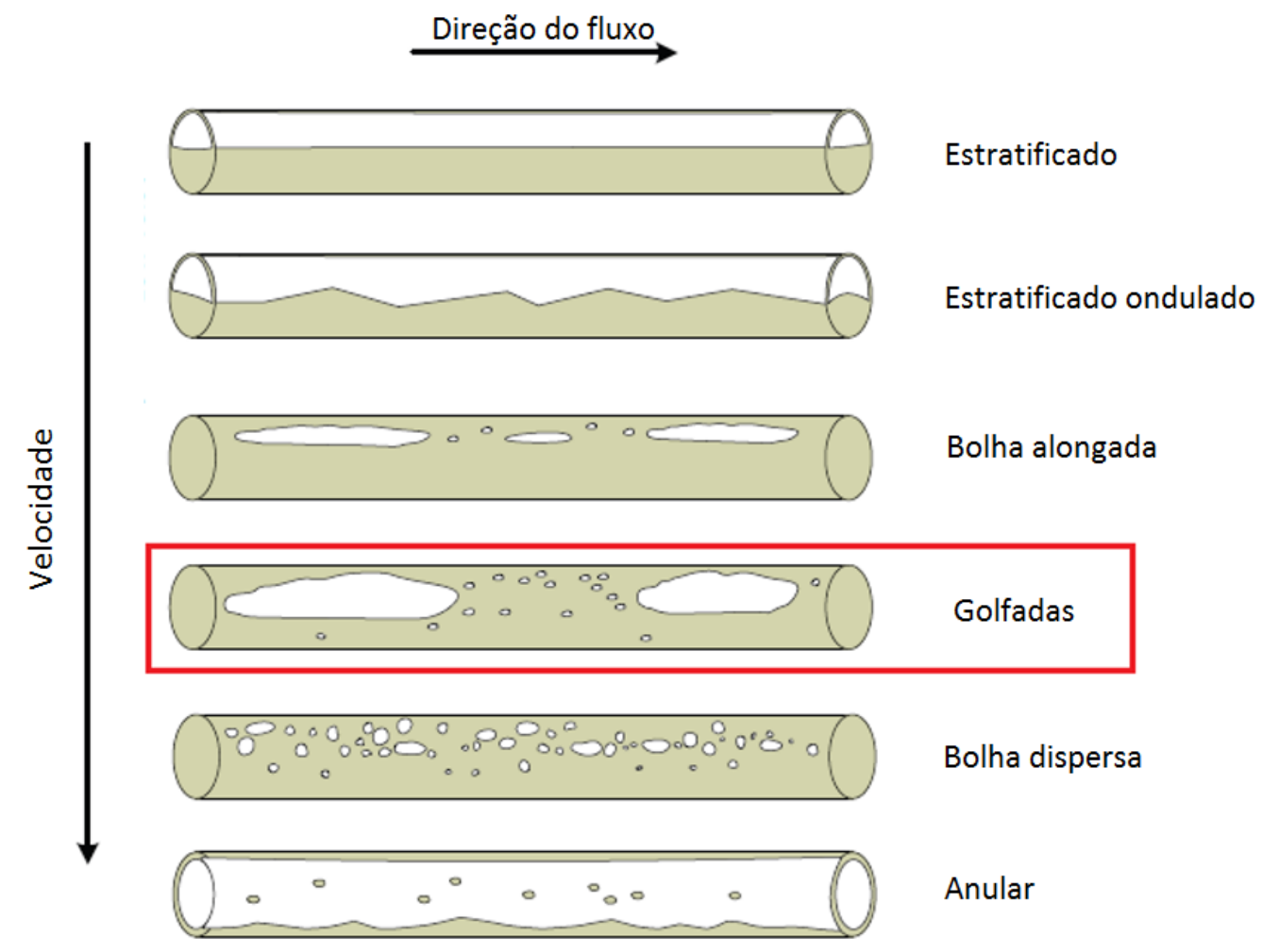

Fonte: (JAHANSHAHI, 2013)

Figura 2-2 - Padrões de fluxo multifásico em um duto horizontal para diferentes velocidades de escoamento.

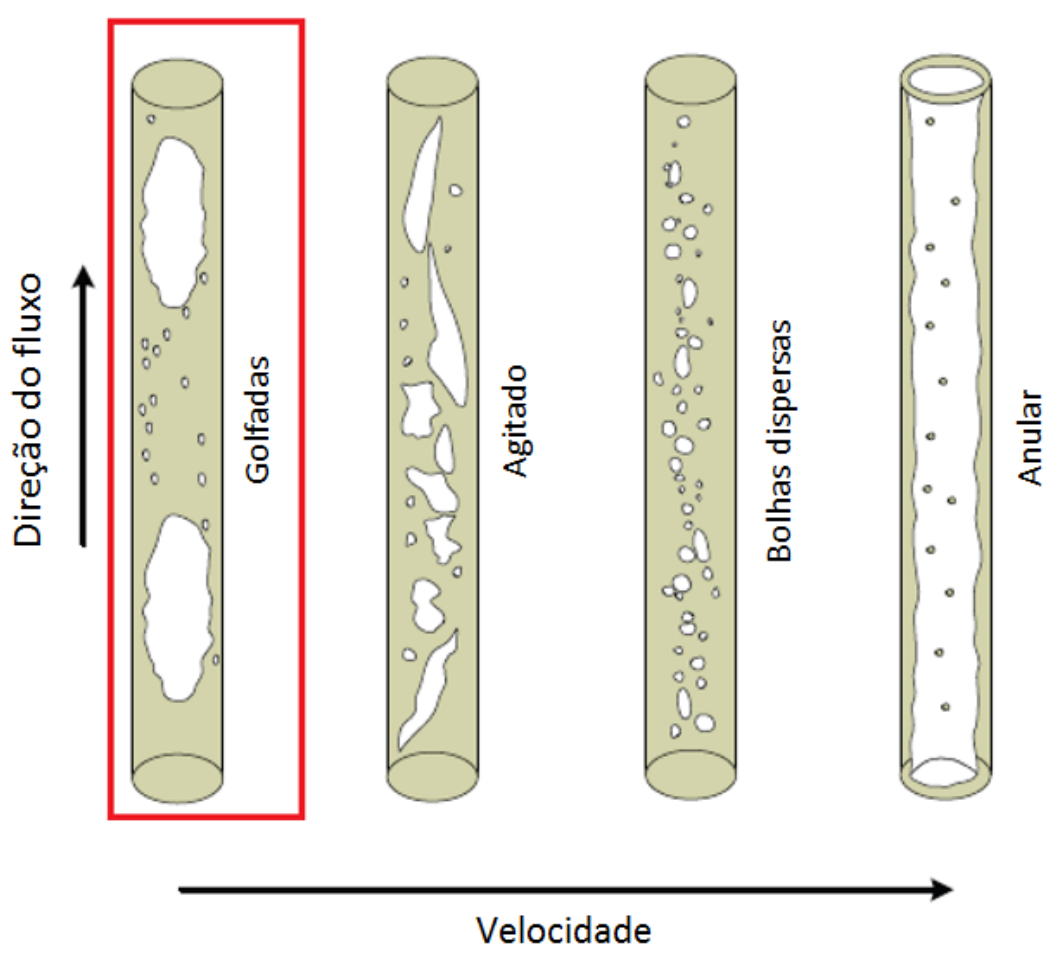

Fonte: (JAHANSHAHI, 2013)

Figura 2-3 - Padrões de fluxo multifásico em um duto vertical para diferentes velocidades de escoamento. 
Na Figura 2-2 e na Figura 2-3, o escoamento em golfadas (slug flow) foi destacado. O mesmo é caracterizado por uma sequência de bolsões de líquido (slugs) seguidos de bolsões de gás.

Algumas das causas para o escoamento em golfadas estão descritas abaixo:

1. Golfadas hidrodinâmicas (Hydrodynamic slugs) - este tipo de golfada ocorre em dutos horizontais devido à diferença de velocidades das fases líquida e gasosa. Estas golfadas são caracterizadas por apresentarem alta frequência e menor amplitude na variação das pressões e vazões envolvidas.

2. Golfada de riser (Riser slugging) ou golfada severa - este tipo de golfada é induzido pela presença de um riser vertical, geralmente associado a um trecho horizontal descendente a montante do riser associado também a uma condição de baixa vazão de escoamento. O líquido bloqueia o trecho inferior do riser, impedindo a entrada de gás no mesmo. Este bloqueio persiste até que a pressão a montante exceda a coluna hidrostática de líquido presente no riser. Este tipo de golfadas se caracteriza por ser constituído de grande volume de líquidos, causando também grandes variações de pressão na plataforma. (JAHANSHAHI, 2013).

3. Golfadas induzidas pelo terreno (Terrain-induced slugging) - induzidas pela superfície irregular do leito marinho, fazendo com que o líquido tenha tendência de acumular nas posições do duto de menor elevação, causando bloqueio do fluxo de gás no duto de forma semelhante a golfada de riser, porém com menor intensidade.

4. Golfadas causadas por manobras operacionais (Operational-based slugging) Algumas manobras e operações no poço podem causar instabilidades, um exemplo é a golfada causada pela passagem de PIG (equipamento utilizado para limpeza ou inspeção interna de dutos), esta operação gera um grande distúrbio nas vazões do poço. 
5. Golfadas causadas pelo efeito de casing heading em poços de gas lift (Casing heading slugs) - este tipo de golfada é similar a golfada de riser, porém a pressurização do gás ocorre no anular do poço e não na base do riser.

\subsection{Golfadas Severas}

A instabilidade causada pela golfada severa é um dos principais desafios de garantia de escoamento encontrados na produção offshore. A seguir é descrito em detalhes, o mecanismo de formação da golfada severa (riser slugging).

A golfada severa se forma em sistemas com fluxo multifásico (gás e líquido) com vazão relativamente baixa de produção e/ou diâmetro de duto superdimensionado. (JANSEN; SHOHAM; TAITEL, 1996). Está geralmente associada a uma geometria de linha desfavorável a montante do riser (trecho horizontal descendente), ocasionando um bloqueio de líquido na base do riser, impedindo assim, a passagem do gás. A Figura 2-4 mostra um esquemático de um sistema de produção sujeito ao bloqueio de líquido, e consequentemente ao fenômeno de golfada severa.

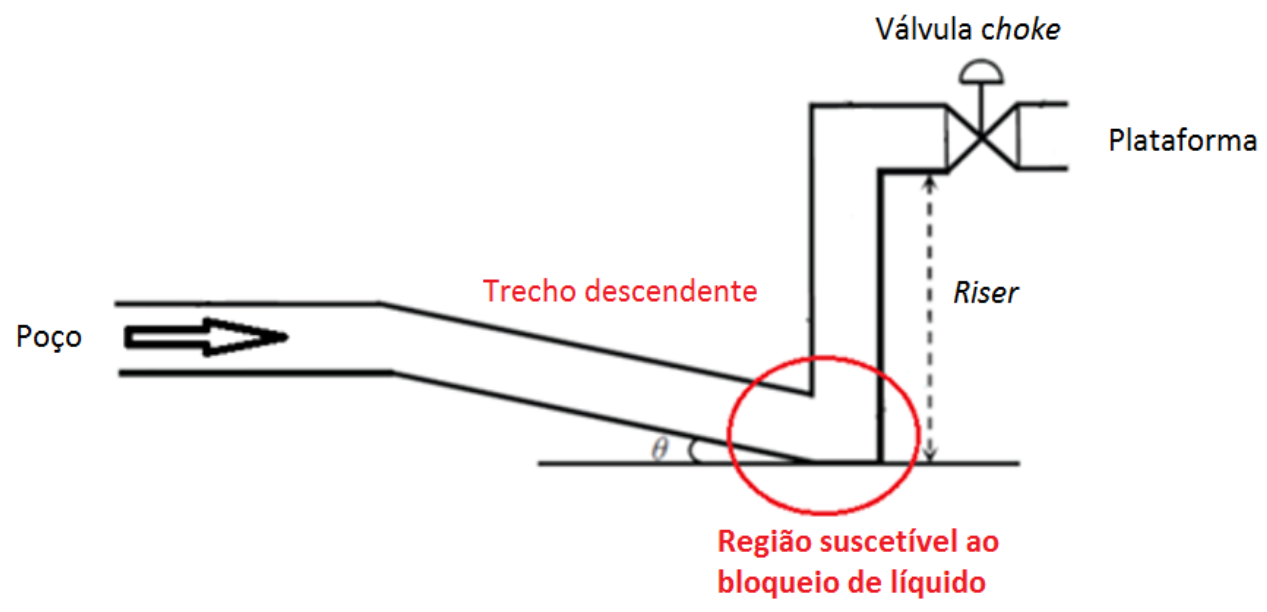

Figura 2-4 - Esquemático de um sistema sujeito ao fenômeno de golfada severa.

Este bloqueio causa um contínuo aumento da pressão a montante, até que a mesma seja suficiente para compensar a coluna hidrostática de líquido (JANSEN; SHOHAM; TAITEL, 1996).

O mecanismo de formação deste tipo de golfada pode ser descrito resumidamente em quatro passos, que estão ilustrados na Figura 2-5. 
Passo 1: O líquido acumula no ponto mais baixo do riser. Este fato ocorre apenas em escoamentos em que as velocidades de líquido e gás são baixas o suficiente para permitir este acúmulo.

Passo 2: Após a ocorrência do bloqueio, enquanto a coluna hidrostática de líquido for maior que a pressão do gás, a montante do bloqueio, ocorrerá um aumento contínuo desta pressão, causada pela compressão do gás produzido.

Passo 3: Quando o gás consegue vencer a coluna hidrostática e atinge a base do riser, ocorre um deslocamento da golfada de forma violenta. Este evento é causado pela rápida expansão do gás e pela redução da coluna hidrostática a medida que o líquido chega ao separador. Este fenômeno tem potencial de causar grandes variações na planta de processamento (dificuldades na separação de óleo/água, distúrbios no sistema de compressão de gás) e até mesmo possíveis danos nos equipamentos.

Passo 4: Após a expulsão de grande parte do líquido e do bolsão de gás, o sistema apresentará uma velocidade de escoamento tão baixa que parte do líquido voltará para a base do riser, reiniciando o ciclo.

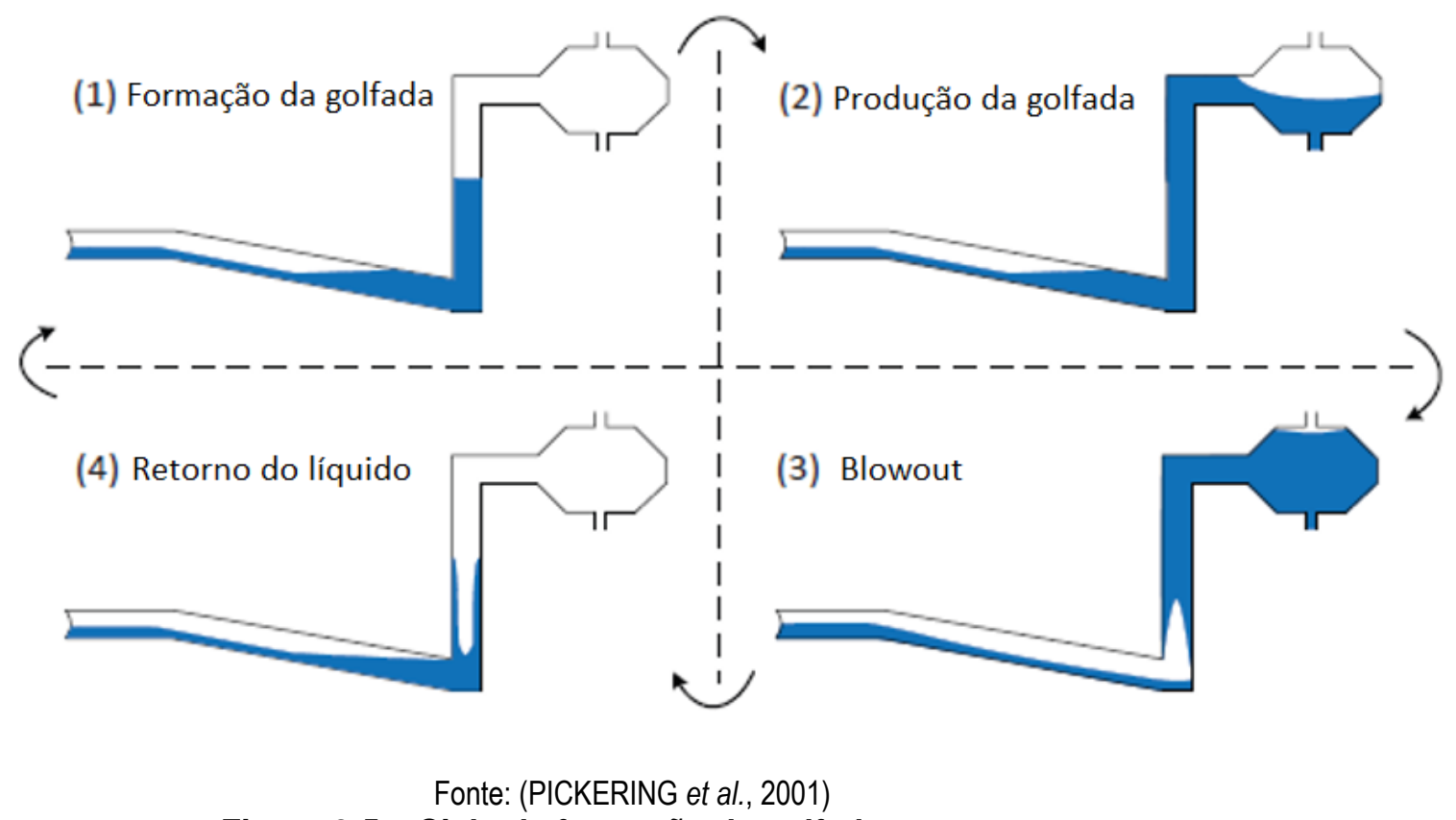

Figura 2-5 - Ciclo de formação de golfadas severas. 


\subsection{Estabilização de Escoamento em Golfadas}

Através do uso de técnicas de controle, é possível alterar as fronteiras de transição entre os regimes de escoamento e consequentemente estabilizar o poço, sem alterar a geometria dos dutos ou as condições de contorno originais do sistema (JAHANSHAHI, 2013).

Para avaliar o comportamento oscilatório de um sistema sujeito a golfadas, uma metodologia comumente empregada na literatura é a de levantar o diagrama de bifurcação. Tal diagrama ilustra o comportamento do sistema para todo o range de abertura da válvula choke. Na Figura 2-6, a linha contínua superior representa os valores máximos atingidos pela pressão de entrada e a linha inferior representa os valores mínimos desta pressão. Por último, a linha tracejada central representa a condição não oscilatória em regime permanente e a linha contínua central representa a pressão média entre os valores máximos e mínimos de pressão. Porém, sem um controle ativo, esta condição é instável (STORKAAS; SKOGESTAD, 2007).

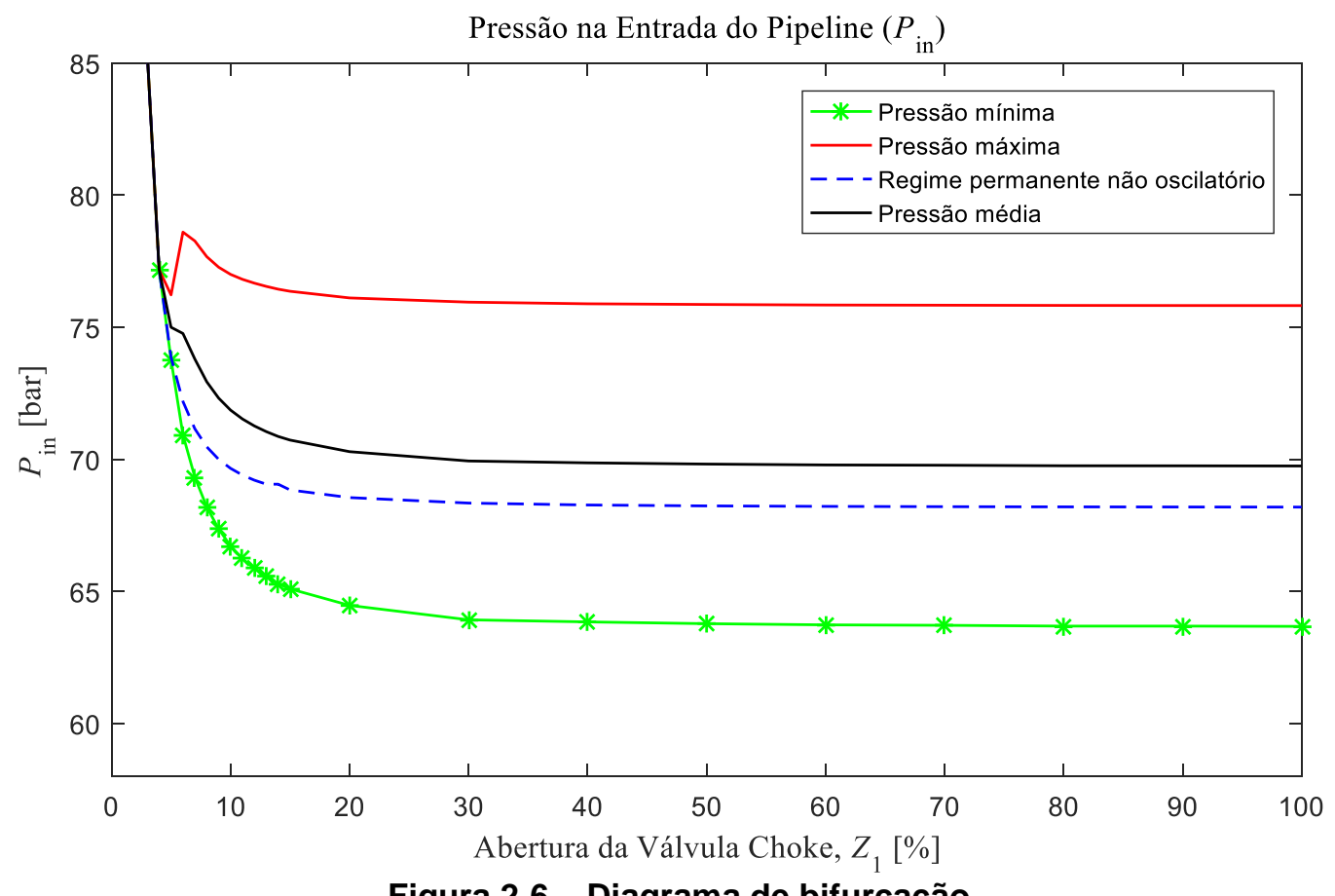

Figura 2-6 - Diagrama de bifurcação

Para o exemplo acima, o valor de abertura da válvula choke que representa a transição entre o escoamento estável (não oscilatório) e o escoamento com golfadas é de aproximadamente $5 \%$. É possível notar que, utilizando o controle ativo da válvula 
choke, a pressão média do sistema não oscilatório (linha tracejada central) é menor que o valor médio da pressão sem o controle da válvula (linha contínua central), sendo assim, operar na região instável, pode trazer ganhos de produtividade do poço através da menor contrapressão imposta ao reservatório (OGAZI et al., 2010).

Vários sistemas de controle têm sido desenvolvidos com o intuito de amenizar os problemas gerados pela alimentação intermitente nos sistemas de produção offshore. Uma metodologia empregada é utilizar um controle supervisório com chaveamento de controladores PID para o modo com golfadas e o modo sem golfadas, através da medida ou da estimativa da pressão da base (PEDERSEN; DURDEVIC; YANG, 2014). Em outra abordagem, utiliza-se de diferentes desempenhos de controladores PID para a válvula choke (CAMPOS et al., 2015). Demais aspectos de modelagem dinâmica voltada ao controle de golfadas são abordados em trabalhos de controle e observadores de estado (PEDERSEN; DURDEVIC; YANG, 2017), porém, o estado de arte não difere do que está descrito aqui no presente texto.

Outros trabalhos adotaram uma estratégia de controle em cascata com realimentação baseada em medidas de pressão de fundo e na vazão no topo do riser para suprimir as golfadas (FARD; GODHAVN; SAGATUN, 2006). Outros adotaram um sistema de controle PID que mede a pressão na base do riser e manipula a válvula choke para evitar golfadas no riser (STORKAAS; SKOGESTAD, 2007). Porém, a maior desvantagem destes sistemas é que os mesmos se baseiam em pressões que não estão localizados na superfície, ou seja, estes sensores estão sujeitos a falhas e possuem manutenção complexa e dispendiosa. 


\section{MATERIAIS E MÉTODOS}

\subsection{Controle Adaptativo}

Um sistema de controle adaptativo é um sistema não linear ainda que tanto o modelo da planta quanto o controlador sejam sistemas lineares. Ou seja, a adaptação dos parâmetros em malha fechada faz com que o sistema resultante se torne não linear (SILVA, 2014). Este controle é particularmente interessante para sistemas cujos parâmetros da planta são desconhecidos ou variam com o tempo.

Um sistema de controle adaptativo é gerado na tentativa de se controlar plantas com parâmetros desconhecidos através da variação automática dos parâmetros do controlador (NARENDRA; ANNASWAMY, 1989), (ASTOLFI; KARAGIANNIS; ORTEGA, 2010) como mostra a Figura 3-1.

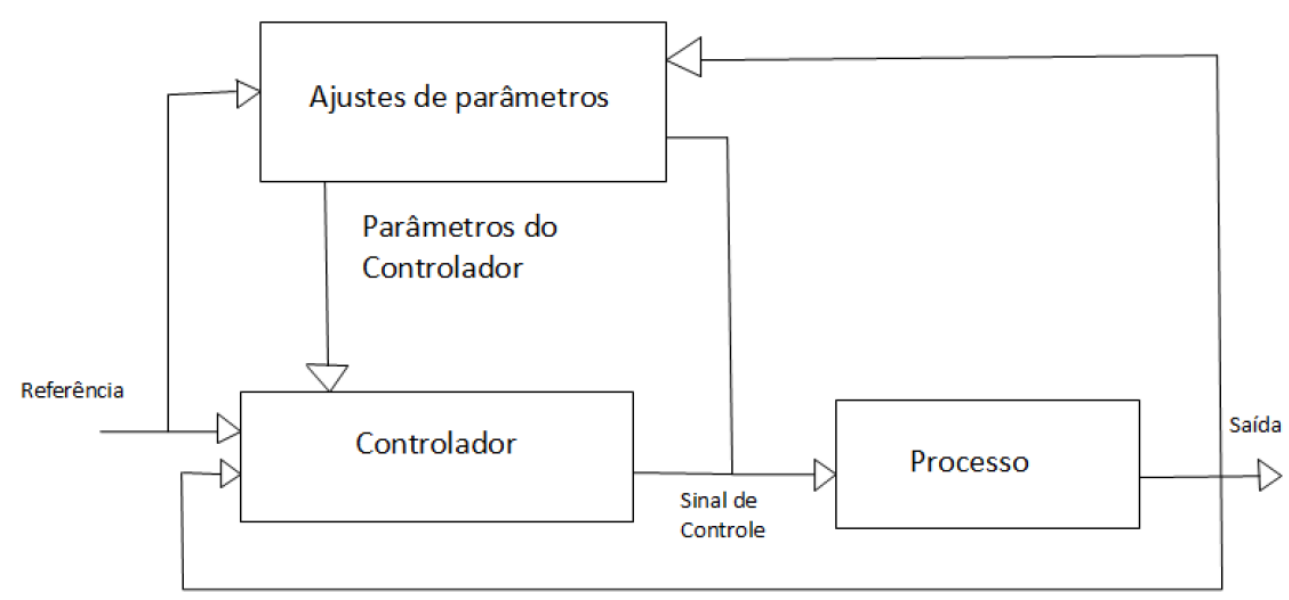

Fonte: (SILVA, 2014)

Figura 3-1 - Diagrama de blocos genérico de um controle adaptativo.

A construção de um controlador adaptativo contempla (ÅSTRÖM; WITTENMARK, 1995):

- A característica da resposta em malha fechada para o sistema

- Uma lei de controle com parâmetros ajustáveis

- Um mecanismo para o ajuste destes parâmetros

- A implementação da lei de controle 
Normalmente é necessário combinar técnicas de projeto de sistemas de controle com técnicas de estimação de parâmetros. Diversas estratégias de controle adaptativo são geradas através da combinação de cada uma das possíveis técnicas de estimação de parâmetros com cada uma das técnicas de projeto de controladores. Os esquemas de controladores adaptativos mais utilizados são (SILVA, 2014):

- Escalonamento de Ganho (Gain Scheduling)

- Controle Adaptativo por Modelo de Referência (Model Reference Adaptive Control)

- Regulador Auto-Sintonizável (Self-Tuning Regulator)

\subsubsection{Controle Adaptativo com Escalonamento de Ganho}

O Escalonamento de Ganho consiste em ajustar os parâmetros do controlador utilizando uma variável correlacionada, conforme mostrado na Figura 3-2.

Normalmente, varia-se os parâmetros do controlador de forma discreta dentro de um conjunto pré-definido de valores. A estratégia consiste em projetar controladores fixos para diferentes regiões de operação da planta e, em função das variáveis auxiliares, selecionar qual dos controladores é o mais adequado em um dado instante de tempo (LAGES, 2007).

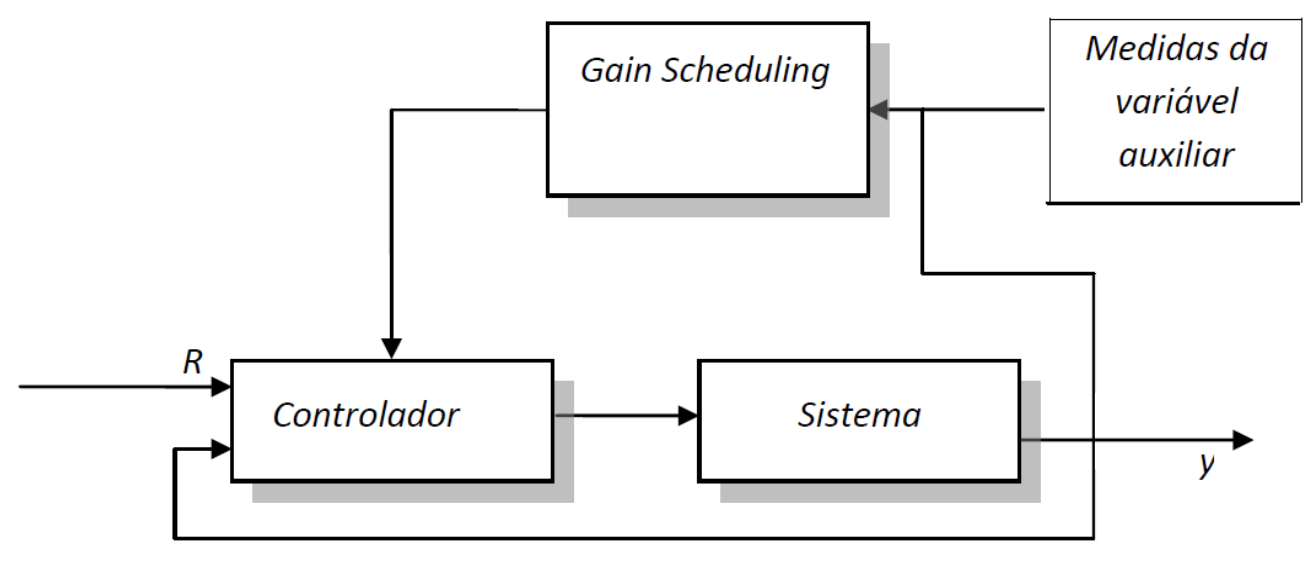

Fonte: (SILVA, 2014)

Figura 3-2 - Sistema de controle adaptativo por escalonamento de ganhos

Para a utilização do escalonamento de ganho é necessário conhecer a dinâmica de variação da planta em função de poucas variáveis facilmente mensuráveis. Apesar de 
ser um método bastante utilizado, há diversas desvantagens como: necessidade de alto esforço computacional, caso o número de regiões de operação da planta seja elevado, visto que é necessário projetar-se um controlador para cada uma destas regiões. Outra desvantagem consiste no fato de o escalonamento de ganho ser um mecanismo de adaptação em malha aberta. Uma dificuldade adicional inerente a este método é a prova de estabilidade do controlador resultante. Mesmo que se possa provar a estabilidade para todos os controladores associados a cada uma das regiões de operação, não há garantias de que, ao se chavear entre eles, o sistema resultante continuará estável (SILVA, 2014).

\subsubsection{Controle Adaptativo por Modelo de Referência}

O modelo de referência representa a dinâmica desejada para planta como mostra a Figura 3-3. O objetivo deste método de controle adaptativo é fazer com que a diferença entre a saída da planta e a saída do modelo de referência seja nula (LAGES, 2007).

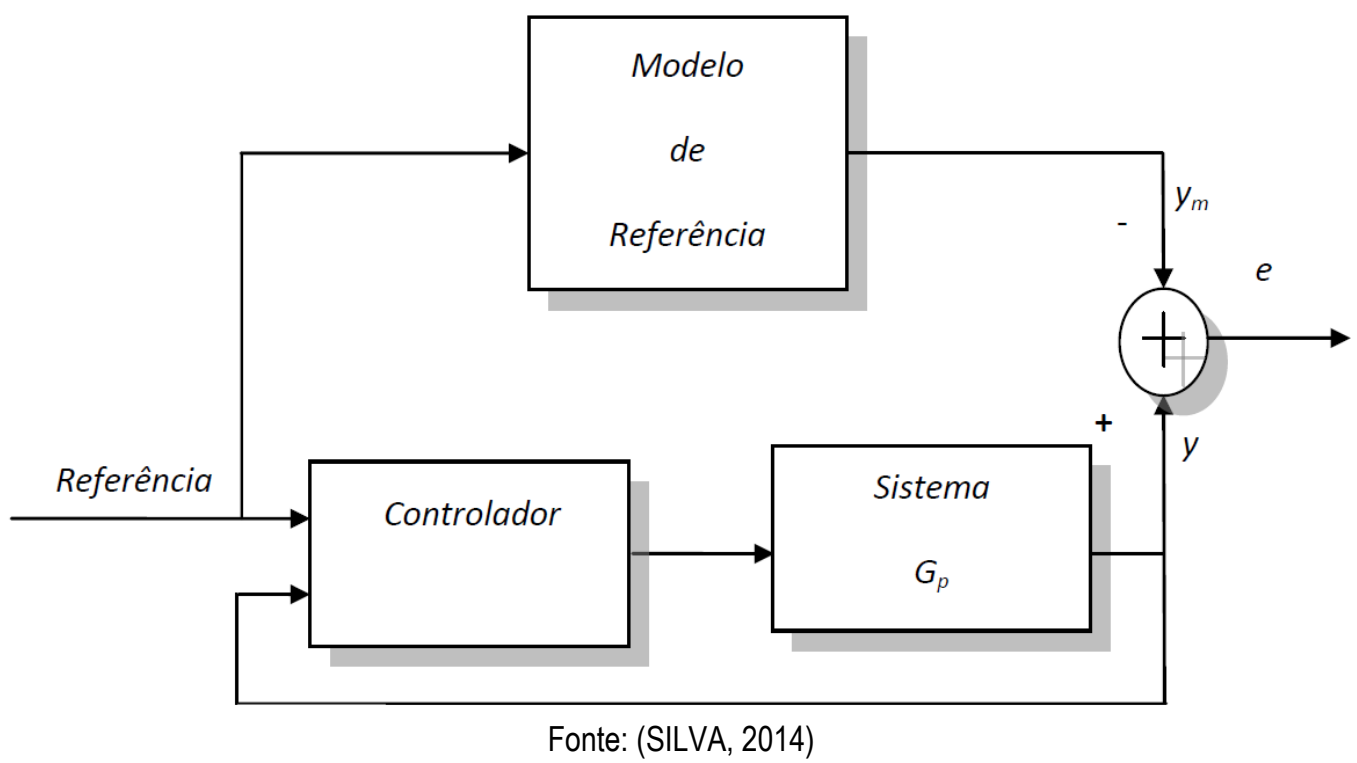

Figura 3-3 - Controle adaptativo por modelo de referência

Deve-se escolher o modelo de referência de forma a refletir as especificações de desempenho do sistema de controle, tais como: tempo de subida, tempo de acomodação, overshoot, etc. Porém, o modelo de referência deve apresentar comportamento possível de ser atingido pelo sistema de controle. Portanto, deve-se escolher um modelo de referência levando-se em conta as restrições em sua estrutura que são dependentes da estrutura do modelo de planta (SILVA, 2014). 
Utiliza-se um conjunto de parâmetros ajustáveis para parametrizar o controlador de forma a se obter a capacidade de rastreamento perfeito, logo, caso se conheça os parâmetros da planta, os parâmetros correspondentes do controlador devem ser tais que fazem a saída da planta convergir para a saída do modelo de referência. Para se provar a estabilidade e convergência, os métodos de controle adaptativo exigem que o controlador possa ser parametrizado linearmente, ou seja, que a lei de controle seja linear com relação aos seus parâmetros (LAGES, 2007). O problema é obter um mecanismo de ajuste dos parâmetros do controlador que faça com que o erro de saída $e=y-y m$ passe a convergir para zero. Nas primeiras versões do controlador, era utilizado um mecanismo de adaptação baseado no gradiente. Supondo que $\Psi$ seja o vetor de parâmetros do controlador, a ideia é reduzir $e^{2}(\Psi)$ através do ajuste de $\Psi$, de forma a causar a sua maior redução, ou seja:

$$
\frac{d \Psi}{d t}=-K \frac{\partial}{\partial \Psi}\left(e^{2}(\Psi)\right)=-2 K e(\Psi) \frac{\partial}{\partial \Psi}(e(\Psi))
$$

onde $K$ é uma constante positiva denominada ganho de adaptação.

A variável $e(\Psi)$ é o erro de saída obtido supondo-se que os parâmetros do controlador são $\Psi$. Como o gradiente de $e(\Psi)$ é igual ao gradiente de $y(\Psi)$, já que $y_{m}$ é independente de $\Psi$, pode-se escrever:

$$
\frac{d \Psi}{d t}=-2 K e(\Psi) \frac{\partial}{\partial \Psi}(y(\Psi))
$$

A função de sensitividade $\frac{d y(\Psi)}{d \Psi}$ usualmente depende dos parâmetros da planta, que são desconhecidos. Para resolver este problema foi proposta uma regra que consiste em utilizar as estimativas no instante $t$ ao invés de parâmetros desconhecidos (SILVA, 2014). Em geral não é possível obter-se os zeros (SASTRY; BODSON, 1989) para os esquemas baseados nesta regra, no entanto quando o ganho de adaptação e a amplitude da entrada referência são pequenos, o esquema apresenta bom desempenho (MARRELS et al., 1986).

A substituição da função de sensitividade $\frac{d y(\Psi)}{d \Psi}$ por outras funções, permite que se obtenha a prova de estabilidade e propriedades de convergência através da teoria de Lyapunov (NARENDRA; LIN; VALAVANI, 1980). 


\subsubsection{Controle Adaptativo Auto-Sintonizado}

No controle adaptativo auto-sintonizado altera-se os parâmetros do controlador a cada mudança dos parâmetros da planta (ÅSTRÖM; WITTENMARK, 1989) como mostrado na Figura 3-4. O controlador possui basicamente duas etapas: a estimação dos parâmetros e o projeto do controlador.

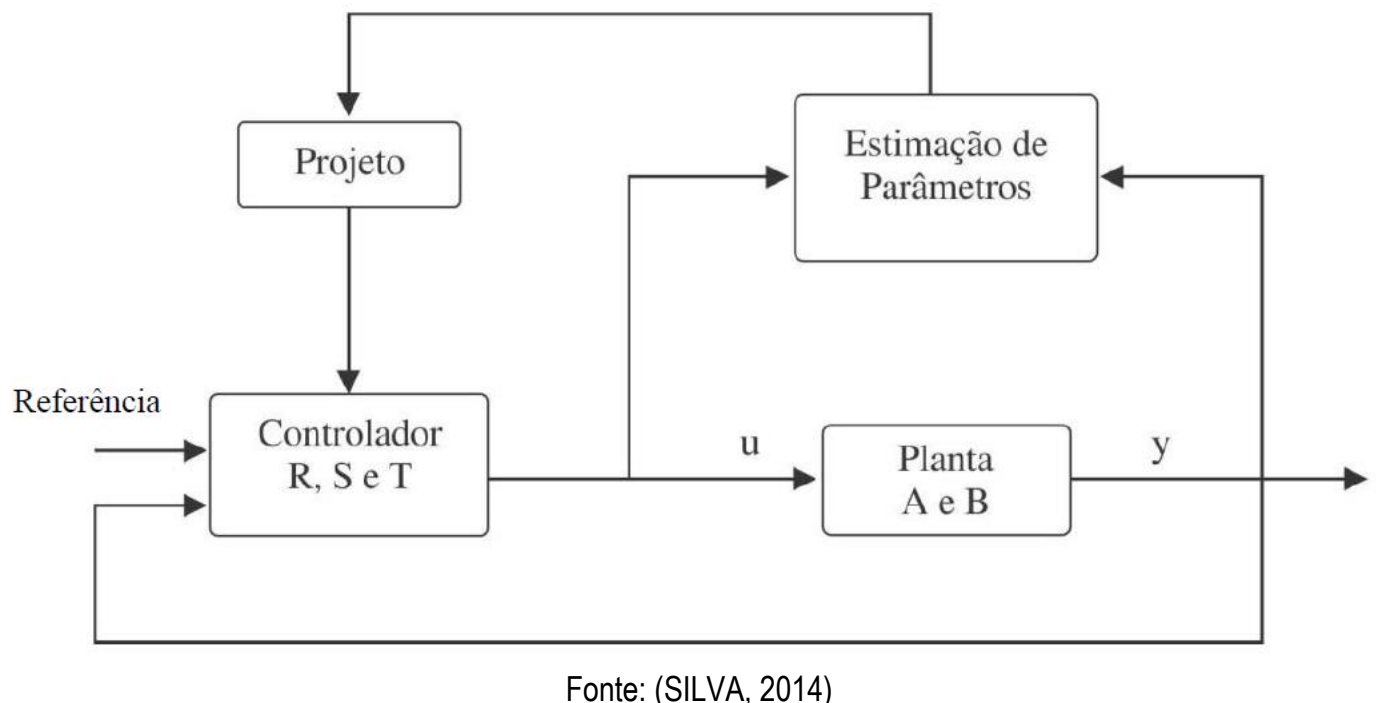

Figura 3-4 - Controle adaptativo auto-sintonizado

- Estimação de parâmetros: Nesta etapa, realiza-se a identificação da planta, obtendo sua função de transferência, que posteriormente é utilizada no projeto do controlador. A estimação de parâmetros é realizada utilizando métodos de estimação recursivos, ou seja, a cada recursão os parâmetros são atualizados com base na última medição e nos valores anteriores. Há vários métodos para estimar on-line os parâmetros do controlador, tais como: aproximação estocástica, mínimos quadrados, mínimos quadrados estendido generalizados, variáveis instrumentais e máxima verossimilhança, sendo estes parâmetros atualizados e ajustados a cada período de amostragem para obter-se o controle desejado do sistema (SILVA, 2014).

- Projeto do controlador: Nesta etapa calcula-se os parâmetros do controlador de forma a garantir um bom desempenho sem erro estacionário considerando um modelo de referência. O projeto é realizado em tempo real e a solução apresentada refere-se ao sistema cujos parâmetros foram estimados na etapa 
anterior. Existem diversos métodos numéricos para o projeto do controlador, tais como: mínima variância, linear quadrática, alocação de polos, modelo de referência e auto sintonizável (SILVA, 2014).

Caso os parâmetros da planta sejam desconhecidos, substitui-se os parâmetros, pelas suas estimativas determinadas por algum estimador on-line de parâmetros. $\mathrm{O}$ controlador obtido combinando-se um esquema de identificação on-line de parâmetros com um controlador convencional, é denominado Self-Tuning Regulator (ÅSTRÖM; WITTENMARK, 1973).

Tipicamente, desenvolve-se os controladores auto-sintonizados em tempo discreto. A cada instante de amostragem os parâmetros da planta $\theta$ são identificados com base nas entradas $u$ e saídas $y$ anteriores. Os parâmetros do controlador $\psi$ são determinados e o sinal de controle $u$ é gerado. Nota-se que, nos controladores autosintonizados, os parâmetros do controlador são determinados de forma a ajustar-se aos dados de entrada e saída da planta, enquanto nos controladores por modelo de referência, os parâmetros são ajustados de forma a fazer o erro de trajetória convergir para zero. Pode ser mostrado (ÅSTRÖM; WITTENMARK, 1989), que a abordagem dos controladores por modelo de referência é um caso particular do controladores auto-sintonizados, utilizando-se de uma parametrização adequada.

É mais complexo garantir a estabilidade e a convergência de um controlador autosintonizado que no controlador por modelo de referência, que normalmente exige suposição sobre as propriedades dos sinais envolvidos. Os controladores autosintonizados são obtidos combinando-se uma técnica de estimação de parâmetros com uma técnica de controle. As técnicas de controle mais utilizadas são: Variância Mínima, Variância Mínima Generalizada, Controle Preditivo Generalizado, Alocação de polos e controle Linear Quadrático Gaussiano. Para possibilitar a implementação do controlador em tempo real, utilizam-se técnicas de estimação dos parâmetros recursivas, principalmente: Mínimos Quadrados Recursivo e Mínimos Quadrados Estendido (SILVA, 2014). 


\subsection{Regulador Linear Quadrático (LQR)}

O regulador linear quadrático (LQR) é um controlador ótimo que foi amplamente difundido na literatura em diversas aplicações (SUN; GAN, 2010) e (UYGUN; MATTHEW; HUANG, 2006). O regulador linear quadrático possui características excelentes de desempenho e é de simples implementação. Este tipo de estratégia de controle inclui a realimentação dos estados, que são ponderados de forma a minimizar uma função custo (BURNS, 2001).

No controle utilizando regulador linear quadrático, assume-se que a dinâmica da planta é linear e conhecida.

$$
\begin{gathered}
x_{k+1}=A_{d} x_{k}+B_{d} u_{k} \\
y_{k}=C_{d} x_{k}
\end{gathered}
$$

Dado um sistema da Equação 3.3 com um estado inicial $x(0)$, encontra-se o sinal de entrada ótimo $u_{k}$, que leva o sistema para o estado zero $x=0$ de maneira ótima, através da minimização da função custo:

$$
J=\frac{1}{2} \sum_{k=0}^{\infty} x_{k}^{T} Q x_{k}+u_{k}^{T} R u_{k}
$$

Isto requer que o par $\left(A_{d}, B_{d}\right)$ seja estabilizável, ou seja, que seus estados possam ser transferidos para a origem de qualquer estado inicial em um tempo infinito (SYRE, 2012). $Q$ e $R$ são matrizes simétricas positivas definidas para os estados $x$ e a entrada $u$ respectivamente. O objetivo é minimizar a função custo, então se $R$ é alto, utilizar a entrada $u$ será caro e fornececerá uma resposta lenta. Tendo um alto valor no primeiro elemento de $Q$ comparada aos outros elementos da diagonal, o controlador irá priorizar o primeiro estado. Adicionalmente, se $Q$ é muito maior que $R$, obtém-se uma resposta rápida. O ótimo entre $Q$ e $R$ muitas vezes é encontrado por tentativa e erro.

A solução ótima é $u_{k}=-K_{l q r} x_{k}$, onde:

$$
K_{l q r}=R^{-1} B_{d}{ }^{T} P
$$

E $P$ é a solução definida simétrica positiva da equação de Riccatti.

$$
A_{d}{ }^{T} P+P A_{d}-P B_{d} R^{-1} B_{d}{ }^{T} P+Q=0
$$


É possível estabelecer um compromisso entre a amplitude das variáveis de estado, a velocidade de resposta do sistema e a amplitude do sinal de controle, através da escolha adequada dos índices de desempenho relativos a estas variáveis. Porém, a utilização deste tipo de estratégia de controle é comprometida na presença de distúrbios e/ou quando há variações paramétricas no sistema a ser controlado. Isto ocorre pois a lei de controle depende diretamente dos estados da planta, ou seja, caso existam dinâmicas não consideradas, a ação de controle é prejudicada por não conter todas as informações referentes ao sistema (KANIESKI, 2010).

\subsection{Observadores de Estado}

Em projetos de sistemas de controle considera-se que todas as variáveis de estado sejam acessíveis para medição, porém, na prática isto pode não ser verdade. Logo, é necessário estimar estas variáveis não mensuráveis ou não medidas. Este processo de estimação das variáveis de estado é comumente chamado de observação. Quando um observador de estado estima todas as variáveis de estado do sistema, mesmo que algumas delas sejam mensuráveis, é denominado observador de ordem plena. Se apenas algumas das variáveis de estado do sistema são acessíveis, pode-se desenvolver um observador de estado que estima somente aquelas inacessíveis para medição. Neste caso o observador é denominado observador de estado de ordem reduzida ou observador de estado de ordem mínima (OLIVEIRA, 2014), sendo a planta definida por:

$$
\begin{array}{r}
\dot{x}=A x+B u \\
y=C x
\end{array}
$$

Nestas equações 3.6 e 3.7, por exemplo, $x$ é o vetor de estado de dimensão $n$, i.e., $\left(x \in \mathbb{R}^{n}\right)$, $u$ é o vetor de controle de dimensão $r$, i.e., $\left(u \in \mathbb{R}^{r}\right), y$ é o vetor de resposta ou saída de dimensão $m$, i.e., $\left(y \in \mathbb{R}^{m}\right)$, $A$ é a matriz $n \times n$ de estado $\left(A \in \mathbb{R}^{n \times n}\right)$, $B$ é a matriz $n \times r$ de controle ou entrada $\left(B \in \mathbb{R}^{n \times r}\right)$, e $C$ é a matriz $m \times n$ de saída ou de observação $\left(C \in \mathbb{R}^{m \times n}\right)$. Por concisão, são omitidas as especificações das dimensões das matrizes quando não houver necessidade. 
O modelo matemático é o mesmo da planta, exceto por incorporar um termo adicional que compensa as incertezas nas matrizes $A$ e $B$ e a ausência do erro inicial visto que o observador é um subsistema reconstrutor do vetor de estado da planta. $O$ erro de estimação ou erro de observação pode ser definidos pela diferença entre a saída medida e a saída estimada. O erro inicial é a diferença entre o estado inicial e o estado inicial estimado (OLIVEIRA, 2014). Logo, define-se o modelo matemático de um observador como:

$$
\dot{\tilde{x}}=A \tilde{x}+B u+K_{e}(y-C \tilde{x})=\left(A-K_{e} C\right) \tilde{x}+B u+K_{e} y
$$

Onde $\tilde{x}$ é o estado estimado e $C \tilde{x}$ é a saída estimada. As entradas do observador são a saída $y$ e a entrada $u$. A matriz $K_{e}$ é denominada matriz de ganho do observador que atua como um termo de correção que envolve a diferença entre a saída medida $y$ e a saída estimada $C \tilde{x}$. Esse termo corrige continuamente a saída do modelo e aumenta o desempenho do observador. A Figura 3-5 mostra o diagrama de blocos do sistema e do observador de estado de ordem plena.

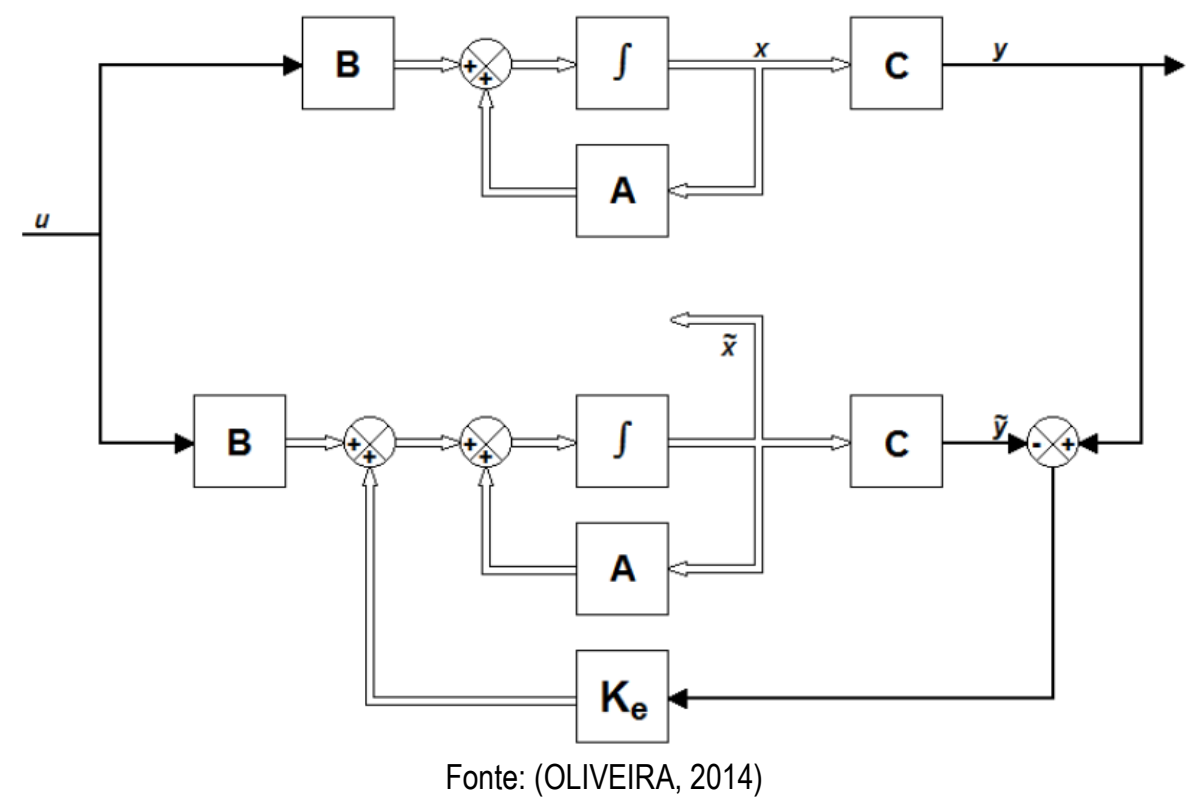

Figura 3-5 - Observador de estado de ordem plena

\subsubsection{Observador de Estado de Ordem Plena}

Conforme citado anteriormente, o observador de ordem plena consiste em um observador com a mesma ordem da planta, mesmo que todos seus estados sejam mensuráveis. 
Supondo que a planta seja definida pelas equações 3.6 e 3.7 , tem-se que para encontrar o erro de observação, subtrai-se a equação 3.8 a partir da equação 3.6 :

$$
\dot{x}-\dot{\tilde{x}}=A x-A \tilde{x}-K_{e}(C x-C \tilde{x})=\left(A-K_{e} C\right)(x-\tilde{x})
$$

Definindo o vetor de erro como $e=x-\tilde{x}$, a Equação 3.9 se torna:

$$
\dot{e}=\left(A-K_{e} C\right) e
$$

Nota-se na Equação 3.10, que o comportamento dinâmico do vetor de erro é determinado pelos autovalores da matriz $\left(A-K_{e} C\right)$. Se a matriz $\left(A-K_{e} C\right)$ for uma matriz estável, o vetor de erro convergirá para zero, qualquer que seja o vetor de erro inicial $e(0)$. Ou seja, $\tilde{x}(t)$ convergirá para $x(t)$ independentemente do valor de $x(0) \mathrm{e}$ $\tilde{x}(0)$. Se os autovalores da matriz $\left(A-K_{e} C\right)$ forem escolhidos de tal maneira que 0 comportamento do vetor de erro seja assintoticamente estável e adequadamente rápido, então qualquer vetor de erro tenderá a zero com uma velocidade adequada. Se a planta for completamente observável, é possível escolher a matriz $K_{e}$ tal que $\left(A-K_{e} C\right)$ tenha seus autovalores escolhidos arbitrariamente. Para tanto, é preciso verificar a condição necessária e suficiente para observação de estado. Ou seja, verifica-se que o posto da matriz abaixo seja $n$ :

$$
\left[\begin{array}{c}
C^{*} \\
C^{*} A^{*} \\
\vdots \\
C^{*} A^{* n-1}
\end{array}\right]
$$

A condição de observabilidade completa do sistema original é definida pelas Equações 3.6 e 3.7. Uma vez selecionado os autovalores desejados, o observador de estado de ordem plena poderá ser projetado, desde que a planta seja completamente observável. Os autovalores desejados da equação característica devem ser escolhidos de modo que o observador responda, pelos menos, duas a cinco vezes mais rápido que o sistema em malha fechada considerado. $O$ processo de projeto, portanto, passa a ter dois estágios, sendo o primeiro a determinação da matriz $K$ de realimentação, que produzirá a equação característica desejada, e o segundo consiste na determinação da matriz de ganho do observador que produzirá a equação do observador desejada (OLIVEIRA, 2014). A Figura 3-6 apresenta o diagrama de blocos de um sistema de controle realimentado por estado observado. 


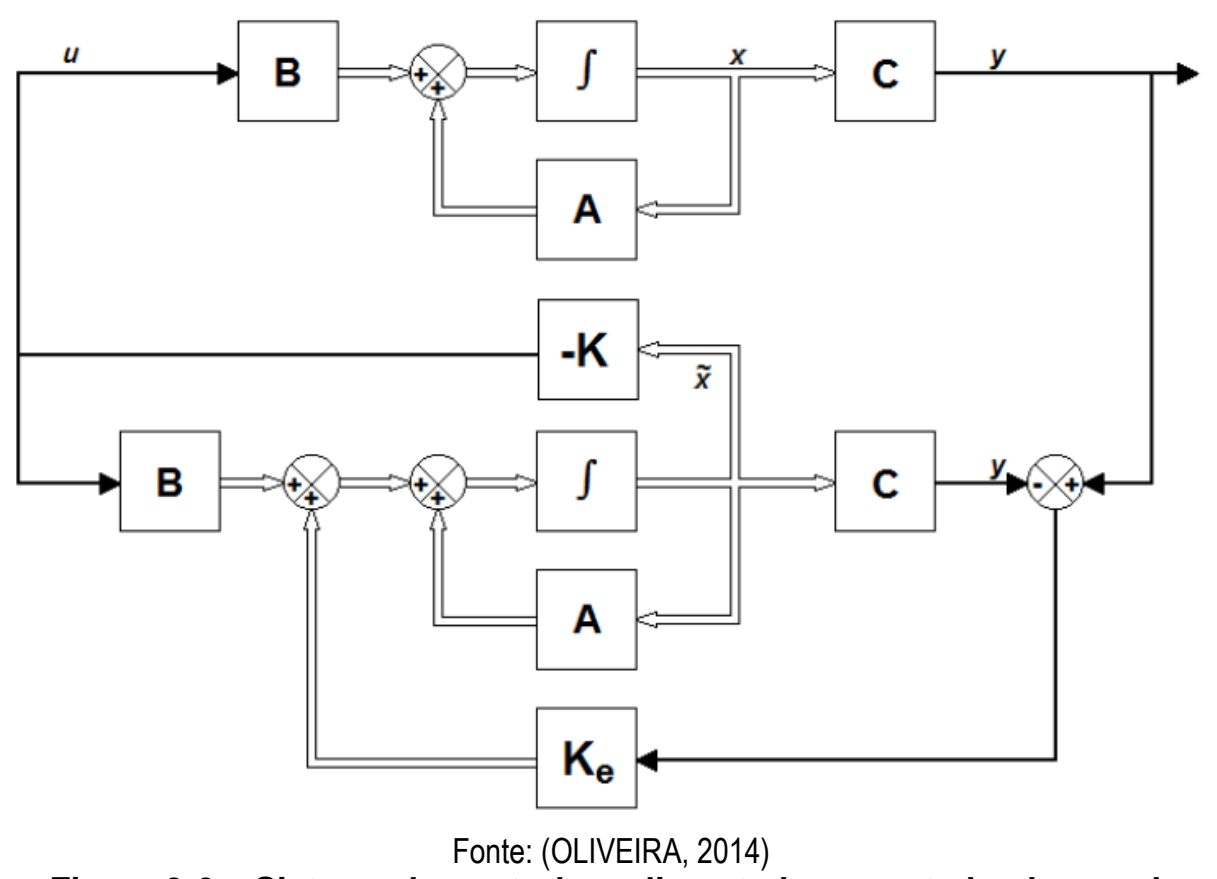

Figura 3-6 - Sistema de controle realimentado por estado observado

Considerando um sistema completamente controlável e observável, tem-se a lei de controle baseado no estado observado $\tilde{x}$ :

$$
u=-K \tilde{x}
$$

Que produz a equação de estado:

$$
\dot{x}=A x-B K \tilde{x}=(A-B K) x+B K(x-\tilde{x})
$$

Como o erro $e(t)$ é dado por $x(t)-\tilde{x}(t)$, reescreve-se a equação 3.13 como:

$$
\dot{x}=(A-B K) x+B K e
$$

Combinando a equação 3.10 do erro do observador com a equação 3.14, tem-se:

$$
\left[\begin{array}{c}
\dot{x} \\
\dot{e}
\end{array}\right]=\left[\begin{array}{cc}
A-B K & B K \\
0 & A-K_{e} C
\end{array}\right]\left[\begin{array}{l}
x \\
e
\end{array}\right]
$$

A equação 3.15 descreve as dinâmicas do sistema de controle realimentado por estado observado. A equação característica desse sistema é:

$$
\left[\begin{array}{cc}
s I-A+B K & -B K \\
0 & s I-A+K_{e} C
\end{array}\right]=0
$$

ou

$$
|s I-A+B K|\left|s I-A+K_{e} C\right|=0
$$


Os polos de malha fechada do sistema de controle realimentado por estado observado consistem nos polos do projeto por alocação de polos e nos polos do projeto isolado do observador. Ou seja, o projeto da alocação de polos e o projeto do observador são independentes entre si. Eles podem ser conduzidos separadamente e combinados para formar o sistema de controle realimentado por estado observado (OLIVEIRA, 2014).

Supondo que a planta seja completamente observável, ao se aplicar uma lei de controle por realimentação de estado observado, tem-se que as equações do observador serão dadas por:

$$
\begin{gathered}
\dot{\tilde{x}}=\left(A-K_{e} C-B K\right) \tilde{x}+K_{e} y \\
u=-K \tilde{x}
\end{gathered}
$$

Supondo uma condição inicial nula e resolvendo a transformada de Laplace para $\tilde{X}(S)$ da Equação 3.18, tem-se:

$$
\tilde{X}(S)=\left(s I-A+K_{e} C+B K\right)^{-1} K_{e} Y(S)
$$

Substituindo $\tilde{X}(S)$ na Equação 3.19 e resolvendo a transformada de Laplace, obtémse a seguinte função de transferência:

$$
\begin{gathered}
U(S)=-K\left(s I-A+K_{e} C+B K\right)^{-1} K_{e} Y(S) \\
\frac{U(S)}{Y(S)}=-K\left(s I-A+K_{e} C+B K\right)^{-1} K_{e}
\end{gathered}
$$

A Figura 3-7 mostra a representação em diagramas de blocos do sistema. Nota-se que a função de transferência $K\left(s I-A+K_{e} C+B K\right)^{-1} K_{e}$ atua como controlador do sistema. Por isso, denomina-se esta função de transferência como função de transferência do controlador-observador. 


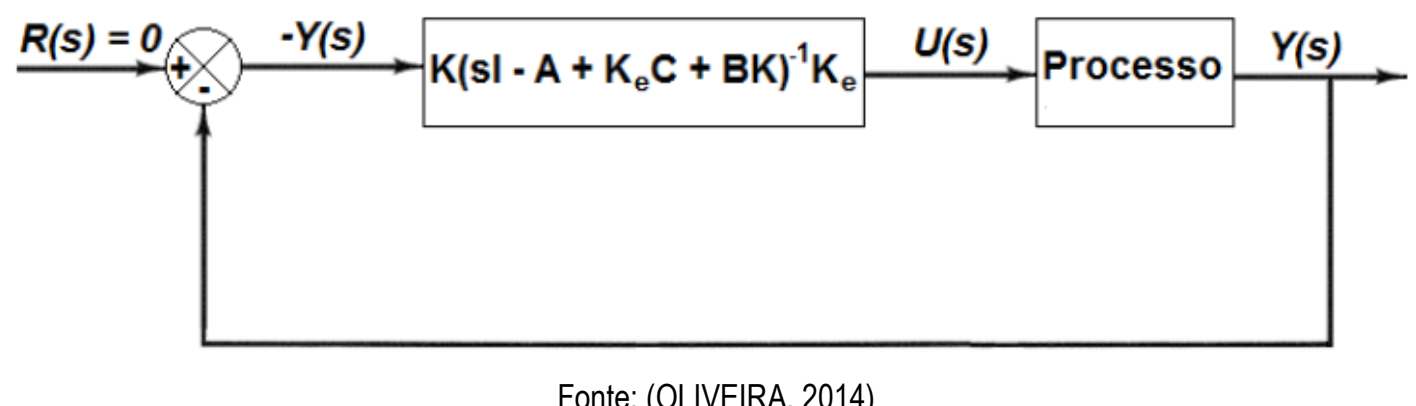

Figura 3-7 - Diagrama de blocos do sistema com controlador-observador

A matriz do controlador-observador $A-K_{e} C-B K$ pode ser estável ou não, embora $A-B K$ e $A-K_{e} C$ sejam escolhidas para serem estáveis.

\subsubsection{Observador de Ordem Reduzida}

Algumas variáveis de estado podem ser medidas com exatidão, logo, não necessitam ser estimadas ou observadas. Suponha que um vetor de estado $x$ seja um vetor de dimensão $n$ e que a saída seja um vetor $y$ de dimensão $m$ que pode ser medido. Como as variáveis $m$ de saída são combinações lineares das variáveis de estado, então $m$ variáveis de estado não precisam ser estimadas. É necessário estimar apenas $n-m$ variáveis de estado. Então, um observador de ordem reduzida se torna um observador de ordem $(n-m)$, que também pode ser denominado como um observador de ordem mínima (OLIVEIRA, 2014). A Figura 3-8 mostra um diagrama de blocos de um sistema com um observador de ordem mínima.

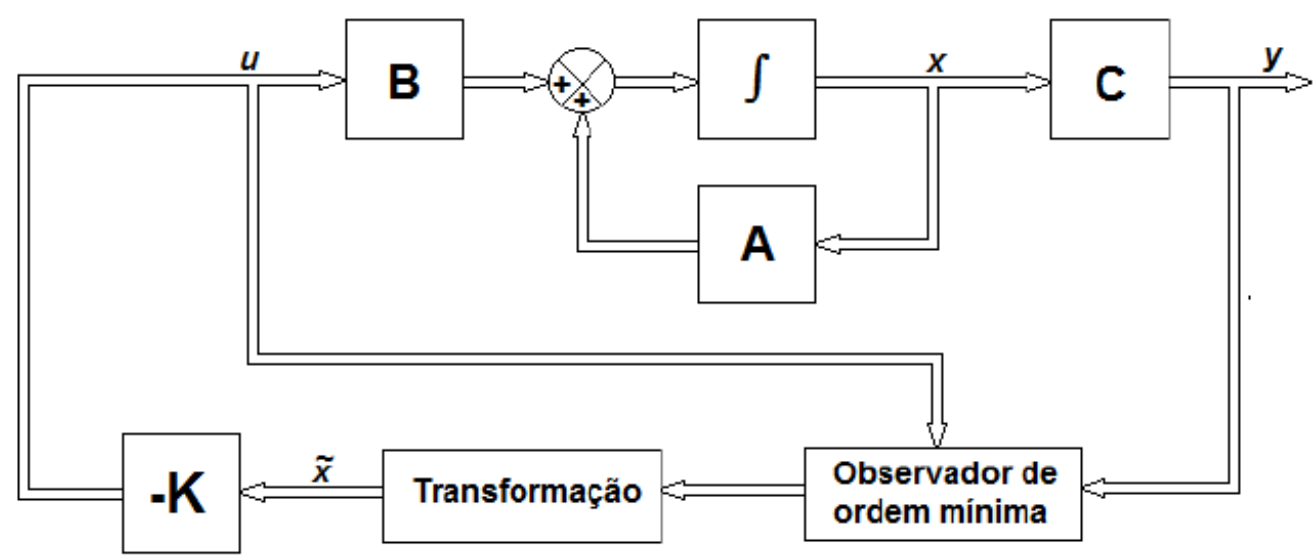

Fonte: (OLIVEIRA, 2014)

Figura 3-8 - Sistema de controle realimentado por estado observado de ordem mínima 
A ideia básica do observador de ordem mínima, é que a saída que pode ser medida seja um escalar (ou seja $m=1$ ). Considera-se então, o sistema como descrito pelas Equações 3.6 e 3.7, onde o vetor $x$ é particionado em duas partes: $x_{a}$ (escalar) que é igual a saída $y \mathrm{e}$, portanto, pode ser medida diretamente e $x_{b}$ (vetor com dimensão $n-1)$, que é a porção não medida do vetor de estado. Desse modo, a equação de estado particionada e a de saída resultam em:

$$
\begin{gathered}
{\left[\begin{array}{c}
\dot{x_{a}} \\
\cdots \\
\dot{x_{b}}
\end{array}\right]=\left[\begin{array}{ccc}
A_{a a} & \vdots & A_{a b} \\
\cdots & \cdots & \cdots \\
A_{b a} & \vdots & A_{b b}
\end{array}\right]\left[\begin{array}{c}
x_{a} \\
\cdots \\
x_{b}
\end{array}\right]+\left[\begin{array}{c}
B_{a} \\
\cdots \\
B_{b}
\end{array}\right] u} \\
y=\left[\begin{array}{lll}
1 & \vdots & 0
\end{array}\right]\left[\begin{array}{c}
x_{a} \\
\cdots \\
x_{b}
\end{array}\right]
\end{gathered}
$$

onde $A_{a a}$ é um escalar, $\mathrm{A}_{\mathrm{ab}}$ é uma matriz $1 \times(n-1), \mathrm{A}_{\mathrm{ba}}$ é uma matriz $(n-1) \times 1, \mathrm{~A}_{\mathrm{bb}}$ é uma matriz $(n-1) \times(n-1), \mathrm{B}_{\mathrm{a}}$ é uma matriz $L \times r$, e $\mathrm{B}_{\mathrm{b}}$ é uma matriz $(n-1) \times r$.

A partir da Equação 3.23, pode-se retirar a equação da porção mensurável do estado que resulta em:

$$
\dot{x_{a}}=A_{a a} x_{a}+A_{a b} x_{b}+B_{a} u
$$

ou

$$
\dot{x_{a}}-A_{a a} x_{a}-B_{a} u=A_{a b} x_{b}
$$

As equações 3.25 e 3.26 agem como equações de saída. Considera-se o lado esquerdo das equações 3.25 e 3.26 como quantidades conhecidas para o projeto de observadores de ordem mínima. Da mesma forma, a partir da equação 3.23, pode-se retirar a equação não mensurável do estado que resulta em:

$$
\dot{x_{b}}=A_{b a} x_{a}+A_{b b} x_{b}+B_{b} u
$$

Como as quantidades $A_{b a} x_{a}$ e $B_{b} u$ são conhecidas, a Equação 3.27 descreve as dinâmicas da porção não mensurável do estado.

É possível comparar a equação de estado do observador de ordem plena obtido a partir de $\dot{x}=A x+B u$, com a equação de estado do observador de ordem mínima dada pela Equação 3.27. Da mesma forma, é possível comparar as equações de saída 
do observador de ordem plena dada por $y=C x$ com a equação de saída do observador de ordem mínima dada pelas Equações 3.25 e 3.26.

O projeto de observadores de ordem mínima pode ser construído a partir do observador de ordem plena substituindo as quantidades de $u=-K \tilde{x}$, pelas quantidades apresentadas nas equações 3.25, 3.26 e 3.27, associando cada quantidade com o valor comparado para ordem plena. A Tabela 1 lista as substituições necessárias para escrever a equação do observador de ordem mínima.

Tabela 1 - Substituições para a equação do observador de ordem reduzida

\begin{tabular}{|c|c|}
\hline $\begin{array}{c}\text { Observador de estado de } \\
\text { ordem plena }\end{array}$ & $\begin{array}{c}\text { Observador de estado de } \\
\text { ordem mínima }\end{array}$ \\
\hline$\tilde{x}$ & $\widetilde{x_{b}}$ \\
\hline$A$ & $A_{b b}$ \\
\hline$B u$ & $A_{b a} x_{a}+B_{b} u$ \\
\hline$y$ & $\dot{x}_{a}-A_{a a} x_{a}-B_{a} u$ \\
\hline$C$ & $A_{a b}$ \\
\hline$K_{e}($ matriz n x 1) & $K_{e}($ matriz $(\mathrm{n}-1) \times 1)$ \\
\hline
\end{tabular}

Fonte: (OLIVEIRA, 2014)

Fazendo as substituições necessárias, constrói-se a equação do observador de ordem mínima como segue:

$$
\dot{\widetilde{x_{b}}}=\left(A_{b b}-K_{e} A_{a b}\right) \tilde{x}_{b}+A_{b a} x_{a}+B_{b} u+K_{e}\left(\dot{x}_{a}-A_{a a} x_{a}-B_{a} u\right)
$$

onde a matriz $K_{e}$ do observador de estado é uma matriz $(n-1) \times 1$.

Na Equação 3.28, observa-se que para estimar $x_{b}$, é necessário diferenciar $x_{a}$, o que representa uma dificuldade, pois a diferenciação amplifica ruídos. Portanto, analisando as equações 3.25 e 3.26, pode-se reescrever a equação do observador de ordem mínima como:

$$
\dot{\widetilde{x_{b}}}=\left(A_{b b}-K_{e} A_{a b}\right) \tilde{x}_{b}+A_{b a} x_{a}+B_{b} u+K_{e} A_{a b} x_{b}
$$


Em seguida, obtém-se a equação do erro do observador de ordem mínima. Então, subtraindo a Equação 3.29 da Equação 3.27, tem-se:

$$
\dot{x}_{b}-\dot{x_{b}}=\left(A_{b b}-K_{e} A_{a b}\right)\left(x_{b}-\tilde{x}_{b}\right)
$$

Por definição, tem-se que $e=x_{b}-\tilde{x}_{b}$, o que transforma a Equação 3.30 em:

$$
\dot{e}=\left(A_{b b}-K_{e} A_{a b}\right) e
$$

A partir da Equação 3.31, pode-se obter as dinâmicas de erro seguindo a mesma técnica desenvolvida para o observador de ordem plena. Para isso, tem-se que o posto seja $(n-1)$ na seguinte matriz:

$$
\left[\begin{array}{c}
A_{a b} \\
A_{a b} A_{b b} \\
\vdots \\
A_{a b} A_{b b}^{n-2}
\end{array}\right]
$$

Esta é a condição de observabilidade completa aplicada ao observador de ordem mínima. A equação característica do observador de ordem mínima é obtida a partir da Equação 3.31, como segue:

$$
\begin{gathered}
\left|s I-A_{b b}+K_{e} A_{a b}\right|=\left(s-\mu_{1}\right)\left(s-\mu_{2}\right) \cdots\left(s-\mu_{n-1}\right)=s^{n-1}+\hat{\alpha}_{1} s^{n-2}+\cdots+ \\
\hat{\alpha}_{n-2} s+\hat{\alpha}_{n-1}=0
\end{gathered}
$$

onde $\mu_{1}, \mu_{2}, \cdots, \mu_{n-1}$ são os autovalores desejados do observador de ordem mínima. $\mathrm{A}$ matriz de ganho $K_{e}$ do observador pode ser determinada escolhendo-se primeiro os autovalores desejados do observador de ordem mínima e utilizando-se do procedimento já desenvolvido para o observador de ordem plena com as modificações apropriadas.

Para o sistema de controle realimentado por estado observado com observador de ordem plena, tem-se que os polos de malha fechada consistem nos polos devido ao projeto isolado da alocação de polos e dos polos devidos ao projeto isolado do observador. Para o caso do sistema de controle realimentado por estado observado com observador de ordem mínima, a mesma conclusão se aplica. Portanto, a equação característica pode ser obtida como:

$$
|s I-A+B K|\left|s I-A_{b b}+K_{e} A_{a b}\right|=0
$$


Os polos de malha fechada do sistema de controle realimentado por estado observado com um observador de ordem mínima compreendem os polos de malha fechada da alocação de polos, ou seja, autovalores da matriz $(A-B K)$, e os polos de malha fechada devido ao observador de ordem mínima, ou seja, autovalores da matriz $\left(A_{b b}\right.$ $\left.K_{e} A_{a b}\right)$. Portanto, o projeto da alocação de polos e o projeto do observador de estado de ordem mínima são independentes entre si.

\subsection{Filtro de Kalman}

\subsubsection{Filtro de Kalman Estendido (EKF)}

Em alguns casos, as saídas do sistema são corrompidas por sinais de ruído causados por motivos diversos (por ex. ruídos elétricos gerados por transientes de energia ou ruídos captados pelo sistema de cabeamento). Portanto, pode ser impossível determinar com certeza os valores dos sinais no sistema em um dado momento. Para contornar este problema, será utilizado o filtro de Kalman, para tanto será necessário conhecer dados estatísticos presentes nos sinais de ruído (DUTTON; THOMPSON; BARRACLOUGH, 1997).

Atualmente, o Filtro de Kalman é uma das ferramentas de estimativa mais utilizadas na estimativa de estados de um sistema dinâmico na presença de ruído. É possível estimar os valores reais das medições e os seus valores calculados, atribuindo um peso maior para o valor com o menor grau de incerteza (THRUN; BURGARD; FOX, 2005). A Figura 3-9 mostra a estrutura geral do Filtro de Kalman, bem como as etapas de predição e correção.

O Filtro de Kalman possui muitas aplicações na tecnologia (AUGER et al., 2013) e algumas extensões e generalizações do método são mostradas através da versão estendida (EKF) e Unscented (UKF, do inglês Unscented Kalman Filter) (CRUZ, 2013), (GREWAL; ANDREWS, 2008). 


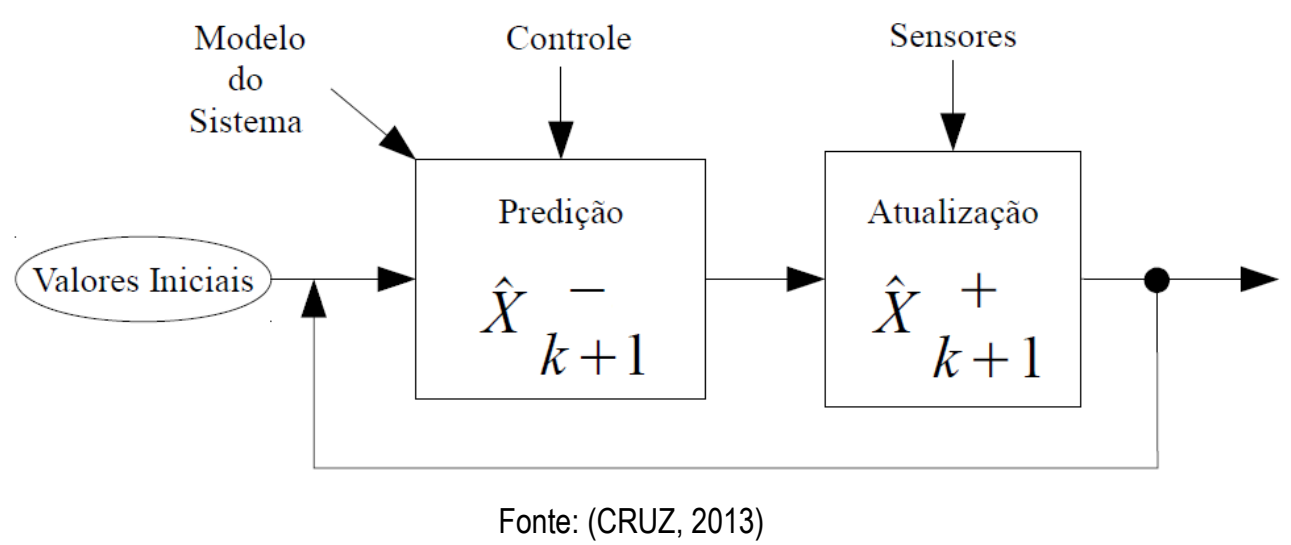

Figura 3-9 - Estrutura geral do filtro de Kalman

O Filtro de Kalman tem excelente desempenho na estimativa de estados em sistemas lineares, porém sua aplicação é complexa em sistemas reais não lineares. $O$ filtro de Kalman Estendido supera essa limitação (CRUZ, 2013).

Considera-se um sistema não linear dado por:

$$
\begin{gathered}
x_{k+1}=f\left(x_{k}, u_{k}, w_{k}\right) \\
y_{k+1}=h\left(x_{k+1}\right)+v_{k+1}
\end{gathered}
$$

onde $f($ ) é uma função não linear do sistema do processo e $h($ ) uma função não linear do sistema de medição. Ambas as funções podem ser usadas para propagar o vetor de estados $x_{k+1}$ e o vetor de saída $y_{k+1}$. As variáveis $u_{k}, w_{k}$ e $v_{k+1}$ estão relacionadas ao controle, ao ruído do processo e ao ruído de medição respectivamente (CRUZ, 2013).

O filtro de Kalman Estendido fornece uma aproximação do valor verdadeiro, representando essa aproximação por uma função gaussiana (THRUN; BURGARD; FOX, 2005), (GREWAL; ANDREWS, 2008).

Se $f()$ é uma função não linear, a projeção da função gaussiana através desta função é tipicamente uma função não gaussiana. Portanto é necessário aproximar a função $f()$ por uma função linear tangente a $f($ ) na média da função gaussiana, de forma que esta distorção não ocorra (CRUZ, 2013).

Uma vez que $f($ ) é linearizada, a mecânica de propagação da esperança é equivalente à do filtro de Kalman. Da mesma forma, é necessário obter uma função 
linear tangente a função de medição de $h()$, mantendo assim a natureza gaussiana da esperança posterior (THRUN; BURGARD; FOX, 2005).

O Filtro de Kalman Estendido utiliza a Expansão de Taylor (primeira ordem) como método de linearização. A expansão de Taylor constrói uma aproximação linear da função $f()$ a partir de seu valor e inclinação conforme mostrado na Equação 3.37:

$$
f^{\prime}\left(x_{k}, u_{k+1}\right)=\frac{\delta f\left(x_{k}, u_{k+1}\right)}{\delta x_{k}}
$$

O Filtro de Kalman Estendido utiliza as linearizações de $f()$ e $h()$ em relação às variáveis de estado e ao ruído do processo, sendo denominadas pelas matrizes Jacobianas das equações $3.38,3.39$ e 3.40 .

$$
\begin{gathered}
F=D f(x)=\left[\begin{array}{cccc}
\frac{\delta f_{1}}{\delta x_{1}} & \frac{\delta f_{1}}{\delta x_{2}} & \cdots & \frac{\delta f_{1}}{\delta x_{n}} \\
\frac{\delta f_{2}}{\delta x_{1}} & \frac{\delta f_{2}}{\delta x_{2}} & \cdots & \frac{\delta f_{2}}{\delta x_{n}} \\
\vdots & \vdots & \ddots & \vdots \\
\frac{\delta f_{n}}{\delta x_{1}} & \frac{\delta f_{n}}{\delta x_{2}} & \cdots & \frac{\delta f_{n}}{\delta x_{n}}
\end{array}\right] \\
H=D h(x)=\left[\begin{array}{cccc}
\frac{\delta h_{1}}{\delta x_{1}} & \frac{\delta h_{1}}{\delta x_{2}} & \cdots & \frac{\delta h_{1}}{\delta x_{n}} \\
\frac{\delta h_{2}}{\delta x_{1}} & \frac{\delta h_{2}}{\delta x_{2}} & \cdots & \frac{\delta h_{2}}{\delta x_{n}} \\
\vdots & \vdots & \ddots & \vdots \\
\frac{\delta h_{n}}{\delta x_{1}} & \frac{\delta h_{n}}{\delta x_{2}} & \cdots & \frac{\delta h_{n}}{\delta x_{n}}
\end{array}\right] \\
W=D f(w)=\left[\begin{array}{cccc}
\frac{\delta f_{1}}{\delta w_{1}} & \frac{\delta f_{1}}{\delta w_{2}} & \cdots & \frac{\delta f_{1}}{\delta w_{n}} \\
\frac{\delta f_{2}}{\delta w_{1}} & \frac{\delta f_{2}}{\delta w_{2}} & \cdots & \frac{\delta f_{2}}{\delta w_{n}} \\
\vdots & \vdots & \ddots & \vdots \\
\frac{\delta f_{n}}{\delta w_{1}} & \frac{\delta f_{n}}{\delta w_{2}} & \cdots & \frac{\delta f_{n}}{\delta w_{n}}
\end{array}\right]
\end{gathered}
$$

As matrizes Jacobianas $F$ e $H$ não são constantes. Porém, caso as mesmas sejam avaliadas em um valor específico do vetor de estados $x=x 0$, as matrizes Jacobianas correspondentes tornam-se constantes. Logo, a etapa da predição é dada pelas Equações 3.41 e 3.42 e a etapa da correção é dada pelas equações 3.43 , 3.44 e 3.45 (CRUZ, 2013). 
Predição:

$$
\begin{gathered}
\hat{X}_{k}^{-}=f\left(\hat{X}_{k-1}^{+}, u_{k-1}\right) \\
P_{k}^{-}=F P_{k-1}^{+} F^{T}+W Q_{k-1} W^{T}
\end{gathered}
$$

Correção:

$$
\begin{gathered}
K_{k}=P_{k}^{-} H^{T}\left[H P_{k}^{-} H^{T}+R_{k}\right]^{-1} \\
\hat{X}_{k}^{+}=\hat{X}_{k}^{-}+K_{k}\left[Y_{k}-h\left(\hat{X}_{k}^{-}\right)\right] \\
P_{k}^{+}=P_{k}^{-}-K_{k} H P_{k}^{-}
\end{gathered}
$$

\subsubsection{Filtro de Kalman Unscented (UKF)}

O filtro de Kalman (KF) é um dos métodos mais utilizados para estimar variáveis de estado devido a sua simplicidade e robustez. Porém, a aplicação do filtro de Kalman a sistemas não lineares pode ser complexa. A abordagem mais comum é utilizar o filtro de Kalman Estendido (EKF), que basicamente lineariza todos os modelos não lineares para que o filtro de Kalman tradicional possa ser aplicado. Conforme mostrado na seção 3.4.1. Embora seja largamente utilizado, existe um consenso de que a implementação do filtro de Kalman Estendido não é simples, pois a linearização pode produzir filtros altamente instáveis, se a hipótese de linearidade local for violada; adicionalmente a derivação de matrizes Jacobianas não são triviais na maioria das aplicações e frequentemente levam a dificuldades significativas de implementação (ST-PIERRE; GINGRAS, 2004).

O Filtro de Kalman Unscented foi proposto com o objetivo de superar os problemas verificados com a utilização do Filtro de Kalman Estendido, possibilitando a obtenção de um estimador com performance equivalente ao filtro de Kalman para sistemas lineares e que representa adequadamente um sistema não linear, porém sem a necessidade dos passos de linearização descritos pelo filtro de Kalman Estendido (JULIER; UHLMANN, 1997). 
Posteriormente, foram propostas algumas modificações para o Filtro de Kalman Unscented com o objetivo de adaptá-lo ao tipo de aplicação (WAN; MERWE; NELSON, 2000).

O Filtro de Kalman Unscented é capaz de realizar estimação de estados em sistemas que contêm não-linearidades, sem a necessidade de se linearizar as funções presentes no modelo (JULIER; UHLMANN, 2004).

A transformação Unscented consiste em se utilizar um conjunto finito de vetores que, ao sofrerem uma transformação não-linear, apresentam média amostral e covariância amostral idênticas às calculadas para o novo conjunto de vetores transformados, supondo uma transformação de $2^{\underline{a}}$ ordem (JULIER; UHLMANN, 1997). A Figura 3-10 mostra como se dá a transformação Unscented.

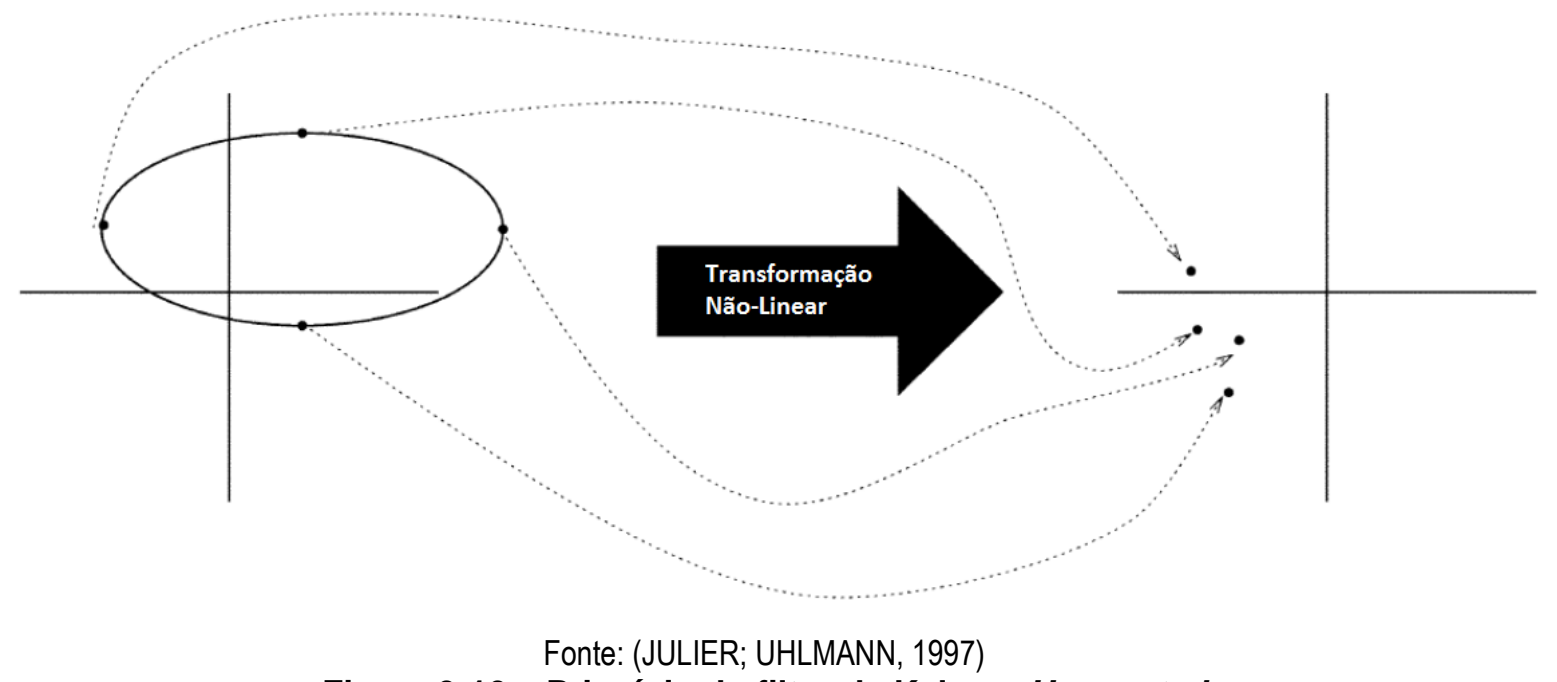

Figura 3-10 - Princípio do filtro de Kalman Unscented

Uma variável aleatória $x$, de dimensão $n$, possui média e covariância dadas por $\bar{x}$ e $P$. Deseja-se calcular a média $\bar{y}$ e a covariância $P^{x y}$ de uma variável aleatória $y$, de dimensão $m$, sendo que $y$ está relacionada a $x$ por meio de uma função não-linear $g$ :

$$
y=g(x)
$$

É mais simples aproximar uma distribuição gaussiana do que linearizar uma função não-linear arbitrária (JULIER; UHLMANN, 1997). Para que uma função de densidade de probabilidade gaussiana possa ser completamente descrita, são necessárias a média e a covariância. Portanto, o objetivo é encontrar alguma parametrização que 
permita a propagação direta da informação por meio das equações não-lineares através da captura da média e da covariância. (JUNIOR, 2003).

É possível obter a parametrização desejada, através da geração de um conjunto de vetores a partir das linhas ou colunas das matrizes $\pm \sqrt{n P}$, ou seja, das matrizes compostas pela raiz quadrada positiva e negativa da matriz de covariância $P$ multiplicada pela dimensão do vetor de variáveis aleatórias $n$. Este conjunto de vetores é dado pela matriz $n \times 2 n$;

$$
\sigma=\left[\begin{array}{ll}
\sqrt{n P} & -\sqrt{n P}
\end{array}\right]
$$

Devido à maneira como é construído, este conjunto de vetores possui covariância amostral igual a $P$ e média amostral zero. Cada vetor é conhecido na literatura como Ponto Sigma (SP) logo, o Filtro de Kalman Unscented é conhecido também como Sigma Points Kalman Filter (SPKF) (MERWE; WAN, 2001).

Para que o novo conjunto de vetores formado possua a mesma média dos vetores $x$ originais, adiciona-se a média $\bar{x}$ à cada coluna da matriz $\sigma$. Como o novo conjunto é simétrico, seus movimentos centrais impares são nulos, fazendo com que os três primeiros momentos sejam iguais aos da distribuição original (JUNIOR, 2003).

É possível ainda adicionar um fator de escala $k$ no cálculo da raiz quadrada da matriz de covariância, sem que o resultado da nova covariância se altere.

O novo conjunto de vetores é gerado da seguinte maneira:

$$
\begin{aligned}
& \sigma=\left[\begin{array}{ll}
\sqrt{(n+k) P} & -\sqrt{(n+k) P}
\end{array}\right] \\
& X^{(0)}=\bar{x} \\
& X^{(i)}=\bar{x}+\sigma^{(i)}
\end{aligned}
$$

Sendo $\sigma^{(i)}$ a iésima coluna da matriz $\sigma$. Desta forma $i$ varia de 1 a $2 n$.

A nova matriz de covariância é dada por:

$$
P=\frac{1}{2(n+k)} \sum_{i=1}^{2 n}\left(X^{(i)}-\bar{x}\right)\left(X^{(i)}-\bar{x}\right)^{T}
$$


Desenvolvendo-se a equação acima, é possível verificar que a covariância de $X$ é igual à covariância original $P$. A transformação Unscented pode ser efetuada seguindo-se o procedimento abaixo (JULIER; UHLMANN, 1996).

Cálculo dos pontos sigmas e novo conjunto de vetores $X$ :

$$
X^{(0)}=\bar{x}
$$

$$
X^{(i)}=\bar{x}+\sigma^{(i)}
$$

Cálculo dos pesos $w$ :

$$
\begin{aligned}
w^{(0)} & =\frac{k}{n+k} \\
w^{(i)} & =\frac{1}{2(n+k)}
\end{aligned}
$$

Cada novo vetor gerado $X^{(i)}$ é propagado usando-se a função não-linear $g$, formando um conjunto de pontos sigmas:

$$
y^{(i)}=g\left(X^{(i)}\right)
$$

A nova média é dada por:

$$
\bar{y}=\sum_{i=0}^{2 n} w^{(i)} y^{(i)}
$$

A covariância do conjunto de vetores transformado $Y^{(i)}$ é:

$$
P^{y y}=\sum_{i=0}^{2 n} w^{(i)}\left(y^{(i)}-\bar{y}\right)\left(Y^{(i)}-\bar{y}\right)^{T}
$$

Observando-se as equações 3.52 e 3.53, verifica-se que o número de pontos sigma $\left(n_{S P}\right)$ é dado por:

$$
n_{S P}=2 n+1
$$

É importante notar que as estimativas das equações 3.57 e 3.58 são exatas somente para transformações até a 2ª ordem (JULIER; UHLMANN, 1996). 


\section{Algoritmo do Filtro de Kalman Unscented}

Passo de predição:

$$
\begin{aligned}
& \hat{X}_{k-1}=\left[\begin{array}{lll}
\hat{x}_{k-1} & \cdots & \hat{x}_{k-1}
\end{array}\right]+\sqrt{c}\left[\begin{array}{ll}
\sqrt{P_{k-1}} & -\sqrt{P_{k-1}}
\end{array}\right] \quad 3.60 \\
& \hat{X}_{k}=f\left(\hat{X}_{k-1}, u_{k-1}\right) \\
& \hat{x}^{-}{ }_{k}=\hat{X}_{k} W_{m} \\
& P^{-}{ }_{k}=\hat{X}_{k} W_{c}\left[\hat{X}_{k}\right]^{T}+Q_{k-1}
\end{aligned}
$$

Passo de atualização:

$$
\begin{aligned}
& \hat{X}_{k}=\left[\begin{array}{lll}
\hat{x}_{k} & \cdots & \hat{x}_{k}
\end{array}\right]+\sqrt{c}\left[\begin{array}{ll}
\sqrt{P_{k}} & -\sqrt{P_{k}}
\end{array}\right] \quad 3.64 \\
& Y_{k}=h\left(\hat{X}_{k}\right) \quad 3.65 \\
& u_{k}=Y^{-}{ }_{k} W_{m} \\
& S_{k}=Y^{-}{ }_{k} W_{m}\left[Y^{-}{ }_{k}\right]^{T}+R_{k} \\
& C_{k}=\widehat{X}_{k}{ }^{-} W_{c}\left[Y^{-}{ }_{k}\right]^{T} \quad 3.68
\end{aligned}
$$

Passo para computar o ganho do filtro $K_{k}$, atualizar a média $\hat{x}_{k}$ e a covariância $P_{k}$ :

$$
\begin{aligned}
& K_{k}=C_{k} S_{k}{ }^{-1} \\
& \hat{x}_{k}=\hat{x}^{-}{ }_{k}+K_{k}\left[y_{k}-u_{k}\right] \\
& P_{k}=P^{-}{ }_{k}+K_{k} S_{k} K_{k}{ }^{T}
\end{aligned}
$$

\subsection{Simulador OLGA ${ }^{\circledR}$}

O simulador computacional OLGA ${ }^{\circledR}$ (Oil-liquid-Gas Analyser) é um simulador numérico unidimensional de escoamento multifásico em dutos e instalações de produções dedicado à indústria de óleo e gás. O software utiliza três equações de continuidade (uma para o gás, uma para o líquido e outra para as gotículas de líquido), duas equações de quantidade de movimento (uma para a fase gás incluindo a fase dispersa e uma para a fase líquida) e 1 equação de energia para a mistura, considerando que todas as fases estão na mesma temperatura (SCHLUMBERGER, 2014).

A solução é dada utilizando-se o método de volumes finitos com integração semiimplícita no tempo, que é o esquema mais adequado para o escoamento multifásico transiente (passos relativamente longos). As propriedades dos fluidos são determinadas utilizando o software PVTsim, que calcula estas propriedades baseado 
nos componentes do óleo e sua quantidade. Este software é capaz de reproduzir o comportamento da golfada em diversos sistemas, incluindo poços sujeitos a golfadas do tipo casing-heading, assim como a maioria das linhas sujeitas a golfadas severas (SCHLUMBERGER, 2014).

O OLGA ${ }^{\circledR}$ é considerado a ferramenta padrão para simulações multifásicas (BURKE; KASHOU, 1995), com aplicações especialmente voltadas ao estudo de golfadas (LAWRENCE et al., 2015), sendo que o mesmo é utilizado com frequência nos estudos comparativos pelo fato de representar bem o sistema real e pelo seu foco em simulações de sistemas com comportamento transiente (MEGLIO, 2011).

A modelagem matemática utilizada no software OLGA ${ }^{\circledR}$ é mostrada de forma detalhada abaixo (BENDIKSEN et al., 1991).

\subsubsection{Modelo Matemático do OLGA ${ }^{\circledR}$}

De forma a simplificar o modelo e a constituição da equação geral da conservação, foram consideradas as seguintes características do escoamento:

- Duto de seção transversal circular de diâmetro constante $D_{i}$ e inclinado de um ângulo $\beta$;

- Unidimensional (1D) com duas fases, gás e líquido, independentes uma da outra;

- Predominância da fase gás na seção do duto;

- Sem transferência de energia entre as fases;

- Regime transiente;

- Padrão de escoamento estratificado;

A Figura 3-11 e a Figura 3-12 abaixo, caracterizam a seção transversal e o perfil de escoamento estratificado do duto. 


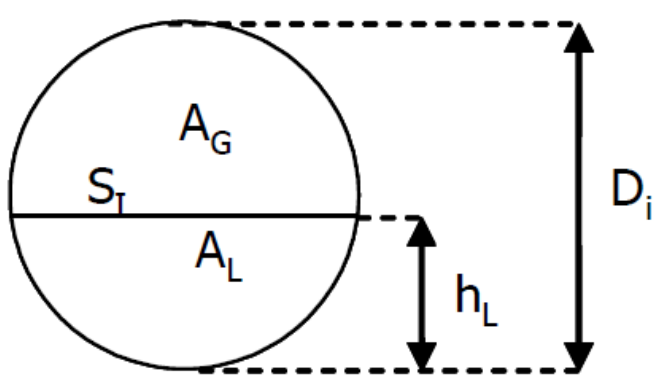

Fonte: (SALES, 2013)

Figura 3-11 - Seção transversal do escoamento.

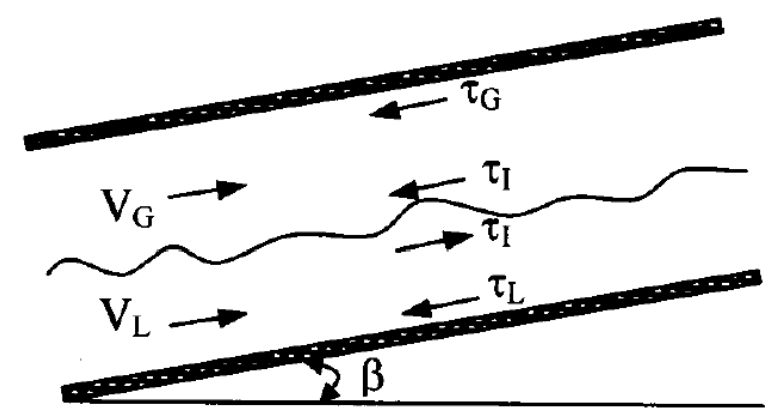

Fonte: (SALES, 2013)

Figura 3-12 - Perfil lateral de escoamento estratificado.

onde $A_{g}$ é a área da seção transversal ocupada pelo gás; $A_{L}$ é a área da seção transversal ocupada pelo líquido; $S_{I}$ é a superfície da interface; $h_{L}$ é a altura ocupada pelo líquido; $D_{i}$ é o diâmetro interno; $V_{g}$ é o volume ocupado pelo gás; $V_{L}$ é o volume ocupado pelo líquido; $\tau_{g}$ é o atrito do gás na parede; $\tau_{L}$ é o atrito do líquido na parede; $\beta$ é a inclinação do tubo em relação a um plano horizontal; e $\tau_{I}$ é o atrito na interface.

\subsubsection{Formulação Matemática}

As equações do modelo de dois fluidos têm sido muito utilizadas na resolução de escoamentos bifásicos em tubulações, particularmente na indústria de petróleo, tendo sido incorporado nos códigos comerciais do OLGA ${ }^{\circledR}$ (BENDIKSEN et al., 1991).

Para os modelos físicos, as equações de continuidade separadas são aplicadas para o gás, para o líquido e para gotículas de líquido, que podem ser acopladas através de transferência de massa interfacial. Esse modelo faz uso das seguintes equações de conservação: 
(i) Uma equação de balanço de massa (continuidade) é utilizada para as três fases;

(ii) Duas equações de quantidade de movimento (uma para a fase gás incluindo a fase dispersa e uma para a fase líquida);

(iii) Uma equação de energia para a mistura. Duas classes de padrões são utilizadas, a saber: distribuído (intermitente ou bolhas) e separado (estratificado ou anular). A transição entre os padrões de escoamento é baseada na fração de área média e determinada de acordo com o conceito de mínimo escorregamento onde é escolhido o padrão de escoamento que leve à menor velocidade do gás.

Conforme mostrado na Figura 3-11 e na Figura 3-12, o duto é de seção circular e os volumes ocupados pelo líquido e pelo gás são dados por $V_{L}$ e $V_{g}$. A razão de volumes pode ser considerada igual a razão de áreas e as frações volumétricas do líquido e do gás, $\alpha_{L}$ e $\alpha_{g}$ podem ser definidas como (SALES, 2013):

$$
\begin{aligned}
& \alpha_{L}=\frac{A_{L}}{A} \\
& \alpha_{g}=\frac{A_{g}}{A}
\end{aligned}
$$

Onde as frações volumétricas, $\alpha_{L}$ e $\alpha_{g}$, estão sujeitas a seguinte condição:

$$
\alpha_{L}+\alpha_{g}=1
$$

\subsubsection{Equações de Balanço de Massa}

Para a fase gás:

$$
\frac{\partial}{\partial t}\left(\alpha_{g} \rho_{g}\right)=-\frac{1}{A} \frac{\partial}{\partial z}\left(A \alpha_{g} \rho_{g} v_{g}\right)+\psi_{g}+G_{g}
$$

Para a fase líquida:

$$
\frac{\partial}{\partial t}\left(\alpha_{L} \rho_{L}\right)=-\frac{1}{A} \frac{\partial}{\partial z}\left(A \alpha_{L} \rho_{L} v_{L}\right)+\psi_{g} \frac{\alpha_{L}}{\alpha_{L}+\alpha_{D}}-\psi_{e}+\psi_{d}+G_{L}
$$

Para a fase de gotículas de líquido:

$$
\frac{\partial}{\partial t}\left(\alpha_{D} \rho_{L}\right)=-\frac{1}{A} \frac{\partial}{\partial z}\left(A \alpha_{D} \rho_{L} v_{D}\right)-\psi_{g} \frac{\alpha_{D}}{\alpha_{L}+\alpha_{D}}+\psi_{e}-\psi_{d}+G_{D}
$$

Para os termos das equações 3.75, 3.76 e 3.77 tem-se $\alpha_{g}$ - Fração volumétrica do gás; $\alpha_{L}$ - Fração volumétrica do líquido; $\alpha_{D}$ - Fração volumétrica das gotas de líquido; $\rho$ - Massa específica; $v$ - Velocidade; $p$ - Pressão; $A$ - Área da seção transversal do 
tubo; $\psi_{g}$ - Taxa de transferência de massa entre as fases; $\psi_{e}$ - Taxa de arrastamento; $\psi_{d}$ - Taxa de deposição; $G_{g}$ - Fonte de massa da fase gás; $G_{L}$ - Fonte de massa da fase líquida; e $G_{D}$ - Fonte de massa da fase dispersa de gotículas de líquido. Os subscritos $g, L, i$ e $D$ indicam gás, líquido, interface e deposição de gotas.

\subsubsection{Equações de Quantidade de Movimento}

Para a fase gás:

$$
\begin{aligned}
\frac{\partial}{\partial t}\left(\alpha_{g} \rho_{g} v_{g}\right)= & -\alpha_{g}\left(\frac{\partial p}{\partial z}\right)-\frac{1}{A} \frac{\partial}{\partial z}\left(A \alpha_{g} \rho_{g} v_{g}{ }^{2}\right)-\lambda_{g} \frac{1}{2} \rho_{g}\left|v_{g}\right| v_{g} \frac{S_{g}}{4 A}-\lambda_{i} \frac{1}{2} \rho_{g}\left|v_{r}\right| v_{r} \frac{S_{I}}{4 A} \\
& +\alpha_{g} \rho_{g} g \cos \beta+\psi_{g} v_{a}-F_{D}
\end{aligned}
$$

Para a fase gotículas de líquido:

$$
\begin{gathered}
\frac{\partial}{\partial t}\left(\alpha_{D} \rho_{L} v_{D}\right)=-\alpha_{D}\left(\frac{\partial p}{\partial z}\right)-\frac{1}{A} \frac{\partial}{\partial z}\left(A \alpha_{D} \rho_{L} v_{D}^{2}\right)+\alpha_{D} \rho_{L} g \cos \beta-\psi_{g} \frac{\alpha_{D}}{\alpha_{L}+\alpha_{D}} v_{a}+\psi_{e} v_{i}- \\
\psi_{d} v_{D}+F_{D}
\end{gathered}
$$

As equações 3.78 e 3.79 foram combinadas para produzir um salto tal que o termo de arrasto representado por $F_{D}$ na relação entre gás/gotículas se anula. Assim tem-se somente uma equação de balanço de quantidade de movimento para a fase gás incluindo a fase dispersa de gotículas de líquido, representada pela equação 3.80 abaixo: 
Para a combinação gás/gotículas de líquido:

$$
\begin{aligned}
\frac{\partial}{\partial t}\left(\alpha_{g} \rho_{g} v_{g}+\right. & \left.\alpha_{D} \rho_{L} v_{D}\right) \\
& =-\left(\alpha_{g}+\alpha_{D}\right)\left(\frac{\partial p}{\partial z}\right)-\frac{1}{A} \frac{\partial}{\partial z}\left(A \alpha_{g} \rho_{g} v_{g}{ }^{2}+A \alpha_{D} \rho_{L} v_{D}^{2}\right)-\lambda_{g} \frac{1}{2} \rho_{g}\left|v_{g}\right| v_{g} \frac{S_{g}}{4 A} \\
& -\lambda_{i} \frac{1}{2} \rho_{g}\left|v_{r}\right| v_{r} \frac{S_{i}}{4 A}+\left(\alpha_{g} \rho_{g}+\alpha_{D} \rho_{L}\right) g \cos \beta+\psi_{g} \frac{\alpha_{L}}{\alpha_{L}+\alpha_{D}} v_{a}+\psi_{e} v_{i} \\
& -\psi_{d} v_{D}
\end{aligned}
$$

Para a fase líquida:

$$
\begin{aligned}
\frac{\partial}{\partial t}\left(\alpha_{L} \rho_{L} v_{L}\right)= & -\alpha_{L}\left(\frac{\partial p}{\partial z}\right)-\frac{1}{A} \frac{\partial}{\partial z}\left(A \alpha_{L} \rho_{L} v_{L}^{2}\right)-\lambda_{L} \frac{1}{2} \rho_{L}\left|v_{L}\right| v_{L} \frac{S_{L}}{4 A}-\lambda_{i} \frac{1}{2} \rho_{g}\left|v_{r}\right| v_{r} \frac{S_{i}}{4 A} \\
& +\alpha_{L} \rho_{L} g \cos \beta+\psi_{g} \frac{\alpha_{L}}{\alpha_{L}+\alpha_{D}} v_{a}-\psi_{e} v_{i}+\psi_{d} v_{D} \\
& -\alpha_{L} d\left(\rho_{L}-\rho_{g}\right) g \frac{\partial \alpha_{L}}{\partial z} \sin \beta
\end{aligned}
$$

Nas equações 3.78 a 3.81, $\beta$ corresponde a inclinação do tubo com a vertical e $S_{g}$, $S_{L}$ e $S_{i}$ são respectivamente, o perímetro molhado do gás, do líquido e da interface. Supõe-se que a fonte interna $G_{f}$ entra com um ângulo de $90^{\circ}$ na parede do tubo, caminhando sem impulso líquido. Com isso pode-se considerar:

- Na evaporação do filme líquido:

$$
v_{a}=v_{L} \text { para } \psi_{g}>0
$$

- Na evaporação das gotas de líquido:

$$
v_{a}=v_{D} \text { para } \psi_{g}>0
$$

- Na condensação:

$$
v_{a}=v_{g} \text { para } \psi_{g}<0
$$

As equações de conservação acima podem ser aplicadas para todos os tipos de regimes de escoamento.

A velocidade relativa $v_{r}$ é definida pela seguinte equação de deslizamento

$$
v_{g}=R_{D}\left(v_{L}+v_{r}\right)
$$


Onde $R_{D}$ é a uma taxa de distribuição de deslizamento causado por uma distribuição desigual de fases e velocidades em toda a seção transversal da tubulação, como esboçada na seção seguinte.

A velocidade das gotículas é similarmente definida por:

$$
v_{D}=v_{g}-v_{O D} \cos \alpha
$$

onde $v_{O D}$ é a velocidade de queda da gotícula. A velocidade de interface $v_{i}$ é aproximada para $v_{L}$, que é a velocidade do líquido.

\subsubsection{Equação de Energia da Mistura}

Para a energia da mistura tem-se:

$$
\begin{aligned}
\frac{\partial}{\partial t}\left[m _ { g } \left(E_{g}+\frac{1}{2}\right.\right. & \left.\left.v_{g}^{2}+g h\right)+m_{L}\left(E_{L}+\frac{1}{2} v_{L}^{2}+g h\right)+m_{D}\left(E_{D}+\frac{1}{2} v_{D}^{2}+g h\right)\right] \\
= & -\frac{\partial}{\partial z}\left[m_{g} v_{g}\left(H_{g}+\frac{1}{2} v_{g}^{2}+g h\right)+m_{L} v_{L}\left(H_{L}+\frac{1}{2} v_{L}^{2}+g h\right)\right. \\
& \left.+m_{D} v_{D}\left(H_{D}+\frac{1}{2} v_{D}^{2}+g h\right)\right]+H_{S}+U
\end{aligned}
$$

onde $E$ é a energia interna por unidade de massa, $h$ é a elevação, $H_{S}$ é a entalpia da fonte de massa e $U$ é a transferência de calor da parede do tubo.

\subsubsection{Propriedades dos Fluidos e Transferência de Fase}

Todas as propriedades (massas específicas, compressibilidades, viscosidades, tensão superficial, entalpias, capacidades de calor e condutividade térmica) são dadas em tabelas em função da pressão e temperatura e os valores reais em um determinado ponto no tempo e espaço são encontrados pela interpolação nestas tabelas (BENDIKSEN et al., 1991). Essas tabelas são geradas previamente à simulação do OLGA ${ }^{\circledR}$, utilizando qualquer pacote de propriedades dos fluidos, baseadas nas equações de Peng-Robinson, Soave-Redlich-Kwong ou outra equação de estado, em conformidade com o formato da tabela especificada (SALES, 2013).

Considera-se que, a composição total da mistura é constante no tempo ao longo da tubulação, enquanto que as composições do gás e do líquido mudam com a pressão e a temperatura, como resultado da transferência de massa interfacial. Em sistemas 
reais, a diferença de velocidade entre as fases de gás e óleo podem causar mudanças na composição total da mistura. Isso pode ser totalmente contabilizado somente para um modelo de composição (BENDIKSEN et al., 1991).

\subsubsection{Transferência de Massa Interfacial}

A aplicação do modelo de transferência de massa na interface pode tratar tanto condensação normal quanto evaporação e condensação retrógrada, em que uma fase pesada condensa a partir da fase de gás com a queda de pressão (BENDIKSEN et al., 1991). A fração mássica de gás em condições de equilíbrio pode ser definida como:

$$
R_{S}=\frac{m_{g}}{\left(m_{g}+m_{L}+m_{D}\right)}
$$

A taxa de transferência de massa poderá ser expressa da seguinte maneira:

$$
\psi_{g}=\left[\left(\frac{\partial R_{S}}{\partial p}\right)_{T} \frac{\partial p}{\partial t}+\left(\frac{\partial R_{S}}{\partial p}\right)_{T} \frac{\partial p}{\partial z} \frac{\partial z}{\partial t}+\left(\frac{\partial R_{S}}{\partial p}\right)_{P} \frac{\partial T}{\partial z}+\left(\frac{\partial R_{S}}{\partial p}\right)_{P} \frac{\partial T}{\partial t} \frac{\partial z}{\partial t}\right]\left(m_{g}+m_{L}+m_{D}\right)
$$

O termo $\left(\frac{\partial R_{S}}{\partial p}\right)_{T} \frac{\partial p}{\partial t}$ representa a transferência de fase a partir de uma massa presente em uma seção devido a mudança de pressão na referida secção. Já o termo $\left(\frac{\partial R_{S}}{\partial p}\right)_{T} \frac{\partial p}{\partial z} \frac{\partial z}{\partial t}$ representa a transferência de massa causada pela massa que flui de uma seção para a outra. 


\subsection{Modelos Dinâmicos Simplificados}

Ao estudar um escoamento em golfadas, o foco deve ser direcionado a capturar a dinâmica da formação da golfada, não a golfada em si, ou seja, o objetivo é evitar o regime de escoamento em golfadas ao invés de determinar o comprimento ou o formato das mesmas. Ao invés de lidar com modelos complexos, como os desenvolvidos no simulador $\mathrm{OLGA}^{\circledR}$, é necessário um modelo dinâmico simplificado para a análise de controlabilidade e projeto do controlador. Alguns dos principais modelos encontrados na literatura estão citados a seguir: "Modelo de Jahanshahi" (JAHANSHAHI; SKOGESTAD, 2011), "Modelo de Storkaas" (STORKAAS; SKOGESTAD, 2003), "Modelo de Eikrem", "Modelo de Kaasa" (KAASA et al., 2007), "Modelo de Nydal" (SILVA; DESSEN; NYDAL, 2010) e "Modelo de Di Meglio" (MEGLIO; KAASA; PETIT, 2009). Os modelos citados foram simulados no domínio do tempo e seus resultados comparados ao modelo OLGA ${ }^{\circledR}$ com o objetivo de avaliar os cinco aspectos abaixo, listados em ordem de importância (JAHANSHAHI; SKOGESTAD, 2011):

- Abertura crítica da válvula choke para a formação de golfadas

- Frequência das oscilações com a válvula choke na abertura crítica

- Resposta dinâmica para uma mudança em degrau da abertura da válvula choke

- Pressão e vazão do sistema para o regime permanente

- Pressões e vazões mínima e máxima das oscilações durante o regime de golfadas

Os modelos simplificados também foram analisados no domínio da frequência, onde foram consideradas a localização dos polos instáveis e zeros instáveis (RHP) do modelo. Ao final do comparativo, os modelos que apresentaram os melhores resultados e as melhores predições das pressões e vazões mínimas e máximas para o regime permanente quando comparadas aos resultados do OLGA ${ }^{\circledR}$, foram o "Modelo de Jahanshahi" e o "Modelo de Di Meglio".

\footnotetext{
${ }^{1}$ EIKREM, G. O. 2008. "Eikrem Riser Model"

http://www.nt.ntnu.no/users/skoge/diplom/prosjekt08/tuvnes
} 
Porém o "Modelo de Jahanshahi" apresentou um melhor ajuste para uma resposta em degrau da pressão de topside (JAHANSHAHI; SKOGESTAD, 2011). Portanto, optouse neste trabalho pela utilização do "Modelo de Jahanshahi", pois considera-se que este é o modelo simplificado mais representativo do sistema real e apresenta vantagens sobre os demais modelos encontrados na literatura. Este modelo será descrito a seguir, em detalhes.

\section{7. "Modelo de Jahanshahi" para um Sistema "Pipeline-Riser"}

\subsubsection{Equações de Balanço de Massa para um Sistema “Pipeline-Riser”}

As equações diferenciais 3.90, 3.91, 3.92 e 3.93 mostradas abaixo representam as equações de conservação de massa das fases gasosa e líquida do sistema "pipelineriser" mostrado na Figura 3-13 e na Figura 3-14.

$$
\begin{aligned}
& \frac{d m_{g, p}}{d t}=w_{g, \text { in }}-w_{g, r b} \\
& \frac{d m_{l, p}}{d t}=w_{l, \text { in }}-w_{l, r b} \\
& \frac{d m_{g, r}}{d t}=w_{g, r b}-w_{g, \text { out }} \\
& \frac{d m_{l, r}}{d t}=w_{l, r b}-w_{l, \text { out }}
\end{aligned}
$$

As quatro variáveis de estado do modelo são: $m_{g, p}$ massa de gás no pipeline [kg] $m_{l, p}$ massa de líquido no pipeline $[\mathrm{kg}], m_{g, r}$ massa de gás no riser $[\mathrm{kg}]$ e $m_{l, r}$ massa de líquido no riser $[\mathrm{kg}]$. 


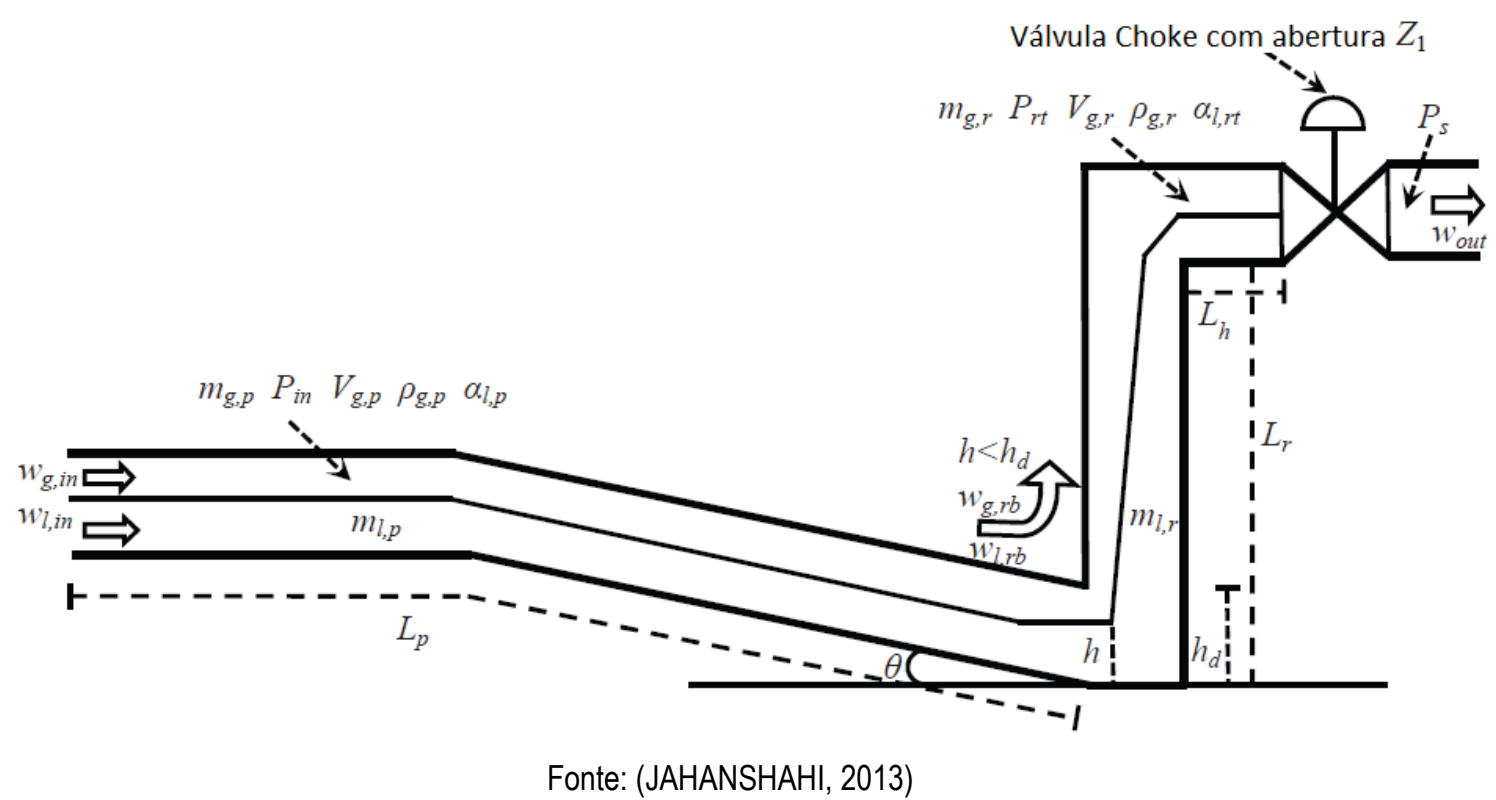

Figura 3-13 - Representação simplificada do escoamento (Sem bloqueio de líquido)

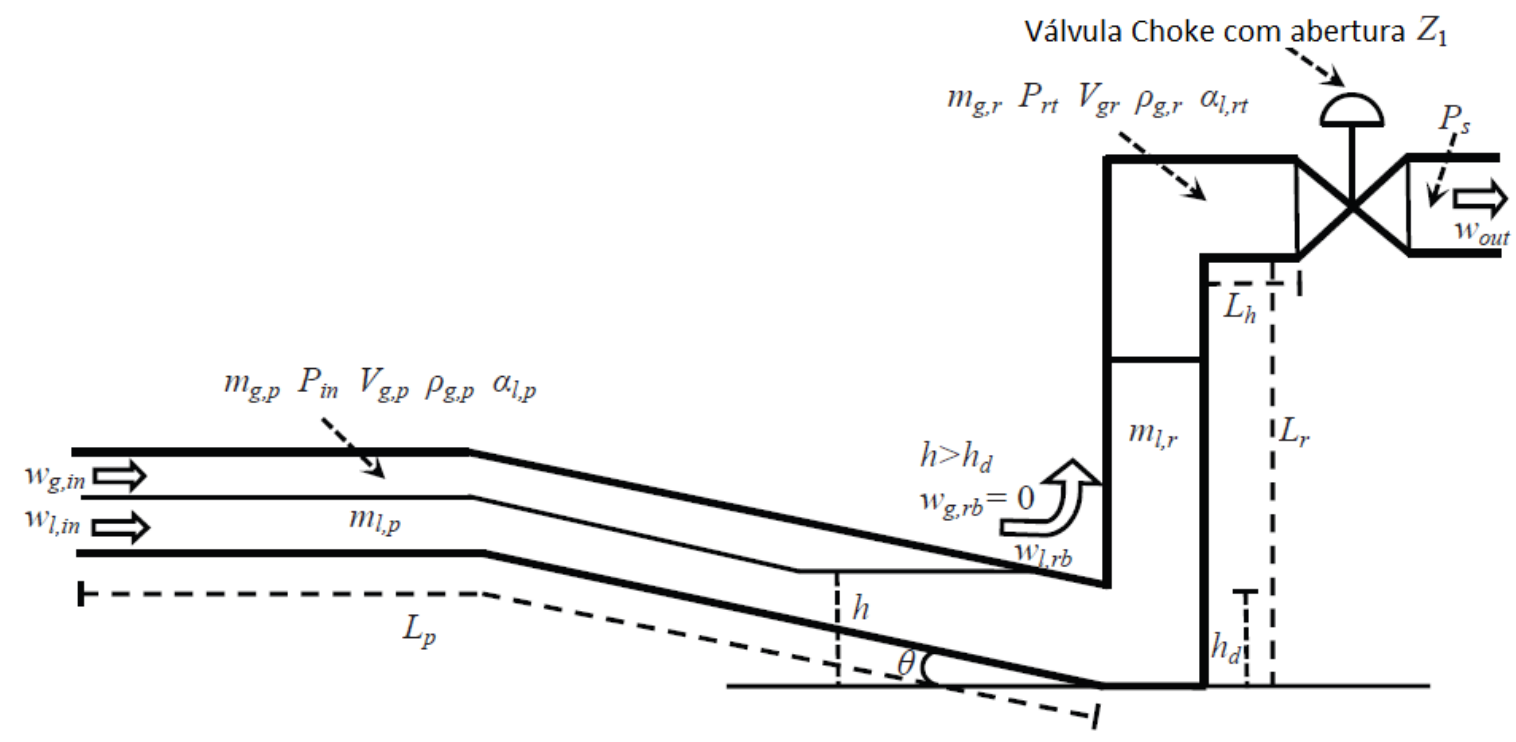

Fonte: (JAHANSHAHI, 2013)

Figura 3-14 - Representação simplificada do bloqueio de líquido na base do riser, levando a golfada severa.

Os principais parâmetros utilizados no modelo estão descritos na Tabela 2. Quatro dos parâmetros encontrados nesta tabela são utilizados para sintonia do modelo, conforme abaixo: 
- $K_{h}$ : Fator de correção do nível de líquido no pipeline

- $C_{v 1}$ : Constante da válvula choke de produção

- $K_{g}$ : Coeficiente para a vazão de gás na base do riser

- $K_{l}$ : Coeficiente para a vazão de líquido na base do riser

\subsubsection{Condições de Contorno na Entrada}

Nas equações 3.90 e $3.91, w_{g, \text { in }}$ e $w_{l, i n}$ representam as vazões mássicas de entrada do gás e do líquido, respectivamente. A princípio, é assumido que estas vazões são constantes, mas esta condição de contorno pode ser posteriormente alterada por exemplo, para uma vazão de entrada variável com a pressão.

\subsubsection{Condições de Contorno na Saída}

Considera-se como condição de contorno na saída, que a pressão na saída é constante (pressão de separação, $P_{S}$ ). Logo, é possível representar a vazão de saída para uma mistura bifásica pela equação da válvula choke (JAHANSHAHI, 2013):

$$
w_{\text {out }}=C_{v 1} \cdot f\left(z_{1}\right) \sqrt{\rho_{r t} \cdot \max \left(P_{r t}-P_{s}, 0\right)}
$$

Sendo $z 1$, a abertura normalizada da válvula choke $(0<z 1<1)$ e $f\left(z_{1}\right)$, a equação característica da válvula.

Nas simulações, é considerada uma válvula linear, ou seja, $f\left(z_{1}\right)=z_{1}$. Esta premissa pode ser alterada para outros tipos de válvulas.

As vazões mássicas de saída são calculadas (JAHANSHAHI, 2013) conforme abaixo:

$$
\begin{aligned}
& w_{l, \text { out }}=\alpha_{l, r t}^{m} \cdot w_{\text {out }} \\
& w_{g, \text { out }}=\left(1-\alpha_{l, r t}^{m}\right) \cdot w_{\text {out }}
\end{aligned}
$$

A fração mássica $\alpha_{l, r t}^{m}$ de líquido no topo do riser é dada por: 


$$
\alpha_{l, r t}^{m}=\frac{\alpha_{l, r t} \cdot \rho_{l}}{\alpha_{l, r t} \cdot \rho_{l}+\left(1-\alpha_{l, r t}\right) \cdot \rho_{g, r}}
$$

A densidade da mistura bifásica no topo do riser é dada por:

$$
\rho_{r t}=\alpha_{l, r t} \cdot \rho_{l}+\left(1-\alpha_{l, r t}\right) \cdot \rho_{g, r}
$$

A fração volumétrica de líquido, $\alpha_{l, r t}$ é calculada pela equação:

$$
\alpha_{l, r t}=2 . \bar{\alpha}_{l, r}-\alpha_{l, r b}=\frac{2 \cdot m_{l, r}}{V_{r} \cdot \rho_{l}}-\frac{A_{l}}{A_{p}}
$$

\subsubsection{Modelo do Pipeline}

A fração volumétrica de líquido na seção do pipeline $\alpha_{l}$ é dada pela fração mássica de líquido $\alpha_{l}^{m}$ e pela densidade das fases líquida e gasosa:

$$
\alpha_{l}=\frac{\alpha_{l}^{m} / \rho_{l}}{\alpha_{l}^{m} / \rho_{l}+\left(1-\alpha_{l}^{m}\right) / \rho_{g}}
$$

A fração mássica média de líquido na seção do pipeline é dada pelas vazões mássicas na entrada:

$$
\bar{\alpha}_{l, p}^{m}=\frac{w_{l, i n}}{w_{g, i n}+w_{l, i n}}
$$

Então, a fração volumétrica média de líquido no pipeline é:

$$
\bar{\alpha}_{l, p}=\frac{\bar{\rho}_{g, p} \cdot w_{l, i n}}{\bar{\rho}_{g, p} \cdot w_{l, i n}+\rho_{l} \cdot w_{g, i n}}
$$

A densidade do gás $\bar{\rho}_{g, p}$ é calculada com base na pressão nominal (em regime permanente) no pipeline. Para efeitos de simplicidade do modelo, assumiu-se que o gás é ideal:

$$
\bar{\rho}_{g, p}=\frac{P_{i n, n o m} \cdot M_{g}}{R \cdot T_{p}}
$$

Pressão de entrada nominal $P_{\text {in,nom }}$ é calculada a partir de uma inicialização em regime permanente do modelo. Portanto, para vazões de entrada constantes, obtémse $\bar{\alpha}_{l, p}$ também constante. A área da seção transversal do pipeline é dada por:

$$
A_{p}=\frac{\pi}{4} \cdot D_{p}^{2}
$$


onde $D_{p}$ é o diâmetro do pipeline, então o volume do pipeline é dado por $V_{p}=A_{p}$. $L_{p}$. Considerando uma distribuição homogênea do gás e do líquido ao longo do pipeline, a massa de líquido é dada por:

$$
\bar{m}_{l, p}=\rho_{l} \cdot V_{p} \cdot \bar{\alpha}_{l, p}
$$

Portanto, o nível de líquido no ponto mais baixo do pipeline é dado aproximadamente por $\bar{h} \approx h_{d} \cdot \bar{\alpha}_{l, p}$, onde $h_{d}=D_{p} \cdot \cos \theta$ é a abertura ao fluxo na base do riser e $\theta$ a inclinação do pipeline, ou seja:

$$
\bar{h}=K_{h} \cdot h_{d} \cdot \bar{\alpha}_{l, p}
$$

onde $K_{h}$ é o fator de correção em torno da unidade que pode ser utilizado para o ajuste fino do modelo. Se o conteúdo de líquido no pipeline aumentar de $\Delta m_{l, p}$, o mesmo começa a preencher o pipeline a partir do ponto mais baixo e um comprimento do pipeline igual a $\Delta L$ será ocupado unicamente por líquido.

A variação de massa do líquido no pipeline é calculada por:

$$
\Delta m_{l, p}=m_{l, p}-\bar{m}_{l, p}=\Delta L \cdot A_{p} \cdot\left(1-\bar{\alpha}_{l, p}\right) \cdot \rho_{l}
$$

O nível de líquido no pipeline é $h=\overline{\mathrm{h}}+\Delta L$. $\operatorname{sen} \theta$, ou seja:

$$
h=\bar{h}+\left(\frac{m_{l, p}-\bar{m}_{l, p}}{A_{p} \cdot\left(1-\bar{\alpha}_{l, p}\right) \cdot \rho_{l}}\right) \cdot \operatorname{sen} \theta
$$

Assim, o nível de líquido no pipeline $h$ pode ser escrito como uma função da massa de líquido no pipeline $m_{l, p}$, que é uma variável de estado do modelo. Os demais parâmetros da equação 3.108 são constantes.

A densidade do gás no pipeline é dada por:

$$
\rho_{g, p}=\frac{m_{g, p}}{V_{g, p}}
$$

onde o volume ocupado pelo gás no pipeline é:

$$
V_{g, p}=V_{p}-m_{l, p} / \rho_{l}
$$

A pressão na entrada do pipeline, assumindo que o gás é ideal, é dado por:

$$
P_{\text {in }}=\frac{\rho_{g, p} \cdot R \cdot T_{p}}{M_{g}}
$$


Para o cálculo das perdas por fricção no pipeline, apenas o líquido é considerado:

$$
\Delta P_{f p}=\frac{\bar{\alpha}_{l, p} \cdot \lambda_{p} \cdot \rho_{l} \cdot \bar{U}_{s l, i n}^{2} \cdot L_{p}}{2 \cdot D_{p}}
$$

O fator de friç̧ão $\lambda_{p}$ do pipeline pode ser considerado como uma aproximação explícita da equação implícita de Colebrook-White. Esta equação foi adotada por ser considerada a mais precisa lei de resistência ao escoamento, sendo utilizada como padrão referencial:

$$
\frac{1}{\sqrt{\lambda_{p}}}=-1,8 \cdot \log _{10} \cdot\left[\left(\frac{\epsilon / D_{p}}{3,7}\right)^{1,11}+\frac{6,9}{R e_{p}}\right]
$$

onde o número de Reynolds é:

$$
R e_{p}=\frac{\rho_{l} \cdot \bar{U}_{s l, i n} \cdot D_{p}}{\mu}
$$

O termo $\mu$ é a viscosidade do líquido. A velocidade superficial $\bar{U}_{s l, i n}$, do líquido é:

$$
\bar{U}_{s l, i n}=\frac{4 \cdot w_{l, i n}}{\pi \cdot D_{p}^{2} \cdot \rho_{l}}
$$

3.7.5. Modelo do Riser

O volume total do riser é dado por:

$$
V_{r}=A_{r} .\left(L_{r}+L_{h}\right)
$$

onde a sua área da seção transversal é

$$
A_{r}=\frac{\pi}{4} D_{r}^{2}
$$

E o volume ocupado pelo gás no riser é dado por:

$$
V_{g, r}=V_{r}-m_{l, r} / \rho_{l}
$$

A densidade do gás no riser é dada por:

$$
\rho_{g, r}=\frac{m_{g, r}}{V_{g, r}}
$$

E a pressão no topo do riser, dada pela lei dos gases ideais é:

$$
P_{r t}=\frac{\rho_{g, r} \cdot R \cdot T_{r}}{M_{g}}
$$

A fração volumétrica média de líquido no riser é: 


$$
\bar{\alpha}_{l, r}=\frac{m_{l, r}}{V_{r} \cdot \rho_{l}}
$$

A densidade média da mistura no riser é:

$$
\bar{\rho}_{m, r}=\frac{m_{g, r}+m_{l, r}}{V_{r}}
$$

A perda por fricção no riser é:

$$
\Delta P_{f r}=\frac{\bar{\alpha}_{l, r} \cdot \lambda_{r} \cdot \bar{\rho}_{m, r} \cdot \bar{U}_{m \cdot\left(L_{r}+L_{h}\right)}^{2}}{2 \cdot D_{r}}
$$

O fator de fricção do riser, utilizando-se da mesma correlação adotada para o pipeline é:

$$
\frac{1}{\sqrt{\lambda_{r}}}=-1,8 \cdot \log _{10} \cdot\left[\left(\frac{\epsilon / D_{r}}{3,7}\right)^{1,11}+\frac{6,9}{R e_{r}}\right]
$$

O número de Reynolds para o escoamento no riser é dado por:

$$
R e_{r}=\frac{\bar{\rho}_{m, r} \cdot \bar{U}_{m} \cdot D_{r}}{\mu}
$$

Sendo as velocidades da mistura no riser dadas por:

$$
\begin{aligned}
& \bar{U}_{m}=\bar{U}_{s l, r}+\bar{U}_{s g, r} \\
& \bar{U}_{s l, r}=\frac{w_{l, i n}}{\rho_{l} \cdot A_{r}} \\
& \bar{U}_{s g, r}=\frac{w_{g, i n}}{\rho_{g, r} \cdot A_{r}}
\end{aligned}
$$

3.7.6. Modelo de Escoamento de Gás na Base do Riser

Como ilustrado na Figura 3-14, quando o nível de líquido na seção do pipeline ultrapassa a abertura do duto na base do riser $\left(h>h_{d}\right)$, o líquido causa um bloqueio do gás, ou seja, a vazão de gás $w_{g, r b}$ na base do riser é zero.

$$
w_{g, r b}=0, \quad h \geq h_{d}
$$

Quando o líquido não está bloqueando a base do riser $\left(h<h_{d}\right)$ conforme Figura 3-13, o gás irá fluir do volume $V_{g, p}$ para $V_{g, r}$ com a vazão mássica $w_{g, r b}[\mathrm{~kg} / \mathrm{s}]$ que é dada por uma "equação de orifício" (SKOGESTAD, 2009): 


$$
w_{g, r b}=K_{g} \cdot A_{g} \cdot \sqrt{\rho_{g, p} \cdot \Delta P_{g}}, \quad h<h_{d}
$$

onde:

$$
\Delta P_{g}=P_{i n}-\Delta P_{f p}-P_{r t}-\bar{\rho}_{m, r} \cdot g \cdot L_{r}-\Delta P_{f r}
$$

A área livre $A_{g}$ para o escoamento de gás pode ser calculada utilizando funções trigonométricas, mas por simplicidade, é utilizada neste modelo, uma aproximação quadrática.

$$
\begin{aligned}
& A_{g}=A_{p} \cdot\left(\frac{h_{d}-h}{h_{d}}\right)^{2}, h<h_{d} \\
& A_{g}=0, h \geq h_{d}
\end{aligned}
$$

\subsubsection{Modelo de Escoamento de Líquido na Base do Riser}

A vazão mássica de líquido na base do riser é também descrita por uma "equação de orifício":

$$
w_{l, r b}=K_{l} \cdot A_{l} \cdot \sqrt{\rho_{l} \cdot \Delta P_{l}}
$$

onde:

$$
\Delta P_{l}=P_{i n}-\Delta P_{f p}+\rho_{l} \cdot g \cdot h-P_{r t}-\bar{\rho}_{m, r} \cdot g \cdot L_{r}-\Delta P_{f r}
$$

e também:

$$
A_{l}=A_{p}-A_{g}
$$

\subsubsection{Modelo de para Distribuição das Fases na Saída da Válvula Choke}

Para o cálculo das vazões mássicas de cada fase, deve-se conhecer a distribuição das fases no topo do riser. A fração volumétrica de líquido no topo do riser $\left(\alpha_{l t}\right)$, é calculada considerando-se que, em um duto com escoamento bifásico vertical, há uma relação aproximadamente linear entre a pressão e a fração volumétrica de líquido. Adicionalmente, o gradiente de pressão é assumido como constante ao longo do riser para regimes de escoamento estáveis (sem golfadas), ou seja, o gradiente da fração de líquido é constante $\left(\frac{\partial \alpha_{l r}}{\partial y}=\right.$ constante $)$. Logo, a fração volumétrica média de líquido no riser é dada por: 


$$
\bar{\alpha}_{l r}=\frac{\alpha_{l, r b}+\alpha_{l, r t}}{2}
$$

Esta fração volumétrica $\bar{\alpha}_{l, r}$ do líquido no riser também é dada pela equação 3.121. A fração $\alpha_{l, r b}$ na base do riser é determinada pela área ocupada pelo líquido nesta base do riser:

$$
\alpha_{l, r b}=\frac{A_{l}}{A_{p}}
$$

Então, a fração volumétrica de líquido no topo do riser é:

$$
\alpha_{l, r t}=2 . \bar{\alpha}_{l, r}-\alpha_{l, r b}=\frac{2 . m_{l, r}}{V_{r} \cdot \rho_{l}}-\frac{A_{l}}{A_{p}}
$$

Tabela 2 - Parâmetros do sistema pipeline-riser

\begin{tabular}{cccc}
\hline Símbolo & Descrição & Valor & Unidades \\
\hline$R$ & Constante universal dos gases & 8314 & $\mathrm{~J} /(\mathrm{kmol} . \mathrm{K})$ \\
$g$ & Gravidade & 9,81 & $\mathrm{~m} / \mathrm{s}^{2}$ \\
$\mu$ & Viscosidade do líquido & $1426 \times 10^{-4}$ & $\mathrm{Pa.s}$ \\
$\rho_{l}$ & Densidade do líquido & 832,2 & $\mathrm{~kg} / \mathrm{m}^{3}$ \\
$M_{g}$ & Peso molecular do gás & 20 & $\mathrm{~g}$ \\
$T_{p}$ & Temperatura do pipeline & 293,15 & $\mathrm{~K}$ \\
$D_{p}$ & Diâmetro do pipeline & 0,12 & $\mathrm{~m}$ \\
$L_{p}$ & Comprimento do pipeline & 4300 & $\mathrm{~m}$ \\
$D_{r}$ & Diâmetro do riser & 0,1 & $\mathrm{~m}$ \\
$L_{r}$ & Comprimento do riser & 300 & $\mathrm{~m}$ \\
$L_{r}$ & Comprimento da seção horizontal no topo do riser & 100 & $\mathrm{~m}$ \\
$P_{S}$ & Pressão do separador & 50,1 & $b a r$ \\
$K_{h}$ & Fator de correção de nível & 0,7 & - \\
$K_{g}$ & Coeficiente para a vazão de gás na base do riser & $3,49 \times 10^{-2}$ & - \\
$K_{l}$ & Coeficiente para a vazão de líquido na base do riser & $2,81 \times 10^{-1}$ & - \\
$C_{v 1}$ & Constante da válvula choke de produção & $1,16 \times 10^{-2}$ & -
\end{tabular}

Fonte: (JAHANSHAHI, 2013) 


\section{8. "Modelo de Jahanshahi" para um Sistema "Poço-Pipeline- Riser"}

Na seção 3.7, analisou-se um sistema com vazões de entrada constantes, definidas na seção 3.7.2 (condições de contorno de entrada). Porém, para uma análise mais realista de um sistema de produção de petróleo, deve-se utilizar uma vazão de entrada variável com a pressão. Portanto adicionou-se ao modelo um poço de petróleo, onde é assumido que a pressão do reservatório é constante (condição de contorno), conforme Figura 3-15.

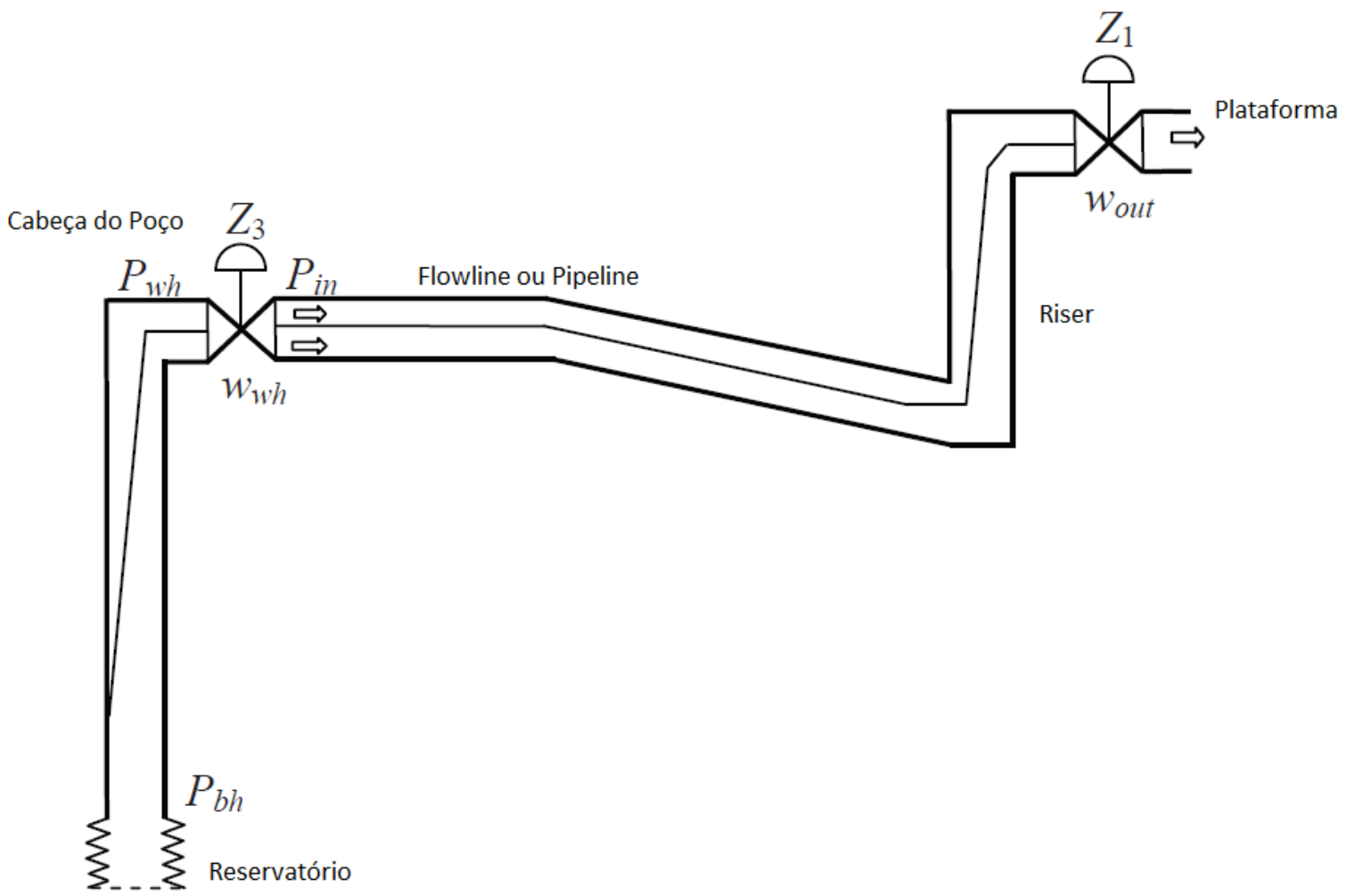

Fonte: (JAHANSHAHI, 2013)

Figura 3-15 - Esquemático de um sistema poço-pipeline-riser.

\subsubsection{Modelo Simplificado Poço-Pipeline-Riser}

Para o modelo poço-pipeline-riser, adicionou-se duas variáveis de estado, a massa do gás e a massa do líquido dentro do poço, resultando em um modelo com seis estados. As equações de estado adicionais são:

$$
\frac{d m_{g, w}}{d t}=\left(\frac{g o r}{g o r+1}\right) \cdot w_{r}-w_{g, w h}
$$




$$
\frac{d m_{l, w}}{d t}=\left(\frac{1}{g o r+1}\right) \cdot w_{r}-w_{l, w h}
$$

onde gor é a razão mássica gás/líquido média produzida pelo reservatório, considerase neste modelo que este parâmetro do poço é conhecido, $w_{g, w h}$ e $w_{l, w h}$ são as vazões de gás e líquido na cabeça do poço. A vazão $w_{r}$ do reservatório para o poço foi considerada como uma relação linear com a pressão:

$$
w_{r}=P I \cdot \max \left(0, P_{r e s}-P_{b h}\right)
$$

Sendo $P I$, o índice de produtividade do poço, $P_{\text {res }}$ a pressão do reservatório que pode ser considerada constante para um período pequeno de análise (alguns dias)

A pressão $P_{b h}$ no fundo do poço é:

$$
P_{b h}=P_{w h}+\bar{\rho}_{m, w} \cdot g \cdot L_{w}+\Delta P_{f w}
$$

onde $\Delta P_{f w}$, a perda de carga no poço devido ao atrito, é dada por:

$$
\Delta P_{f w}=\frac{\bar{\alpha}_{l, w} \cdot \lambda_{w, \bar{l}} \cdot \bar{U}_{s l, w}^{2} \cdot L_{w}}{2 \cdot D_{w}}
$$

Aqui a fração volumétrica média de líquido no poço é dada por:

$$
\bar{\alpha}_{l, w}=\frac{m_{l, w}}{V_{w} \cdot \rho_{l}}
$$

Para o fator de atrito no poço, é utilizada a mesma correlação do pipeline:

$$
\frac{1}{\sqrt{\lambda_{w}}}=-1,8 \cdot \log _{10} \cdot\left[\left(\frac{\epsilon / D_{w}}{3,7}\right)^{1,11}+\frac{6,9}{R e_{w}}\right]
$$

Tem-se o número de Reynolds para o escoamento no poço:

$$
R e_{w}=\frac{\rho_{l} \cdot \overline{\bar{U}}_{s l, w} \cdot D_{w}}{\mu}
$$

E a velocidade superficial média de líquido no poço é:

$$
\bar{U}_{s l, w}=\frac{4 \cdot \bar{w}_{n o m}}{\pi \cdot D_{w}^{2} \cdot \rho_{l}}
$$

onde $\bar{w}_{\text {nom }}$ é a vazão nominal do poço, conhecida a priori.

Como outras variáveis importantes do modelo do poço, a densidade média da mistura bifásica é:

$$
\bar{\rho}_{m, w}=\frac{m_{g, w}+m_{l, w}}{V_{w}}
$$

A densidade da fase gás é: 


$$
\rho_{g, w}=\frac{m_{g, w}}{V_{w}-m_{l, w} / \rho_{l}}
$$

E a pressão na cabeça do poço, considerando gás ideal é:

$$
P_{w h}=\frac{m_{g, w} \cdot R \cdot T_{w h}}{M_{g} \cdot\left(V_{w}-m_{l, w} / \rho_{l}\right)}
$$

Para calcular as frações volumétricas no topo do poço, utiliza-se as mesmas considerações feitas para o riser:

$$
\alpha_{l, w t}=2 . \bar{\alpha}_{l, w}-\alpha_{l, w b}
$$

Neste caso, assume-se que o fluido do reservatório está saturado e a fração volumétrica do líquido é $\alpha_{l, w b}=1$, pois a pressão no fundo do poço é bastante elevada, mantendo todo o gás em solução. A fração mássica do gás no topo do poço é dada por:

$$
\alpha_{g, w t}^{m}=\frac{\left(1-\alpha_{l, w t}\right) \cdot \rho_{g, w}}{\alpha_{l, w t} \cdot \rho_{l}+\left(1-\alpha_{l, w t}\right) \cdot \rho_{g, w}}
$$

E a densidade da mistura no topo do poço é:

$$
\rho_{w t}=\alpha_{l, w t} \cdot \rho_{l}+\left(1-\alpha_{l, w t}\right) \cdot \rho_{g, w}
$$

onde $P_{i n}$ é a pressão na entrada do pipeline.

As vazões das fases gás e líquido na cabeça do poço são dadas por:

$$
\begin{aligned}
& w_{g, w h}=w_{w h} \cdot \alpha_{g, w t}^{m} \\
& w_{l, w h}=w_{w h .} \cdot\left(1-\alpha_{g, w t}^{m}\right)
\end{aligned}
$$

As vazões de gás e líquido no pipeline são dadas respectivamente por:

$$
\begin{aligned}
& w_{g, i n}=w_{g, w h}+d_{1} \\
& w_{l, i n}=w_{l, w h}+d_{2}
\end{aligned}
$$

onde $d_{1}$ e $d_{2}$ podem ser consideradas como distúrbios de outros poços na malha de drenagem. 
Tabela 3 - Parâmetros adicionais para o sistema poço-pipeline-riser

\begin{tabular}{cccc}
\hline Símbolo & Descrição & Valor & Unidades \\
\hline$P_{\text {res }}$ & Pressão do reservatório & 320 & $\mathrm{bar}$ \\
$P I$ & Índice de produtividade & $2,75 \times 10-6$ & $\mathrm{~kg} /(\mathrm{s} . P a)$ \\
$w_{\text {nom }}$ & Vazão mássica nominal do reservatório & 9 & $\mathrm{~kg} / \mathrm{s}$ \\
$g o r$ & Razão mássica gás óleo & 0,0417 & - \\
$T_{w}$ & Temperatura do poço & 369 & $\mathrm{~K}$ \\
$D_{w}$ & Diâmetro do poço & 0,12 & $\mathrm{~m}$ \\
$L_{w}$ & Profundidade do poço & 3000 & $\mathrm{~m}$ \\
\hline & Fonte: (JAHANSHAHI, 2013) & &
\end{tabular}

\subsection{Modelagem do sistema no simulador OLGA ${ }^{\circledR}$}

\subsubsection{Modelagem do Sistema Pipeline-Riser}

O sistema pipeline-riser descrito na seção 3.7 foi modelado no software OLGA ${ }^{\circledast}$ considerando os parâmetros geométricos informados na Tabela 2.

Para este modelo, considerou-se o comprimento total do trecho pipeline de $4300 \mathrm{~m}$, sendo este, formado por um trecho horizontal de $2000 \mathrm{~m}$ (PIPE-1) e por um trecho com inclinação descendente de 1 grau, composto por uma seção de 2000m (PIPE-2) e outra de $300 \mathrm{~m}$ (PIPE-3).

O trecho riser foi composto por um duto de $300 \mathrm{~m}$ com inclinação vertical (PIPE-4) e um trecho horizontal de 100m (PIPE-5), a válvula choke está situada ao final do trecho horizontal, conectada a montante de um separador de produção com pressão constante de 50,1 bar. Os trechos denominados PIPE-1 a PIPE-5 fazem parte da nomenclatura constante na Tabela 4.

Para todo o duto, a rugosidade considerada foi de $0,028 \mathrm{~mm}$. 


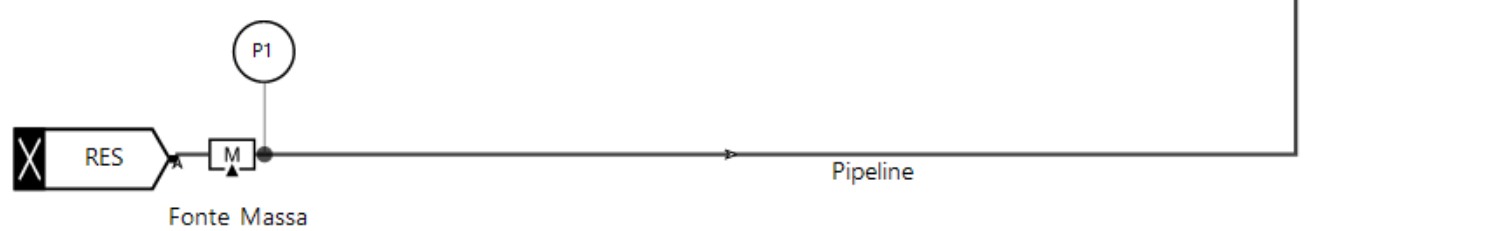

Figura 3-16 - Modelo do OLGA ${ }^{\circledR}$ para o sistema pipeline-riser

Tabela 4 - Dados da geometria do sistema pipeline-riser modelado no OLGA ${ }^{\circledR}$

\begin{tabular}{cccccc}
\hline Trecho & Nome & Diâmetro & Rugosidade & Comprimento & Elevação \\
\hline Pipeline-Riser & PIPE-1 & $0.12 \mathrm{~m}$ & $2.8 \mathrm{E}-05 \mathrm{~m}$ & $2000 \mathrm{~m}$ & $0 \mathrm{~m}$ \\
Pipeline-Riser & PIPE-2 & $0.12 \mathrm{~m}$ & $2.8 \mathrm{E}-05 \mathrm{~m}$ & $2000 \mathrm{~m}$ & $-34.9 \mathrm{~m}$ \\
Pipeline-Riser & PIPE-3 & $0.12 \mathrm{~m}$ & $2.8 \mathrm{E}-05 \mathrm{~m}$ & $300 \mathrm{~m}$ & $-5.24 \mathrm{~m}$ \\
Pipeline-Riser & PIPE-4 & $0.1 \mathrm{~m}$ & $2.8 \mathrm{E}-05 \mathrm{~m}$ & $300 \mathrm{~m}$ & $300 \mathrm{~m}$ \\
Pipeline-Riser & PIPE-5 & $0.1 \mathrm{~m}$ & $2.8 \mathrm{E}-05 \mathrm{~m}$ & $100 \mathrm{~m}$ & $0 \mathrm{~m}$ \\
\hline
\end{tabular}

Neste problema, considerou-se a fonte de massa com vazão de $9 \mathrm{~kg} / \mathrm{s}$ e temperatura da mistura de $293,15 \mathrm{~K}\left(20^{\circ} \mathrm{C}\right)$. Pela curva mostrada na Figura 3-17 é possível obter a fração mássica do gás na entrada do pipeline. Para $20^{\circ} \mathrm{C}$, tem-se que $4 \%$ da vazão mássica é relativa a fração gasosa, logo, as vazões mássicas de alimentação do duto são de $0,36 \mathrm{~kg} / \mathrm{s}$ na fase gasosa e $8,64 \mathrm{~kg} / \mathrm{s}$ na fase líquida. 


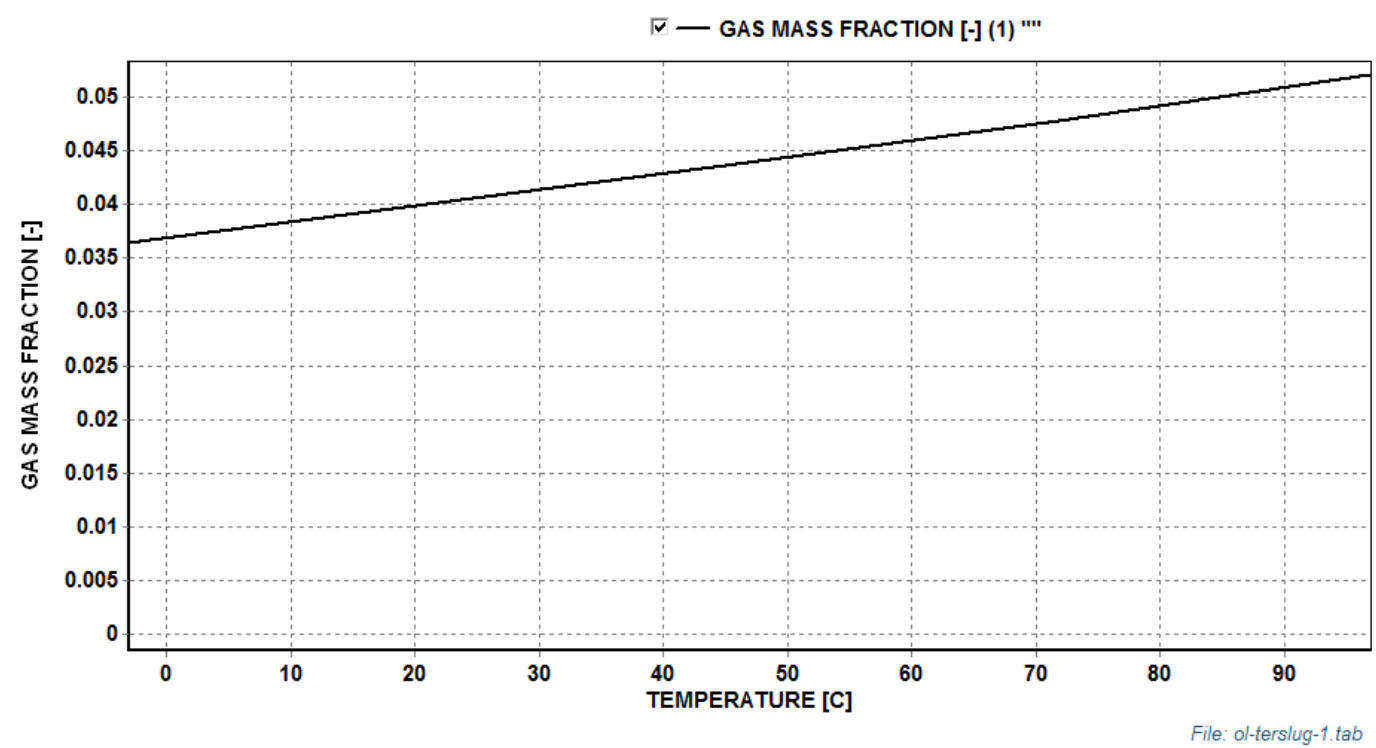

Figura 3-17 - Curva de fração mássica de gás $x$ temperatura do fluido

\subsubsection{Modelagem do Sistema Poço-Pipeline-Riser}

O sistema poço-pipeline-riser descrito na seção 3.8 foi modelado no software OLGA ${ }^{\circledR}$ considerando os parâmetros geométricos informados na Tabela 3.

Para este modelo, considerou-se o comprimento do poço de $3000 \mathrm{~m}$ vertical, pressão de reservatório de 320 bar e temperatura do fluido a 369K. O modelo do reservatório foi considerado linear, ou seja, a vazão de produção varia linearmente ao diferencial de pressão imposto ao reservatório. 


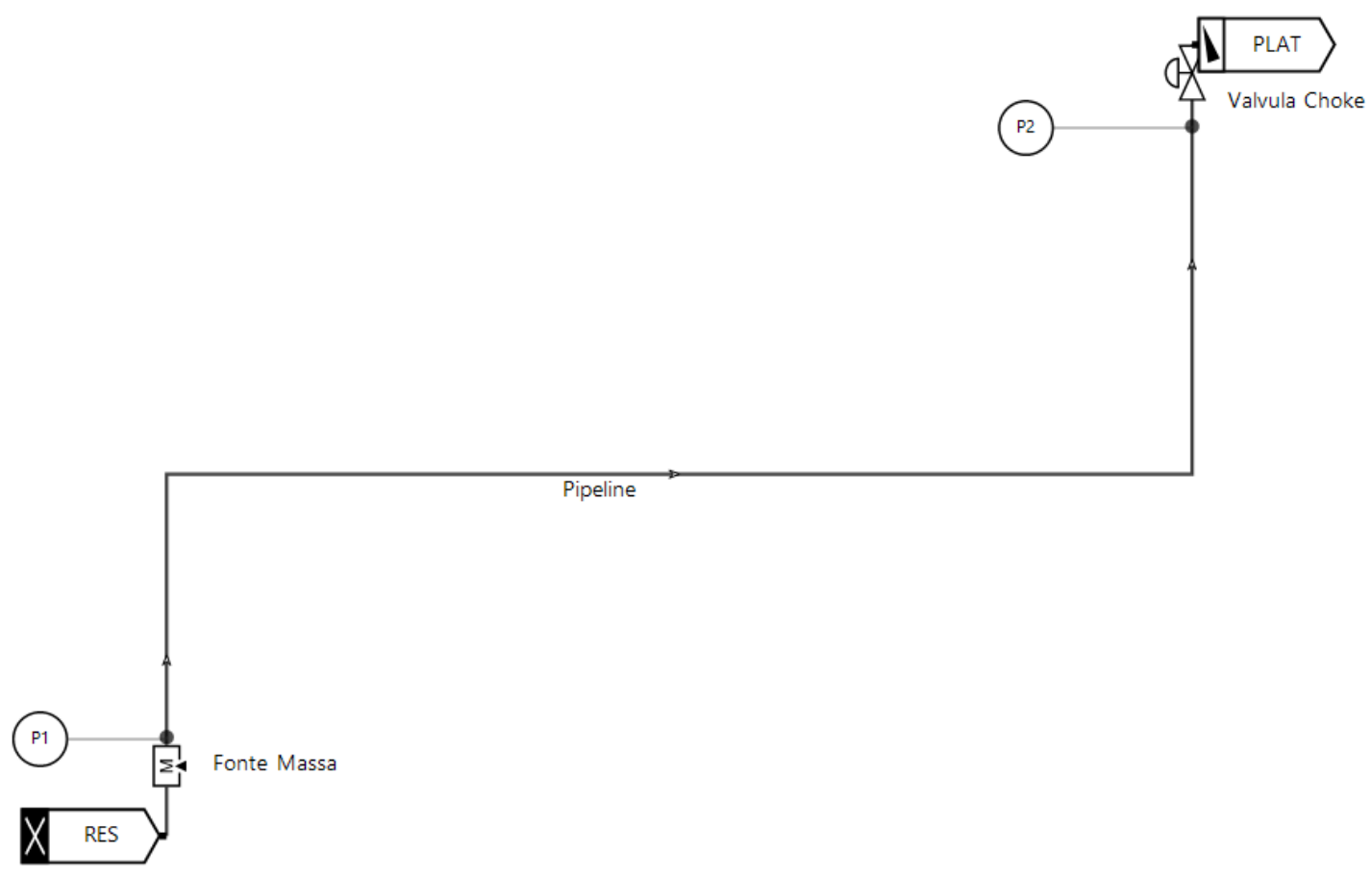

Figura 3-18 - Modelo do OLGA ${ }^{\circledR}$ para o sistema poço-pipeline-riser

Tabela 5 - Dados da geometria do sistema poço-pipeline-riser modelado no OLGA ${ }^{\circledR}$

\begin{tabular}{cccccc}
\hline Trecho & Nome & Diâmetro & Rugosidade & Comprimento & Elevação \\
\hline Poço & PIPE-1 & $0.12 \mathrm{~m}$ & $2.8 \mathrm{E}-05 \mathrm{~m}$ & $3000 \mathrm{~m}$ & $3000 \mathrm{~m}$
\end{tabular}




\section{RESULTADOS E DISCUSSÃO}

\subsection{Modelo do sistema pipeline-riser}

\subsubsection{Pressões de Entrada e Saída e Diagrama de Bifurcação do Pipeline- Riser no OLGA ${ }^{\circledR}$}

A partir do sistema pipeline-riser modelado no OLGA ${ }^{\circledR}$ na seção 3.9.1, simulou-se o comportamento das pressões de entrada (pipeline) e saída (topo do riser) para uma abertura da válvula choke em rampa (entre $4 \%$ e 10\%). O objetivo é determinar a abertura crítica da válvula, na qual as pressões da entrada do pipeline e do topo do riser se tornam instáveis e iniciam o comportamento oscilatório. Na Figura 4-1, a abertura da válvula choke está mostrada na escala de 0.04-0.10 no lado direito e as pressões na entrada do pipeline e no topo do riser estão mostradas na escala 50-80 no lado esquerdo do gráfico. Através da análise do gráfico, nota-se que as oscilações nas pressões na entrada do pipeline e no topo dor riser se iniciam para aberturas da válvula choke em $7 \%$, sendo esta, a abertura crítica em que o sistema se torna oscilatório.

Pressões na entrada do pipeline e no topo do riser para uma abertura crescente da válvula choke

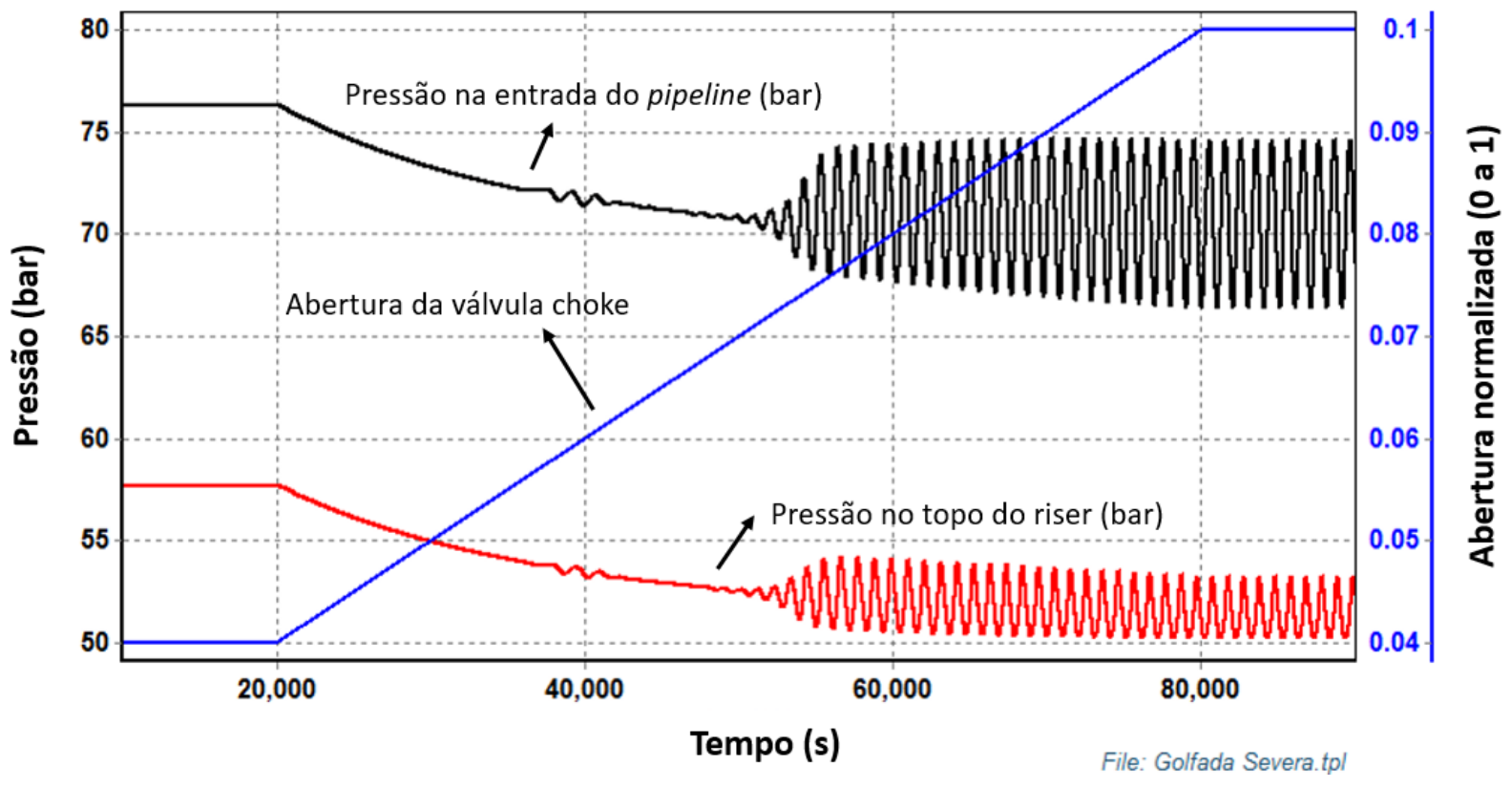

Figura 4-1 - Comportamento das pressões na entrada do pipeline (PIPE-1) e no topo do riser (PIPE-5) para uma abertura crescente da válvula choke 
A mesma simulação foi realizada, agora com uma maior variação da válvula choke ( $2 \%$ a $100 \%$ ), com o objetivo de visualizar os ranges de oscilação das pressões para cada abertura da válvula choke e determinar de forma indireta, o diagrama de bifurcação do sistema, estes resultados podem ser visualizados na Figura 4-2. O diagrama de bifurcação é formado pelos valores de máximo e mínimo das pressões na entrada do pipeline e no topo do riser. A abertura da válvula choke está mostrada na escala de 0.0-1.0 no lado direito e as pressões na entrada do pipeline e no topo do riser estão mostradas na escala 50-100 no lado esquerdo do gráfico.

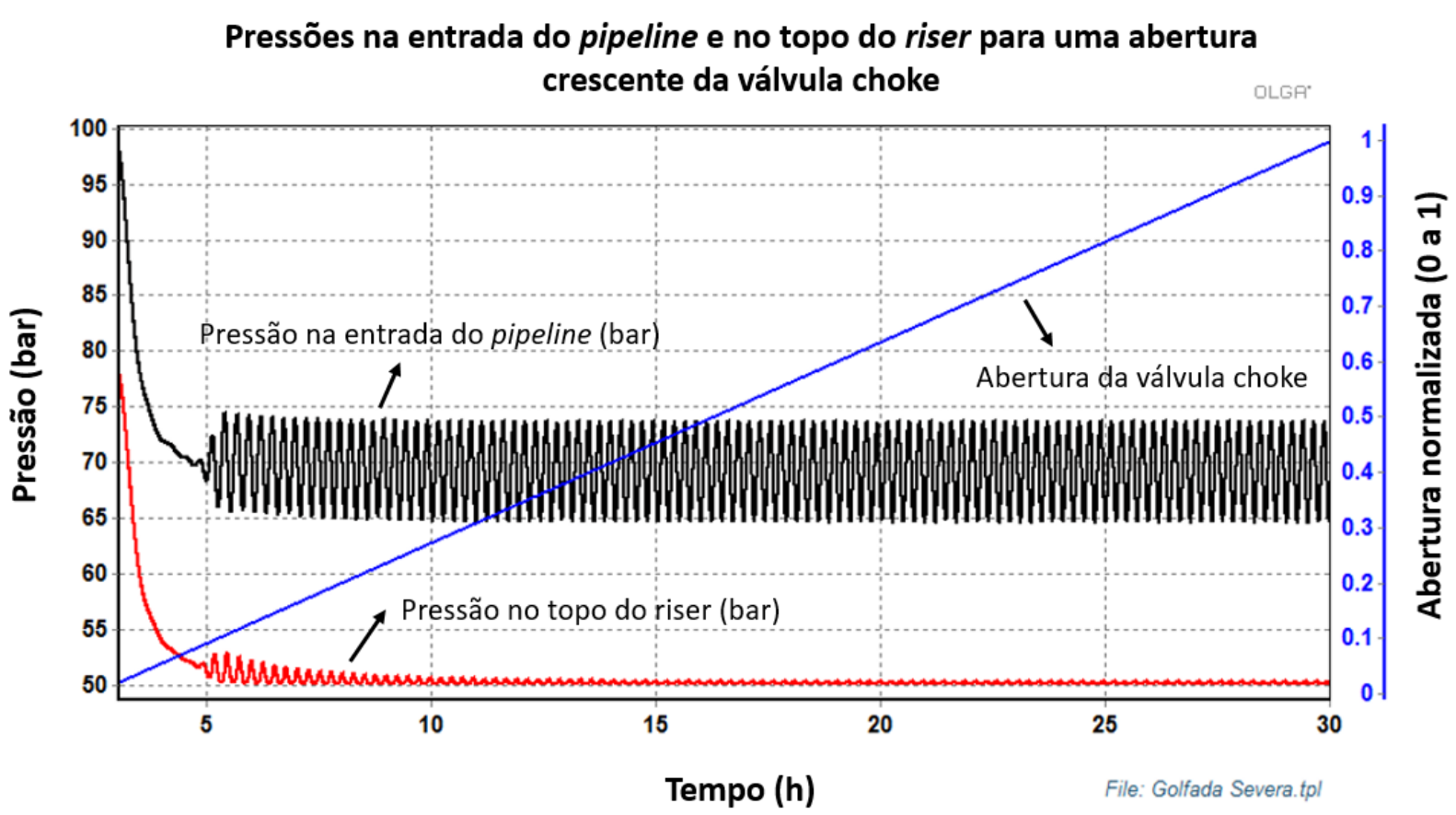

Figura 4-2 - Oscilação das pressões de entrada do pipeline e de topo do riser para diferentes aberturas da válvula choke

\subsubsection{Diagramas de Bifurcação do Modelo Pipeline-Riser no MATLAB}

Os diagramas de bifurcação obtidos no MATLAB através do modelo simplificado de Jahanshahi para o sistema pipeline-riser são apresentados na Figura 4-3. Diagrama de bifurcação é um método adequado para avaliar o ponto em que o sistema se torna instável. Para presente caso, o modelo se torna instável para aberturas da válvula choke superiores a $7 \%$, ponto em que ocorre a separação das linhas representativas das pressões máximas e mínimas, tanto para a pressão de entrada do pipeline (Figura 4-3), quanto para a pressão do topo do riser (Figura 4-4), onde a linha tracejada 
representa a região de equilíbrio instável, representando o ponto ideal de operação, onde é possível elevar a produtividade do poço.

A avaliação do modelo pipeline-riser pode ser realizada, comparando-se as oscilações de pressões de entrada do pipeline e de topo do riser obtidas no OLGA ${ }^{\circledR}$ e mostradas na Figura 4-2, com os diagramas de bifurcação obtidos via MATLAB mostrados na Figura 4-3 e na Figura 4-4. Tanto o diagrama obtido pelo OLGA ${ }^{\circledR}$ quanto no MATLAB são mapas de bifurcação de Hopf (STORKAAS; SKOGESTAD, 2007) e (ZAKARIAN, 2000)

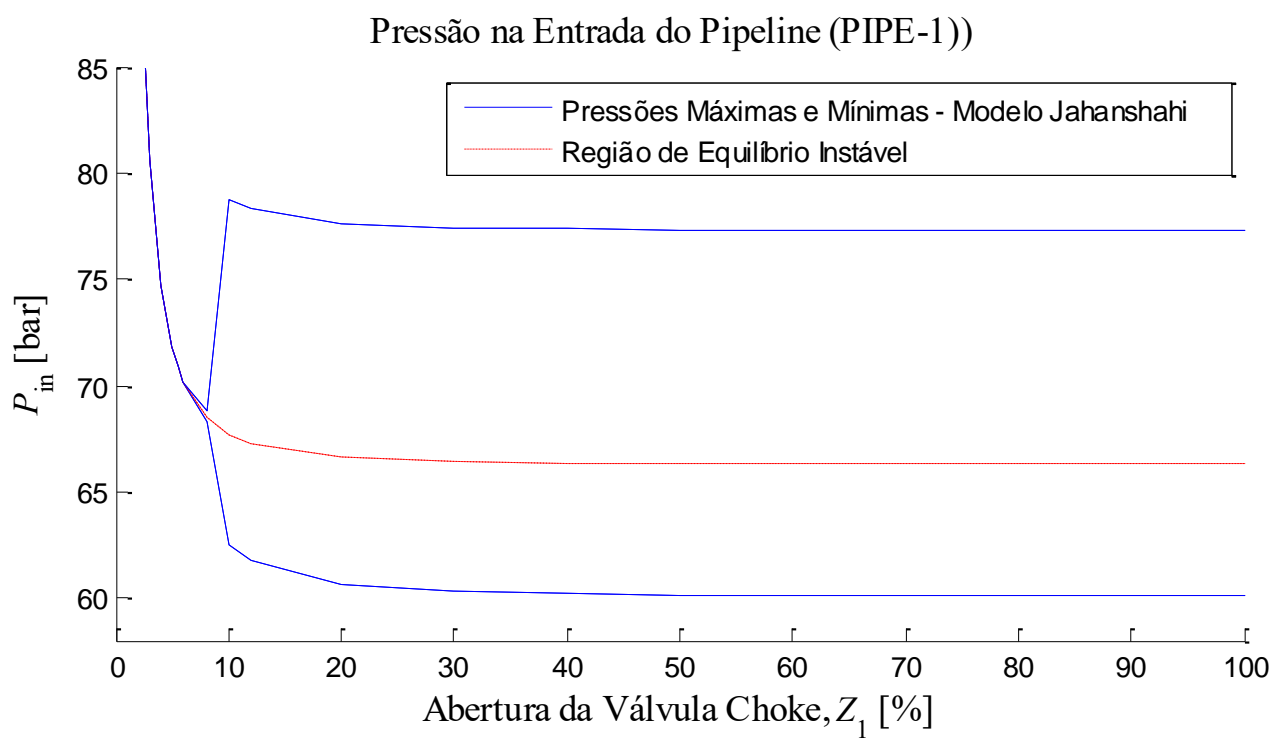

Figura 4-3 - Diagrama de bifurcação - pressão na entrada do pipeline x abertura da válvula choke

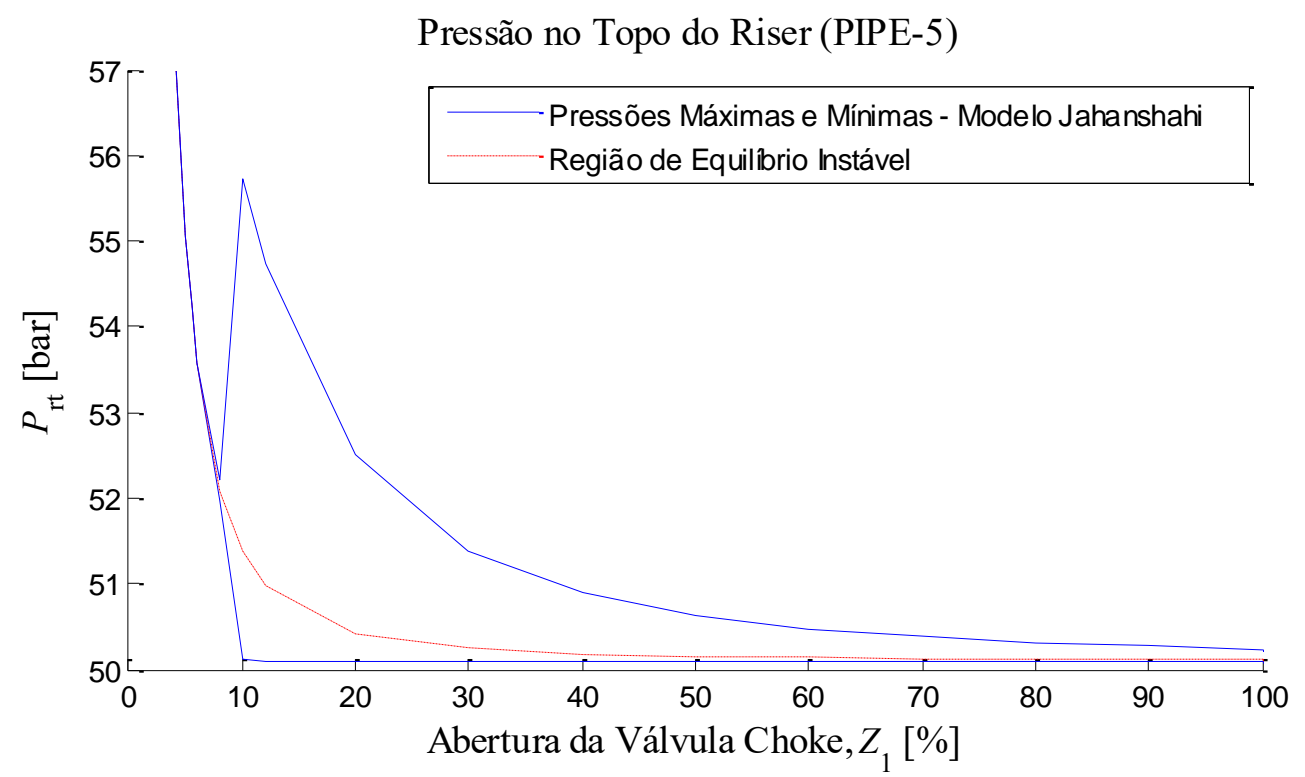

Figura 4-4 - Diagrama de bifurcação - pressão no topo do riser $\mathrm{x}$ abertura da válvula choke 
Portanto, é possível comprovar que, para ambos os casos de MATLAB e OLGA ${ }^{\circledR}$, as pressões de entrada do pipeline e do topo do riser, iniciam o comportamento oscilatório para aberturas da válvula choke de aproximadamente $7 \%$.

Nota-se também que, para as simulações do MATLAB, os ranges de oscilação das pressões na entrada do pipeline (entre 64 bar e 78 bar) e no topo do riser (entre 50 bar e 56 bar) são maiores que os obtidos pelo OLGA ${ }^{\circledR}$ (respectivamente entre 65 bar e 74 bar e entre 50 bar e 53 bar). Apesar das diferenças verificadas, considera-se que o modelo de Jahanshahi implementado no MATLAB apresenta bom ajuste com os resultados das simulações no OLGA ${ }^{\circledR}$.

\subsection{Modelo do Sistema Poço-Pipeline-Riser}

\subsubsection{Pressões de Entrada e Saída e Diagrama de Bifurcação do Poço- Pipeline-Riser no OLGA ${ }^{\circledR}$}

A mesma análise realizada para o sistema pipeline-riser foi repetida para o sistema poço-pipeline-riser. Os resultados podem ser visualizados na Figura 4-5, onde estão mostradas as faixas de oscilação das pressões para cada abertura da válvula choke, sendo possível visualizar o diagrama de bifurcação do sistema poço-pipeline-riser, similar ao item anterior, formado pelos valores de máximo e mínimo das pressões na entrada do pipeline e no topo do riser mostradas respectivamente nas escalas $50-85$ para a pressão de topo do riser e 240-300 para a pressão do fundo do poço no lado esquerdo da Figura 4-5. A abertura da válvula choke está mostrada na escala de 0.01.0 no lado direito da Figura 4-5. 
Pressões no fundo do poço e no topo do riser para uma abertura crescente da válvula choke

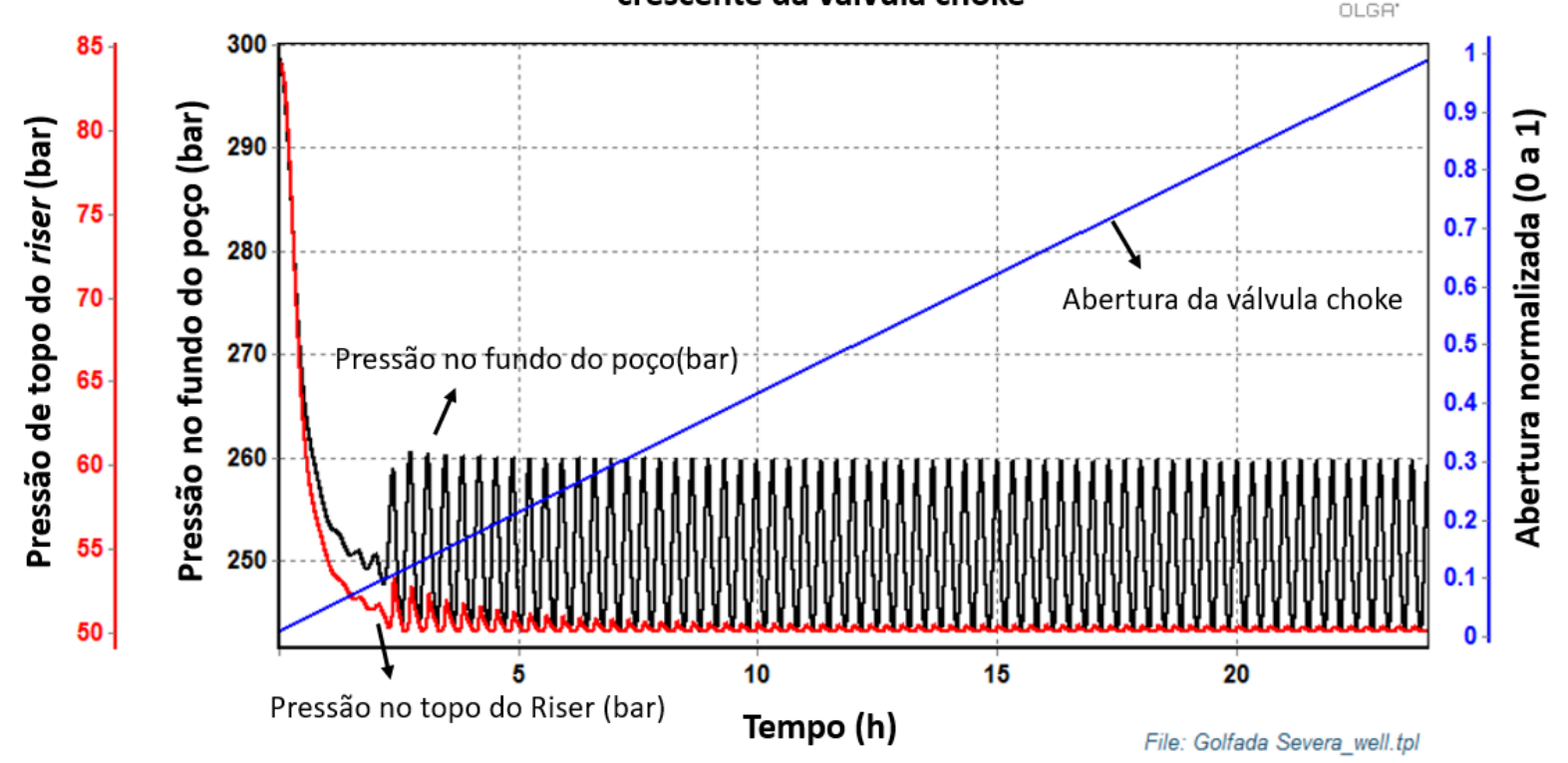

Figura 4-5 - Oscilação das pressões do fundo do poço e no topo do riser para diferentes aberturas da válvula choke

\subsubsection{Diagramas de Bifurcação pelo Modelo Poço-Pipeline-Riser no MATLAB}

De forma análoga a realizada para o sistema pipeline-riser na seção 4.1.2, foram ilustrados os diagramas de bifurcação obtidos no MATLAB, através do modelo simplificado de Jahanshahi para o sistema poço-pipeline-riser. Os diagramas de bifurcação mostrados na Figura 4-6 e na Figura 4-7 podem ser comparados com a simulação do OLGA ${ }^{\circledR}$ mostrada na Figura 4-5. 


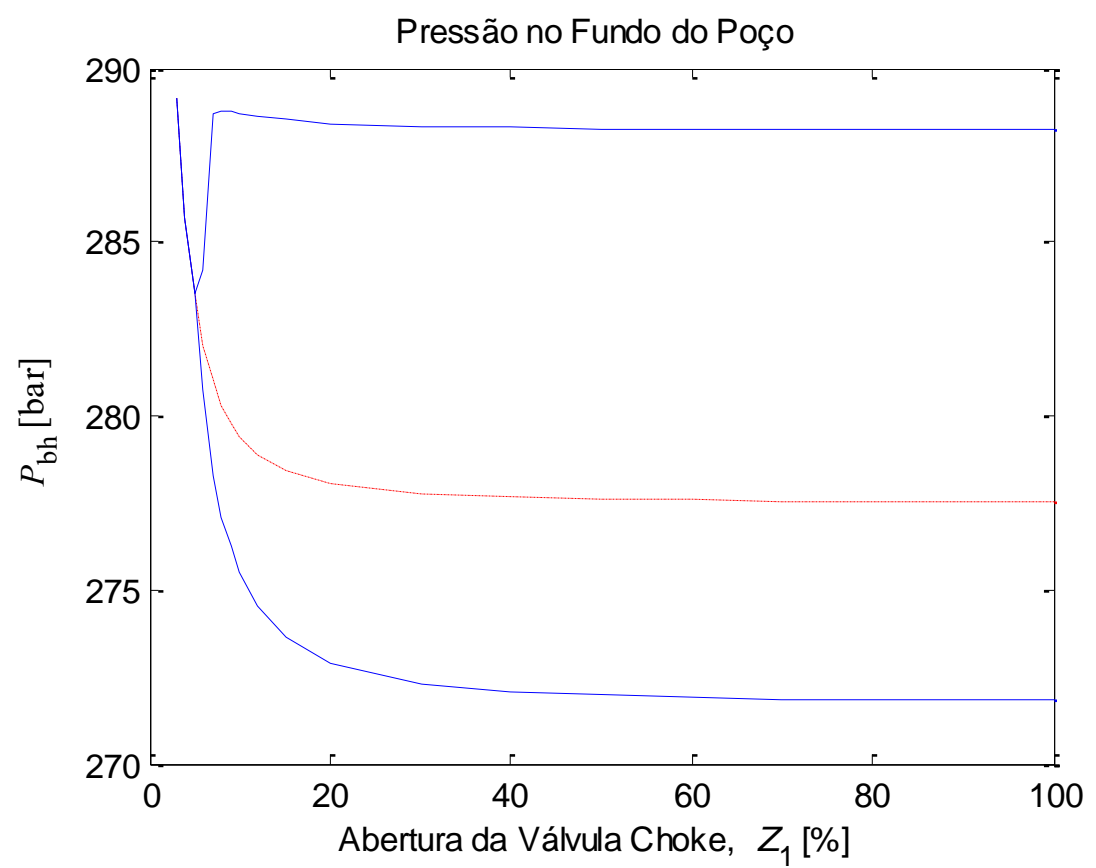

Figura 4-6 - Diagrama de bifurcação - pressão no fundo do poço x abertura da válvula choke

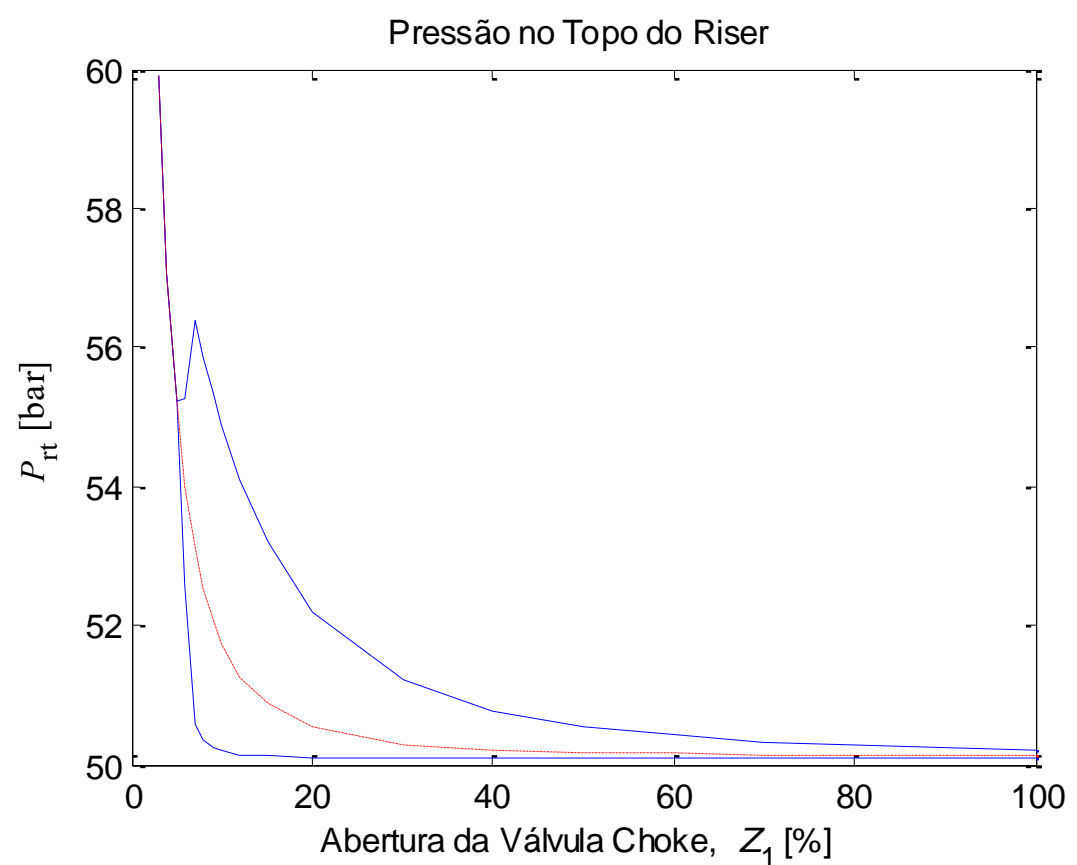

Figura 4-7 - Diagrama de bifurcação - pressão no topo do riser $\mathrm{x}$ abertura da válvula choke

Analogamente, é possível avaliar o modelo poço-pipeline-riser, comparando-se a Figura 4-5 que representa os resultados do OLGA ${ }^{\circledR}$ com a Figura 4-6 e a Figura 4-7, que representam os diagramas de bifurcação obtidos via MATLAB. Novamente, os resultados obtidos pelo OLGA ${ }^{\circledR}$ apresentam ranges menores que os simulados pelo MATLAB. 
Apesar das diferenças verificadas, considera-se que o modelo de Jahanshahi implementado no MATLAB apresenta bom ajuste com os resultados das simulações no OLGA ${ }^{\circledR}$.

Com base em tais resultados, utilizou-se o modelo simplificado modelado no MATLAB para o desenvolvimento de algoritmos de controle, com o objetivo de suprimir golfadas nos pontos de operação em que o sistema é instável (para aberturas da válvula choke maiores que a abertura crítica).

\subsection{Algoritmos de Controle para Mitigação de Golfadas}

Diversos algoritmos de controle têm sido desenvolvidos com o intuito de atenuar os problemas gerados pela produção em golfadas. O sistema proposto é instável para aberturas fixas da válvula choke superiores a abertura crítica, portanto, para se operar na região instável, aumentando a produtividade do poço e mitigando as golfadas, é necessário modular adequadamente a válvula. Para isto, diversos algoritmos de controle foram propostos (JAHANSHAHI; SKOGESTAD, 2013), (JAHANSHAHI et al., 2014), (JAHANSHAHI; SKOGESTAD, 2015), (OLIVEIRA; JÄSCHKE; SKOGESTAD, 2015), (SIVERTSEN; SKOGESTAD, 2005) e (ZHOU; KAASA; AAMO, 2008).

Como variável controlada, são utilizadas preferencialmente as pressões de fundo do poço ou de topo do riser, pois as mesmas são as mais comumente encontradas nos sistemas reais, seja através de sensores de pressão submarinos ou na plataforma. Porém, os transmissores submarinos possuem como desvantagem, uma manutenção de alto custo em caso de falha.

Como variável manipulada, é utilizada a abertura da válvula choke no topo do riser, visto que, apesar de ser possível a existência da válvula choke em outras posições, como na cabeça do poço ou na base do riser, estas não são comuns em poços produtores de óleo. 
Neste trabalho são adotadas duas abordagens distintas para as técnicas de controle, a primeira será a utilização de um controlador PI adaptativo que consiga atuar perante as mudanças inerentes do sistema. A segunda abordagem é a utilização de um Regulador Linear Quadrático associado a um observador de estados com filtro de Kalman Unscented. O objetivo é verificar como o sistema se comporta em caso de utilização de um observador para compensar a falha de um sensor submarino.

\subsubsection{PI Adaptativo}

Os sistemas de controle aplicados na produção de petróleo se tornam instáveis após algum tempo, devido, por exemplo, à depleção natural do reservatório, reduzindo a produtividade do poço. Para compensar esta deficiência, são necessários controladores que consigam operar em diferentes pontos de operação e que lidem adequadamente com as não linearidades características deste tipo de sistema.

Para o controlador PI com ganho adaptativo, foi utilizado um algoritmo encontrado na literatura (JAHANSHAHI, 2013), que é descrito em detalhes abaixo.

A implementação deste controlador é realizada com base no ganho estático do sistema e na abertura média da válvula choke. No diagrama de bifurcação, a linha central representa a região desejada de operação conforme já citado nas seções anteriores. Esta linha está diretamente relacionada as propriedades da válvula choke (JAHANSHAHI, 2013), a equação da válvula possui a seguinte definição:

$$
w=C_{v} \cdot f(z) \cdot \sqrt{\rho \cdot \Delta P}
$$

onde $w(\mathrm{~kg} / \mathrm{s})$ representa a vazão mássica de saída da válvula, $\Delta P$ representa a perda de carga através da válvula e $C_{v}$ é uma constante conhecida da válvula. Logo, a perda de carga pode ser reescrita como:

$$
\Delta P=\frac{\bar{\alpha}}{f(z)^{2}}
$$

Onde $\bar{\alpha}$ é uma constante e pode ser definida por:

$$
\bar{\alpha}=\frac{1}{\bar{\rho}} \cdot\left(\frac{\bar{w}}{C_{v}}\right)^{2}
$$

Sendo $\bar{w}$ e $\bar{\rho}$ a vazão mássica média de saída e a densidade média da mistura respectivamente, que podem ser obtidos das seguintes equações: 


$$
\begin{aligned}
& \bar{w} \cong w_{g, \text { in }}+w_{l, i n} \\
& \bar{\rho}=\bar{\alpha}_{l} \cdot \rho_{l}+\left(1-\bar{\alpha}_{l}\right) \cdot \bar{\rho}_{g}
\end{aligned}
$$

Portanto, pela equação 4.2, a pressão a montante do choke é dada por:

$$
P_{\text {in }}=\frac{\bar{\alpha}}{f(z)^{2}}+\bar{P}_{f o}
$$

onde $\bar{P}_{f o}$, também uma constante, é dada por:

$$
\bar{P}_{f o}=P_{i n}^{*}-\frac{\bar{\alpha}}{f\left(z^{*}\right)^{2}}
$$

e $z^{*}$ é a abertura crítica da válvula choke do diagrama de bifurcação.

Derivando a equação 4.7 em relação a $z$, tem-se o ganho estático:

$$
k(z)=\frac{-2 \bar{a} \cdot \frac{\partial f(z)}{z}}{f(z)^{3}}
$$

Assumindo uma válvula com abertura linear, a equação acima pode ser reescrita:

$$
k(z)=\frac{-2 \bar{a}}{z^{3}}
$$

onde $z$ é a abertura da válvula choke e varia de $0 \leq z \leq 1$.

Os valores do ganho proporcional e do tempo integral do controlador PI podem ser escritos em função da abertura $z$ da válvula choke:

$$
\begin{aligned}
& k_{c}(z)=\frac{\beta \cdot T_{o s c}}{k(z) \cdot \sqrt{z / z^{*}}} \\
& \tau_{I}(z)=3 \cdot T_{o s c} \cdot\left(\frac{z}{z^{*}}\right)
\end{aligned}
$$

onde $T_{\text {osc }}$ representa o período das oscilações no sistema em malha aberta, $z^{*}$ representa a abertura crítica da válvula (mostrada no diagrama de bifurcação) e $\beta$ é uma constante calculada a partir da resposta em degrau do sistema em malha fechada.

Primeiramente são mostrados os gráficos do sistema em malha aberta, das pressões $P 1$ (entrada do pipeline) e $P 2$ (topo do riser), vazão volumétrica $Q$ e vazão mássica $W$ para diferentes aberturas da válvula choke.

A Figura 4-8 mostra as saídas do sistema para uma abertura da válvula choke de 5\% obtidas no MATLAB. Nota-se, conforme seção 5.1 que o sistema é estável para esta 
abertura da válvula choke, mesmo em malha aberta, porém a pressão estabilizada da entrada do pipeline $P 1$ é elevada, aproximadamente de 77,42 bar.
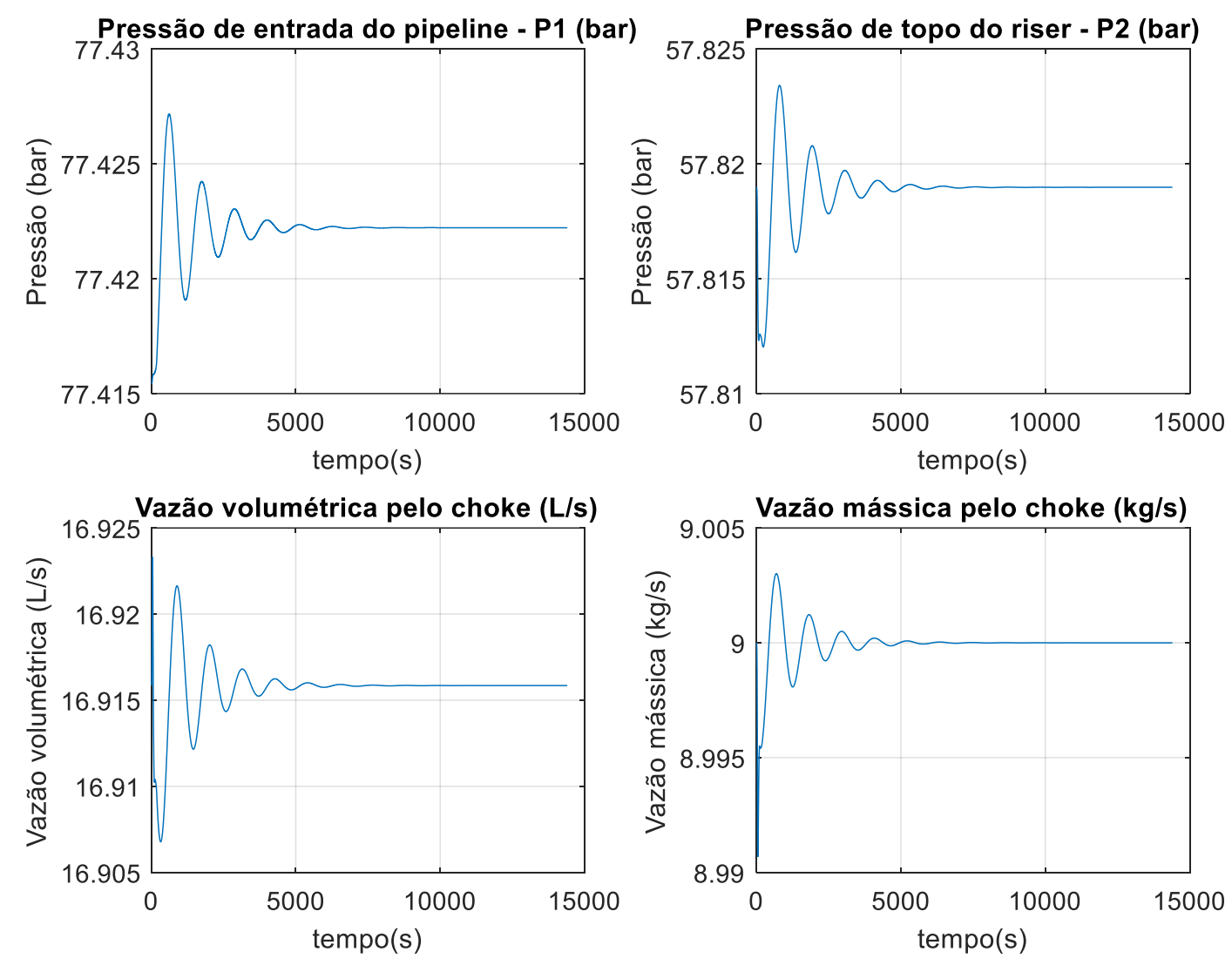

Figura 4-8 - Pressões $P 1$ e $P 2$, vazão volumétrica $Q$ e vazão mássica $W$ para a válvula choke com abertura de $5 \%$

Na Figura 4-9 são mostradas as saídas do sistema para uma abertura da válvula choke de $10 \%$ obtidas no MATLAB. Nota-se que para esta abertura, o sistema já se torna oscilatório em malha aberta, visto que a abertura da válvula choke é maior que a abertura crítica 7\%, valor este, obtido na seção 4.1.1. Apesar do comportamento oscilatório, a pressão média de entrada do pipeline $P 1$ para abertura de $10 \%$ é de 71,56 bar, valor menor que o apresentado pelo sistema para uma abertura da válvula choke de $5 \%$. 

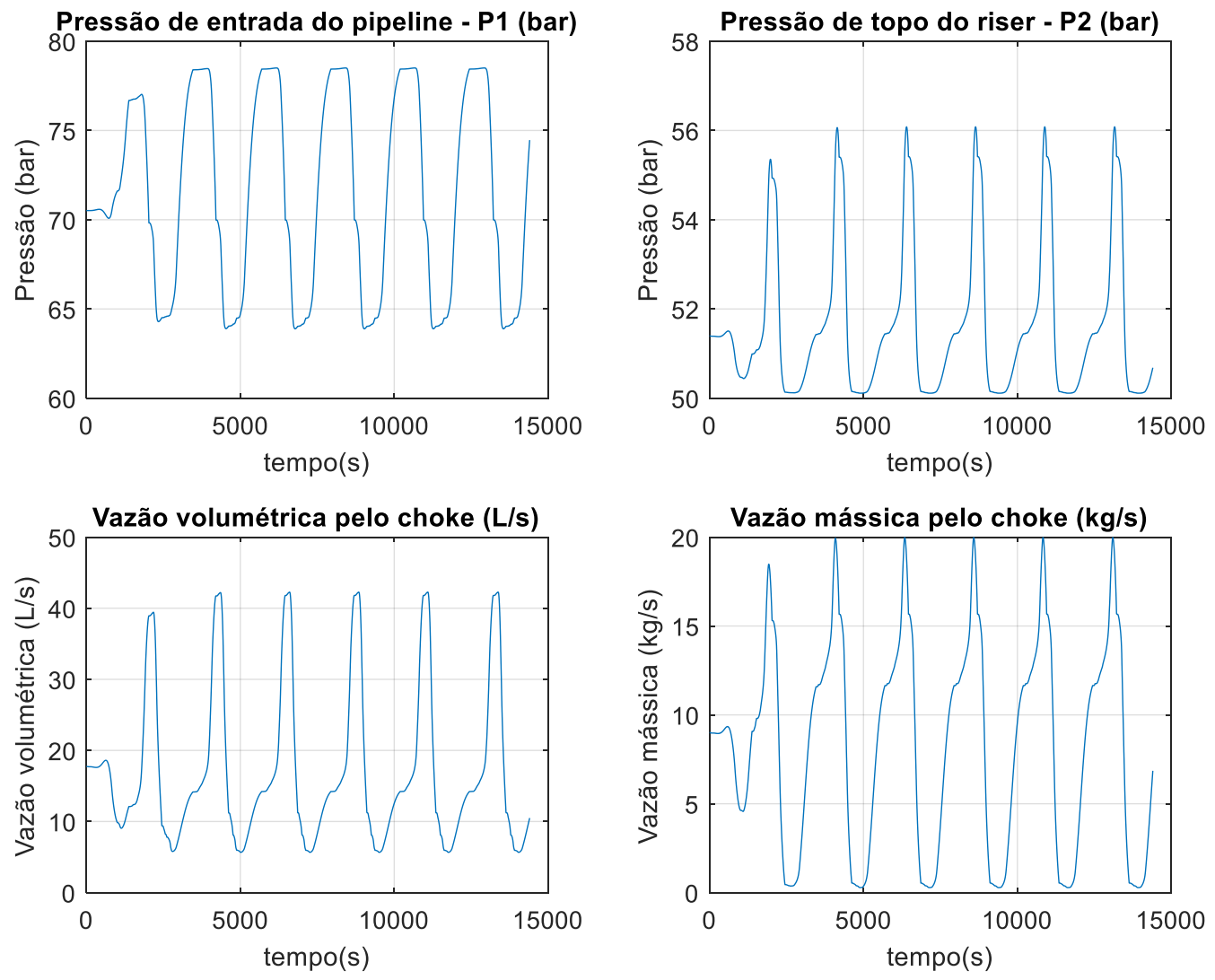

Figura 4-9 - Pressões $P 1$ e $P 2$, vazão volumétrica $Q$ e vazão mássica $W$ para a válvula choke com abertura de $10 \%$

Na Figura 4-10 são mostradas as saídas do sistema para uma abertura da válvula choke de $20 \%$ obtidas no MATLAB. Nota-se que para esta abertura, o sistema continua oscilatório em malha aberta, porém a pressão média de entrada do pipeline Pl é de 69,53 bar, sendo novamente menor que nos casos anteriores. Conforme mostrado na seção 2.3, menores contrapressões impostas ao reservatório resultam em maior produtividade do poço, porém deve-se considerar o comportamento indesejável representado pelas oscilações do poço, resultando em um regime de escoamento por golfadas. 

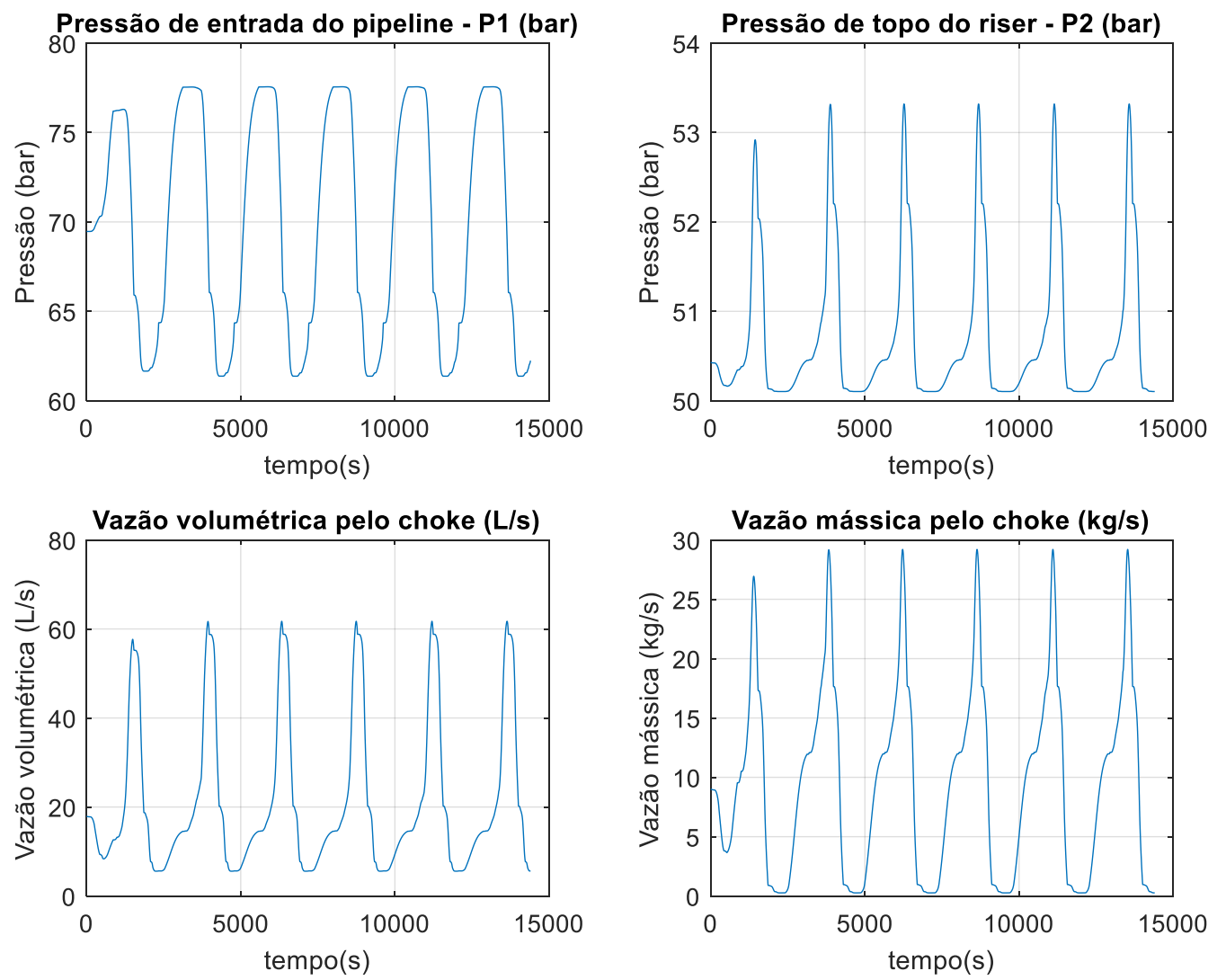

Figura 4-10 - Pressões $P 1$ e $P 2$, vazão volumétrica $Q$ e vazão mássica $W$ para a válvula choke com abertura de $20 \%$

Para comparar a efetividade do controlador PI adaptativo, primeiramente projetou-se um controlador PI de ganho fixo. Linearizou-se o sistema para o ponto de operação de abertura da válvula choke em 10\%, após esta etapa, obteve-se a função de transferência considerando como entrada, a abertura $z$ da válvula choke e como saída, a pressão de entrada do pipeline $P 1$. Obteve-se o ganho proporcional e o tempo integral utilizando a ferramenta "PID Tuner" disponível no MATLAB de forma a adequar o desempenho dinâmico do sistema em malha fechada mostrados na Figura 4-11. Os valores de ganho proporcional e tempo integral obtidos são respectivamente: $k_{p}=-1,09$ e $\tau_{I}=11,9$. 

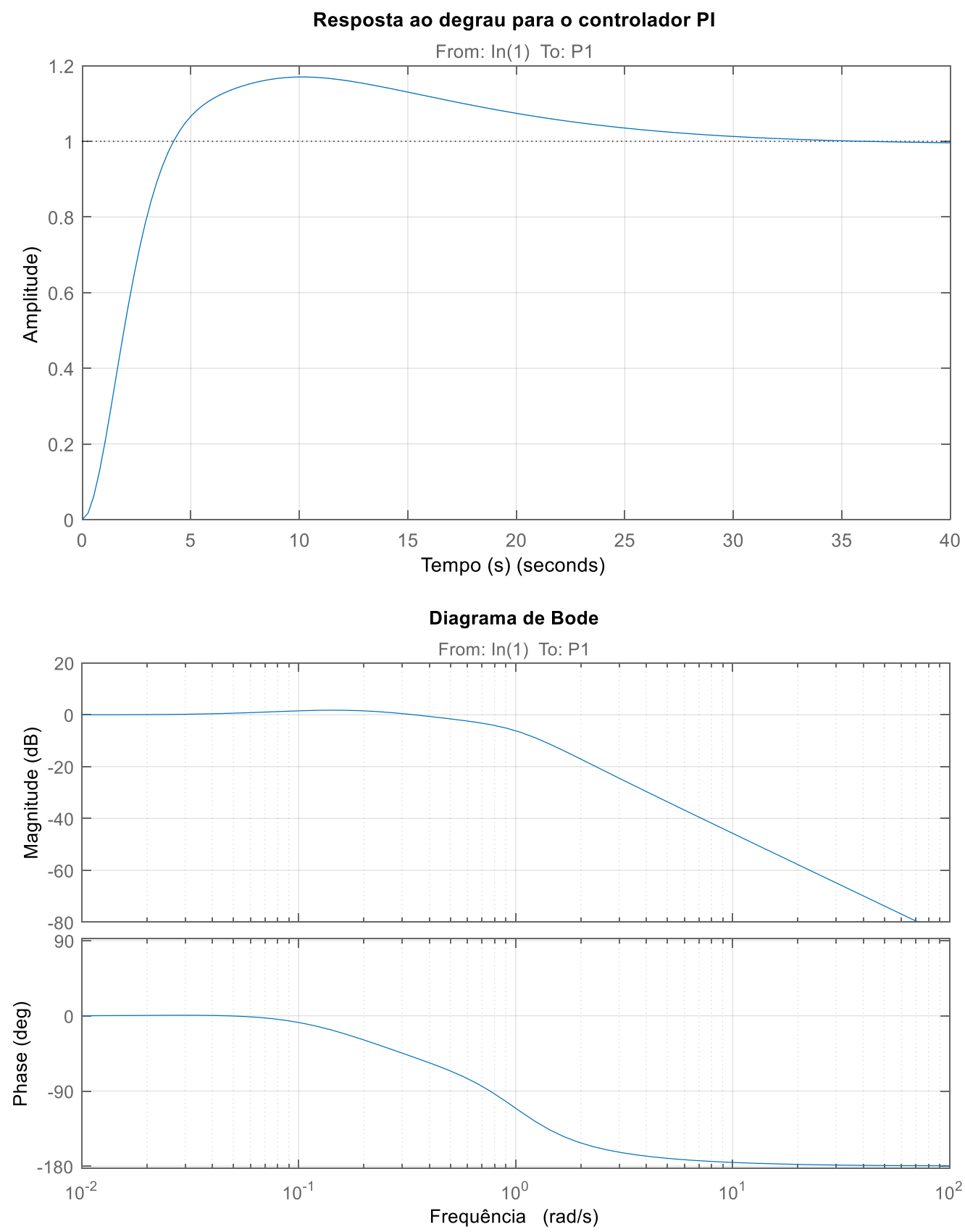

Figura 4-11 - Resposta ao degrau e diagrama de Bode para a função de transferência de $z$ para P1.

A partir do controlador PI de ganho fixo, simulou-se no MATLAB, a resposta em degrau para diferentes aberturas da válvula choke (entre 10\% e 50\%). Nota-se que este controlador só é adequado para aberturas da válvula choke de até $26 \%$, para valores maiores, o sistema se torna instável. As simulações de resposta ao degrau estão mostradas na Figura 4-12. 

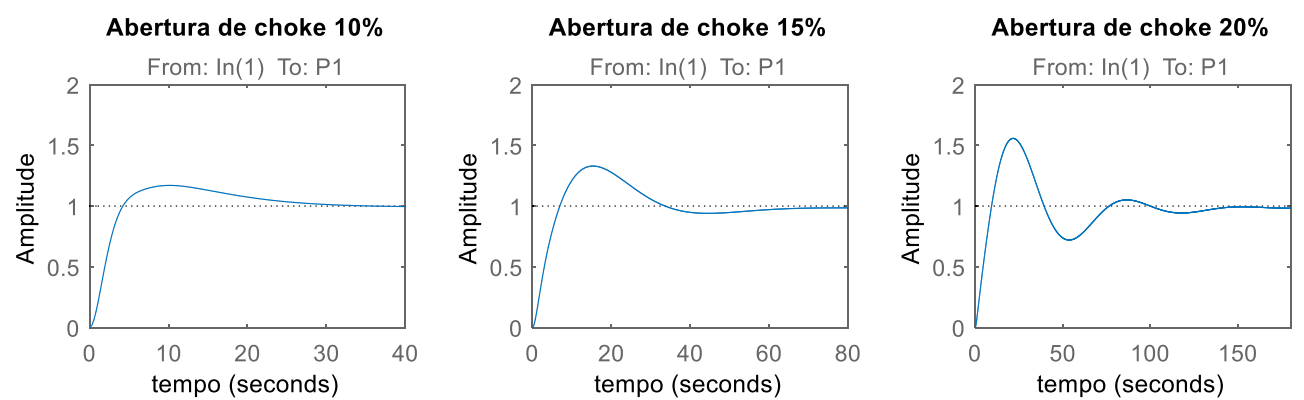

Abertura de choke $25 \%$

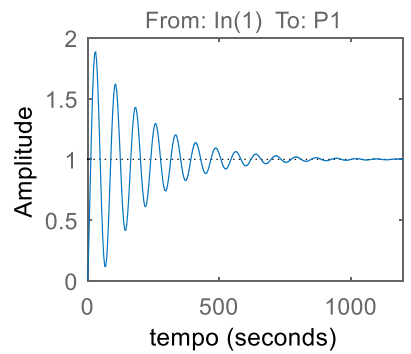

Abertura de choke $30 \%$

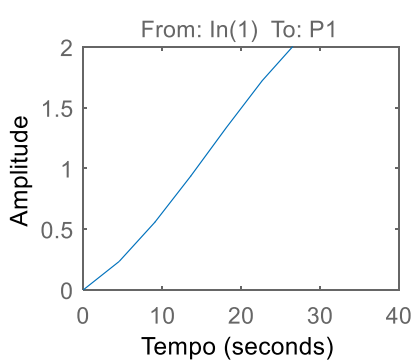

Abertura de choke $35 \%$

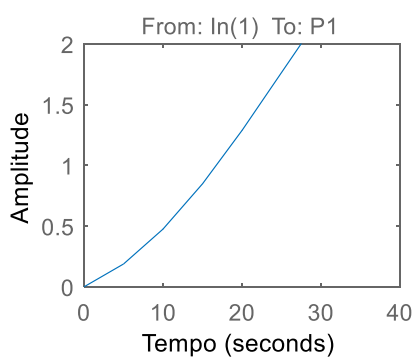

Abertura de choke $45 \%$

Abertura de choke $\mathbf{5 0 \%}$
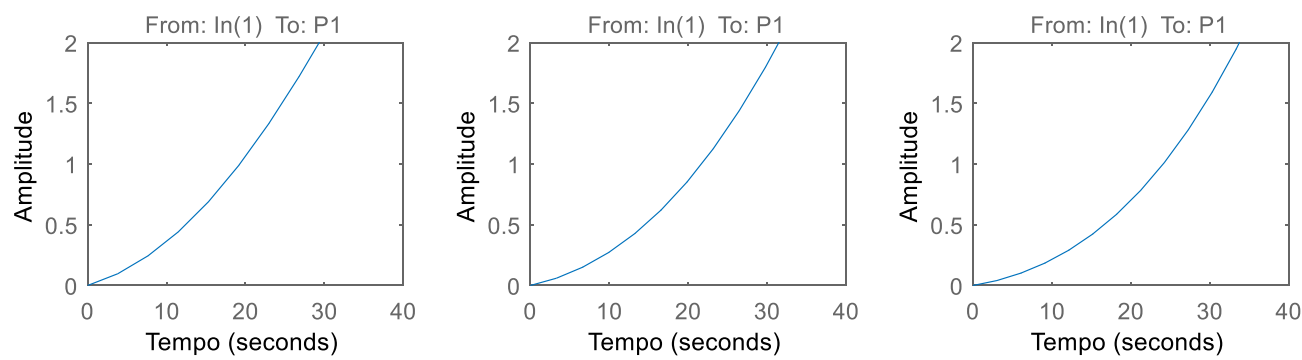

Figura 4-12 - Resposta ao degrau de $P 1$ para aberturas da válvula choke variando de $10 \%$ a $50 \%$ utilizando um controlador PI de ganho estático

Adotando-se o controlador PI adaptativo descrito na seção 4.3.1, obteve-se novamente a resposta em degrau para aberturas da válvula choke entre $10 \%$ e $50 \%$ no MATLAB.

O período de oscilação adotado foi obtido através da simulação do sistema em malha aberta, dado pela Figura 4-9 e é de aproximadamente $T_{o s c}=2500 \mathrm{~s}$.

Nota-se que este controlador consegue estabilizar o sistema até mesmo com grandes aberturas da válvula choke, ou seja, o controlador PI adaptativo apresenta desempenho melhor que o controlador PI de ganho estático, visto que proporciona maior produtividade para o poço através da menor contrapressão imposta ao reservatório pelo fato de operar de maneira estável para maiores aberturas da válvula choke. As simulações de resposta ao degrau para o controlador PI adaptativo estão mostradas na Figura 4-13. 

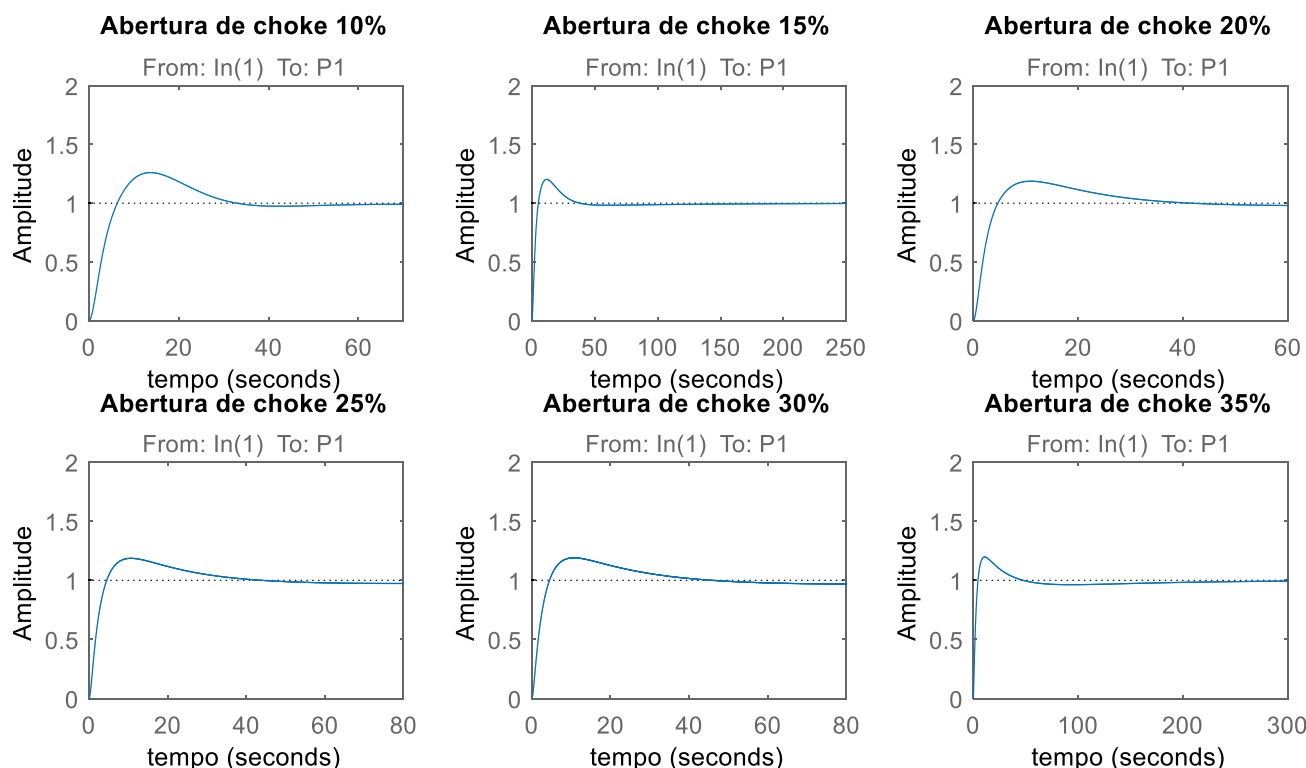

Abertura de choke $30 \%$

Abertura de choke $35 \%$

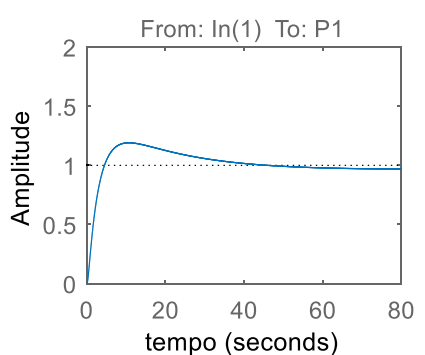

From: $\ln (1)$ To: P1 Abertura de choke $40 \%$

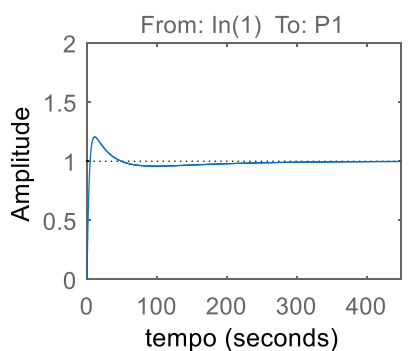
Abertura de choke $\mathbf{4 5 \%}$

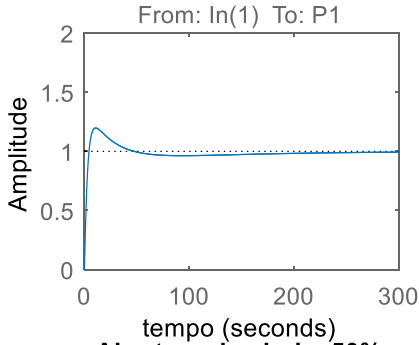

From: $\ln (1)$ To: $\mathrm{P} 1$
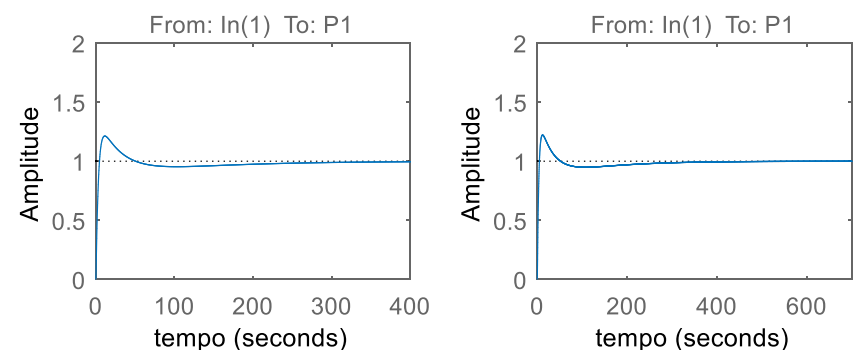

Figura 4-13 - Resposta ao degrau de $P 1$ para aberturas da válvula choke variando de $10 \%$ a $50 \%$ utilizando um controlador PI adaptativo

A Figura 4-14 mostra a variação do ganho proporcional representada pela linha tracejada e do tempo integral representada pela linha cheia, para aberturas da válvula choke variando de $10 \%$ a $50 \%$. 


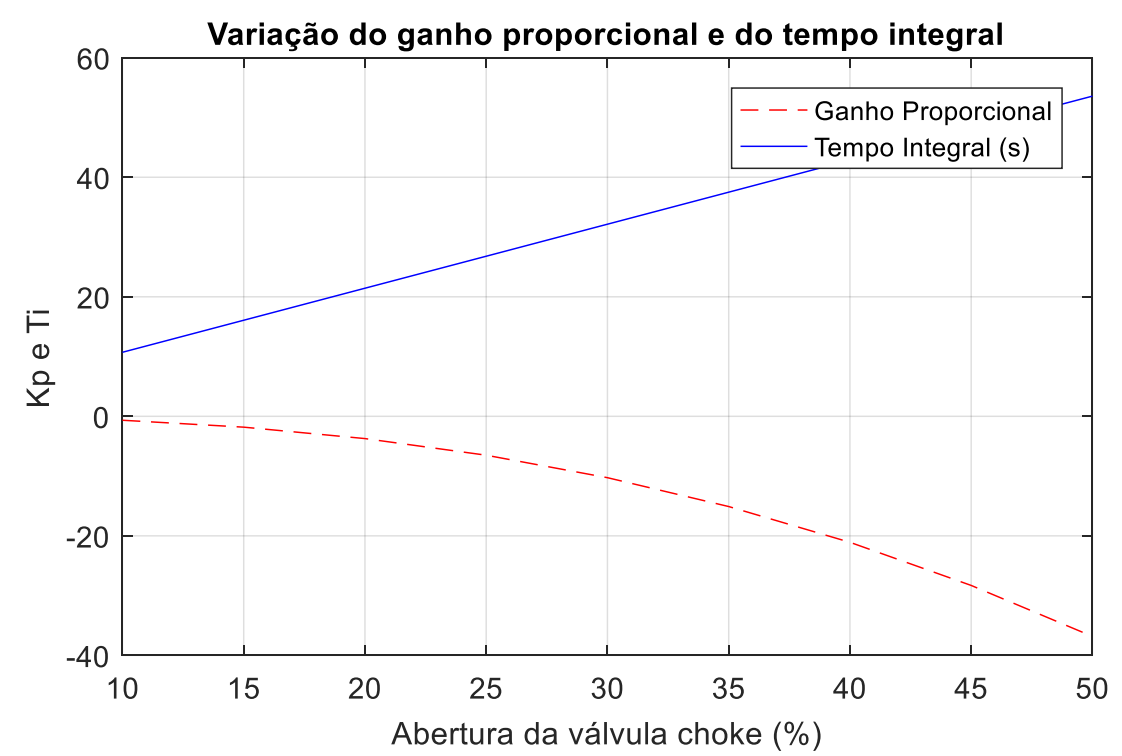

Figura 4-14 - Variação do ganho proporcional e do tempo integral do controlador PI adaptativo para diferentes aberturas da válvula choke

Para a simulação com o OLGA ${ }^{\circledR}$, substituiu-se a planta pelo modelo do OLGA ${ }^{\circledR}$ utilizando os blocos de escrita e leitura de variáveis no servidor OPC disponíveis no Simulink. A configuração do servidor OPC está descrita em detalhes no Apêndice A e a implementação no Simulink do controlador PI adaptativo está mostrada no Apêndice B.

A Figura 4-15 mostra o comportamento da pressão de entrada do pipeline $P 1$ em malha aberta e após a ativação do controlador PI adaptativo. Nota-se que o sistema era oscilatório em malha aberta, para uma abertura fixa da válvula choke em $10 \%$. Após aproximadamente $5 \mathrm{~h}$, o controlador foi ligado e o setpoint da pressão $P 1$ fixado em 68 bar. A válvula choke iniciou a modulação e foi capaz de reduzir drasticamente a oscilação do sistema. Realizou-se a redução do setpoint de pressão em 0,5 bar a cada $5 \mathrm{~h}$, nota-se que o controle adaptativo conseguiu manter as oscilações de pressão em patamares aceitáveis ao mesmo tempo que proporcionou uma pressão média de entrada do pipeline P1 menor que no sistema em malha aberta. A vantagem deste controlador para o $\mathrm{PI}$ convencional é que o mesmo tem a capacidade de operar em ranges maiores da válvula choke, porém este controlador tem a desvantagem de depender da disponibilidade da pressão de entrada do pipeline para operar. 

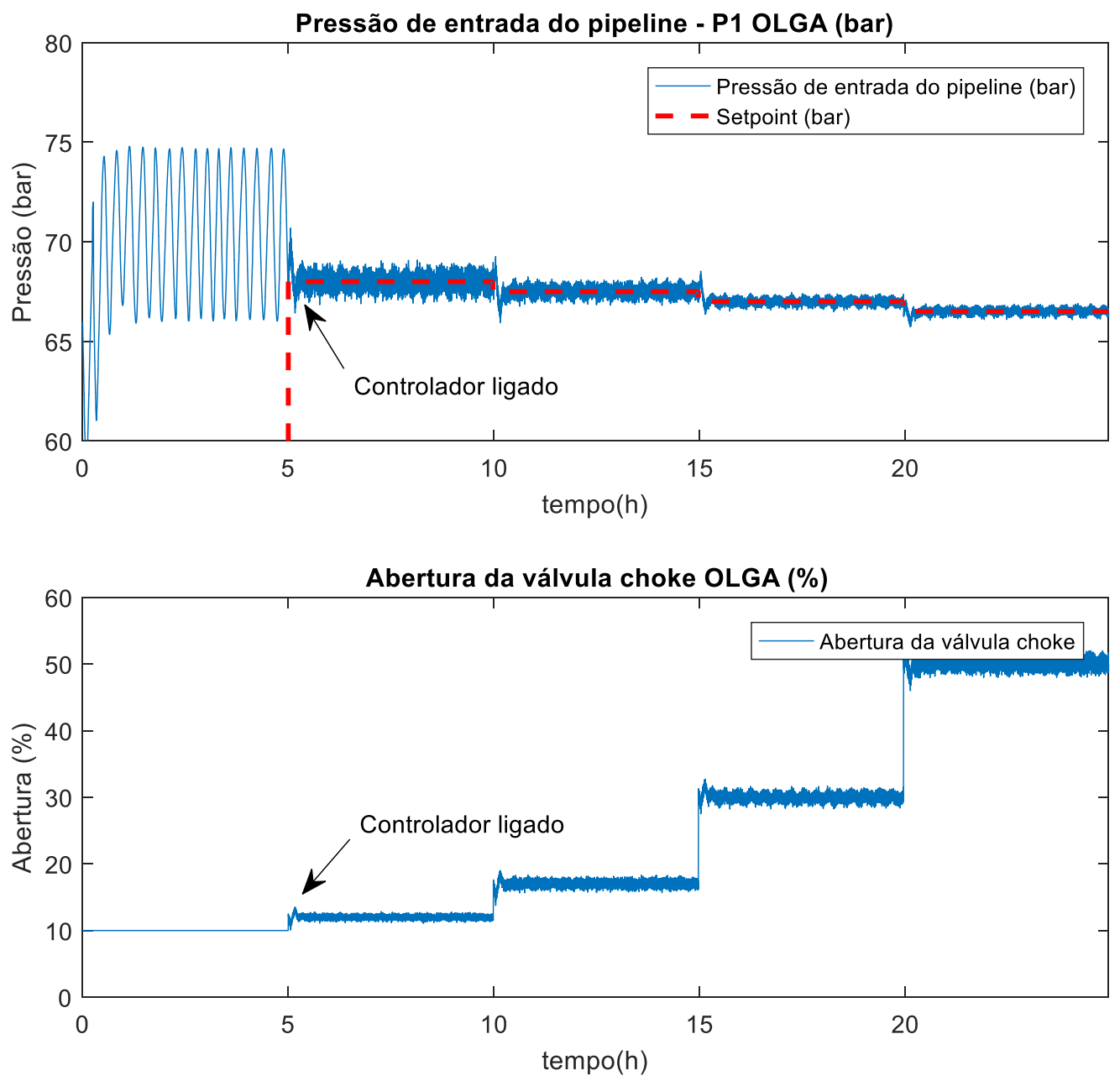

Figura 4-15 - Comportamento da pressão de entrada do pipeline $P 1$ e abertura da válvula choke com a utilização do controlador adaptativo PI para diferentes setpoints de pressão

\subsubsection{Regulador Linear Quadrático com Filtro de Kalman Unscented}

Algumas vezes, os sensores de pressão e temperatura localizados no fundo do mar sofrem falhas, sendo que, muitas vezes a substituição dos mesmos apresentam custos proibitivos. Portanto, é interessante analisar formas de evitar golfadas em sistemas pipeline-riser utilizando apenas a medição de pressão no topside. Porém, a dinâmica desse sistema possui polos próximos a instabilidade (RHP right half plane), (STORKAAS; SKOGESTAD, 2007), além de zeros positivos relativamente próximos aos polos instáveis, como exaustivamente demonstrado na seção 4.1 , de forma prática através dos diagramas de bifurcação. Então, pode-se concluir que a medição real da pressão na entrada do pipeline não é uma tarefa simples, pois há excesso de 
oscilações e ruídos. Uma solução convencional é adotar um observador que utilize a pressão do topside em lugar da pressão do pipeline, para estimar os estados não disponíveis, incluindo a pressão de fundo (JAHANSHAHI, 2013). Em resumo, algumas variáveis são difíceis de serem medidas, portanto é necessário estimar estes estados através das saídas do sistema. Os estados estimados podem ser utilizados com feedback em substituição dos estados reais do sistema (DUTTON; THOMPSON; BARRACLOUGH, 1997).

O Filtro de Kalman proporciona um bom método de estimar o estado de um sistema dinâmico na presença de ruído. Considerando as vantagens expostas na seção 3.4.2, adotou-se neste trabalho o filtro de Kalman Unscented. O desempenho do filtro de Kalman Unscented é melhorado pela implementação de um fator de esquecimento menor que um, com isto, é possível aumentar artificialmente a covariância e o ganho $K$ do observador. Porém, com altos ganhos no observador, o sistema se torna mais sensível ao ruído.

Primeiramente, realizou-se a simulação via MATLAB utilizando o modelo linearizado como planta. A Figura 4-16 mostra o comportamento das pressões de entrada do pipeline $P 1$ e de topo do riser $P 2$. Nota-se que, assim como o controlador PI adaptativo, o regulador linear quadrático com filtro de Kalman Unscented é capaz de estabilizar as oscilações de pressão em patamares aceitáveis ao mesmo tempo que proporciona uma pressão média de entrada do pipeline menor que no sistema em malha aberta. Esta abordagem possui a vantagem de funcionar mesmo em caso de falha do sensor de fundo e na presença de ruídos na medição das variáveis, porém com ranges de aberturas da válvula choke mais restritas que para o caso do controlador PI adaptativo com a medição direta da pressão de fundo. 

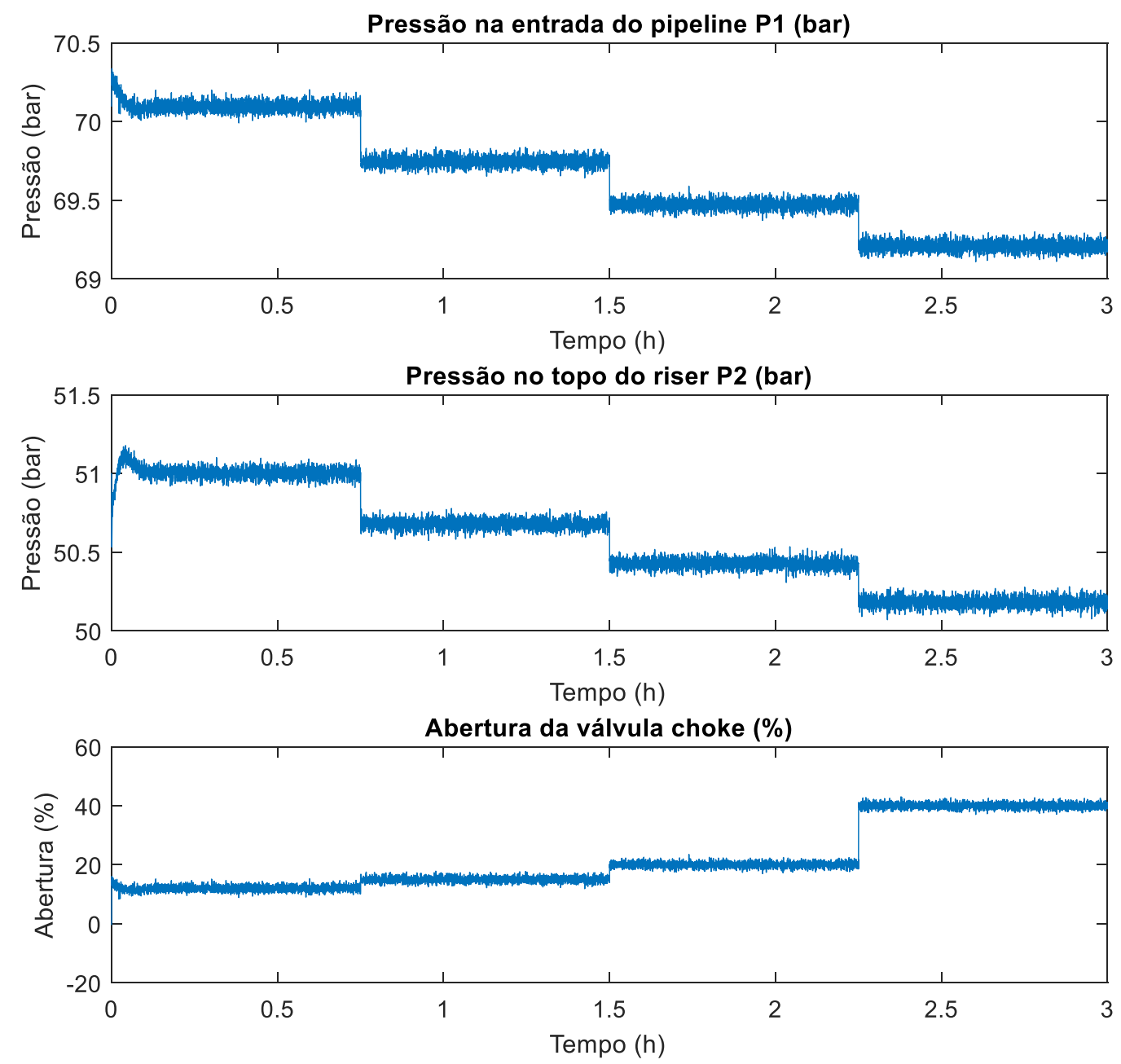

Figura 4-16 - Pressões $P 1$ e $P 2$ com controlador LQR + Filtro de Kalman Unscented para diferentes aberturas da válvula choke

Para a simulação com o software $\mathrm{OLGA}^{\circledR}$, substituiu-se a planta pelo modelo do OLGA $^{\circledR}$ utilizando os blocos de escrita e leitura de variáveis no servidor OPC disponíveis no Simulink. A configuração do servidor OPC está descrita em detalhes no Apêndice A e a implementação no Simulink do regulador linear quadrático com filtro de Kalman Unscented está mostrada no Apêndice C.

A Figura 4-17 mostra o comportamento da pressão de entrada do pipeline $P 1$, pressão de topo do riser $P 2$ para a simulação com o OLGA ${ }^{\circledR}$. Nota-se que, o regulador linear quadrático com filtro de Kalman Unscented só é capaz de estabilizar as oscilações de pressão para aberturas da válvula choke de até aproximadamente $30 \%$, desempenho 
próximo do obtido com o PI convencional, esta diferença se dá principalmente pelo fato de o PI considerar a presença do sensor de entrada do pipeline e a abordagem desta seção considerar apenas a estimativa do sensor, ou seja, apesar do desempenho não tão satisfatório, esta abordagem possui a vantagem de funcionar mesmo em caso de falha do sensor de fundo e na presença de ruídos na medição das variáveis, porém em ranges mais restritos de abertura da válvula choke.
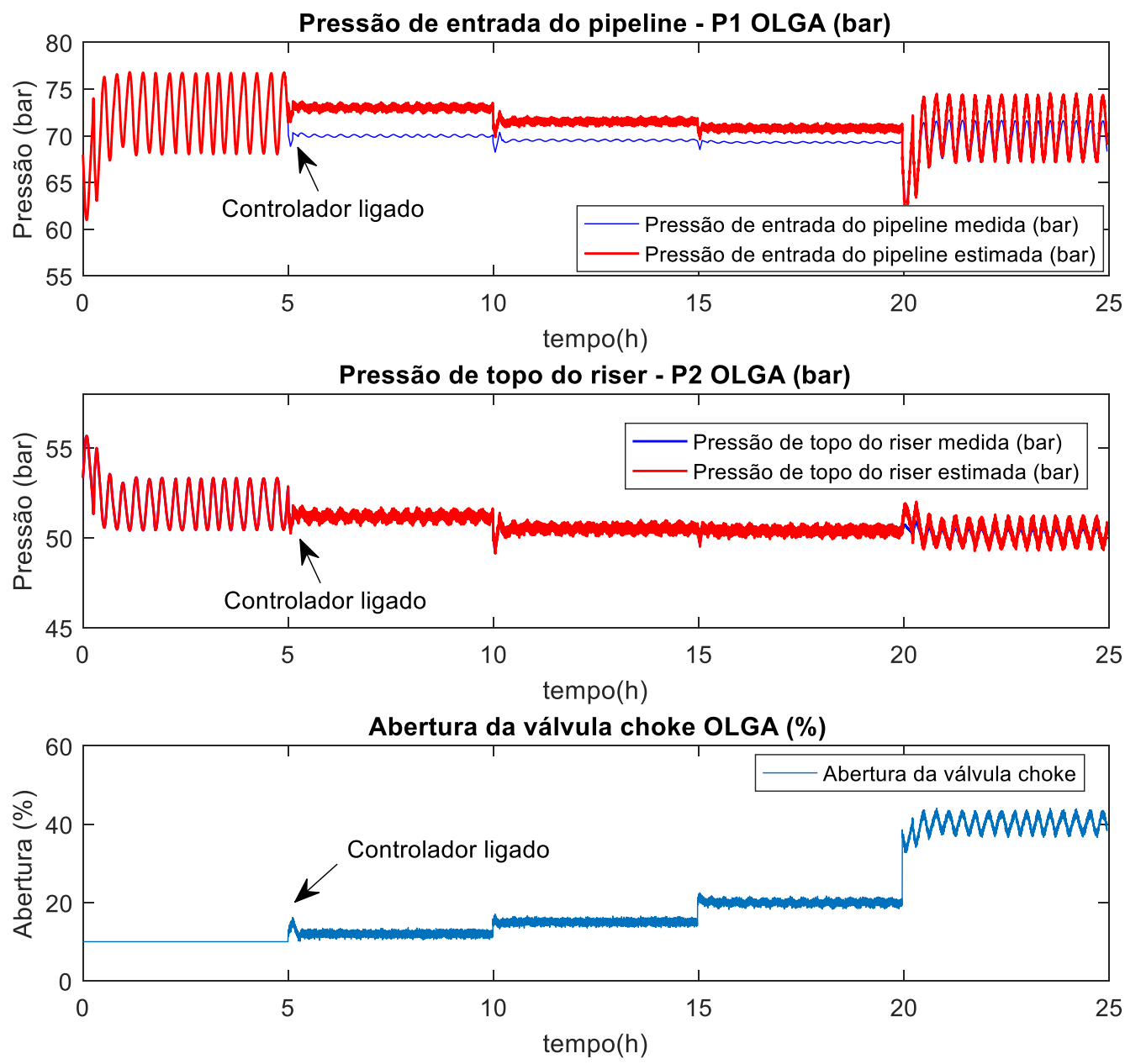

Figura 4-17 - Simulação do OLGA ${ }^{\circledR}$ para as pressões $P 1$ e $P 2$ com o controlador LQR + Filtro de Kalman Unscented 


\section{CONCLUSÕES}

O estudo da estabilidade em escoamentos utilizando a válvula choke em plataformas offshore de produção de petróleo é um problema bem estabelecido e definido, incluindo a modelagem, a análise e a representação matemática, como já descrito no presente trabalho.

Entretanto, a solução de controle ou mitigação dessa instabilidade ainda é um problema em aberto. Toda a literatura publicada ou os laboratórios associados ${ }^{2}$ apresentam soluções para este problema visando aplicações práticas, com métodos teóricos e técnicas de controle relativamente simples, visto que o problema por si só já é extremamente complexo. $O$ presente trabalho não poderia ser diferente, apresentando soluções de ponto de vista prático com teorias de controle já consolidadas.

Nas seções 4.1 e 4.2, concluiu-se que a dinâmica do modelo simplificado implementado no MATLAB representa de forma satisfatória os resultados obtidos no simulador OLGA ${ }^{\circledR}$, possibilitando a adoção do modelo do MATLAB para o projeto dos algoritmos de controle desenvolvidos neste trabalho. $A$ implementação dos algoritmos de controle foi realizada no MATLAB/Simulink e sua aplicação no modelo do OLGA ${ }^{\circledR}$ foi realizada via servidor $\mathrm{OPC}$, que possibilita a interação na válvula choke em tempo real.

A avaliação da eficácia dos métodos de supressão das golfadas, foi realizada através de duas abordagens distintas. Inicialmente, utilizou-se um controlador PI adaptativo com capacidade de operação em diferentes regiões de aberturas da válvula choke. Por fim, utilizou-se um Regulador Linear Quadrático associado a um observador de estados com filtro de Kalman Unscented com o objetivo de verificar como o sistema

\footnotetext{
2 Ver por exemplo, grupo da NTNU liderado por Skogestad http:/www.nt.ntnu.no/users/skoge/publications/2015/oliveira-ifacoffshore-2015/patent/NTNU\%20Anti-slug\%20control_patent_product\%20sheet\%20(1).pdf; Oil and Gas Engineering Laboratory de Univ. Cranfield https://www.cranfield.ac.uk/case-studies/research-case-studies/slug-control; Statoil de Noruega http://www.statoil.com/en/technologyinnovation/fielddevelopment/flowassurance/slugcontrol/pages/default.aspx, CENPES de Petrobras https://pdfs.semanticscholar.org/09c2/93db97387cfdf832a511df192e4aabb6989c.pdf e http://www.swge.inf.br/CBA2014/anais/PDF/1569996275.pdf, e muito outros centros.
} 
se comportaria em caso de necessidade de um observador para compensar uma possível falha do sensor submarino de pressão.

Conforme mostrado na seção 4.3.1, é possível reduzir as oscilações na pressão de entrada do pipeline $P 1$ após a ativação do controlador PI adaptativo. Nota-se que o sistema era oscilatório em malha aberta, para uma abertura fixa da válvula choke em $10 \%$ mas após a ativação do controlador, a válvula choke iniciou a modulação e conseguiu reduzir as oscilações do sistema. Esta estabilização é notada mesmo reduzindo-se o setpoint de pressão de entrada do pipeline $P 1$, ou seja, foi possível manter as oscilações de pressão em patamares aceitáveis ao mesmo tempo que se obteve uma pressão média menor de entrada do pipeline quando comparada ao sistema em malha aberta.

A seção 4.3.2, mostra o desempenho do regulador linear quadrático combinado com o filtro de Kalman Unscented. Assim como o controlador PI adaptativo, o regulador linear quadrático com filtro de Kalman Unscented foi capaz de estabilizar as oscilações porém, o mesmo só é capaz de obter resultados satisfatórios para aberturas da válvula choke de até aproximadamente $30 \%$, desempenho próximo do obtido com o PI convencional, esta diferença se dá principalmente pelo fato de o PI considerar a presença do sensor de entrada do pipeline e regulador linear quadrático considerar apenas a estimativa do sensor, ou seja, apesar do desempenho menor, esta abordagem possui a vantagem de funcionar mesmo em caso de falha do sensor de fundo e na presença de ruídos na medição das variáveis.

A faixa de operação da válvula choke, tanto para o PI quanto para o LQR, não é um problema em si, uma vez que se pode utilizar um sistema supervisório para a decisão de controle por faixas (model based switched control) resolvendo essa "rangeabilidade" do controlador. Entretanto, do ponto de vista de metodologia, não acrescentaria relevância ao presente estudo. Revisitar as técnicas de controle adaptativo tanto para o PI quanto para o LQR, aliado ao estado da arte dos Filtros de Kalman, atende aos propósitos do presente trabalho.

Em trabalhos futuros, pode-se avaliar a utilização de novas teorias de controle como o controle distribuído de equações diferenciais parciais (PDE based control) ou 
controle estocástico de sistemas inerentemente estocásticas (ou seja, sem uma determinação precisa da variável de saída sendo, portanto, não baseado em realimentação do erro, um exemplo é o controle de sistemas quânticos ${ }^{3}$ ). Entretanto essas incursões teóricas estão além do escopo do presente trabalho.

${ }^{3}$ Performance Measure for Optimal Quantum Control https://arxiv.org/pd//1504.03926.pdf 


\section{REFERÊNCIAS}

ASTOLFI, A.; KARAGIANNIS, D.; ORTEGA, R. Nonlinear and Adaptive Control with Applications. Springer, 2010.

ÅSTRÖM, K. J.; WITTENMARK, B. On self-tuning regulators. Automatica. $p$. 185-199. 1973.

ÅSTRÖM, K. J.; WITTENMARK, B. Adaptive Control. Massachusetts, 1989.

ÅSTRÖM, K. J.; WITTENMARK, B. A. Survey of Adaptive Control applications. Proceedings of the 34th IEEE Conference on Decision and Control. p. 649654. 1995.

AUGER, F.; HILAIRET, M.; GUERRERO, J. M.; MONMASSON, E.; ORLOWSKAKOWALSKA, T.; KATSURA, S. Industrial applications of the Kalman filter A review. IEEE Transactions on Industrial Electronics. p. 60(12), 5458-5471. 2013.

BENDIA, R. M. Avaliação econômica de estratégias de controle de golfadas no processo de separação de plataformas marítimas. Dissertação de Mestrado. UFRJ. Rio de Janeiro. 2013.

BENDIKSEN, K. H.; MALNES, D.; MOE, R.; NULAND, S. The Dynamic Two-Fluid Model OLGA Theory and Application. SPE Production Engineering. p. 171180. 1991.

BILTOFT, J.; HANSEN, L.; PEDERSEN, S.; Z. YANG, Z. Recreating riser slugging flow based on an economic lab-sized setup. IFAC International Workshop on Periodic Control 5th. p. 47-52. 2013.

BURKE, N. E.; KASHOU, S. F. Slug sizing/slug volume prediction, state of the art review and simulation. Offshore Technology Conference. 1995.

BURNS, R. S. Advanced Control Engineering. 1․ ed. Londres Butterworth, 2001.

CAMPOS, M.; TAKAHASHI, T. R.; ASHIKAWA, F. H.; N., S. S.; MEIEN, O. F. V.; STENDER, A. Advanced anti-slug control for offshore production plants. 2nd IFAC Workshop on Automatic Control in Offshore Oil and Gas Production. Florianopolis. p. 83-88. 2015.

COURBOT, A. Prevention of Severe Slugging in the Dunbar 16' Multiphase Pipeline. Offshore Technology Conference. Houston. 1996.

CRUZ, S. M. Implementação de um Filtro de Kalman Estendido em Arquiteturas Reconfiguráveis Aplicado ao Problema de Localização em Robótica Móvel. Dissertação de Mestrado. UnB. Brasília. 2013.

DUTTON, K.; THOMPSON, S.; BARRACLOUGH, B. The Art of Control Engineering. 1‥ ed. 1997.

FARD, M. P.; GODHAVN, J. M.; SAGATUN, S. I. Modeling of Severe Slug and Slug Control with OLGA. SPE International Symposium and Exhibition on Formation Damage Control. Lafayette. 2006. 
GODHAVN, J. M.; FARD, M. P.; FUCHS, P. H. New slug control strategies, tuning rules and experimental results. Journal of Process Control 15. p. 547-557. 2004.

GREWAL, M. S.; ANDREWS, A. P. Kalman filtering theory and practice using Matlab. Wiley, 2008.

HAVRE, K.; DALSMO, M. Active Feedback Control as a Solution to Severe Slugging. SPE Annual Technical Conference and Exhibition. 2002.

HAVRE, K.; STORNES, K. O.; STRAY, H. Taming slug flow in pipelines. ABB Review. 2000.

JAHANSHAHI, E. Control Solutions for Multiphase Flow Linear and nonlinear approaches to anti-slug control. Tese de Doutorado. NTNU. Trondheim. 2013.

JAHANSHAHI, E.; OLIVEIRA, V.; GRIMHOLT, C.; SKOGESTAD, S. A comparison between Internal Model Control, optimal PIDF and robust controllers for unstable flow in risers. Elsevier IFAC Publications. p. 57525759. 2014.

JAHANSHAHI, E.; SKOGESTAD, S. Simplified Dynamical Models for Control of Severe Slugging in Multiphase Risers. 18th IFAC World Congress. Milão,. p. 1634-1639. 2011.

JAHANSHAHI, E.; SKOGESTAD, S. Comparison between nonlinear modelbased controllers and gain-scheduling Internal Model Control based on identified model. Proceedings of the IEEE Conference on Decision \& Control. p. 853-860. 2013.

JAHANSHAHI, E.; SKOGESTAD, S. Anti-slug control solutions based on identified model. J. Process Control. 2015.

JANSEN, F. E.; SHOHAM, O.; TAITEL, Y. The elimination of severe sluggingexperiments and modeling. Int. J. Multiphase Flow Vol. 22. p. 1055-1072. 1996.

JULIER, S. J.; UHLMANN, J. K. A general method for approximation nonlinear transformations of probability distributions. Technical report, Robotics Research Group, Department of Engineering Science,. 1996.

JULIER, S. J.; UHLMANN, J. K. A new extension of the kalman filter to nonlinear systems. In: AeroSense: The 11th Internacional Symposium on Aerosparce/Defense Sensing, Simulation and Controls. 1997.

JULIER, S. J.; UHLMANN, J. K. Unscented filtering and nonlinear estimation. Proceedings of the IEEE. p. 92(3), 401-422. 2004.

JUNIOR, H. B. Métodos de Estimação Recursiva Baseados no Filtro de Kalman Aplicados a Sistemas Não-Lineares. Dissertação de Mestrado. UFMG. Belo Horizonte. 2003.

KAASA, G. O.; ALSTAD, V.; ZHOU, J.; AAMO, O. M. Nonlinear model-based control of unstable wells. Modeling, Identification and Control. p. 69-79. 2007.

KANIESKI, J. M. Modelagem e Controle LQR Aplicado a um Condicionador de Energia. Dissertação de Mestrado. UTFPR. Pato Branco. 2010. 
LAGES, W. F. Controle Adaptativo de Sistemas Estocásticos. UFRGS. Porto Alegre. 2007.

LAWRENCE, C.; XU, Z. G.; ANDERSSON, P.; EIDING, H.; HOVDEN, L. .; HENRIKSSON, J.; HU, B. Validation of a Physically-Based Model for Slug Initiation and Evolution in Hydrodynamic Slug Flow. In 17th International Conference on Multiphase Production Technology. BHR Group. 2015.

MARRELS, I. M. Y.; ANDERSON, B. D. O.; BITMEAD, R. R.; BODSON, M.; SASTRY, S. S. Revisition the MIT rule for adaptive control. In Proceedings of the 2nd IFAC Workshop on Adaptive Systems in Control and Signal Processing. 1986.

MEGLIO, F. D. Dynamics and control of slugging in oil production. Dissertação de Mestrado. MINES ParisTech. Paris. 2011.

MEGLIO, F. D.; KAASA, G. O.; PETIT, N. A first principle model for multiphase slugging flow in vertical risers. Joint 48th IEEE Conference on Decision and Control and 28th Chinese Control Conference. 2009.

MERWE, R. V.; WAN, E. A. Kalman Filtering and Neural Networks cap 7. Ontario. John Wiley \& Sons, 2001.

NARENDRA, K. S.; ANNASWAMY, A. M. Stable Adaptive Systems. Dover Publications, 1989.

NARENDRA, K. S.; LIN, Y. H.; VALAVANI, L. S. Stable adaptive controller design, part II Proof of stability. IEEE Transactions on Automatic Control. p. 440-448. 1980.

OGAZI, A. I.; CAO, Y.; YEUNG, H.; LAO, L. Slug control with large valve opening to maximizing oil production. Offshore Europe Oil \& Gas Conference \& Exhibition. 2010.

OLIVEIRA, C. A. M. Projeto de Observadores de Estado utilizando Algoritmos Genéticos. Dissertação de Mestrado. UFERSA. Mossoró. 2014.

OLIVEIRA, V.; JÄSCHKE, J.; SKOGESTAD, S. An autonomous approach for driving systems towards their limit an intelligent adaptive anti-slug control system for production maximization. 2nd IFAC Workshop on Automatic Control in Offshore Oil and Gas Production. 2015.

PEDERSEN, S.; DURDEVIC, P.; YANG, Z. Learning control for riser-slug elimination and production-rate optimization for an offshore oil and gas production process. The 19th World Congress of the International Federation of Automatic Control. p. 8522-8527. 2014.

PEDERSEN, S.; DURDEVIC, P.; YANG, Z. Challenges in slug modeling and control for offshore oil and gas productions: A review study. International Journal of Multiphase Flow Volume 88. p. 270-284 Jan. 2017.

PICKERING, P. F.; HEWITT, G. F.; WATSON, M. J.; HALE, C. P. The prediction of flows in production risers - truth \& myth? IIR Conference. 2001.

SALES, F. C. S. Análise da Acurácia do Simulador de Escoamento Bifásico OLGA em Transientes de Gasodutos. Dissertação de Mestrado. UFRJ. Rio de Janeiro. 2013. 
SASTRY, S.; BODSON, M. Adaptive Control Stability, Convergence and Robustness. Dover Publications, 1989.

SCHLUMBERGER. OLGA User Manual. Dynamic Multiphase Flow Simulator. 2014.

SCHMIDT, Z.; BRILL, J.; BEGGS, D. Riser-base gas injection into the se forties line. SPE Journal. p. 407-414. 1980.

SILVA, C. M.; DESSEN, F.; NYDAL, O. J. Dynamic multiphase flow models for control. 7th North American Conference on Multiphase Technology. p. 221236. 2010.

SILVA, M. J. S. Controle Adaptativo Aplicado a um Sistema de Bombeamento de Água Visando a Redução de Perdas de Energia Elétrica e Água. Tese de Doutorado. UFPB. João Pessoa. 2014.

SIVERTSEN, H.; E. STORKAAS, E.; SKOGESTAD, S. Small-scale Experiments on Stabilizing Riser Slug Flow. Chemical Engineering Research and Design. p. 213-228. 2010.

SIVERTSEN, H.; SKOGESTAD, S. Cascade Control Experiments of Riser Slug Flow using Topside Measurements. 2005.

SKOGESTAD, S. Chemical And Energy Process Engineering. CRC Press, Taylor \& Francis Group, Boca Raton, FL, 2009.

STORKAAS, E.; SKOGESTAD, S. A simple dynamic model for control design and analysis of severe slugging. Multiphase'03. 2003.

STORKAAS, E.; SKOGESTAD, S. Controllability analysis of two-phase pipeline-riser systems at riser slugging conditions. Control Engineering Practice 15. p. 567-581. 2007.

ST-PIERRE, M.; GINGRAS, D. Comparison between the Unscented Kalman filter and the extended Kalman filter for the position estimation module of an integrated navigation information system. Intelligent Vehicles Symposium, 2004 IEEE. p. 831-835. 2004.

SUN, L.; GAN, J. Researching of two-wheeled self-balancing robot base on LQR combined with PID. 2nd International Workshop on Intelligent Systems and Applications. p. 1-5. 2010.

SYRE, T. V. Anti-Slug Control with Non-Linear State Estimation. Dissertação de Mestrado. NTNU. Trondheim. 2012.

TAITEL, Y. Stability of severe slugging. Int. J. Multiphase Flow Vol. 12. p. 203217. 1986.

THRUN, S.; BURGARD, W.; FOX, D. Probabilistic Robotics. The MIT Press, 2005.

UYGUN, K.; MATTHEW, H. W. T.; HUANG, Y. FBA-LQR An optimal control approach to flux balance analysis. Industrial e Engineering Chemistry Research. p. 8554-8564. 2006.

WAN, E. A.; MERWE, R. V.; NELSON, A. T. Dual Estimation and the Unscented Transformation, volume Neural Information Processing Systems. p. 666672. 2000. 
YOCUM, B. Offshore riser slug flow avoidance Mathematical models for design and optimization. SPE European Meeting. Londres,. p. SPE 4312. 1973.

ZAKARIAN, E.. Analysis of two-phase flow instabilities in pipe-riser systems. In: Proceedings of the ASME pressure vessels and piping conference, Seattle. 2000.

ZHOU, J.; KAASA, G. O.; AAMO, O. M. Nonlinear adaptive observer control for a riser slugging system in unstable wells. American Control Conference Westin Seattle Hotel. Seattle. 2008. 


\section{APÊNDICE A}

\section{Integração MATLAB com o OLGA ${ }^{\circledR}$}

Utilizou-se o servidor OPC para viabilizar a comunicação entre os softwares OLGA ${ }^{\circledR} \mathrm{e}$ MATLAB. Abaixo, seguem descritos o passo a passo para configurar o servidor. Após a modelagem do sistema no software $O L G A^{\circledR}$, realiza-se a configuração servidor OPC através do item "SERVEROPTIONS". Nesta tela, é necessário preencher as opções básicas do servidor OPC conforme mostrado na Figura A-1.

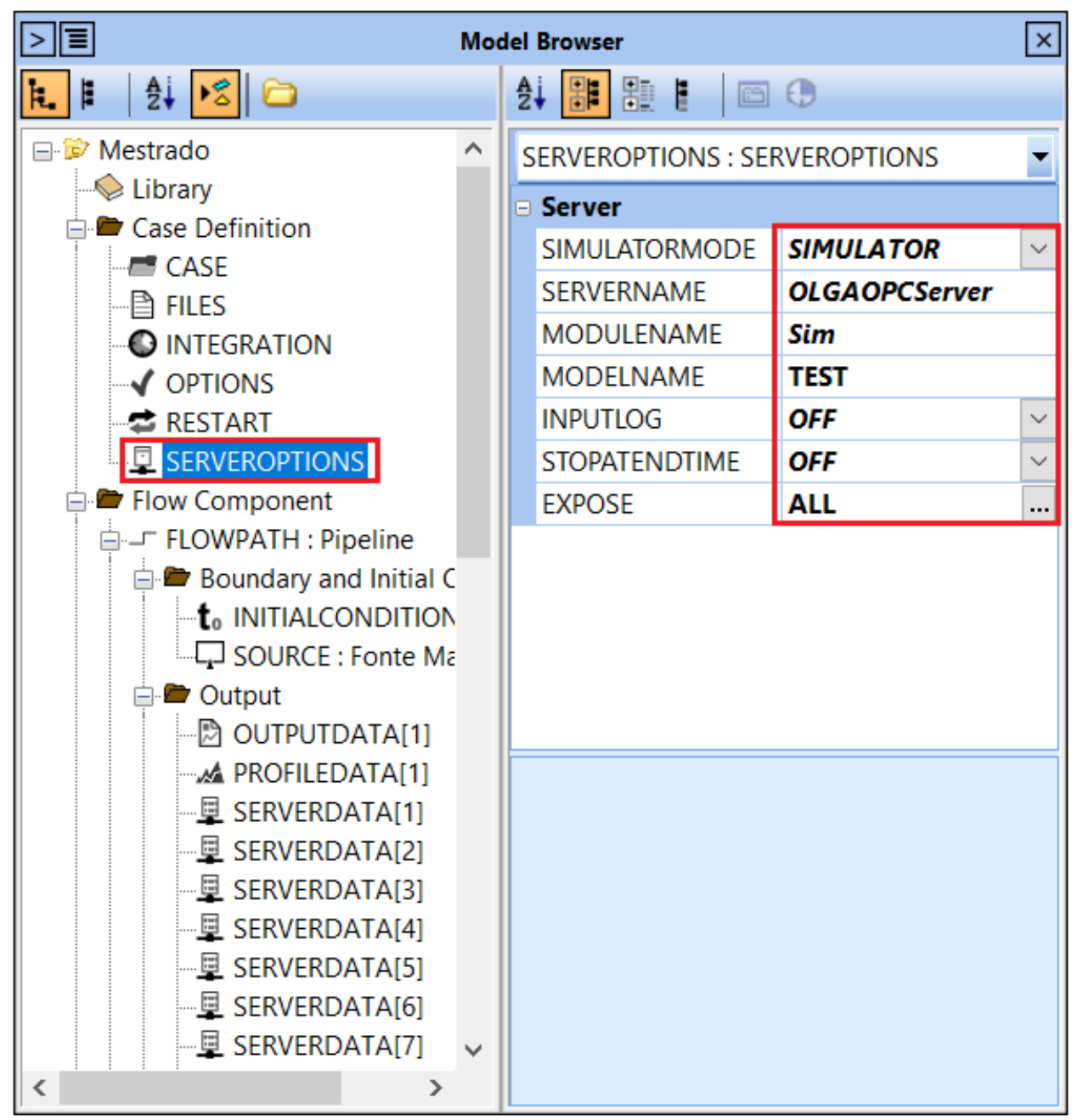

Figura A-1 - Tela de configurações básicas do servidor OPC no OLGA ${ }^{\circledR}$

O próximo passo consiste em expor no servidor OPC, as variáveis desejadas para leitura e escrita, isto é realizado incluindo itens "SERVERDATA" nas saídas do 
modelo. Cada variável deve ser configurada individualmente conforme mostrado na Figura A-2.

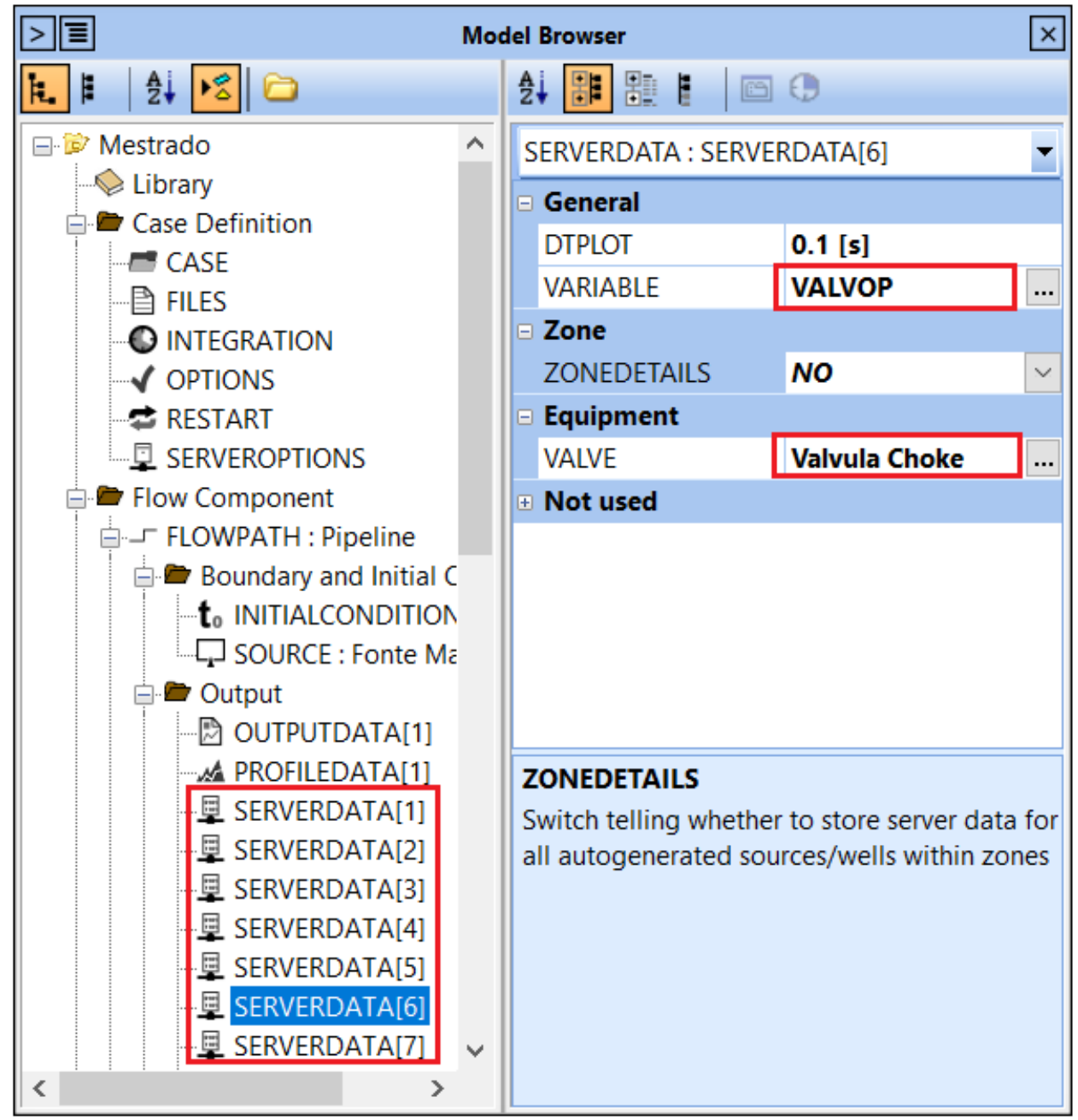

Figura A-2 - Tela de configuração das variáveis a serem expostas no servidor OPC

O passo seguinte consiste em iniciar a simulação do modelo em modo "batch" conforme mostrado na Figura A-3.

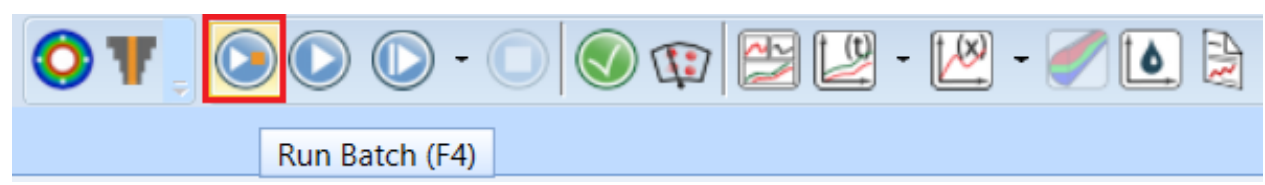

Figura A-3 - Barra de tarefas para início da simulação em "batch"

Ao iniciar a simulação em modo "batch", a tela de status da simulação é aberta, mostrando os dados de registro do servidor OPC e o tempo decorrido de simulação, conforme mostrado na Figura A-4. 


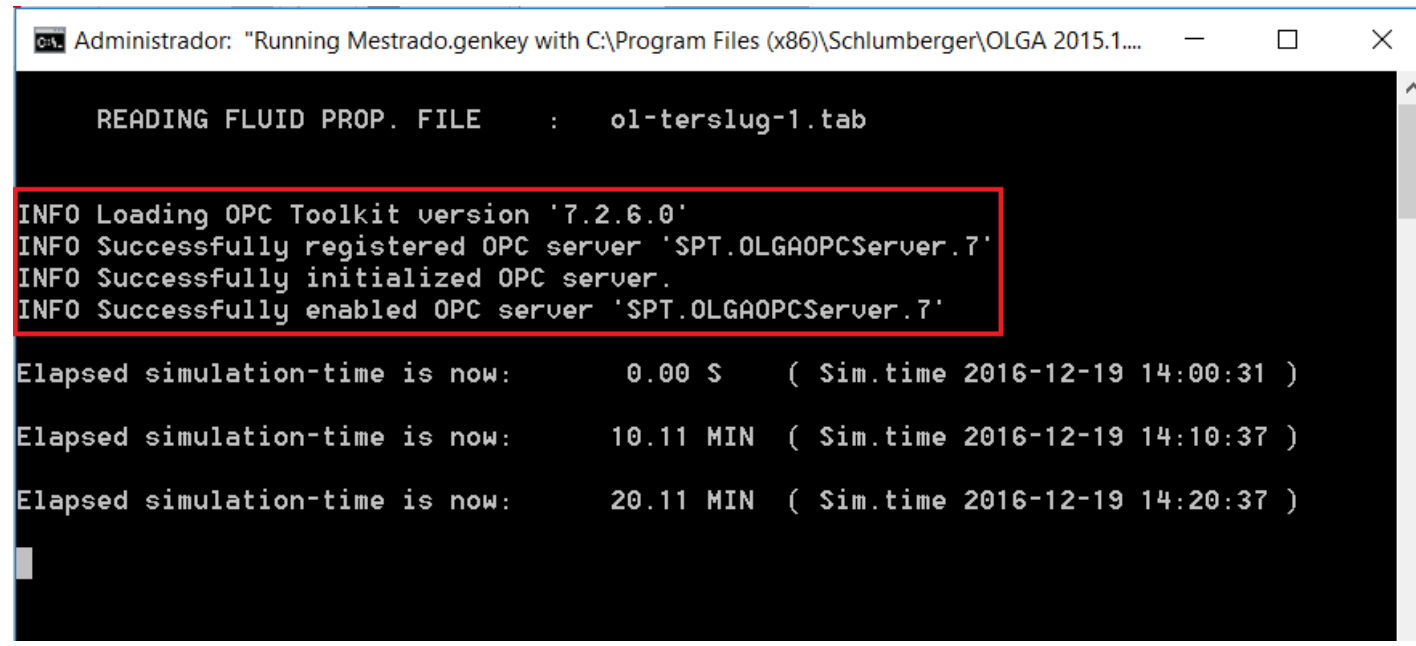

Figura A-4 - Tela de status da simulação

Nesta etapa, o servidor OPC já está registrado e o OLGA ${ }^{\circledR}$ já está enviando e recebendo dados do servidor. O próximo passo consiste em realizar a configuração do MATLAB para que a comunicação seja estabelecida por completo. A configuração do servidor do MATLAB pode ser realizada via Simulink, através do bloco "OPC Configuration". Ao abrir as configurações do bloco em questão, é possível conectar ao servidor recém registrado, estabelecendo a comunicação conforme mostrado na Figura A-5.

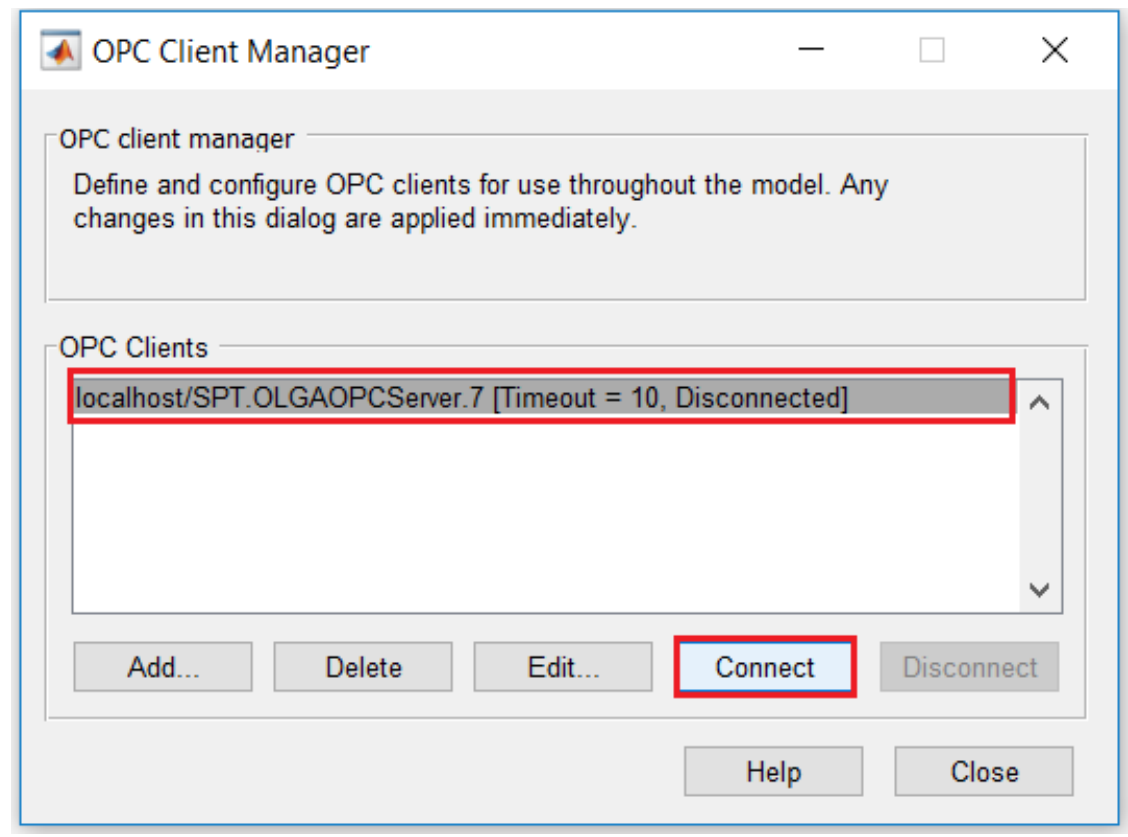

Figura A-5 - Tela de configuração do bloco do Simulink "OPC Configuration" 
Por fim, basta adicionar no Simulink, os blocos de escrita e leitura do servidor OPC e escolher as variáveis desejadas. Estes blocos estão disponíveis na biblioteca do OPC Toolbox do Simulink conforme mostrado na Figura A-6.

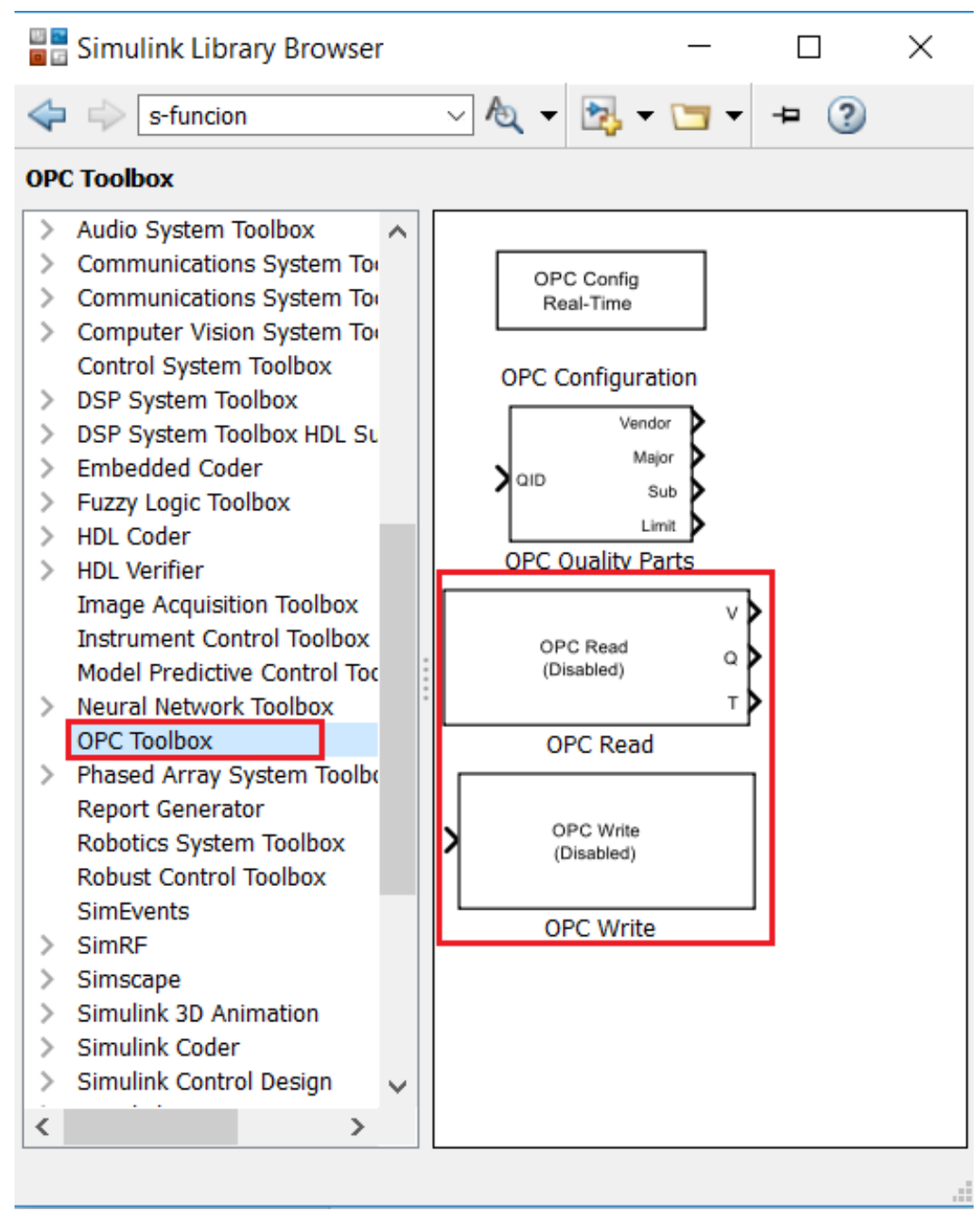

Figura A-6 - Blocos do Simulink para leitura e escrita de variáveis do servidor OPC 


\section{APÊNDICE B}

\section{Implementação do controlador PI adaptativo no Simulink}

Primeiramente, implementou-se o controlador adaptativo no Simulink utilizando como planta, o modelo linearizado para a abertura da válvula choke em $10 \%$, obtendo-se assim, a função de transferência com a abertura $z$ da válvula choke como entrada e a pressão de entrada do pipeline $P 1$ como saída.

A implementação do algoritmo de adaptação se deu através da criação de uma função no MATLAB que contém as equações mostradas na seção 4.3.1. Esta função utiliza como entrada a abertura $z$ da válvula choke e como saída os ganhos proporcional $K c$ e integral $K i$, que por sua vez são entradas do controlador PI adaptativo.

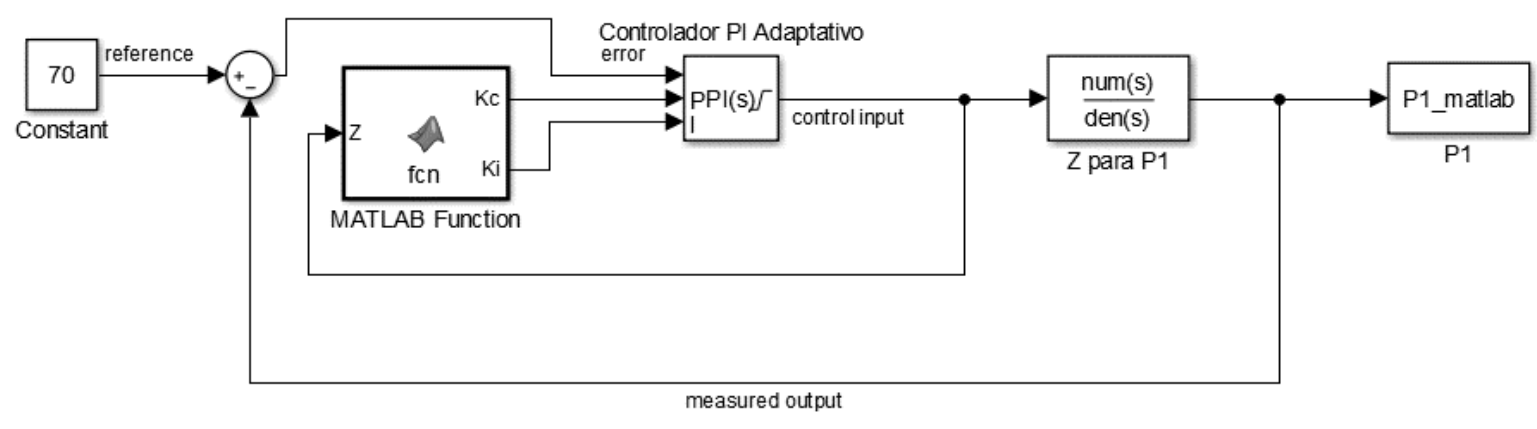

Figura B-1 - Diagrama Simulink do controlador PI adaptativo utilizando o modelo linearizado do MATLAB

Após a implementação utilizando a função de transferência linearizada do MATLAB, realizou-se a implementação do sistema utilizando a planta do OLGA ${ }^{\circledR}$ como sistema a ser controlado. Para isto, utilizou-se blocos do Simulink para escrita e leitura de variáveis de forma a possibilitar a troca de informação entre os dois softwares. 


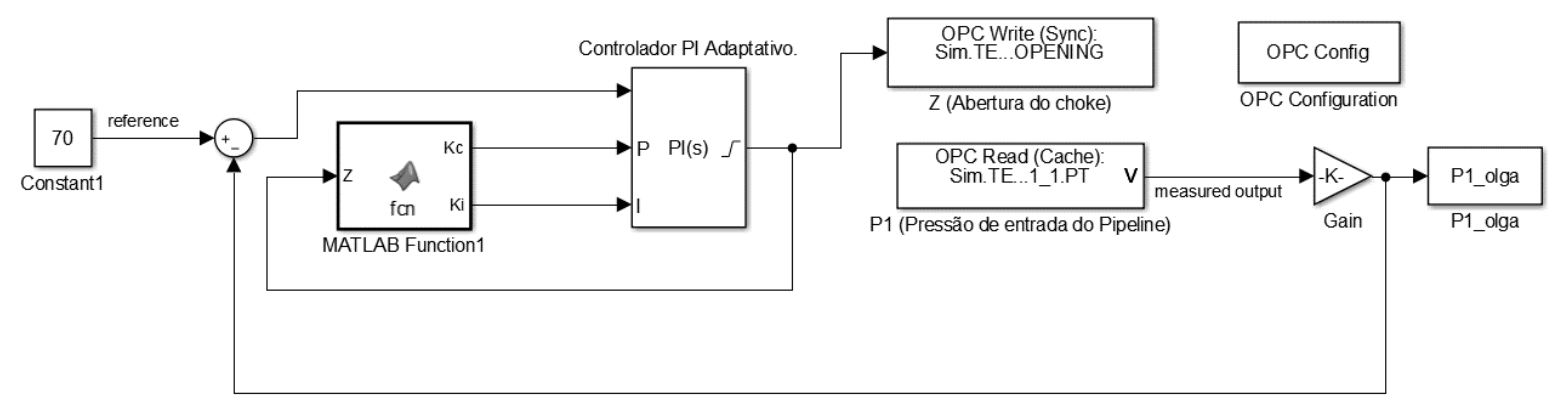

Figura B-2 - Diagrama Simulink do controlador PI adaptativo utilizando o modelo do OLGA ${ }^{\circledR}$

\section{Código do MATLAB para o algoritmo de adaptação}

function $[\mathrm{Kc}, \mathrm{Ki}]=\mathrm{fcn}(\mathrm{Z})$

wG_in0 $=0.36$; \%vazão de entrada gás

$w \mathrm{~L} \_$in $0=8.64 ; \%$ vazão de entrada líquido

beta $=0.061$;

tosc $=2500 ; \%$ segundos

z critico $=0.07$;

$\mathrm{T} 2=2.903 \mathrm{e}+02$

rho_L= 8.322e+02;

$\mathrm{R}=8314$;

M_G= 23;

$w=9$;

$\mathrm{Cv}=1.16 \mathrm{e}-2$;

$\mathrm{P} 2 \mathrm{t}=57.8 \mathrm{e} 5$;

rho_G2=P2_t*M_G/(R*T2);

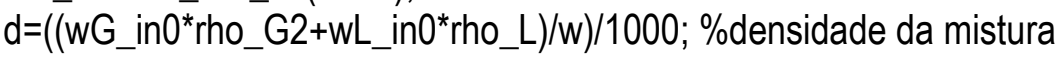
$a=(1 / d)^{*}(w / C v)$;

$k z=-2^{*} a /\left(Z^{\wedge} 3\right)$;

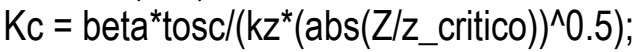

$\mathrm{Ti}=3^{*} \operatorname{tosc}^{*}\left(\mathrm{Z} / \mathrm{z} \_\right.$critico);

$\mathrm{Ki}=1 / \mathrm{Ti}$

end 


\section{APÊNDICE C}

\section{Implementação do regulador linear quadrático (LQR) com Filtro de Kalman Unscented}

Primeiramente, implementou-se o regulador linear quadrático (LQR) com Filtro de Kalman Unscented no Simulink utilizando como planta, o modelo linearizado para a abertura da válvula choke em 10\%, obtendo-se assim, a função de transferência com a abertura $z$ da válvula choke como entrada e a pressão de entrada do pipeline $P I$ como saída conforme mostrado na Figura C-1.

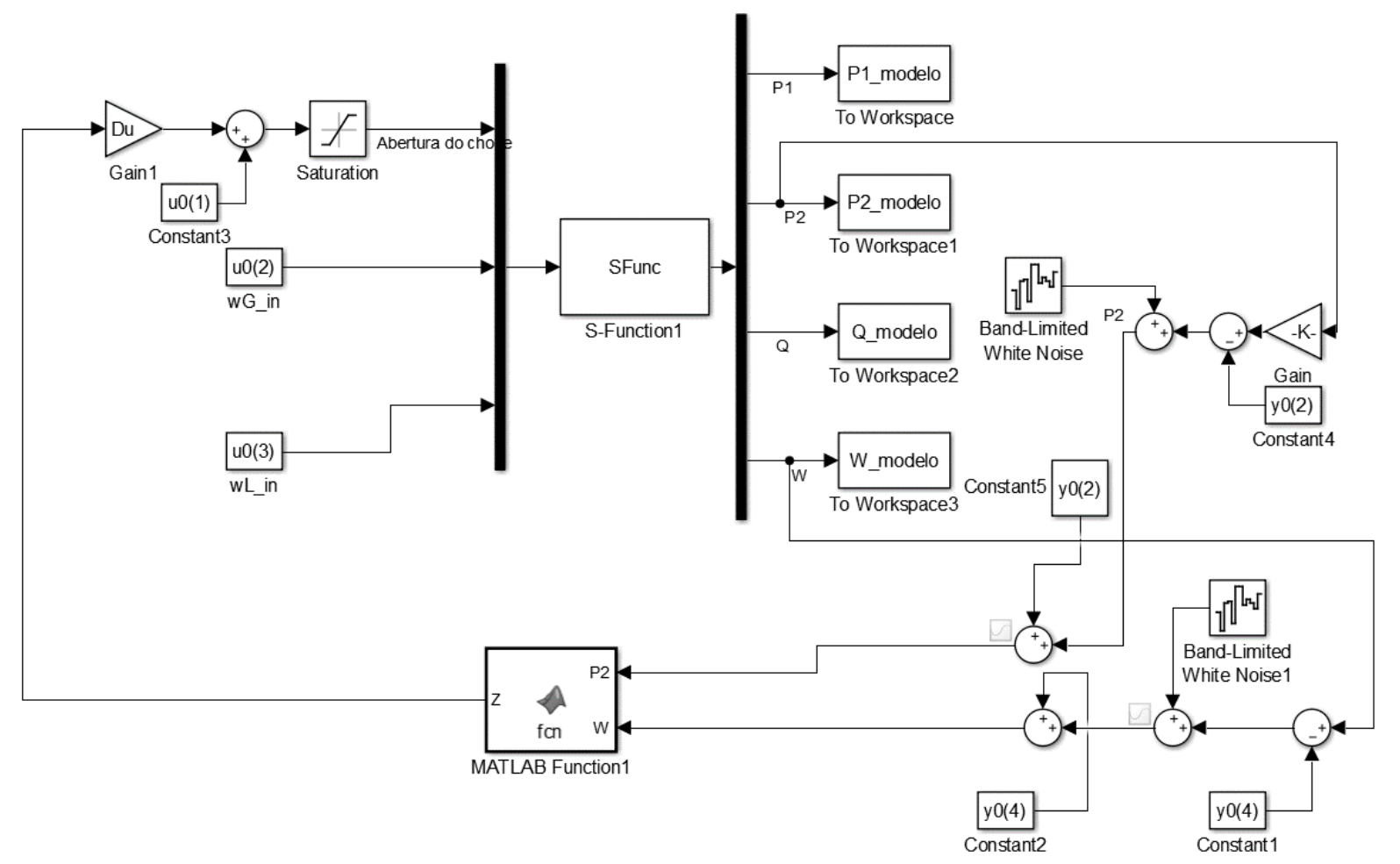

Figura C-1 - Diagrama Simulink do regulador linear quadrático (LQR) com Filtro de Kalman Unscented (UKF) utilizando o modelo linearizado do MATLAB

Posteriormente, realizou-se a implementação do sistema substituindo o modelo do MATLAB pela planta do OLGA ${ }^{\circledR}$. Para isto, utilizou-se blocos do Simulink para escrita e leitura de variáveis de forma a possibilitar a troca de informação entre os dois softwares conforme mostrado na Figura C-2. 


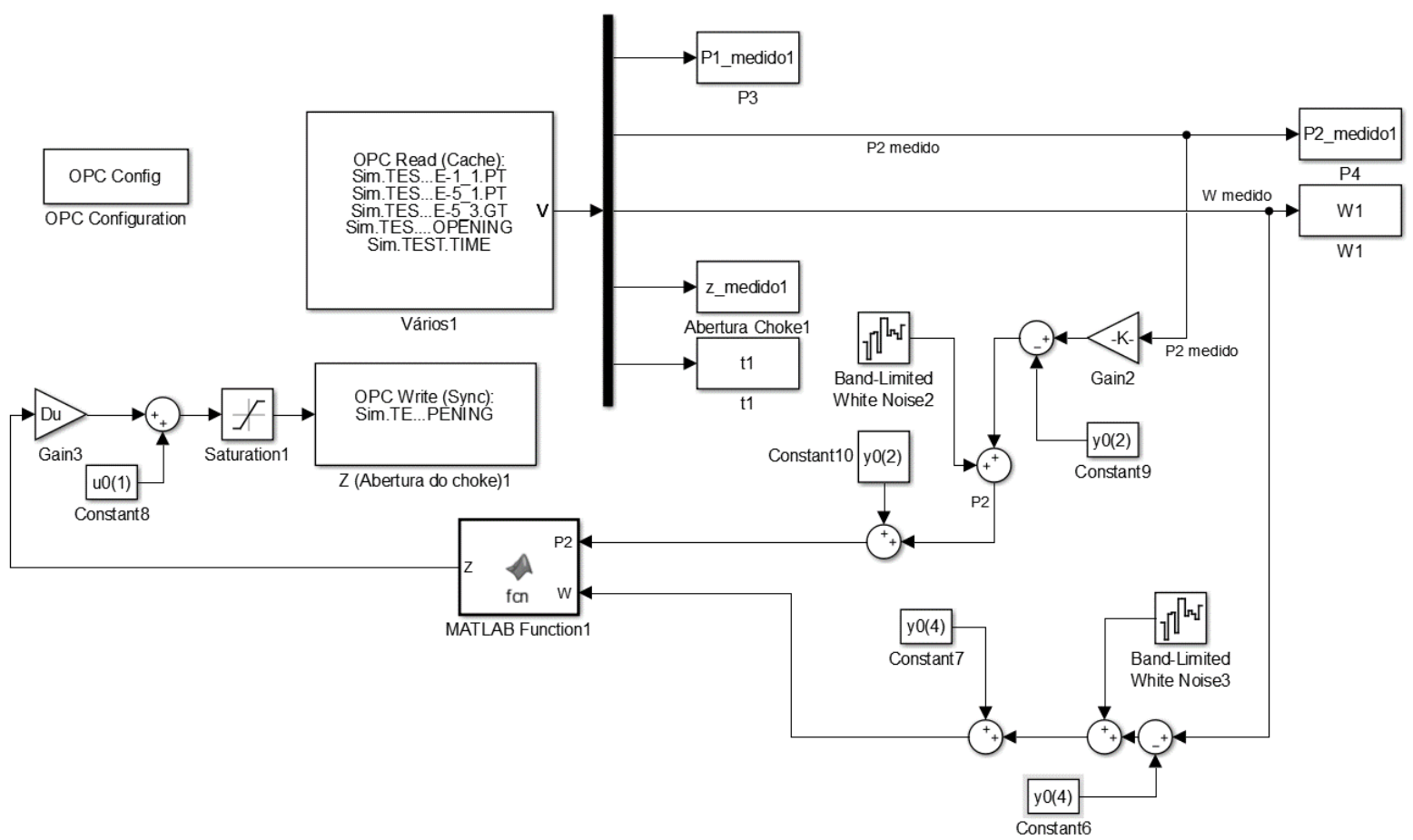

Figura C-2 - Diagrama Simulink do regulador linear quadrático (LQR) com Filtro de Kalman Unscented (UKF) utilizando o modelo do OLGA ${ }^{\circledR}$

\section{Código do MATLAB para o regulador linear quadrático}

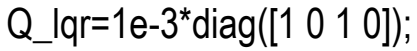

R_lqr=1e9;

N_lqr=zeros $(4,1)$;

$\left[\mathrm{K} \_l q r, S, e\right]=d l q r\left(F, B, Q \_l q r, R \_l q r, N \_l q r\right) ;$

U_c(k-1)=-K_lqr $x(:, k-1)$;

if( $\left(u_{-} c(k-1)<-u_{-} p c(k-1)\right)$;

u_c $(k-1)=-u_{-} p c(k-1)$;

end

if $\left(u_{-} c(k-1)>\left(1-u_{-} p c(k-1)\right)\right)$;

u_c(k-1)=1-u_pc(k-1);

end 
ANEXO A
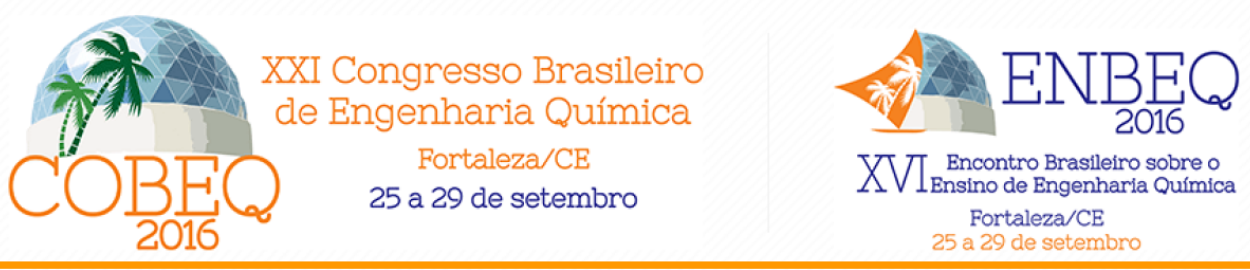

\title{
MITIGAÇÃO DE GOLFADAS NA PRODUÇÃO DE PETRÓLEO EM SISTEMAS OFFSHORE
}

\author{
F. H. ASHIKAWA ${ }^{1}$, S. W. PARK ${ }^{1}$ \\ ${ }^{1}$ Universidade de São Paulo, Escola Politécnica, Departamento de Engenharia Química \\ E-mail para contato: fabiohidashi@gmail.com
}

\begin{abstract}
RESUMO - Existem inúmeras instalações offshore operando em regimes instáveis de escoamento. Tal regime é resultado de diâmetros das linhas de produção superdimensionados ou baixa vazão dos poços no final da vida produtiva. Esta instabilidade traz dificuldades na produção offshore, pois pode causar danos nos equipamentos da plataforma ou perdas de produção. Neste âmbito, é necessário estudar meios de atenuar ou suprimir este tipo de regime de escoamento. $O$ objetivo deste trabalho é desenvolver métodos de forma a garantir a estabilidade do sistema através da modulação adequada da válvula choke, reduzindo ou mesmo eliminando o comportamento oscilatório da produção, mantendo a válvula choke na maior abertura possivel, trazendo desta forma, ganhos econômicos através do aumento da produtividade do poço. A análise do problema foi realizada através de simulações no MATLAB utilizando um modelo dinâmico simplificado encontrado na literatura. Este modelo foi escolhido, por reproduzir adequadamente o escoamento em golfadas severas, causado pela presença de trecho horizontal descendente na linha de produção. O problema foi também modelado no $O L G A \circledR$, sendo este, um software comercial com grande aplicação em simulações que exigem uma análise do comportamento transiente. Por fim, a avaliação da eficácia dos métodos de supressão das golfadas foi realizada através da integração entre o modelo do OLGA ${ }^{\circledR}$ e MATLAB via comunicação por servidor OPC.
\end{abstract}

\section{INTRODUÇÃO}

A exploração do petróleo se iniciou em jazidas de pequena profundidade que demandavam menores esforços e pouca tecnologia. Entretanto, à medida que estes campos superficiais se esgotaram, foi necessário um maior desenvolvimento tecnológico de forma a possibilitar a exploração de reservas mais profundas. No Brasil há um desafio adicional, pois, as maiores reservas de petróleo se encontram em alto mar (offshore). Com o avanço tecnológico, a fronteira exploratória se deslocou para lâminas d'água cada vez mais profundas.

Nestas condições, a tendência é que o escoamento de petróleo esteja sujeito a temperaturas

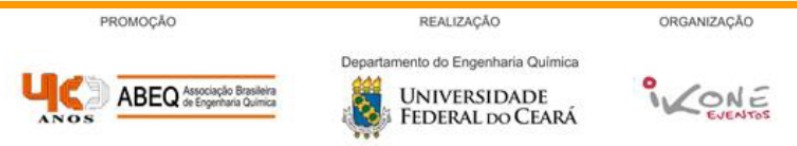



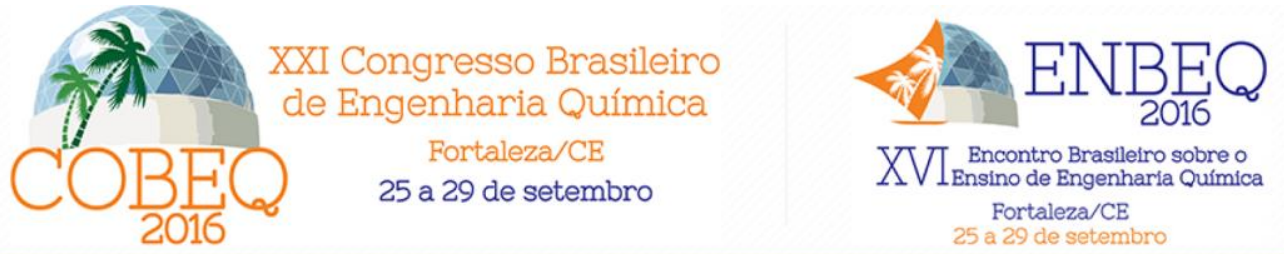

menores, o que possibilita a formação de bloqueios nas linhas pela formação de hidratos e/ou parafinas, além disso, há uma maior probabilidade de o escoamento ser intermitente (Jahanshahi, 2013).

Quando o regime de escoamento é caracterizado pela presença de golfadas, ou seja, é composto por severas variações na produção de óleo e gás, tem-se diversos problemas na plataforma, como: redução de eficiência na separação dos fluidos, possíveis perdas de produção ocasionadas por paradas não programadas (shutdown) ou restrição de produção por capacidade limitada da planta de processo, causada pelos picos de vazão dos poços.

Devido a estes desafios, o estudo do regime de escoamentos multifásicos na produção offshore e a mitigação de instabilidades é cada vez mais importante para se evitar perdas de produção e aumentar a produtividade dos campos de petróleo.

\section{MATERIAIS E MÉTODOS}

Ao estudar um escoamento em golfadas, o foco deve ser direcionado a capturar a dinâmica da formação da golfada, não a golfada em si, ou seja, o objetivo é evitar o regime de escoamento em golfadas ao invés de determinar o comprimento ou o formato das mesmas. Ao invés de lidar com modelos complexos, como os desenvolvidos no simulador OLGA ${ }^{\circledR}$, é necessário um modelo dinâmico simplificado para a análise de controlabilidade e projeto do controlador. Em Jahanshahi e Skogestad (2011), alguns dos principais modelos encontrados na literatura foram simulados no domínio do tempo e seus resultados, comparados ao modelo OLGA ${ }^{\circledR}$ com o objetivo de avaliar os cinco aspectos: abertura crítica da válvula choke para a formação de golfadas, frequência das oscilações com a válvula choke na abertura crítica, resposta dinâmica para uma mudança em degrau da abertura da válvula choke, pressão e vazão do sistema para o regime permanente e pressões e vazões mínima e máxima das oscilações durante o regime de golfadas.

Os modelos simplificados também foram analisados no domínio da frequência, onde foram consideradas a localização dos polos instáveis e zeros instáveis (RHP) do modelo. Ao final do comparativo, os modelos que apresentaram os melhores resultados e as melhores predições das pressões e vazões mínimas e máximas para o regime permanente quando comparadas aos resultados do OLGA $®$, foram o modelo proposto em Jahanshahi e Skogestad (2011) e o proposto em Di Meglio et al. (2009). Porém o primeiro apresentou um melhor ajuste para uma resposta em degrau da pressão de top-side

Neste trabalho, optou-se pela utilização do proposto em Jahanshahi e Skogestad (2011), pois considera-se que este é o modelo simplificado mais representativo do sistema real e apresenta vantagens sobre os demais.

PROMOChA



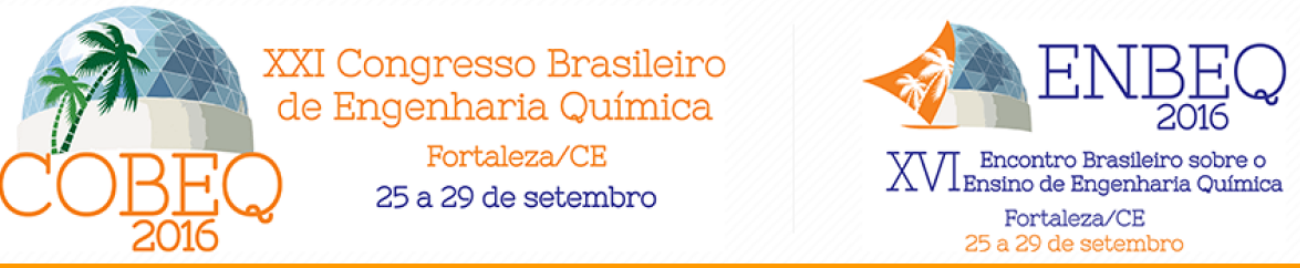

\subsection{Modelo Dinâmico Simplificado}

O diagrama simplificado para o escoamento sem bloqueio de líquido na base do riser é mostrado na Figura 1 e o diagrama simplificado para o escoamento com bloqueio de líquido, levando a um regime de golfadas severas está mostrado na Figura 2 (Jahanshahi e Skogestad, 2011).

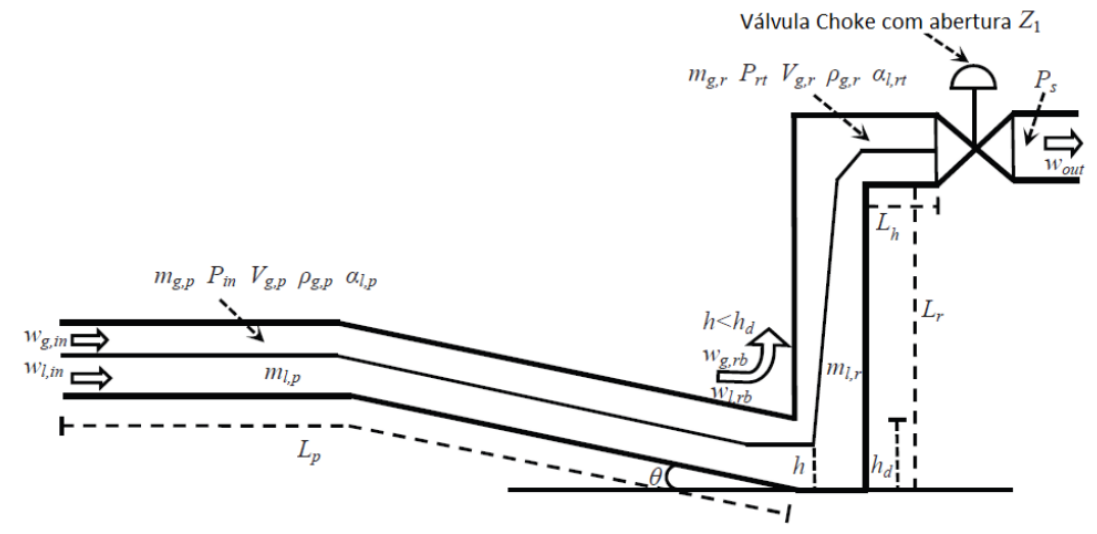

Figura 1 - Representação simplificada do escoamento desejado (Sem bloqueio de líquido).

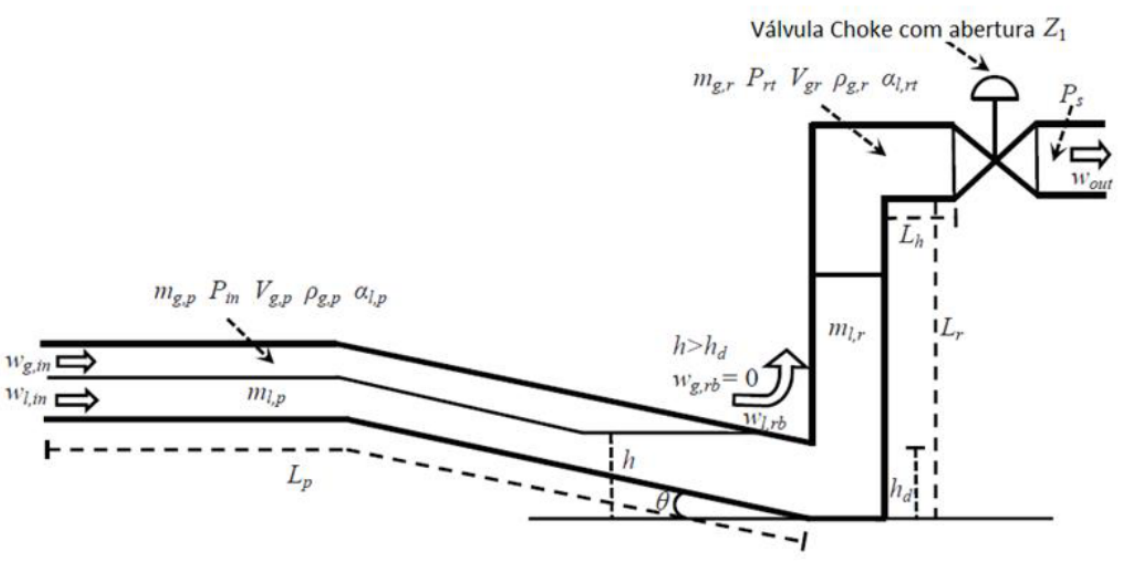

Figura 2 - Representação simplificada do bloqueio de líquido na base do riser,

Nesta modelagem, as vazões mássicas de entrada de gás e de líquido são consideradas constantes e as vazões mássicas de saída para uma mistura bifásica são dadas pela equação da válvula choke mostrado pela Equação 1.

$w_{o u t}=C_{v 1} \cdot f\left(z_{1}\right) \sqrt{\rho_{r t} \cdot \max \left(P_{r t}-P_{s}, 0\right)}$

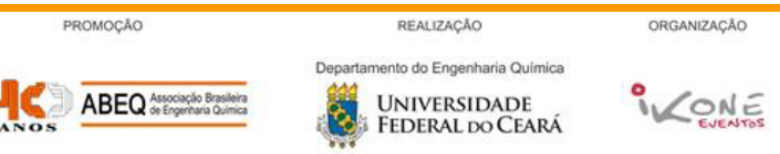




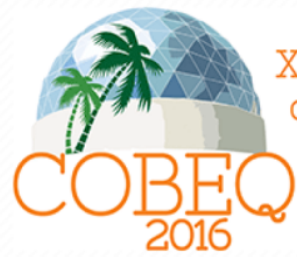

XXI Congresso Brasileiro de Engenharia Química

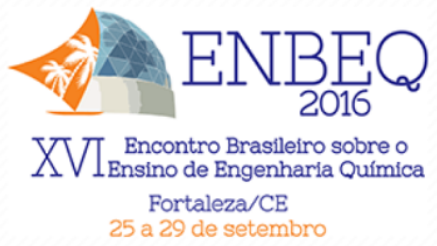

Quanto o líquido não está bloqueando a base do riser $(\mathrm{h}<\mathrm{hd})$ conforme Figura 1 , o gás irá fluir através da mesma e a modelagem pode ser dada por uma "equação de orifício" conforme mostrado na Equação 2:

$w_{g, r b}=K_{g} \cdot A_{g} \cdot \sqrt{\rho_{g, p} \cdot \Delta P_{g}}, \quad h<h_{d}$

A dinâmica do bloqueio de líquido ocorre quando o nível de líquido ultrapassa a abertura do duto na base do riser ( $\mathrm{h}>\mathrm{hd}$ ), momento no qual, o líquido causa um bloqueio do gás, ou seja, a vazão de gás na base do riser é zero, conforme mostra a Figura 2.

\subsection{Modelagem do Sistema no Simulador OLGA®}

Um sistema sujeito a golfadas severas foi modelado utilizando o software OLGA ${ }^{\circledR}$ conforme Figura 3. Para esta modelagem, considerou-se alguns parâmetros geométricos de um sistema fictício mostrados na Tabela 1.

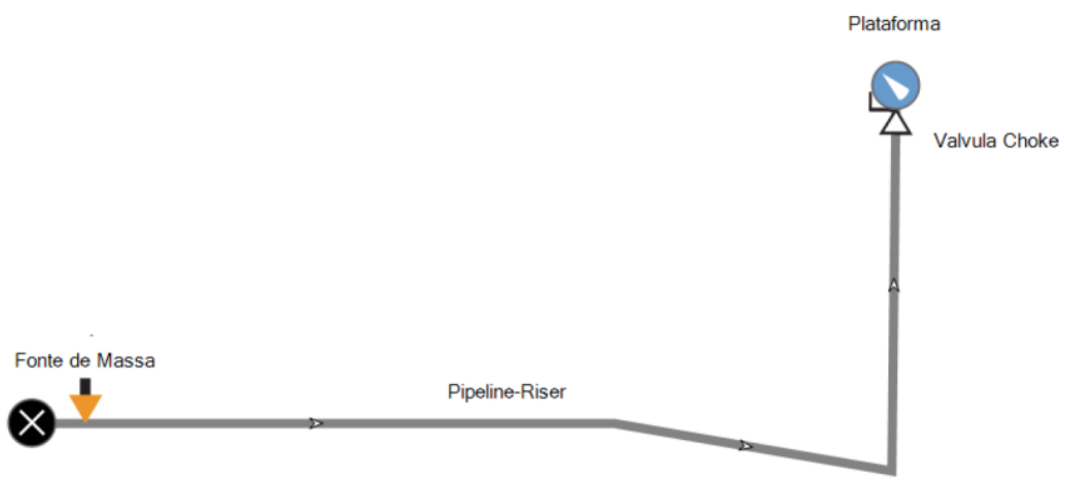

Figura 3 - Modelo do OLGA® para o sistema pipeline-riser.

Tabela 1 - Dados da geometria do sistema modelado no OLGA®.

\begin{tabular}{ccccc}
\hline Trecho & Diâmetro $(\mathrm{m})$ & Rugosidade $(\mathrm{m})$ & Comprimento $(\mathrm{m})$ & Elevação $(\mathrm{m})$ \\
\hline PIPE-1 & 0.12 & $2.8 \mathrm{E}-05$ & 2000 & 0 \\
PIPE-2 & 0.12 & $2.8 \mathrm{E}-05$ & 2000 & -34.9 \\
\hline
\end{tabular}

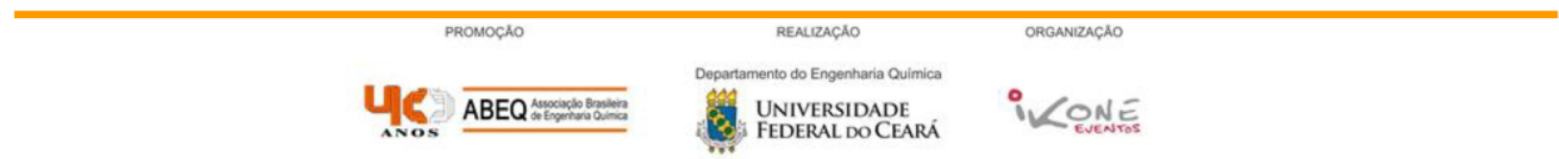




\begin{tabular}{ccccc}
\hline PIPE-3 & 0.12 & $2.8 \mathrm{E}-05$ & 300 & -5.24 \\
PIPE-4 & 0.1 & $2.8 \mathrm{E}-05$ & 300 & 300 \\
PIPE-5 & 0.1 & $2.8 \mathrm{E}-05$ & 100 & 0 \\
\hline
\end{tabular}

\subsection{Controlador PI com Ganho Adaptativo}

A implementação deste controlador foi realizada com base no ganho estático do sistema e na abertura média da válvula choke (Jahanshahi, 2013). O ganho estático do sistema em função de z, assumindo que a válvula possui abertura linear é dado pela Equaçao 3.

$k(z)=\frac{-2 \bar{a}}{z^{3}}$

Onde z é a abertura do choke e varia de $0 \leq \mathrm{z} \leq 1$.

Os valores do ganho proporcional e do tempo integral podem ser escritos em função da abertura da válvula choke (z) e são dados respectivamente pelas Equações 4 e 5.

$k_{c}(z)=\frac{\beta \cdot T_{o s c}}{k(z) \cdot \sqrt{z / z^{*}}}$

$\tau_{I}(z)=3 \cdot T_{o s c} \cdot\left(\frac{z}{z^{*}}\right)$

Onde $z^{*}$ é a abertura crítica da válvula choke do diagrama de bifurcação.

\section{RESULTADOS}

Simulou-se o comportamento das pressões de entrada (linha preta) e saída (linha vermelha) para uma abertura da válvula choke em rampa (entre 2\% e 100\%) conforme Figura 4 . O objetivo é determinar a abertura crítica, a partir da qual, as pressões da entrada e de saída se tornam instáveis e iniciam o comportamento oscilatório. Através desta análise, nota-se que as instabilidades se iniciam para aberturas da válvula choke em $7 \%$, sendo esta, a abertura crítica. Através desta simulação, foi possível determinar também, de forma indireta, o diagrama de bifurcação do sistema, visto que os ranges de oscilação para aberturas da válvula choke acima da crítica, são bem visíveis.

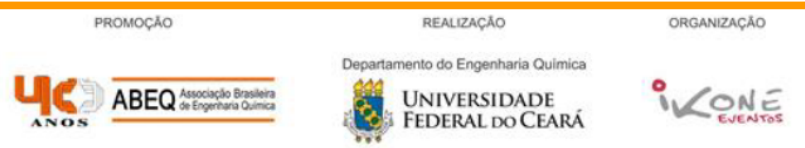



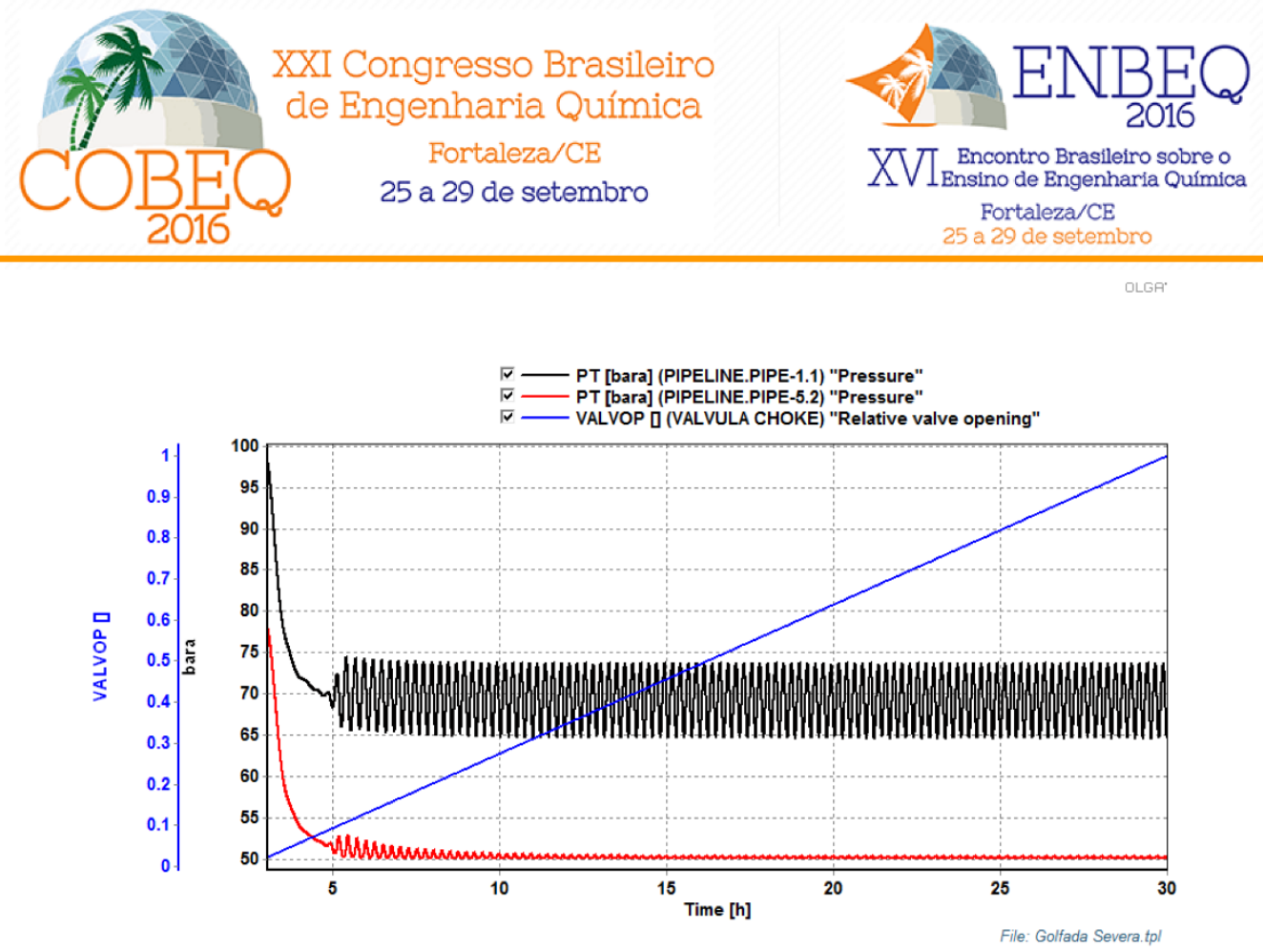

Figura 1 - Oscilação das pressões de entrada e saída para diferentes aberturas da válvula choke.

Considera-se que o modelo acima representa o sistema real. Então modelou-se o sistema simplificado no MATLAB, conforme descrito na seção 2.1, a partir do qual foram plotados os diagramas de bifurcação das pressões de entrada e saída conforme Figura 5 onde a linha tracejada representa a região de equilíbrio instável, sendo esta, o ponto ideal de operação onde é possível elevar a produtividade, visto que esta região representa uma pressão menor que a média das pressões máximas e mínimas das oscilações.
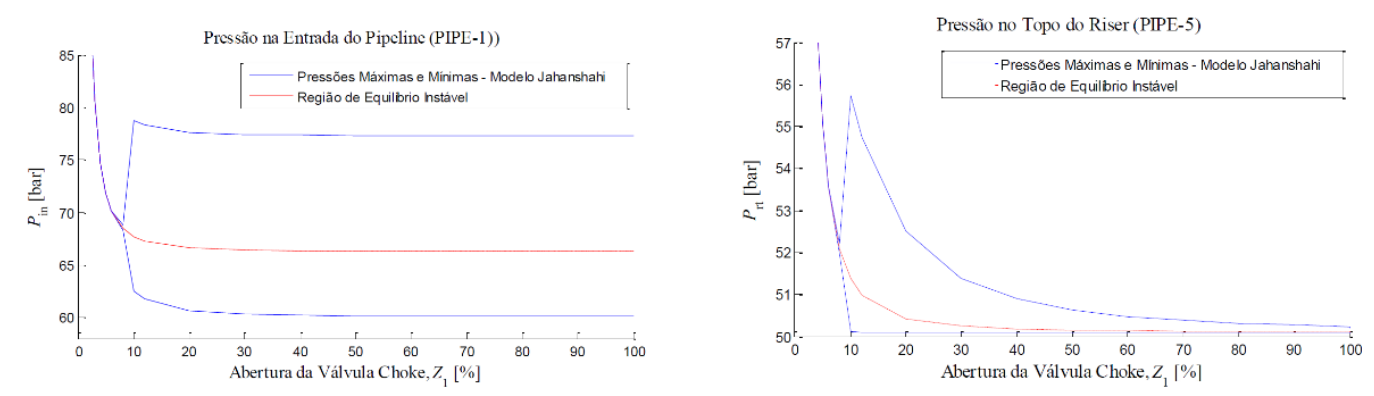

Figura 2 - Diagrama de bifurcação - Pressões de entrada e saída x abertura da válvula choke.

O sistema proposto é instável para aberturas fixas da válvula choke superiores a abertura crítica, portanto, para se operar na região instável, aumentando a produtividade do poço e mitigando as golfadas, é necessário modular adequadamente a válvula.

Utilizou-se neste trabalho, o controlador PI com ganho adaptativo citado na seção 2.3 , de forma a lidar melhor com as variações da planta e com diferentes pontos de operação.

\section{PROMOCAO \\ ORGANIZACAAO

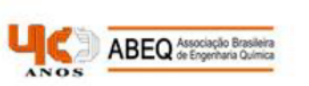 \\ UNIVERSIDAD \\ UNIVERSIDADE



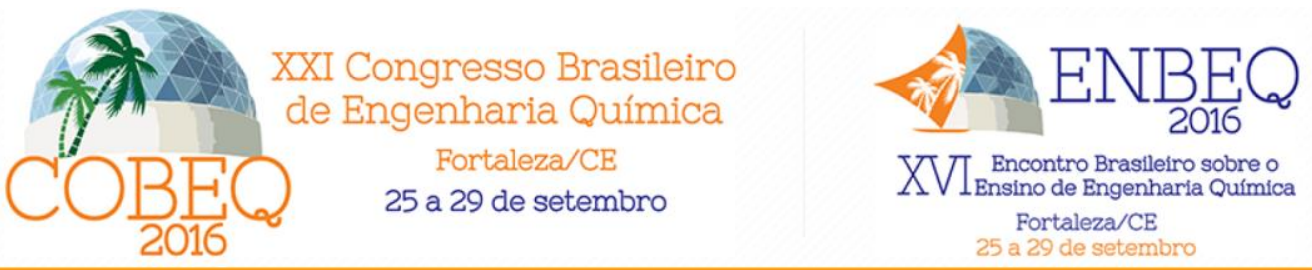

A implementação do controlador foi realizada no Simulink ${ }^{\circledR}$ e o mesmo foi testado no OLGA ${ }^{\circledR}$ em tempo real através da comunicação via servidor OPC entre o OLGA ${ }^{\circledR}$ e o MATLAB mostrado na Figura 6.

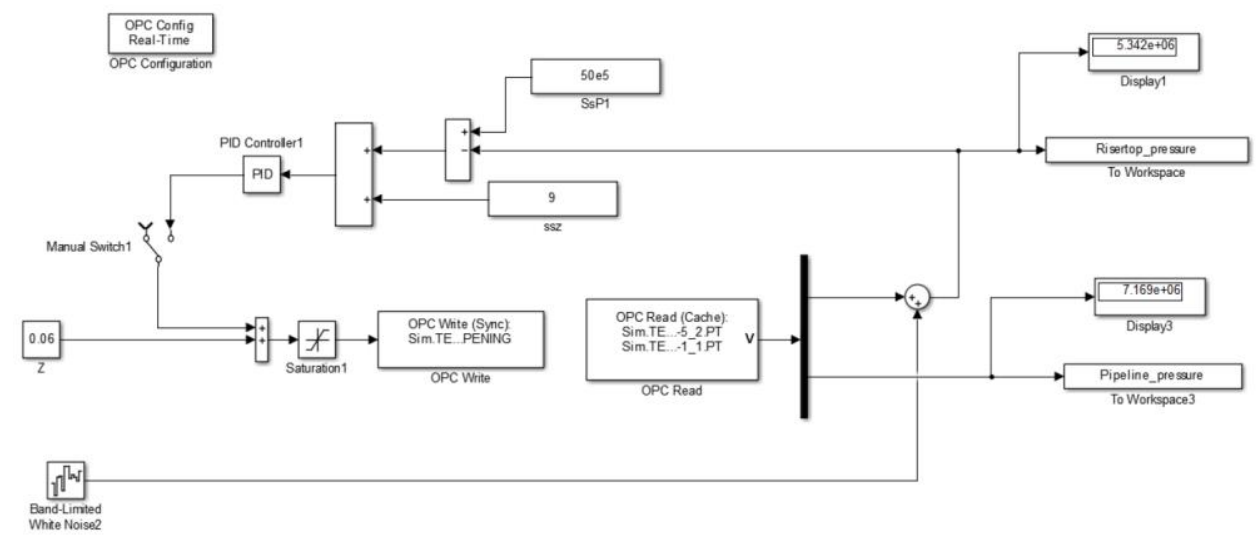

Figura 6 - Modelo simulink ${ }^{\circledR}$ para comunicação OPC entre o MATLAB e o OLGA ${ }^{\circledR}$

Como variável controlada, foram utilizadas as pressões de entrada (fundo do poço) e de saída (topo do riser), pois as mesmas são as mais comumente encontradas nos sistemas reais, seja através de sensores de pressão submarinos ou na plataforma e como variável manipulada, foi utilizada apenas a válvula choke no topo do riser.

A Figura 7 mostra a pressão de entrada após a implementação do controlador PI com ganho adaptativo, proposto na seção 2.3. Nota-se que o mesmo proporciona um range de oscilações muito menores da pressão citada, proporcionando também uma pressão média menor, trazendo desta forma, ganhos de produtividade ao poço.

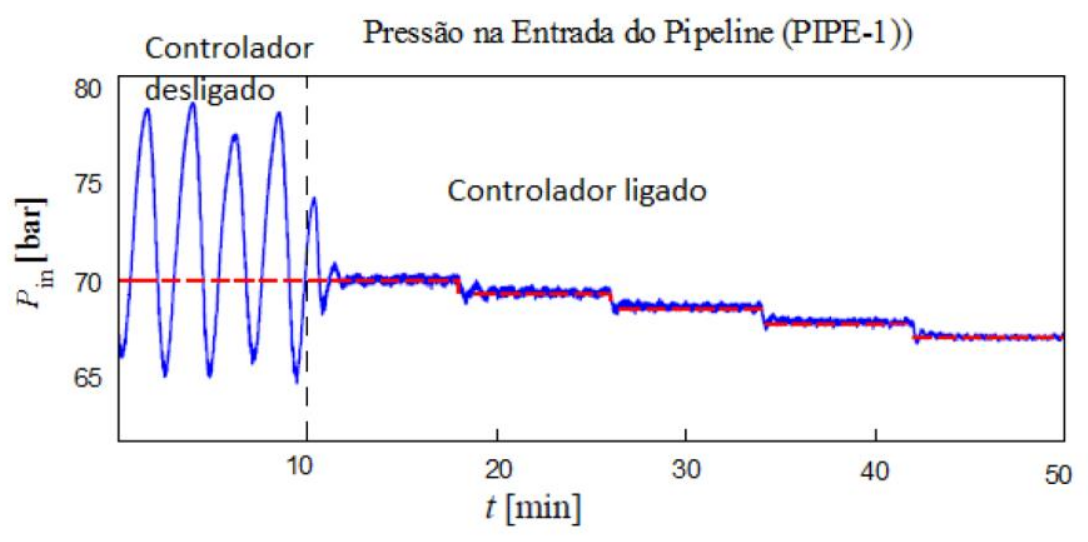

Figura 7 - Pressões de entrada para o controlador adaptativo.

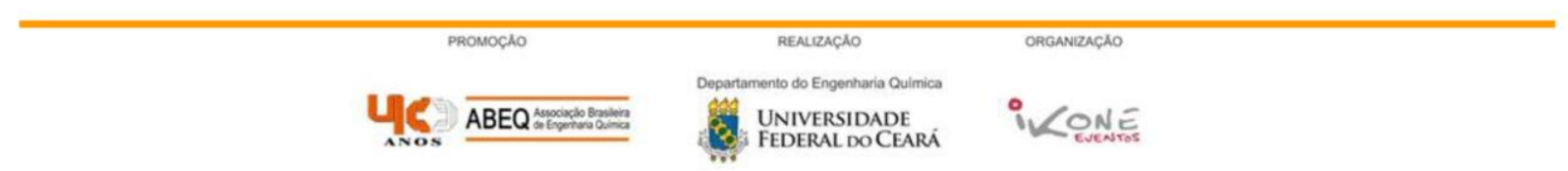



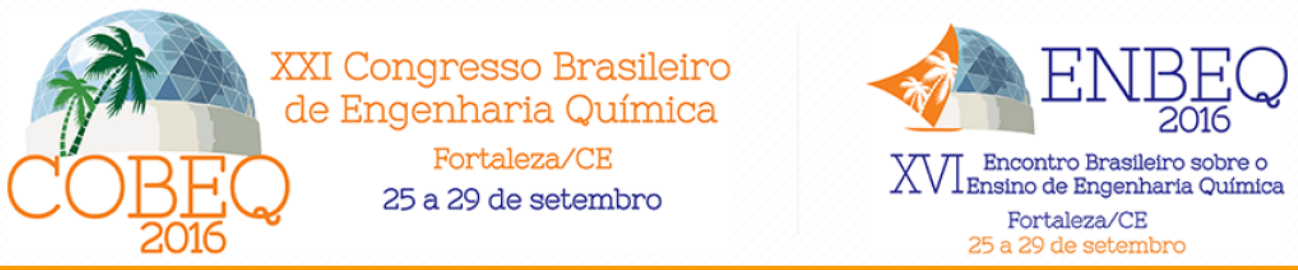

\section{CONCLUSÕES}

Através da comparação dos resultados apresentados na seção 3, é possível avaliar se o modelo desenvolvidos no MATLAB se ajustam bem a simulação do OLGA ${ }^{\circledR}$. Apesar das diferenças verificadas no diagrama de bifurcação, considera-se que o modelo proposto em Jahanshahi e Skogestad (2011) implementado no MATLAB apresenta bom ajuste com os resultados das simulações no OLGA ${ }^{\circledR}$. Com base em tais resultados, o modelo simplificado foi utilizado para o desenvolvimento de algoritmos de controle, com o objetivo de suprimir golfadas nos pontos de operação em que o sistema é instável (para aberturas da válvula choke maiores que a abertura crítica).

Para o controlador PI com ganho adaptativo, nota-se que a pressão de entrada oscila em um range muito menores, o que indica regime de produção mais estável, adicionalmente, a pressão média também é visivelmente menor, trazendo desta forma, ganhos de produtividade ao poço.

\section{REFERÊNCIAS}

JAHANSHAHI, E.; SKOGESTAD, S. Simplified Dynamical Models for Control of Severe Slugging in Multiphase Risers. 18th IFAC World Congress, 2011.

JAHANSHAHI, E. Control Solutions for Multiphase Flow Linear and nonlinear approaches to antislug control. Tese de Doutorado, 2013.

MEGLIO, F. D.; KAASA, G. O.; PETIT, N. A first principle model for multiphase slugging flow in vertical risers. Joint 48th IEEE Conference on Decision and Control and 28th Chinese Control Conference, 2009.

\section{PROMOCGAO REALZAÇAO ORGANIZACAOAO

\begin{tabular}{|c|c|}
\hline & \\
\hline 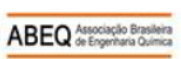 & GA UNIVERSIDADE \\
\hline
\end{tabular}


ANEXO B

Available online at www.sciencedirect.com

ScienceDirect

IFAC-PapersOnLine 48-6 (2015) 083-088

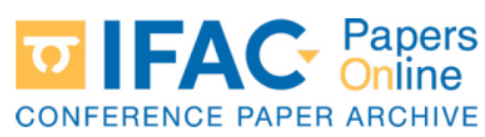

Advanced Anti-slug Control for Offshore Production Plants

\author{
Campos, M. ${ }^{1}$, Takahashi, T. ${ }^{2}$, Ashikawa, F. $^{3}$, Simões, S. $^{4}$, Stender, A. ${ }^{5}$, Meien, O. $^{6}$ \\ Petrobras, Research Center-CENPES,UO-RIO,UO-BS, Brazil, (e-mail: 'mariocampos@ petrobras.com.br, \\ ${ }^{2}$ thiagotakahashi@petrobras.com.br, ${ }^{3}$ fabio_ashikawa@petrobras.com.br, ${ }^{4}$ saulsimoes@ petrobras.com.br, \\ 5alberto.santanna@petrobras.com.br ${ }^{6}$ meienov@petrobras.com.br, )
}

\begin{abstract}
The application of advanced control techniques in offshore oil production plants is a challenge. There are many changes in operational points in time, for example, the process is affected by the natural oil well's behavior dynamics. Besides that, limited instrumentation available has to be considered when thinking in oil optimization and control. In order to improve the scenario, taylor-made advanced control modules have been developed for those units. The present article will present development, implementation and results of anti-slug control for three platforms located at Campos and Santos basins. The controllers were designed to achieve improvement in operational stability and safety, decreasing in unscheduled compressor shutdown events, as well as increasing in operational efficiency.
\end{abstract}

(C) 2015, IFAC (International Federation of Automatic Control) Hosting by Elsevier Ltd. All rights reserved.

Keywords: slug control, advanced control, offshore platform's automation, industrial control and results.

\section{INTRODUCTION}

Severe slugging is a common problem in many offshore platforms, because production facilities cannot handle the large flow and pressure variations due to slugging. As a consequence, unstable flow results in poor separation that may cause damage to critical equipment like heat exchangers, increases maintenance costs, and may also cause unscheduled shutdowns due to unstable multiphase flow. So, high pressure trips in compressors or high level trips in separators are able to generate substantial economic losses. There are many different mechanisms which cause unstable flow, or slugs, depending on flowline geometry, fluid distribution among phases and equipment. Slugging most common causes are due to irregularity in the sea bed terrain, vertical pipeline sections, instability in gas-lift flow, differences in gas/liquid velocities, etc. A description of slug mechanisms can be found in Pickering et al. (2001), Hu (2004), Sinegre (2006) and Kaasa et al. (2007).

The traditional method of minimizing slugs is manually choking flow at the expenses of production decreasing, increasing gas-lift flow, and subsea chemical injection, both leading to costs increasing. Another option is to use automatic control to reduce or even eliminate oscillations, without production loss, manipulating the choke valve (Storkaas, 2005) (Storkaas and Skogestad, 2007). Therefore, it is important to continue developing new control strategies which result in attenuation or protection against severe slugging.

In practice, there is some resistance by operators in using new automatic control, particularly anti-slug control, due to the belief of keeping choke valve fully open will result always in production improvement. Which is true for wells with no slug pattern, however may cause production losses in wells with slugging flow (Hu, 2004).

According to the American Petroleum Institute, advanced control is defined as any control strategy that has functions beyond those commonly associated with regulatory control. In this paper, it is discussed an advanced anti-slug control strategy, which can be thought as an expert system with three modules: slug diagnostic, anti-slug control algorithm, with auto-tuning capabilities, and severe slugging protection. This system was implemented on a process computer that communicates with the platform's automation system.

Although advanced control systems are reality in many industrial areas like refineries, petrochemicals and gas plants (Campos et al., 2009), they are not widely used for offshore production units (Campos et al., 2013). Many reasons can be pointed as causes for such behavior: no advanced control specialist onboard, more transients and disturbances due to oil well behavior, uncertainties and noise, non-linear and time-varying process, lack of instrumentation, etc.

Due to these problems, linear and multivariable predictive advanced controllers, common in other areas such as refineries and petrochemicals are difficult to apply in platforms' process. Thus, different advanced control strategies were proposed and applied to offshore platforms. The problem was divided in small and less complex problems. Each one has an expert system to deal with. One of the goals was to develop anti-slug control for wells, which will be the focus of this paper. This system has a control algorithm, with some degree of adaptation for each different operational condition, and also has some modules to diagnose and protect process equipment. The advanced controller was developed and implemented in three offshore platforms and 
the results will be shown in the following discussion. The implementations of anti-slugs advanced control strategies bring the following benefits:

- Reduction in production losses due to unplanned shutdowns.

- Increasing stability and profitability.

- Increasing safety and operating reliability.

- Increased sustainability by minimizing flaring.

In this article, we will present some details about advanced anti-slugging control strategy, results and economic gains obtained with its implementation in oil production platforms.

\section{SLUGS PROBLEM DESCRIPTION}

A scheme of unstable multiphase flow cyclic behavior is shown in Figure 1. This is an example of severe slugging caused by pipeline-riser systems with low points in front of the riser, where "slugs" of liquid accumulate before pushed upwards by gas. In the first sub-figure, liquid blocks the low point of the riser preventing gas flow. Liquid falls back from the riser by gravity and causes the slug to grow and fill the riser. The pressure in the pipeline steadily increases due to the inlet flow of gas until it is large enough to push the liquid slug out of the riser causing a great disturbance to downstream separators. When the tail of the liquid slug enters the riser, downhole pressure drops due to the reduced static head of the liquid column which causes the gas to expand. When gas leaves the riser, a large disturbance is generated to compressors pressure and anti-surge controllers. After that, velocities in the riser become too low to carry liquid up the riser and process starts again with liquid accumulation, fallback, in lower points.

Slugs represent a major challenge to downstream processing facilities due to large variations in flow and pressure. As pointed before, the possible consequences are: unscheduled shutdowns, damage to topside equipment, production decreasing, resulting in substantial economic losses and increase in maintenance costs.

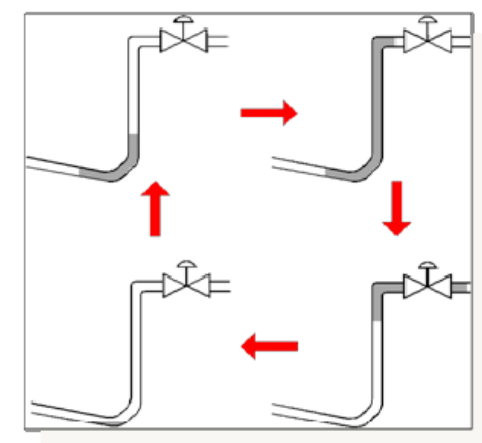

Fig. 1. Slug cycles.

One way to eliminate slugs is manually choking flow until reach stability; however this method has the drawback of production loss. Another option is the use of automatic control to reduce, or even eliminate, oscillations. In figure 2 , it is shown the oscillating behavior of the downhole pressure (PDG - Permanent Downhole Gauge) of a slugging well. There is a maximum valve opening which enables stable operation when no control is applied. If we try to operate above bifurcation point unstable multiphase flow occurs characterized by the stable limit cycle where downhole pressure oscillates between high and low pressure values. The upper red line of the figure 2 shows the maximum pressure at a particular valve opening and the red lower line the minimum pressure. The dashed line in the middle shows the unstable steady-state solution, which is the desired operating point in closed-loop operation.

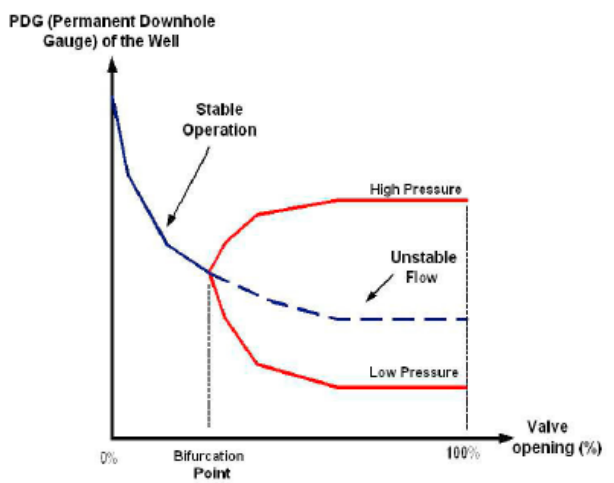

Fig. 2. Bifurcation plot (red line shows the limit cycle).

\section{ANTI-SLUG ADVANCED CONTROL}

The proposed advanced anti-slug control has three main modules, as shown if figure 3 :

- Diagnostic Module - responsible for detecting severe slugs based on pressure measurements.

- Anti-slug Protection Module - responsible for preventing propagation of severe slugs to topside equipment (separators and compressor).

- Anti-slug Control Module - responsible for minimizing or even eliminating slugs. If possible, keeping choke valve at the desired position.

Following these modules will be described.

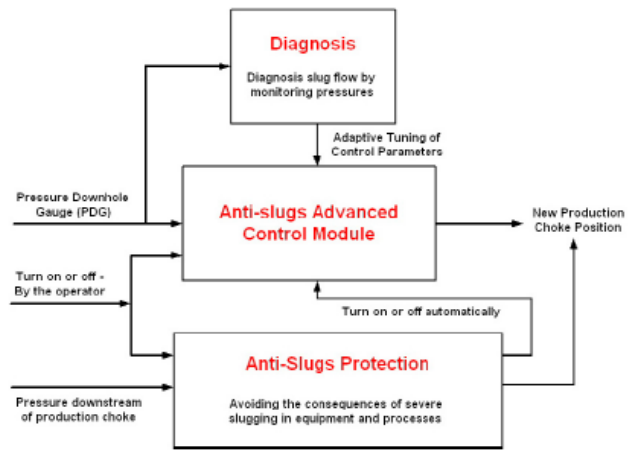

Fig. 3. Block diagram of the Anti-slug Advanced Control. 


\subsection{Diagnostic Module}

This module tries to detect oscillations in the available pressure gauges: downhole pressure (PDG), wet Christmas tree pressure (TPT - Temperature and Pressure Transducer) and upstream choke pressure. Pressure is considered to be oscillating if the difference between maximum and minimum values within a time window is above a fixed control parameter. This information will be used by the anti-slug control module, to adjust its tuning parameters, and by the protection module.

\subsection{Anti-Slug Protection Module}

The Anti-slug Protection Module is designed to prevent the propagation of severe disturbances to topside equipment. If there is measurement of pressure downstream choke, this value is used and compared with a maximum desired parameter. If there is only pressure measurement upstream choke, we have to infer the maximum pressure allowed downstream for a particular choke valve position, as shown in figure 4 . We use historical process data, which has great variability, to obtain or tune this curve in order to protect equipment (red line in the figure 4).

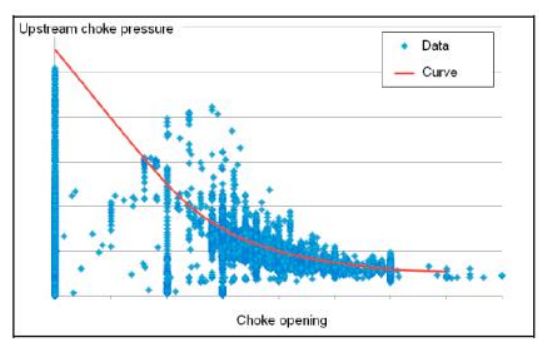

Fig. 4. Protection curved - Upstream choke pressure as a function of the choke opening (\%).

Based on the inferred curve, the protection algorithm determines the maximum pressure value. If measured pressure remains above this threshold during a certain number of control cycles, choke is closed to a minimum value defined in the operators interface. The protection algorithm will maintain the choke valve at its minimum value while it detects severe slugs. When pressure becomes stable and below the maximum value, system allows the anti-slug control module to open again the choke valve.

\subsection{Anti-slugs Control Module}

The most common anti-slug control strategy uses downhole pressure as controlled variable of a PID algorithm and the choke opening as the manipulated variable (Dalsmo et al., 2002), (Storkaas et al., 2003), (Godhavn et al., 2005), (Stasiak et al., 2012). The objective is to eliminate or at least to minimize slug disturbances to the process. However, in practice many wells can lose their downhole measurements due to problems in the sensor or in the communications cables. Therefore, it was necessary to develop new algorithms using only topside pressure. We will describe briefly some algorithms that are available for being chosen for a specific application in a platform.

\subsubsection{ONFC (On-line Neuro-Fuzzy Controller) Algorithm}

The ONFC algorithm (Gouvêa, 2005), (Carvalho, 2010), (Carvalho et al., 2010) is used instead of the PID controller because it can deal with nonlinear process and it has adaptive characteristics. The setpoint of the ONFC is defined by another controller that tries to find out the downhole pressure which results in a desired choke position defined by operator. Figure 5 shows the block diagram of this algorithm.

In this ONFC algorithm the controller output (u) is obtained by error's membership functions and some tuning parameters (w - weights), which were adapted depending on the error.

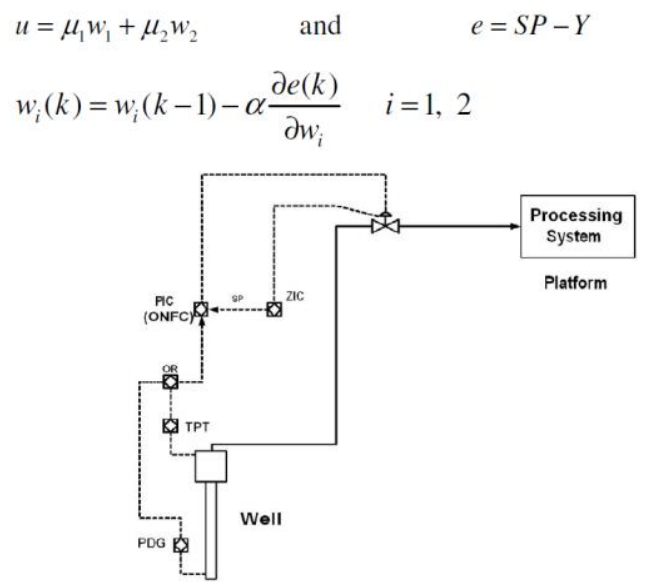

Fig. 5. Flowchart of the ONFC Algorithm.

\subsubsection{Three (3) PIDs Algorithm}

This algorithm is based on three PIDs controllers that compete with each other in override strategy to handle the choke opening. The controlled variables are: PDG or TPT, upstream and downstream choke pressures. This logic, like the ONFC algorithm, also depends on the right choice of the setpoint for each pressure controllers. Figure 6 shows a flowchart of this algorithm.

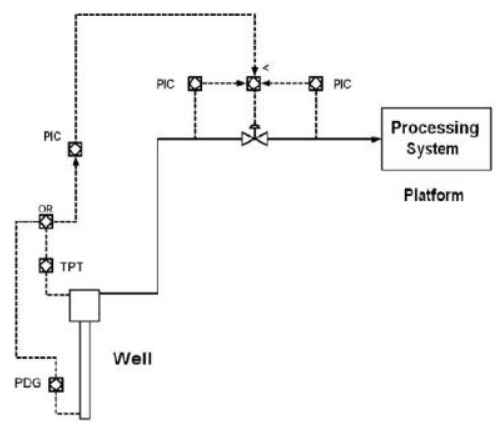

Fig. 6. Flowchart of the 3 PIDs Algorithm. 


\subsubsection{Gamma Algorithm}

In order to avoid the problem of defining the right pressure setpoint for each operating condition, it was developed a Gamma algorithm, which is based on work published by Stasiak, Pagano and Plucenio (2012). The control purpose is to suppress slug oscillations ensuring stable system operation and, at the same time, to keep the control at a desired choke position value. Gamma algorithm uses the choke valve opening as the output variable, instead of pressure setpoint as in Stasiak et al. (2012). The control law is given by:

$$
\Delta U_{k+1}=\gamma_{1} \times \Delta P D G_{k}+\gamma_{2} \times \Delta P D G_{k-1}+\beta \times\left(S P_{C H O K E}-U_{k}\right)
$$

where $S P$ is the desired choke operating point $(U)$ and the parameters $\gamma$ are tuning values to obtain a desired anti-slug performance (stabilizing action), and $\beta$ is another tuning parameter that adjusts how fast the control action reaches the desired operating setpoint. The tuning of these parameters should take into consideration that the stabilization process pressure is a priority. This algorithm can be used to control any of the following variables: downhole pressure (PDG or TPT), upstream choke pressure or downstream choke pressure.

\subsubsection{Period Algorithm}

The goal of this algorithm is to identify the characteristics of the slugs (period and amplitude) in order to predict future slugs and close or open the choke valve at the right moment. This algorithm was developed to use downhole pressure, or upstream choke pressure. Figure 7 shows a schematic of this period algorithm.

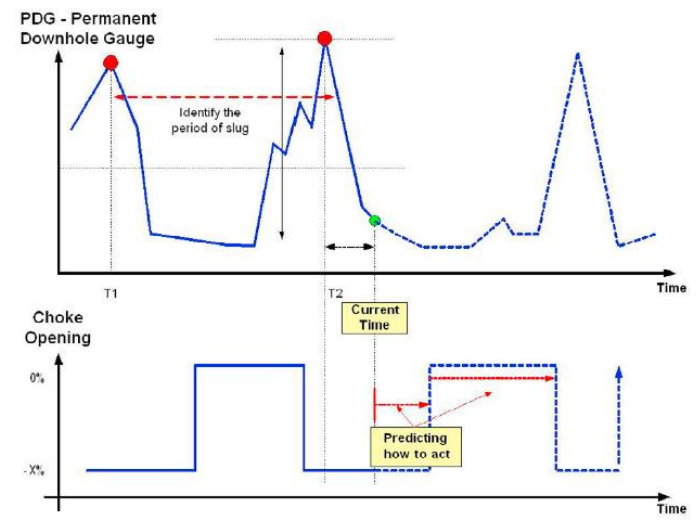

Fig. 7. Period algorithm actuation.

\section{ANTI-SLUG CONTROL IMPLEMENTATION}

The algorithms presented in this paper were implemented in the MPA environment (Module of Automated Procedures), which is a tool developed by Petrobras and PUC University to implement advanced automation and control strategies. Figure 8 shows part of the anti-slug advanced control implementation in the MPA software. The controller is executed every 10 seconds. This tool uses a flowchart language that is easy to implement and to maintain the control rules. MPA runs in a process computer and communicates with the platform's automation system through a driver or an OPC protocol.

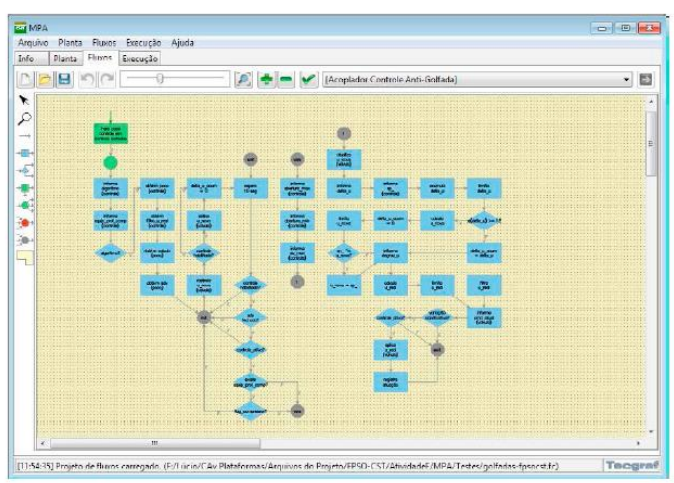

Fig. 8. Example implementation of the MPA

\section{RESULTS AND ECONOMIC GAINS}

This anti-slug advanced control was implemented in three offshore oil production platforms. In one platform, due to the limitation of the choke valve actuator, it was used only the anti-slug protection module. In the Anti-slugs Control Module, the Gamma Algorithm was chosen for the implementation in the platforms, because it was easier to tune and does not require setpoints. For some wells, we use the PDG as process variable, but there were other wells where this information was not available. As a consequence, pressure upstream choke was used as input for anti-slug controller with acceptable performance.

Table 1 shows the performance of the controller for three platforms. It can be seen that the system was able to reduce the time that pressure downstream choke was larger than maximum limit for all platforms. This is related with the performance of protection module, showing that this control system increased the operational safety.

Table 1. Time (\%) with header pressure above a limit.

\begin{tabular}{|c|c|c|c|}
\hline & Before & After & Reduction \\
\hline Platform A & 0,65 & 0,05 & $-92 \%$ \\
\hline Platform B & 0,09 & 0,02 & $-78 \%$ \\
\hline Platform C & 1,92 & 1,25 & $-35 \%$ \\
\hline
\end{tabular}

Figure 9 shows an example of operation of the anti-slug advanced control for one well (with protection and control algorithm). As we can see, there was a decrease in variation amplitude of upstream choke pressure (green line) when the anti-slug control was manipulating choke opening (controller in automatic mode). The yellow line in this figure is the pressure of the oil separator. 
Table 2 quantifies the reductions in the amplitude and the average value of the subsea pressure (TPT) for one well. There was an increase in operational stability due to the decrease in TPT pressure amplitude and increase in production due to the reduction of its average value.

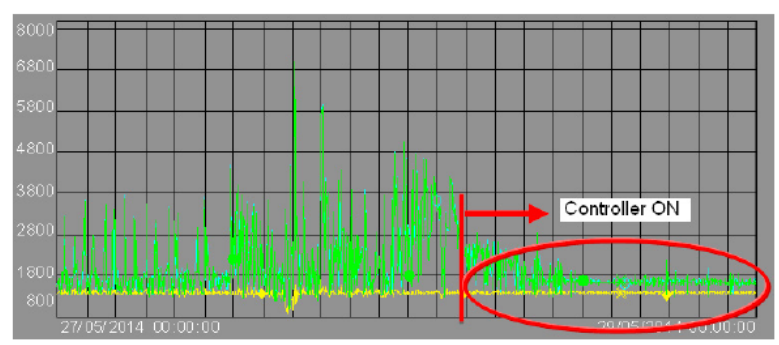

Fig. 9. Performance of the anti-slug control for one well.

Table 2.Effect on TPT due to Anti-slug Advanced Control

\begin{tabular}{|c|c|c|c|}
\hline & Before & After & Reduction \\
\hline $\begin{array}{c}\text { Range of } \\
\text { Variation }\end{array}$ & $200 \mathrm{psi}$ & $180 \mathrm{psi}$ & $-22 \%$ \\
\hline Average & $541 \mathrm{psi}$ & $528 \mathrm{psi}$ & $-2 \%$ \\
\hline
\end{tabular}

Figure 10 shows the performance of the Anti-slug Advanced Control for another platform. After the implementation of the controller for all wells, the number of unscheduled shutdowns (trips) of the compressor has been decreased from an average of six to around 0.8 trips per month. As a consequence, gas flaring $\left(\mathrm{Nm}^{3} /\right.$ month) associated to compressor trip events were reduced substantially, as shown in figure 11. These results show that anti-slug advanced control has a positive impact on energy efficiency and the process sustainability, with emission reduction of 1200 ton $\mathrm{CO}_{2}$ per year by the platform. The economic gain, avoiding gas flaring was estimated around US $\$ 300.000,00$ per year for this unit.

Figures 10 and 11 also show that the advanced control was able to reduce significantly the variability of the number of shutdowns and the gas flaring, helping to increase safety and operational planning and predictability.

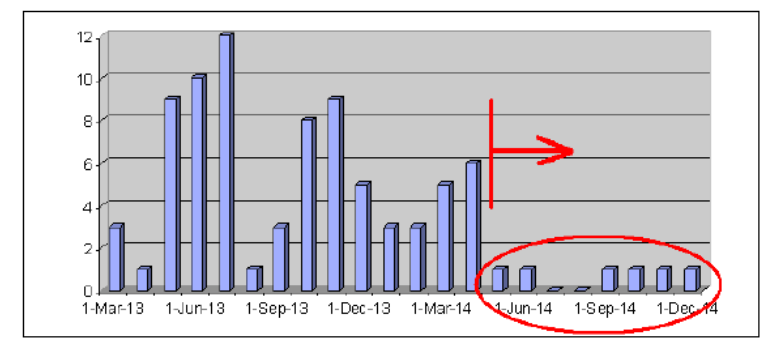

Fig. 10. Compressor trip reduction with anti-slug control.

A key point for success of any advanced control strategy is an effective participation of operators during design, commissioning and operation, in order to receive feedbacks which help to improve controller's performance. It was observed that during implementations described in this paper the involvement of operators were essential to change operational culture and for the success of the project. Figure 12 shows that the anti-slug advanced control has been used, in long term, more than $80 \%$ of the operating time, after commissioning. Operators only turn off the control during specific events as pigging pipelines.

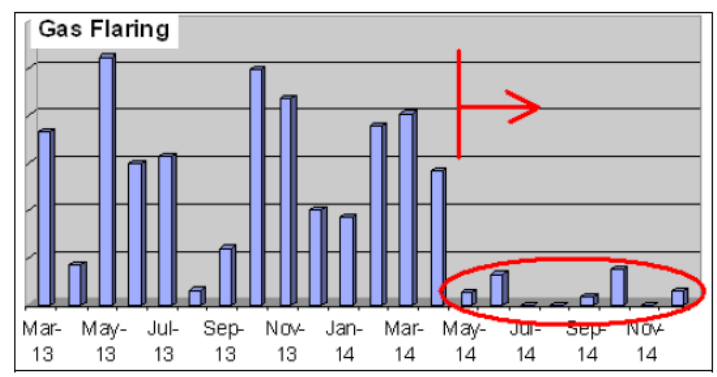

Fig. 11. Flaring reduction with less compressor shutdown.

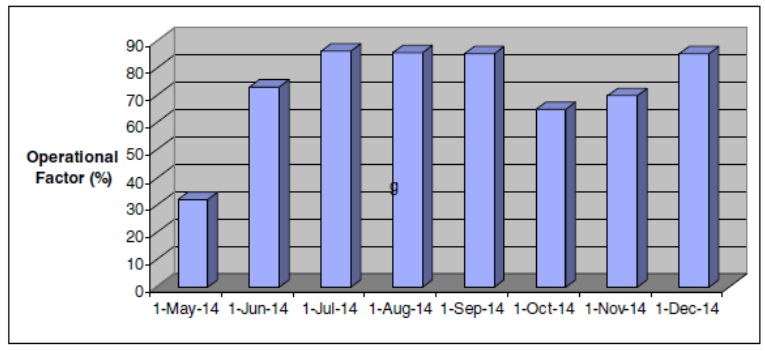

Fig. 12. Operational factor of the anti-slug control.

Operational efficiency is defined as the actual production of the month divided by the possible production considering the wells available and the plant conditions. Figure 13 shows that anti-slug control improved operational efficiency around $1.5 \%$ in one particular platform. Such improvement represents economic gains of approximately three millions of dollars per year for this particular offshore platform, considering the oil price of $60 \mathrm{US} \$$ / bbl. Although it is not trivial to establish a precise cause-effect relationship, we believe that this anti-slug advanced control was one of the major factors responsible for reducing production losses and increasing operational efficiency.

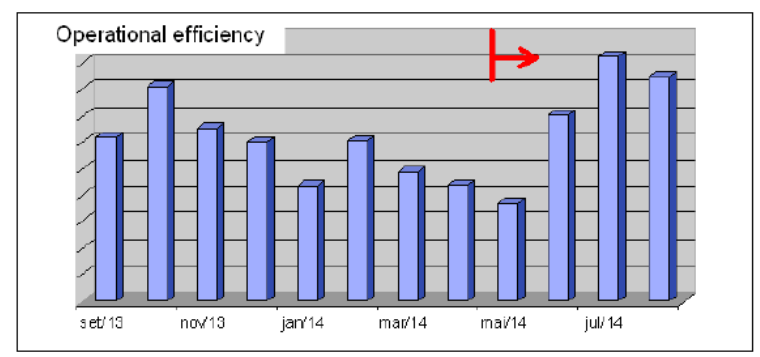

Fig. 13. Operational efficiency with the controller. 
A major challenge after implementation of anti-slug advanced controls is to keep applications in use and with good performance. Therefore, it is very important to keep continuous monitoring in order to tune parameters when necessary to adapt with changes in wells behaviors and process plants.

\section{CONCLUSION}

In this work, advanced anti-slug control strategies were described and results obtained for three offshore production units were discussed. It was observed that this controller was able to improve operational stability and safety, reducing events of high pressure in production header and the number of trips or unscheduled shutdown in compressor. As a consequence, flaring due to compressor trip was reduced substantially. The economic gains obtained by selling the spared gas were estimated around US\$ $300.000,00$ per year in one particular offshore platform. Another important gain of this advanced anti-slug control is to avoid flaring above the permitted by regulatory agencies avoiding fines payments, as a consequence.

In one platform, the system was able to increase operational efficiency in about $1.5 \%$, which represents an economic gain of about US\$ $3.000 .000,00$ per year. Although it is difficult to establish a precise cause-effect relationship, it was believed that this anti-slug advanced control was one of the major factors responsible for this better operational efficiency. Another qualitative gain with this advanced control system was to automate best operational practices, thereby implementing a kind of knowledge management, which ensures high productivity, even with operator's shift change or the arrival of young operators.

Finally, production platforms are becoming more complex and require advanced control systems to support operators and engineers in order to increase safety, profitability and operational reliability. This paper has presented the economic gains obtained with an anti-slug advanced control.

\section{ACKNOWLEDGEMENT}

We would like to thank all technicians and engineers from Petrobras, Trisolutions, Modec, PUC-RIO (Tecgraf), UFSC (Federal University of Santa Catarina), UFRJ (Federal University of Rio de Janeiro) and UFRGS (Federal University of Rio Grande do Sul) involved in development and implementation presented in the paper, as well as their help and contribution.

\section{REFERENCES}

American Petroleum Institute - API (2000). Guide to Advanced Control Systems. API Recommended Practice 557, First edition.

Campos, M., Teixeira, H., Liporace, F. and Gomes, M. (2009). Challenges and problems with advanced control and optimisation technologies. International Symposium on Advanced Control of Chemical Process - ADCHEM-2009..
Campos, M., Teixeira, A., Von Meien, O., Neto, S., and Santos, W. (2013). Advanced Control Systems for Offshore Production Platforms. Offshore Technology Conference, OTC Brazil.

Carvalho, M., Bomfim, C., Souza, D., Campos, M., Menezes, B. Caminhas, W., Alves, D. and Araujo, C. (2010). Desenvovimento do Controlador Não-Linear On-line Neuro-Fuzzy Controler - ONFC-DW e Aplicação na Indústria de Petróleo. XVIII Congresso Brasileiro de Automática (CBA) (in portuguese).

Carvalho, M. (2010). Controlador NeuroFuzzy com aprendizado on-line: Teoria e Aplicação na Indústria de Petróleo. Master thesis, Universidade Federal de Minas Gerais, Brazil, (in portuguese).

Dalsmo, M., Halvorsen, E. and Slupphaug, O. (2002). Active Feedback Control of Unstable Wells at the Brage Field. SPE Annual Technical Conference and Exhibition, (SPE 77650).

$\mathrm{Hu}$, B. (2004). Characterizing gás-lift instabilities, $\mathrm{PhD}$ Thesis, Norwegian University of Science and Technology.

Godhavn, J., Odhavn, J., Strand, S. and Skofteland, G. (2005a). Increased Oil Production by Advanced Control of Receiving Facilities, IFAC.

Godhavn, J., Fard, M. and Fuchs, P., (2005b). New slug control strategies, tuning rules and experimental results. Journal of Process Control, 15, 547-557.

Gouvea, M. R. (2005). Controle Neurofuzzy de Motor de Indução Com Estimação de Parâmetros e Fluxo de Estator. $\mathrm{PhD}$ Thesis, Universidade Federal de Minas Gerais, (in portuguese).

Kaasa, G., Alstad, V., Zhou, J. and Aamo, O. (2007).

Nonlinear Model-Based Control of Unstable Wells. Modeling, Identification and Control, 28, 3, 69-79.

Pickering, P., Hewitt, G., Watson, M. and Hale, C. (2001). The prediction of flows in production risers - truth \& myth? IIR Conference.

Sinegre, L. (2006). Dynamic study of unstable phenomena stepping in gas-lift activated wells. These Docteur de l'Ecole des Mines de Paris.

Stasiak, M., Pagano, D. and Plucenio, A. (2012). A New Discrete Slug-Flow Controller for Production Pipeline Risers, IFAC Workshop - Automatic Control in Offshore Oil and Gas Production.

Storkaas, E., Skogestad, S. and Godhavn, J. (2003). A Low Dimentional Model of Severe Slugging for Controller Design and Analysis. Proc. Multi Phase'03.

Storkaas, E. (2005). Stabilizing control and controllability. Control solutions to avoid slug flow in pipeline-riser systems. Ph.D. thesis, Norwegian University of Science and Technology.

Storkaas, E. and Skogestad, S. (2007). Controllability analysis of two-phase pipeline-riser systems at riser slugging conditions. Control Engineering Practice.

Taitel, Y. and Dukler, A. E. (1976). A model for predicting flow regime transitions in horizontal and near horizontal gas-liquid flow. AIChE Journal. 


\section{BIOGRAFIA}

No ano de 2008, se formou em Engenharia Elétrica na UNESP Universidade Estadual Paulista "Júlio de Mesquita Filho" - Campus de Ilha Solteira.

Em 2009, entrou para a Petrobras como Engenheiro de Petróleo. Realizou treinamento interno no CEP - Curso de Engenharia de Petróleo na Universidade Petrobrás em Salvador-BA. Em 2010, finalizou pós-graduação obtendo o título de especialista em Engenharia de Petróleo.

Ainda em 2010, se mudou para Macaé-RJ, onde trabalhou na operação onshore e offshore de plataformas de petróleo da Bacia de Campos.

Em 2011, se mudou para Santos-SP, onde exerce atualmente o cargo de Engenheiro de Petróleo na base de operações do FPSO Cidade de Santos que produz os campos de Uruguá e Tambaú, na Bacia de Santos. Neste período, participou de comissionamentos de poços de petróleo e gás natural, manifolds submarinos, unidades de injeção de água dessulfatada dentre outras atividades. Em suas atividades cotidianas elaborou e revisou procedimentos de partida e parada de poços, participou de estudos para otimização operacional, análises de risco e planejamento de paradas programadas. Pelas atividades desenvolvidas, tem contato com os desafios tecnológicos de onde surgem seus interesses acadêmicos. Como exemplos, tem-se a participação conjunta com a equipe da Petrobras em projetos de otimização de produção por controle de bandas de nível dos vasos separadores de plataformas, controle de golfadas dentre outros.

Seus interesses atuais são as ferramentas e métodos que permitam aumentos na eficiência de produção com redução de custos através dos menores tempos de parada operacional, menor consumo de diesel em poços e garantia de integridade de equipamentos, estando alinhado com as diretrizes estratégicas da companhia. 